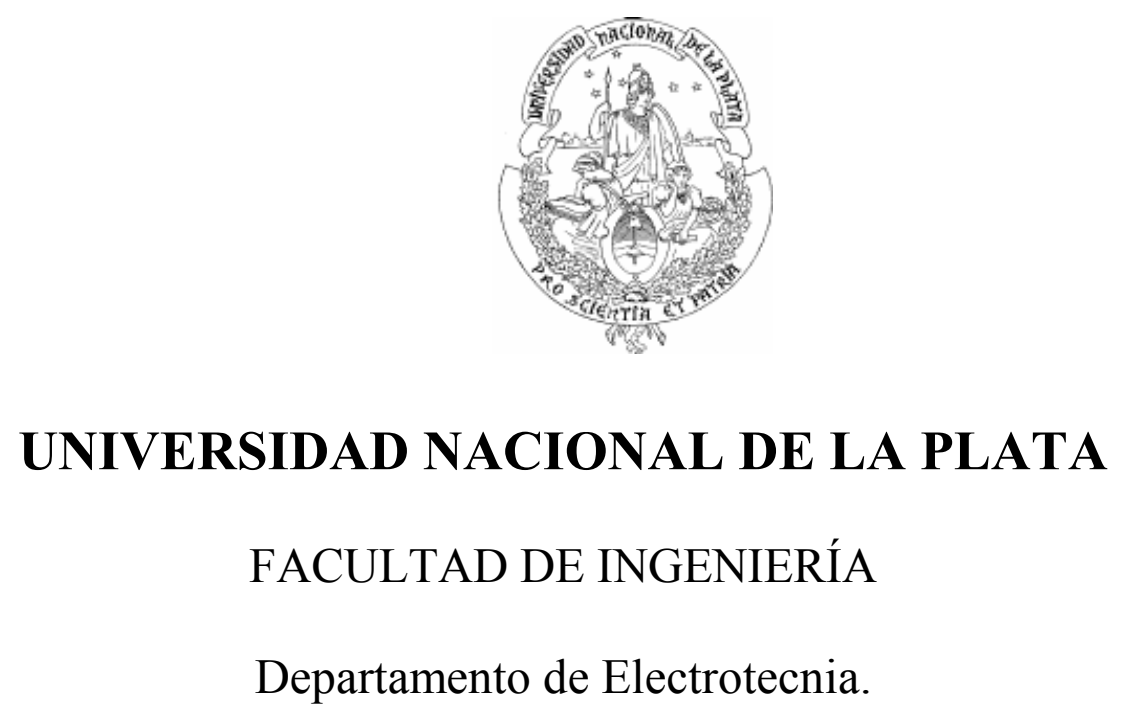

\title{
Amplificadores de Instrumentación en Aplicaciones Biomédicas.
}

\author{
Enrique Mario Spinelli \\ Tesis presentada para obtener el grado de \\ DOCTOR EN INGENIERÍA
}

Director: Dr. Miguel Angel Mayosky.

Codirector: Ing. Nolberto Martinez. 


\section{Resumen}

Durante décadas, el procesamiento analógico de señales fue el único tipo de procesamiento electrónico posible. Con el advenimiento de las técnicas digitales, se incorpora la posibilidad de procesar las señales digitalmente. A partir de la reducción del costo de los procesadores digitales de señales (DSP) y de los convertidores analógico-digital (ADC), el procesamiento analógico fue cediendo terreno al digital y se podría decir que perdió protagonismo en el escenario electrónico actual.

En aplicaciones biomédicas existen al menos dos tareas donde las técnicas analógicas son insustituibles: la amplificación de las débiles señales bioeléctricas a niveles apropiados para ser procesadas o digitalizadas y el rechazo a fuentes de interferencia electromagnética (EMI). Una señal comprometida en su rango de entrada por un gran nivel de EMI, no podrá ser mejorada aún con las más sofisticadas técnicas de procesamiento digital de señales. Además, una señal adquirida con EMI de $50 \mathrm{~Hz}$, resulta muy difícil de "limpiar" por técnicas digitales sin degradar su calidad; resultando más complejo aún realizarlo en tiempo real.

En esta tesis se analizan los mecanismos por los cuales la red de distribución de energía eléctrica $(50 \mathrm{~Hz})$ interfiere en el registro de biopotenciales. Se proponen aquí un modelo simple para describir estos mecanismos y métodos específicos para determinar sus parámetros en forma experimental.

Se analizan también las soluciones clásicas para la implementación de Amplificadores para Biopotenciales (A.B.) y se proponen circuitos originales apuntando a aplicaciones actuales como equipos alimentados a baterías con bajas tensiones de alimentación. Todo esto sin resignar características importantes en un A.B. como un elevado CMRR y una alta impedancia de entrada para señales de modo común.

Como un caso particular, se presenta el problema de adquisición de biopotenciales sin electrodo de masa ("sistemas de dos electrodos"); se analizan en detalle sus características en cuanto a su vulnerabilidad a EMI y se propone una estrategia para la implementación de A.B. específicos para este tipo de aplicación.

Los temas tratados en esta Tesis, tanto los de análisis como aquellos referidos a técnicas de diseño, fueron en primer lugar abordados en forma teórica, obteniendo expresiones analíticas útiles para la comprensión conceptual de los mecanismos involucrados y para el diseño de los circuitos. Luego, las expresiones obtenidas fueron validadas experimentalmente para lo cual, en algunos casos, se requirió el desarrollo de nuevas técnicas de medida. 


\section{ÍNDICE}

Capítulo 1: Introducción..................................................................................

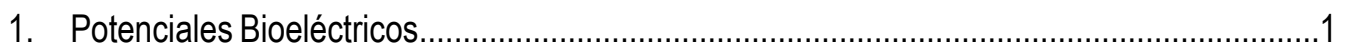

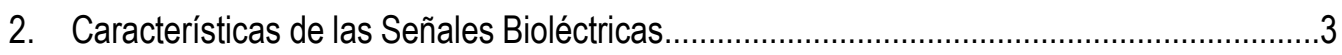

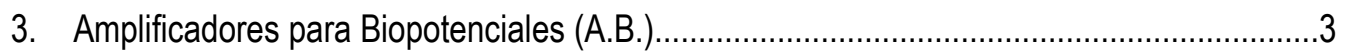

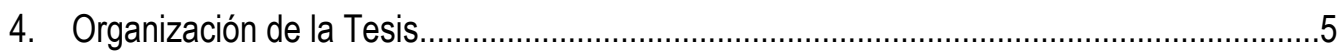

Capítulo 2: Sistemas de tres electrodos...........................................................

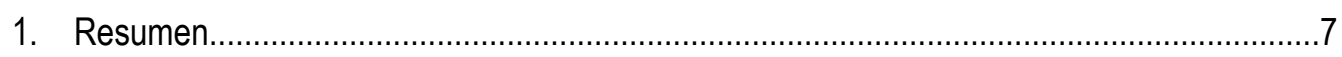

2. Modelo de Interferencia de la Tensión de Red..............................................................

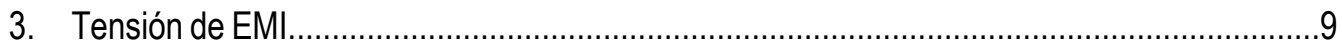

3.1. Mecanismos que producen tensión diferencial $\mathrm{V}_{\mathrm{iD.EMI}}$ en forma directa.....................11

3.1.1. Interferencia por Conducción Interna...........................................................11

3.1.2. Acoplamiento a los cables de conexión.........................................................13

3.1.3. Fem inducida en el "lazo de conexión" .......................................................15

3.2. Interferencia por Transformación de Modo............................................................17

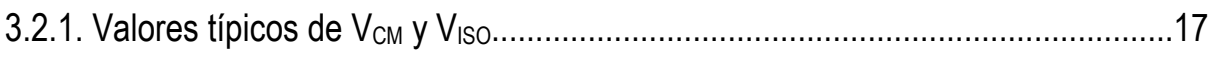

3.2.2. Transformación de M. Común / M. Diferencial Externa.....................................18

3.2.3. Transformación de Modo Común / Diferencial Interna (CMRR)........................19

3.2.4. Transformación de Modo Aislante / Modo Diferencial Externa...........................20

3.2.5. Transformación de Modo Aislante / Modo Diferencial Interna............................21

3.2.6. Interferencia debida al Transformador de Alimentación.....................................22

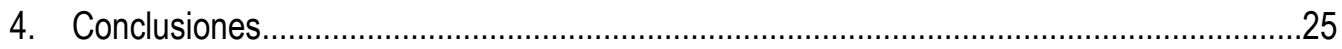


Capítulo 3: Sistemas de dos electrodos..........................................................27

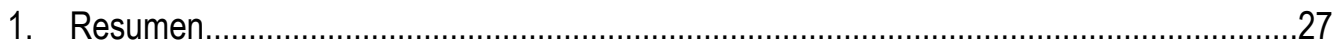

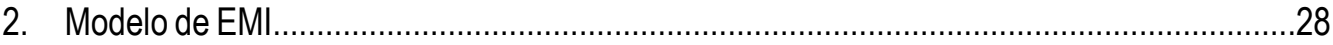

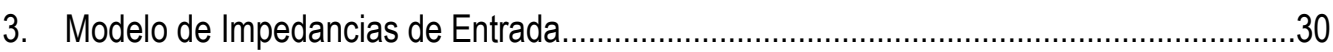

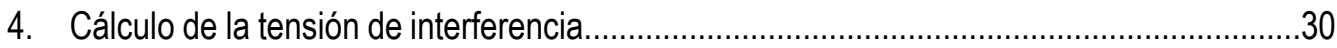

4.1. Mínima Impedancia de Acoplamiento Efectiva........................................................32

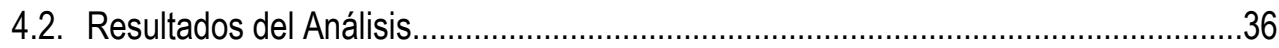

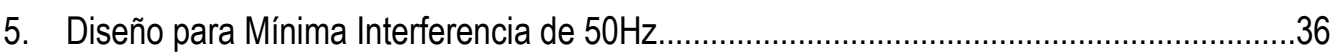

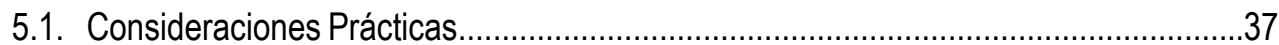

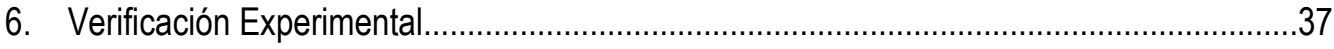

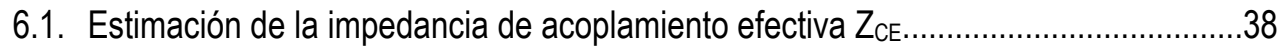

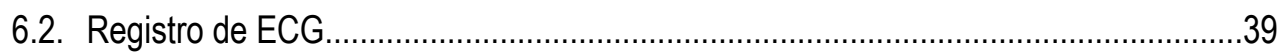

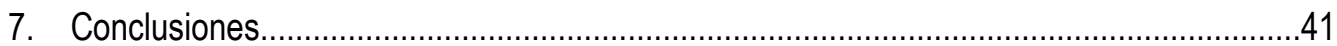




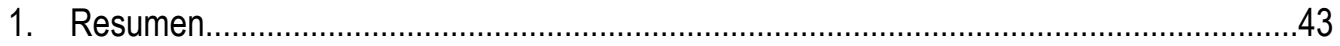

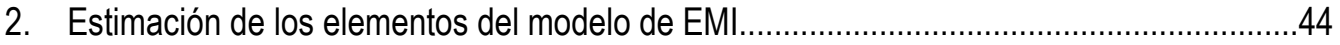

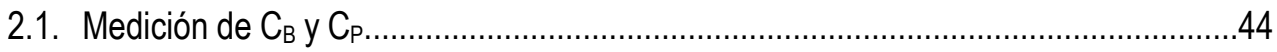

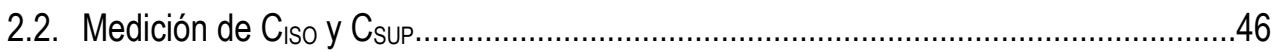

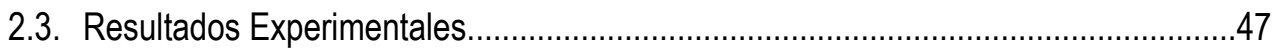

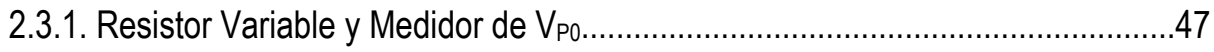

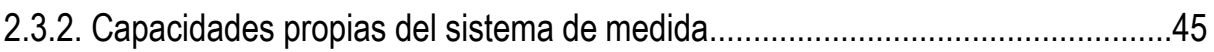

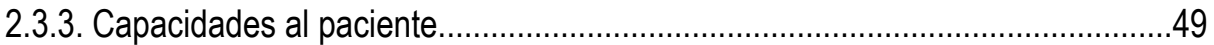

2.3.4. Capacidades propias del amplificador de biopotenciales..................................50

2.4. Medición de capacidades de acoplamiento a los cables de conexión........................51

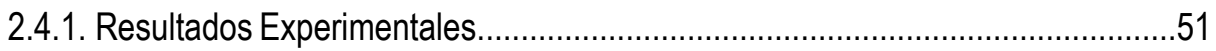

2.5. Medición de la capacidad propia de los cables de conexión......................................52

2.6. Medición del desbalance entre las impedancias de electrodo...................................52

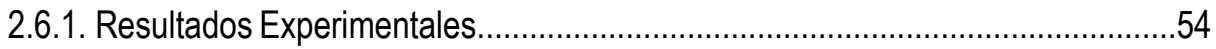

2.7. Medición de las Impedancias de Entrada de Modo Común.........................................57

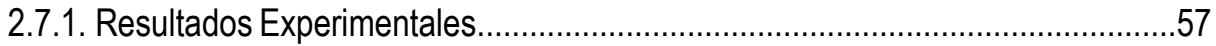

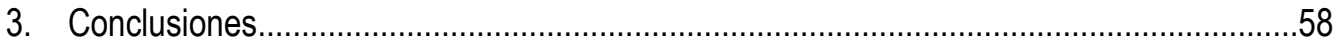


1. Resumen

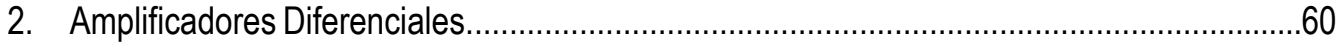

3. Configuraciones "bipolar" y "monopolar" en sistemas de tres electrodos..........................61

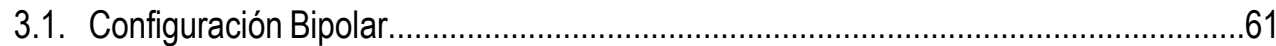

3.1.1. Amplificador Fully-Differential de 2 A.O........................................................

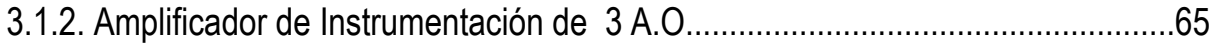

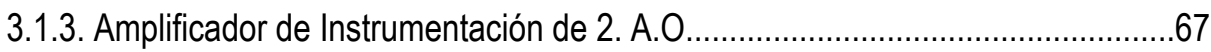

3.1.4. Amplificador de Instrumentación de 2 A.O. con ajuste de ganancia..................68

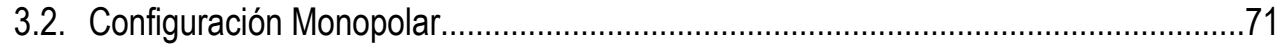

3.2.1. Un Amplificador Monopolar propuesto..........................................................72

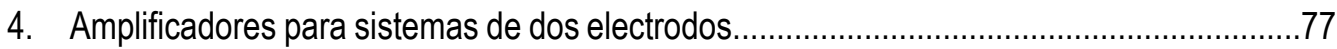

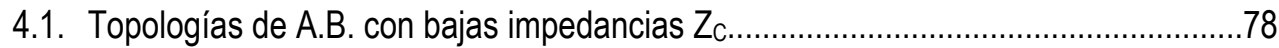

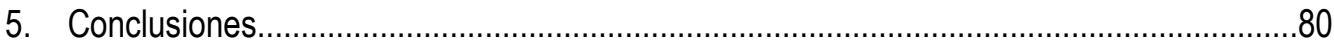


1. Resumen

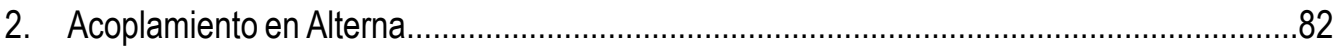

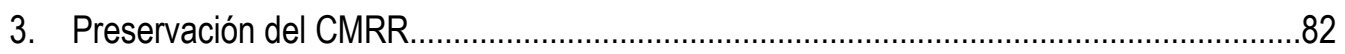

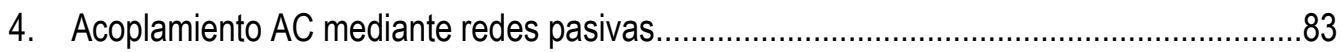

4.1. Resistores de polarización simples conectados a masa...........................................84

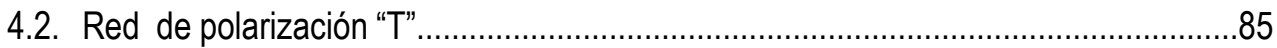

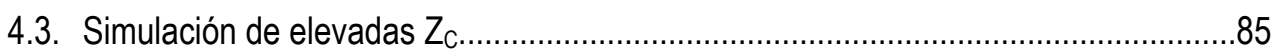

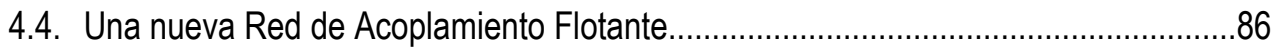

4.4.1. Componentes perfectamente apareados...................................................8

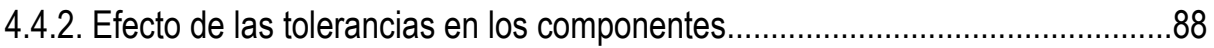

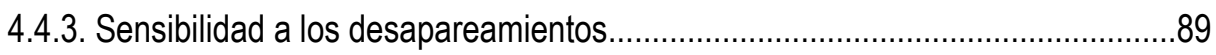

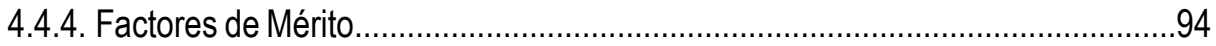

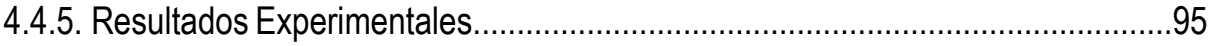

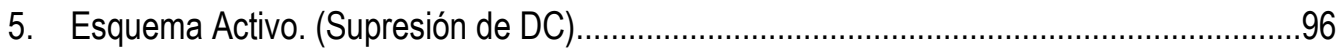

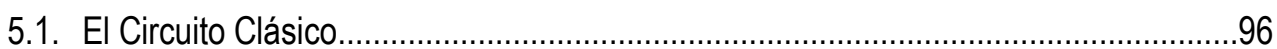

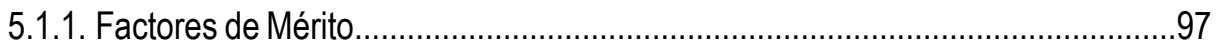

5.2. Una solución propuesta : Circuito Fully-Differential con supresión de DC..................99

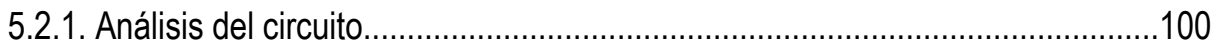

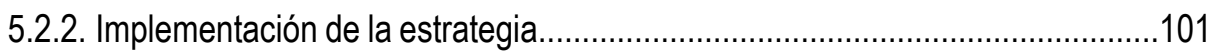

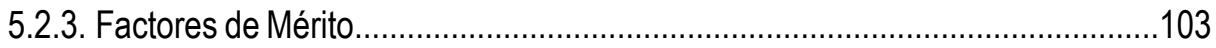

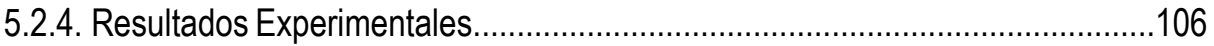

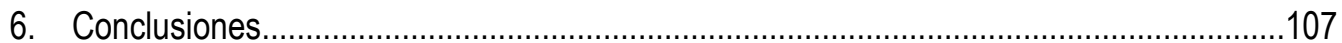


Capítulo 7: Mejora de las características de entrada de un A.B. mediante Realimentación de ModoComún......................................................109

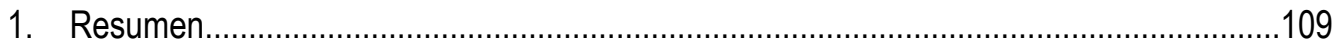

2. Reducción de la tensión de modo común. El circuito DRL............................................110

2.1. Estabilidad del Circuito DRL........................................................................111

2.2. Rechazo de la Tensión de línea.............................................................................115

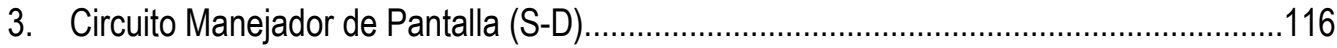

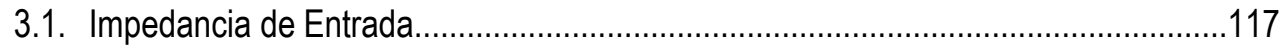

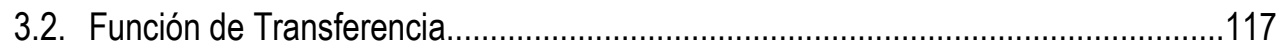

3.3. Resultados Experimentales..........................................................................120

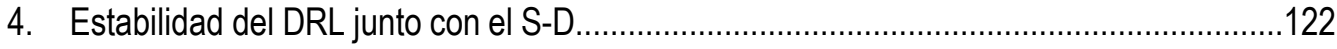

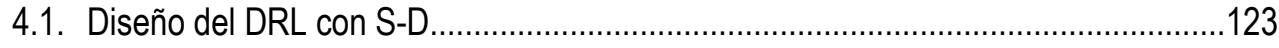

5. Control de $Z_{c}$ mediante realimentación de modo común..............................................124

5.1. Alta Zc: Amplificador con Bootstraping de Modo Común.......................................125

5.1.1. Estimación de la tensión de modo común....................................................126

5.1.2. Resultados Experimentales..................................................................127

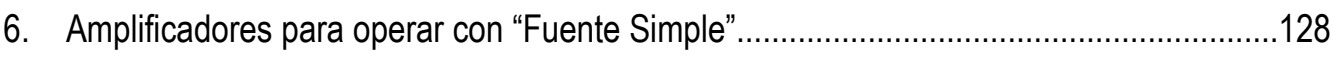

6.1. Acoplamiento en alterna + Tensión de polarización en DC.....................................129

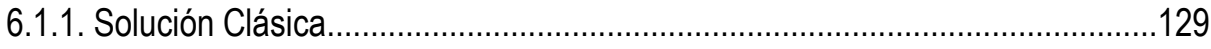

6.1.2. Una solución propuesta......................................................................129

6.2. Control de la tensión de Modo Común de Entrada................................................130

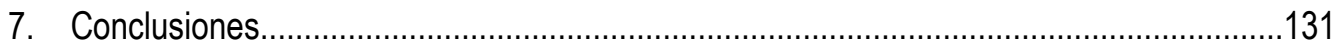




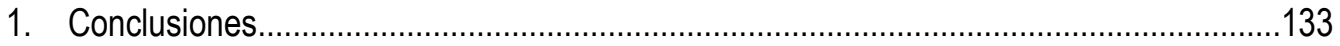

1.1. Análisis de EMI en Amplificadores de Biopotenciales...........................................133

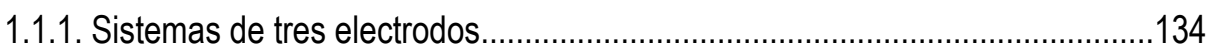

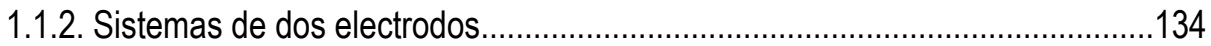

1.2. Diseño de Amplificadores para Biopotenciales....................................................135

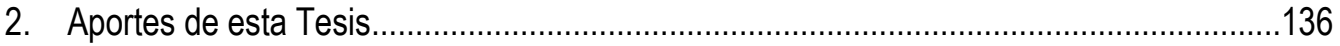

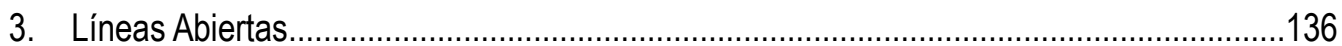

3.1. Sistemas de adquisición embebidos en prendas de vestir.....................................137

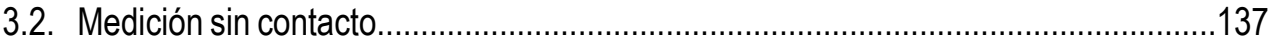

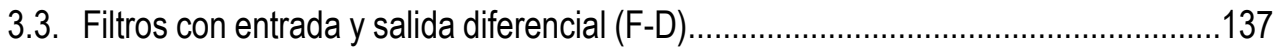




\section{Apéndices.}

Apéndice 1. Valores esperables de Tensiones de Modo Común y de Modo Aislante. 145

Apéndice 2. Análisis detallado del "Efecto Divisor de Potencial"...... 149

Apéndice 3. Cálculo de las tensiones de Modo Diferencial y de Modo Común en sistemas de 2 electrodos.

Apéndice 4. Simulación de resistencias de valor elevado 157

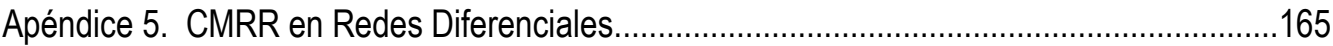

Apéndice 6. Cálculo del CMRR y ruido Propio para el I.A. F-D de dos A.O...........................167

Apéndice 7. Modelización de la Ganancia de Lazo Abierto y CMRR de un A.O......................171

Apéndice 8. Cálculo del Rechazo de Modo Común del Amplificador de Diferencia...............173

Apéndice 9. Cálculo de CMRR y Ruido Propio para el Amplificador de Instrumentación de dos A.O.

Apéndice 10. Caracterización de la Interferencia producida por equipos de Tubos Fluorescentes.

Apéndice 11. Sensibilidad de las raíces de un polinomio a variaciones en sus coeficientes

Apéndice 12. Tensión de Ruido debida a los Resistores de polarización. 191 


\title{
Algunas siglas utilizadas en esta Tesis.
}

\author{
A.B. : Amplificador para Biopotenciales. \\ A.O. : Amplificador Operacional. \\ AAMI : American Association of Medical Instruments. \\ AC : Corriente Alterna. \\ BW : Ancho de Banda. \\ CMRR : Rechazo de Modo Común \\ DC : Corriente Continua. \\ DRL : Driven-Right-Leg \\ ECG : electro-cardio-grama \\ EEG : electro-encéfalo-grama \\ EMG : electro-mio-grama. \\ EOG : electro-oculo-grama \\ F-D : Fully-Differential (Entrada Diferencial y Salida Diferencial) \\ F-E : Front-End \\ GPB : Producto Ganancia-Ancho de Banda. \\ S-D : Shield-Driver \\ $\mathrm{Z}_{\mathrm{C}} \quad$ : Impedancia de entrada de Modo Común. \\ $\mathrm{Z}_{\mathrm{D}} \quad$ : Impedancia de entrada de Modo Diferencial. \\ PP : Valor Pico-a-Pico de una señal. \\ RMS : Valor Eficaz de una señal (Root Mean Square). \\ PCB : Printed Circuit Board.
}


Siglas-Glosario 


\section{Capítulo 1: Introducción.}

\section{Potenciales Bioeléctricos.}

Durante más de un siglo, los potenciales de origen biológico denominados biopotenciales han demostrado ser una muy útil herramienta de diagnóstico médico.

Los métodos de diagnóstico basados en la interpretación de estos biopotenciales, por ejemplo, el electrocardiograma y el electroencefalograma, son utilizados en forma habitual en la práctica médica. Se trata de técnicas no invasivas, de bajo costo y de rápida interpretación por un profesional entrenado.

Los biopotenciales, se originan en la membrana externa de las células excitables, tales como las que componen el tejido nervioso o muscular. Estas señales eléctricas de origen iónico, se propagan a través de los tejidos circundantes que ofician de conductor de volumen, y pueden ser detectadas en la superficie del cuerpo mediante electrodos. Estas señales son de reducida amplitud y deben ser amplificadas para poder procesarlas o registrarlas.

En la interfase electrodo-piel-tejido ocurren complejos fenómenos electroquímicos (WEBSTER, 1992) que dan lugar a una transducción de corrientes iónicas a corrientes electrónicas, factibles de ser procesadas electrónicamente. Es decir, que en un sistema de adquisición de biopotenciales, los electrodos ofician de transductores. Las características de estos transductores son fuertemente variables, dependiendo de factores poco controlables como la preparación de la piel, del electrolito utilizado, la temperatura, la humedad, etc. Esto conduce a que el generador de señal accesible en los electrodos presente impedancias poco predecibles, imponiendo serias exigencias de diseño y en particular sobre la etapa de entrada o "front-end" (F-E). 
En la interfase electrodo-piel aparecen también semipilas de origen electroquímico que pueden llegar a tomar valores de hasta cientos de milivolts. Estos potenciales de continua (DC) pueden ser varios órdenes de magnitud mayores que la amplitud de las señales a detectar e imponen exigentes especificaciones en cuanto al rango de entrada del amplificador.

Una dificultad adicional en la adquisición de biopotenciales es la Interferencia Electromagnética (EMI) producida por fuentes externas, en particular por la tensión de la red de distribución eléctrica. Esta fuente de EMI ingresa al sistema de medida por diversos mecanismos, requiriendo del uso de técnicas específicas para su reducción y rechazo. La determinación precisa de la situación de EMI y su análisis constituyen un tema de investigación que se mantiene vigente (FERNÁNDEZ GIMENO, 2000).

Las características tan particulares del transductor y de la situación de EMI, hacen que los amplificadores de instrumentación para biopotenciales (A.B.) adopten características propias que los llevan a constituir una especialidad dentro de la instrumentación.

Día a día se incorporan especificaciones de diseño cada vez más estrictas, como reducción del consumo, menor número de componentes, alimentación a partir de baterías, rechazo de fuentes de EMI, etc. Estas exigencias crean un nuevo contexto en el cual los requerimientos resultan difíciles de cumplir utilizando soluciones "clásicas", invitando al aporte de ideas originales. En este último punto intenta contribuir esta tesis.

En el siglo pasado, para conseguir señales de buena calidad, era usual recurrir a técnicas invasivas como abrasión de la piel, electrodos subcutáneos o intracorticales. Estas soluciones apuntaban a mejorar la interfase electrodo-piel, mientras que la tendencia actual es trasladar la dificultad al diseño del sistema de medida.

Gracias al avance tecnológico incesante y la integración de ingenio a lo largo de los años, es posible obtener señales bioeléctricas reduciendo la invasividad, cumpliendo de este modo con uno de los principales objetivos de la tecnología: proporcionar confort.

El objetivo de esta tesis es desarrollar estrategias de diseño para amplificadores de biopotenciales que permitan adquirir señales de muy buena calidad, bajo condiciones adversas de medida y sin recurrir a técnicas invasivas. Se desea además, hallar soluciones que prioricen un bajo consumo, así también como un reducido número de componentes. Estas últimas características, especialmente importantes en el diseño de dispositivos portátiles. 


\section{Características de las Señales Bioléctricas}

Las señales bioeléctricas presentes a la entrada del A.B. son de reducida amplitud y sus anchos de banda (BW) dependen de cada aplicación. A solo modo de referencia, a continuación se indican algunos valores típicos para señales de uso común en diagnóstico médico como EOG: electro-oculograma, EEG: electro-encéfalograma, ECG: electro-cardio-grama y EMG: electro-mio-grama. En todos los casos se consideran señales captadas mediante electrodos superficiales.

\begin{tabular}{ccc} 
& Amplitud (typ) & BW (typ) \\
\hline EOG & $1 \mu \mathrm{V}$ & $10 \mathrm{~Hz}$ \\
\hline EEG & $10 \mu \mathrm{V}$ & $100 \mathrm{~Hz}$ \\
\hline ECG & $1 \mathrm{mV}$ & $100 \mathrm{~Hz}$ \\
\hline EMG & $100 \mu \mathrm{V}$ & $1 \mathrm{kHz}$ \\
\hline
\end{tabular}

Tabla 1. Amplitud típica y Ancho de Banda de algunas señales Bioléctricas. Los valores consignados son solo aproximados y a modo de referencia.

Los valores de la Tabla 1 son indicativos, pero permiten fijar los requerimientos de un A.B. La aplicación más exigente es el EEG: la amplitud de estas señales bioeléctricas es reducida, su BW es moderado pero la frecuencia de red $(50 \mathrm{~Hz})$ está dentro del ancho de banda de interés. Para conseguir una relación señal/ruido aceptable (de al menos 20dB), se requiere menos de $1 \mu \mathrm{V}$ de ruido en un ancho de banda de $100 \mathrm{~Hz}$. Suponiendo una densidad espectral de ruido constante, la misma debe ser menor a $10 \mathrm{nV} / \sqrt{\mathrm{Hz}}$. Es además deseable que la amplitud de la componente de $50 \mathrm{~Hz}$ (interferencia de la red) se mantenga por debajo del nivel de ruido, es decir menor a $1 \mu \mathrm{Vpp}$.

\section{Amplificadores para Biopotenciales (A.B.).}

El propósito de un A.B. es amplificar las débiles señales biológicas hasta obtener un nivel apropiado para que puedan ser registradas.

Un A.B. debe admitir altas tensiones de DC de modo diferencial a su entrada e importantes desbalances en las impedancias del generador de señal. Todo esto manteniendo buenas características; entre ellas, un alto rechazo de modo común (CMRR) y un bajo nivel de ruido. Estas condiciones son simples de cumplir asignando una ganancia importante a la etapa de entrada, pero esto se ve dificultado por las mencionadas componentes de DC. El problema fundamental de diseño de un A.B es disponer de una ganancia elevada en la primer etapa en presencia de componentes de DC.

En muchos casos, por ejemplo en estudios ambulatorios, o en la captura de biopotenciales mientras el paciente desarrolla su actividad normal (ej. holter), el A.B. suele operar en condiciones de EMI muy hostiles y su rechazo a los distintos tipos de interferencia adquiere especial importancia. 
La principal causa de EMI es la tensión de la red de distribución eléctrica. Los mecanismos por los cuales la misma ingresa al sistema de medida son conocidos (HUHTA \& WeBSTER, 1973) y día a día se desarrollan modelos cada vez más completos y precisos (PALlas ARENY, 1988) (METTING VAN RiJN., 1990) (CHIMENO FERNANDEZ, 2000). Esto permite estimar los niveles de EMI para distintas situaciones así también como desarrollar técnicas para reducir sus efectos.

La tensión de la red interfiere según diversas formas, una de ellas es a través de la tensión de modo común $V_{\mathrm{CM}}$. Esta tensión puede contaminar el registro debido a dos causas: el Rechazo de Modo Común CMRR finito del A.B. y a la transformación de modo común a modo diferencial debida al desbalance entre las impedancias de los electrodos. El primer inconveniente no es muy restrictivo (METTING VAN RIJN., 1990), pero el segundo exige, para el caso de mediciones con tres electrodos, elevadas impedancias de entrada de modo común $Z_{\mathrm{C}}$ (PALLAS ARENY, 1988), (WINTER, 1983a). Una forma de relajar en parte este requerimiento es utilizar circuitos activos, que a partir de una realimentación negativa de la tensión de modo común, permiten reducirla sustancialmente (WINTER, 1983b), (METTING VAN RIJN, 1990), (SPINELLI, 1999).

La topología históricamente más utilizada como primer etapa es el bien conocido amplificador de instrumentación (I.A.) de tres amplificadores operacionales (PALLAS ARENY, 1999). En general, esta etapa está acoplada en forma directa (DC) y las componentes de DC son eliminadas en etapas posteriores. Si se considera una tensión de offset de entrada de $\pm 300 \mathrm{mV}$ (AAMI, 1998) y un A.B. alimentado a partir de una fuente de alimentación simétrica de $+/-15 \mathrm{~V}$, es posible asignar a esta primer etapa una ganancia de hasta 50 veces $(\sim 30 \mathrm{~dB})$. Si bien usualmente se requieren etapas adicionales para completar la amplificación requerida, con esta ganancia el CMRR es importante (PALLAS ARENY, 1991), siendo además posible conseguir bajos niveles de ruido. Este último dependerá casi exclusivamente de los amplificadores operacionales (A.O) que componen la etapa de entrada.

Actualmente, existe una marcada tendencia a implementar equipos alimentados a baterías, tanto para uso portátil como en conusltorio (BURKE, 2000) (SPINELLI, 2000). Esta alternativa presenta elevadísimas impedancias de aislación, lo cual redunda en buenas características de rechazo a EMI y al mismo tiempo resulta muy seguro para el paciente. El hecho de alimentar el amplificador mediante baterías incorpora nuevas restricciones al diseño, apartándolo de las topologías clásicas. Tanto la tensión de alimentación como la energía disponible se tornan recursos escasos y una implementación con un reducido número de componentes se vuelve imperativa para reducir tamaño y a la vez consumo (METTING VAN RIJN, 1994).

En general, los amplificadores para biopotenciales utilizan tres electrodos: dos que proveen la señal biológica en forma diferencial y un tercero de referencia o masa. También es posible adquirir estas señales utilizando solo dos electrodos; una alternativa que resulta atractiva por reducir el número de electrodos instalados sobre el paciente, otorgándole mayor libertad de movimientos. 
En el caso de utilizar solo dos electrodos, el rechazo a EMI se vuelve fuertemente dependiente de la impedancia de entrada $Z_{C}$ que el amplificador presenta para señales de modo común. En 1980, Thakor y Webster, mostraron la factibilidad de esta técnica (THAKOR, 1980) apuntando a utilizar elevadas $Z_{\mathrm{C}}$ y posteriormente, Pallas Areny demostró que esta no es necesariamente la mejor elección (PALLAS ARENY, 1986). En los últimos años, si bien no se ha establecido cuales son las condiciones de medida más apropiadas, se han publicado circuitos que funcionan satisfactoriamente utilizando bajas $Z_{\mathrm{C}}$ (DOBREV, 2002.a), (DOBREV, 2002.b) dejando un interesante tema abierto por analizar.

\section{Organización de la Tesis.}

Esta tesis analiza el problema del diseño de Amplificadores para Biopotenciales y propone soluciones materializadas en circuitos concretos. En todos los casos, se intentó arribar a implementaciones adaptadas a los requerimientos actuales que apuntan a amplificadores con bajo consumo de energía y con un reducido número de componentes. La estructura adoptada para el desarrollo de estos temas es la siguiente:

A modo de introducción, en el CAPÍTULO 1, se presenta una descripción general del tema de tesis y de las condiciones de trabajo particulares de un A.B.

En el CAPítulo 2, se analiza en detalle el problema de la interferencia de la red de distribución en aquellos sistemas de adquisición de biopotenciales que utilizan tres electrodos (con electrodo de masa). El objetivo de este análisis es arribar a las condiciones óptimas de medida de las cuales se derivarán las características deseables en un A.B.

En el CAPÍtulo 3, se repite el análisis del Capítulo 2 para sistemas de dos electrodos. Se dedica un capítulo aparte a esta técnica porque sus condiciones de medida son muy particulares y distintas al caso de sistemas de tres electrodos.

En el CAPÍTULO 4, se proponen y desarrollan métodos experimentales para determinar los distintos parámetros de los modelos de interferencia presentados en los capítulos previos. Se incluyen aquí resultados experimentales que concuerdan aceptablemente con los predichos por el modelo de EMI.

En el CAPítulo 5, se analizan distintas topologías para la implementación de A.B. para sistemas de tres y de dos electrodos. Para cada caso, se determinaron sus características en cuanto a CMRR, impedancia de entrada de modo común $Z_{\mathrm{C}} \mathrm{y}$ niveles de ruido.

En el CAPÍtUlo 6 se presentan distintas técnicas para mejorar las características de la etapa de entrada de un A.B. Estas estrategias, basadas en la realimentación de la tensión de modo común, permiten mejorar notablemente las especificaciones del A.B. en particular su rechazo a la interferencia de la tensión de red y su impedancia de entrada de modo común. 
El CAPÍtulo 7, trata sobre el problema central del diseño de un A.B. que es el "acoplamiento en alterna", es decir cómo eliminar las componentes de DC presentes en su entrada a fin de poder asignar una ganancia importante a la primer etapa. Esto último redunda en altos CMRR y bajos niveles de ruido al mismo tiempo que conduce a una reducción del número de componentes necesarios. En este capítulo se presenta un análisis detallado de las distintas alternativas y se proponen soluciones basadas en redes de acoplamiento pasivas y esquemas activos que eliminan las componentes de DC mediante una adecuada realimentación negativa de las mismas.

En el CAPítulo 9, se exponen las conclusiones, se reseñan los aportes originales realizados y se esbozan las líneas futuras a seguir.

Con el objeto de facilitar la lectura, todos aquellos desarrollos extensos, que no son imprescindibles para la comprensión de los temas desarrollados, fueron incluidos en 12 Apéndices que componen la última sección de esta Tesis. 


\section{Capítulo 2: Sistemas de Tres Electrodos.}

\section{Resumen.}

La técnica más utilizada para adquirir biopotenciales emplea tres electrodos. Mediante dos de ellos se captura la señal bioeléctrica en modo diferencial y el restante opera como "electrodo de masa" o "electrodo de referencia".

En este capítulo, se analizan las características principales de los sistemas de tres electrodos, y en particular cómo estos son afectados por la interferencia producida por la tensión de red $(50 \mathrm{~Hz})$.

Si bien existen diversas soluciones dentro del procesamiento digital de señales, para eliminar la "contribución" de la tensión de red a posteriori, resulta difícil hacerlo sin degradar las señales (en particular su fase), y más difícil aun efectuar estos procesamientos en tiempo real.

Por otra parte, el nivel de interferencia puede comprometer el rango de entrada del amplificador, llegando a producir distorsión sobre la señal o directamente la saturación del A.B. Además, si la amplitud de la interferencia a la entrada del A.B. es significativa, puede producir, debido a la existencia de no-linealidades (tanto en la etapa de entrada como en las impedancias de electrodo), componentes de baja frecuencia dentro del ancho de banda de la señal de interés (VAN DER HORST, 1998)(DE JAGER, 1996).

Todo esto muestra la importancia de eliminar en forma "temprana" la interferencia de la tensión de red; es decir rechazarla antes que la misma ingrese al sistema, evitando así su propagación por las distintas etapas. Se analizan aquí los distintos mecanismos por los cuales interfiere la tensión de red y se proponen distintas técnicas para reducir sus efectos. De este análisis también se desprenden las características deseables de un A.B. para sistemas de tres electrodos. Las mismas establecen las principales especificaciones de diseño para estos amplificadores. 


\section{Modelo de Interferencia de la Tensión de Red.}

Como ya fue mencionado, la fuente de interferencia dominante en la adquisición de biopotenciales es la red de distribución de energía eléctrica $(50 \mathrm{~Hz})$. Dado que las dimensiones del sistema de medida son despreciables frente a la longitud de onda de esta fuente de EMI $\left(\lambda_{50 \mathrm{~Hz}} \simeq 6 \mathrm{~km}\right)$, se puede considerar que la situación corresponde a interferencia producida por campos cercanos (BENEDETTI, 2000), (OTT, 1988). Su influencia puede modelizarse mediante acoplamientos reactivos de tipo capacitivo (Campo Eléctrico) e inductivo (Campo Magnético).

En la Fig. 2.1 se muestra un esquema circuital que utiliza componentes concentrados para describir los principales mecanismos de acoplamiento en un sistema de medida de biopotenciales. Este esquema reúne modelos parciales utilizados en las principales publicaciones sobre este tema: (HUHTA, 1973), (METTING VAN RIJN, 1990), (MetTing VAN RiJn, 1991), (Fernández Chimeno, 2000), (WoOd., 1995) y (PALlas ARENY, 1989). Se considera aquí el caso de un sistema aislado ${ }^{1}$, es decir con una barrera de aislación definida entre el paciente y tierra.

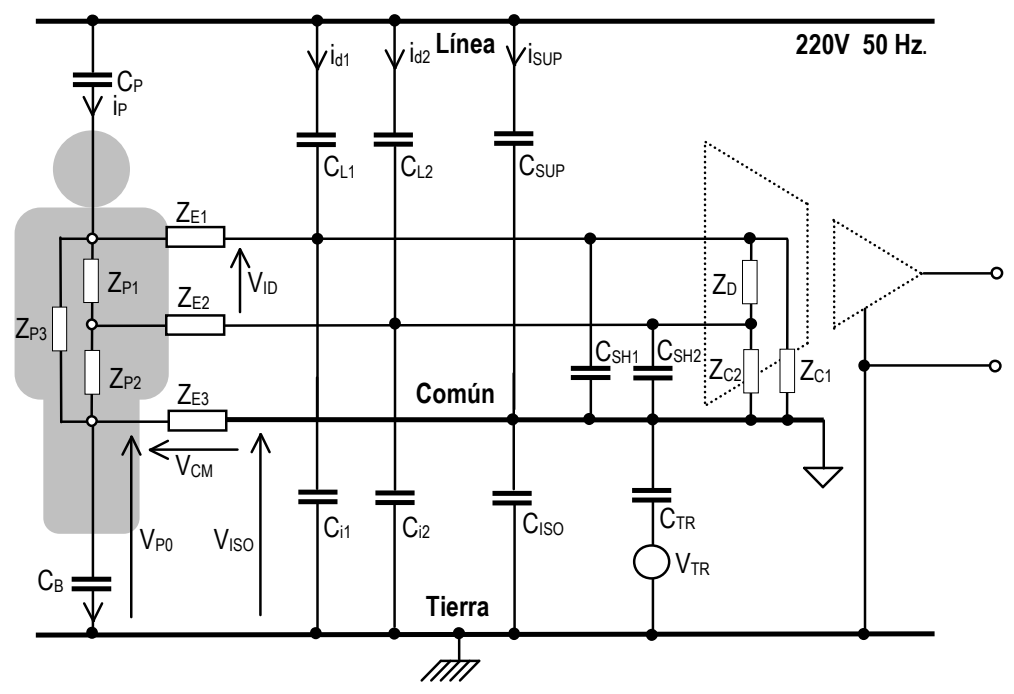

Figura. 2.1. Circuito que modela los distintos mecanismos de EMI en un Sistema de Adquisición de Biopotenciales.

En la Fig.2.1 se destacaron tres nodos que definen potenciales importantes para el análisis que sigue. Estos son: "Línea" que representa la tensión de la red de distribución, "Común" o "Masa", que es potencial a partir del cual se definen las señales propias del A.B. y "Tierra": el potencial de referencia general de todo el circuito.

Como puede observarse, el modelo contiene diversas impedancias. A continuación, se describen los elementos consignados en la Fig. 2.1 y se presentan valores típicos para algunos de ellos obtenidos de la literatura. En el CAPÍTULO 3 se presentarán métodos para determinar el valor de estos elementos en forma experimental.

\footnotetext{
${ }^{1}$ Actualmente este es el único tipo de sistema permitido.
} 


\section{Elementos del Modelo de EMI.}

$C_{\mathrm{P}} \quad$ : Capacidad Línea-Paciente. (0.2pF-5pF) (PALLAS ARENY, 1991)

$C_{\mathrm{B}} \quad$ : Capacidad Paciente-Tierra. (100pF-500pF) (PALLAS ARENY, 1991)

$C_{\mathrm{Li}} \quad:$ Capacidad Línea-Cable $i .(\approx 0.1 \mathrm{pF} / \mathrm{m}$ - Cable sin blindaje) (GRIMBERGEN, 1991)

$C_{\mathrm{i} 1}, C_{\mathrm{i} 2}:$ Capacidad Cable1-Tierra. $(\approx 10 \mathrm{pF} / \mathrm{m}$ utilizando Cable sin blindaje)

$C_{\text {SUP }} \quad$ : Capacidad Línea-Común. (0.1 pF- 100 pF) (MetTing VAn Risn, 1990)

$C_{\text {ISO }} \quad$ : Capacidad de Aislación Común-Tierra. (10-100 pF) (METting VAn Rijn., 1990)

$C_{\mathrm{SHi}} \quad$ : Capacidad Cable.i-Común ( $\approx 200 \mathrm{pF} / \mathrm{m}$ utilizando cable blindado)

$Z_{\mathrm{Ei}} \quad:$ Impedancia de contacto del electrodo $i$. (1k $\Omega-1 \mathrm{M} \Omega$ ) (ROSELL, 1988)

$Z_{\mathrm{Pi}} \quad$ : Impedancias internas del cuerpo. ( $\approx 500 \Omega$ ) (PALLAS ARENY, 1989)

$Z_{\mathrm{D}} \quad:$ Impedancia de entrada en modo diferencial.

$Z_{\mathrm{C}} \quad$ : Impedancia de entrada de modo común

$V_{\mathrm{P} 0} \quad$ : Tensión Paciente-Tierra.

$V_{\mathrm{CM}} \quad$ : Tensión de Modo Común.

$V_{\text {ISO }} \quad$ : Tensión de Modo Aislante.

$V_{\mathrm{TR}} \quad$ : Tensión de acoplamiento equivalente del transformador de alimentación.

$C_{\mathrm{TR}} \quad$ : Impedancia de aislación del transformador de alimentación.

\section{Tensión de EMI.}

La tensión de red imprime diferencias de potencial sobre las distintas impedancias expuestas en el modelo de la Fig.2.1, las cuales se presentarán al amplificador como tensiones de modo diferencial, de modo común y de modo aislante. Estas tensiones se definen como:

$V_{\mathrm{CM}}$ : Tensión de Modo Común. Diferencia de potencial entre el "paciente"2. y masa.

$V_{\text {ISO }}$ : Tensión de Modo Aislante. Diferencia de potencial sobre la barrera de aislación ${ }^{3}$.

$V_{\text {iD }}$ : Tensión de modo diferencial a la entrada del amplificador.

\footnotetext{
${ }^{2}$ Como muestra la Fig. 2.1, el "paciente" no tiene un único potencial sino que se definen varios nodos sobre él. Dado que las impedancias de conducción interna son bajas en comparación con las demás impedancias, es usual considerar al paciente isopotencial para tensiones de modo común. De este modo, la tensión $V_{\mathrm{CM}}$ queda de este modo definida sobre la impedancia del tercer electrodo $\left(Z_{\mathrm{E} 3}\right)$.

${ }^{3}$ Corresponde a la tensión común-tierra.
} 
Considerando una señal biológica nula, la tensión $V_{\text {oD.EMI }}$ presente a la salida del amplificador corresponde exclusivamente a la tensión de red (EMI) y está dada por:

Siendo,

$$
V_{\mathrm{oD.EMI}}=\left(V_{\mathrm{iD}}+\frac{V_{\mathrm{CM}}}{\mathrm{CMRR}}+\frac{V_{\mathrm{ISO}}}{\mathrm{IMRR}}\right) \cdot G_{\mathrm{DD}}
$$

$G_{\mathrm{DD}} \quad$ : Ganancia diferencial del A.B.

CMRR: Rechazo de Modo Común del A.B.

IMRR: Rechazo de Modo Aislante del A.B.

Por comodidad, esta tensión se refiere a la entrada definiendo $V_{\text {iD.EMI }}$ como:

$$
V_{\mathrm{iD.EMI}}=V_{\mathrm{iD}}+\frac{V_{\mathrm{CM}}}{\mathrm{CMRR}}+\frac{V_{\mathrm{ISO}}}{\mathrm{IMRR}}
$$

Los dos últimos términos de esta ecuación dependen de los rechazos de Modo Común CMRR y de Modo Aislante IMRR. En la forma que fue definida la ecuación (2.1), estas son propiedades intrínsecas del amplificador y representan su habilidad para rechazar, respectivamente, las tensiones de modo común y de modo aislante presentes en su entrada.

La tensión equivalente de EMI a la entrada $V_{\text {iD.EMI }}$, puede calcularse aplicando (2.2) luego de hallar $V_{\mathrm{CM}}$ y $V_{\text {ISO }}$ resolviendo el circuito de la Fig. 2.1. De esto resultará una expresión que involucrará la tensión de red, todas las impedancias indicadas en el circuito y los parámetros CMRR e IMRR propios del amplificador. Esta expresión resultaría tan compleja como inútil, ya que su complejidad mantendría ocultos los mecanismos por los cuales interfiere la tensión de red.

Para estimar $V_{\text {iD.EMI }}$, algunos autores proponen una versión simplificada del modelo de la Fig.2.1 que permite arribar a una solución analítica de complejidad aceptable (FERNÁNDEZ ChIMENo, 2000). Otra posibilidad extrema es seguir un camino puramente numérico, por ejemplo utilizando programas de simulación de circuitos (WoOD, 1995). Esto permite analizar completos modelos de interferencia y obtener resultados precisos ${ }^{4}$, pero no proporciona las expresiones analíticas adecuadas para efectuar un análisis conceptual.

En el análisis que sigue se optó por una técnica intermedia. Siguiendo un camino más conceptual que preciso, se estimará por separado la tensión debida a EMI que producen los principales mecanismos. Es importante notar que no es posible determinar la tensión total por simple suma de estas contribuciones (superposición), pero de todos modos este análisis proporcionará una clara idea de los órdenes de magnitud de los distintos aportes; permitirá analizar los distintos problemas presentes así también como proponer soluciones para minimizar cada uno ellos.

\footnotetext{
${ }^{4}$ La precisión de los resultados no es una cualidad importante en estudios de EMI. Los parámetros involucrados en este tipo de análisis, por ejemplo las impedancias asociadas a los acoplamientos, presentan incertidumbres tan grandes que no justifican una gran precisión en los cálculos.
} 
Como se mostró previamente, toda la tensión de salida del amplificador debida a EMI puede explicarse a partir de una tensión diferencial de entrada equivalente $V_{\text {iD.EMI }}$. Algunos mecanismos de interferencia producen genuinamente tensiones de modo diferencial, mientras que otros lo hacen indirectamente a partir de transformaciones de modo, es decir a través de tensiones de modo común o de modo aislante.

Dentro de los mecanismos que producen tensión diferencial en forma directa se analizarán: Interferencia por "conducción interna" dentro del paciente, por "acoplamiento a los cables de conexión" e interferencia producida por "fems Inducidas" en el lazo de conexión.

En cuanto a las posibles transformaciones de modo, se analizarán la de modo común a modo diferencial y la de modo aislante a modo diferencial. Estas transformaciones pueden ocurrir tanto fuera del A.B., esto es en la red de impedancias de la Fig.2.1 (transformación externa), como dentro del mismo (transformación interna) debido a su propio CMRR.

\subsection{Mecanismos que producen tensión diferencial $\mathrm{V}_{\mathrm{iD.EMI}}$ en forma directa.}

Son diversos los mecanismos por los cuales la tensión de línea puede producir tensiones de modo diferencial en forma directa. A continuación se analizan, en forma individual, los efectos más notables.

\subsubsection{Interferencia por Conducción Interna.}

Una fracción de la corriente $i_{\mathrm{P}}$ que ingresa al paciente circula sobre la impedancia de conducción interna del cuerpo $Z_{\mathrm{P} 1}$ (definida entre los electrodos $\mathrm{E}_{1} \mathrm{y} \mathrm{E}_{2}$ ), produciendo una diferencia de potencial de modo diferencial $V_{\mathrm{iD}}$. Para estimar la tensión producida por este efecto puede utilizarse el modelo reducido de la Fig. 2.2.

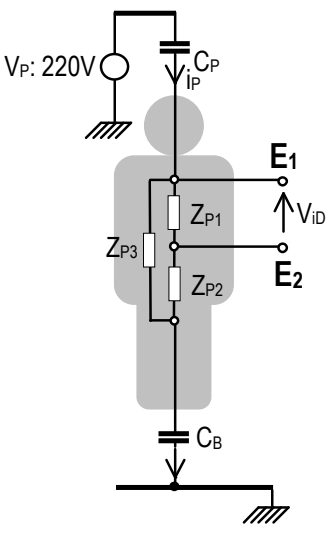

Figura 2.2. Interferencia por conducción interna sobre el paciente. La corriente İp, circulando sobre la impedancia de conducción interna $Z_{P 1}$ produce una tensión de modo diferencial $\mathrm{V}_{\mathrm{iD}}$. 
Considerando un caso desfavorable donde la totalidad de $i_{\mathrm{P}}$ circule sobre $Z_{\mathrm{P} 1}$, resulta:

$$
V_{\mathrm{iD}}=i_{\mathrm{P}} \cdot Z_{\mathrm{P} 1} \quad .
$$

En el peor caso, cuando toda la tensión de red queda aplicada sobre la capacidad $C_{\mathrm{P}}$ (paciente a potencial de tierra), la corriente $i_{\mathrm{P}}$ estará dada por:

$$
i_{\mathrm{P}} \approx 220 \mathrm{~V} \cdot \omega_{\mathrm{P}} \cdot C_{\mathrm{P}} \quad ; \quad \omega_{\mathrm{P}}=2 \pi 50 \mathrm{~Hz} .
$$

Combinando (2.3) y (2.4), se obtiene la tensión diferencial $V_{\mathrm{iD}}$ resultante:

$$
V_{\mathrm{iD}}=220 \mathrm{~V} \cdot \omega_{\mathrm{P}} \cdot C_{\mathrm{P}} \cdot Z_{\mathrm{P} 1}
$$

En (2.5) se observa que la tensión de EMI es proporcional a $Z_{\mathrm{P} 1}$. El valor de esta impedancia depende de la aplicación y en particular de la distancia que separa a los electrodos $E_{1}$ y $E_{2}$. Por ejemplo, en electrocardiografía (ECG), los valores esperables son del orden de los cientos de ohms (PALlAS ARENY, 1989).

Considerando una situación desfavorable: $Z_{\mathrm{P} 1}=500 \Omega$ y una capacidad $C_{\mathrm{P}}=5 \mathrm{pF}$, resultaría una tensión diferencial $V_{\mathrm{iD}}=170 \mu \mathrm{V}$.

En EEG (electro-encéfalo-grama), donde las señales son de reducida amplitud, los electrodos se encuentran más próximos entre si y $Z_{\mathrm{P} 1}$ es sensiblemente menor que en el ejemplo previo.

Por otra parte, la tensión $V_{\text {iD }}$ depende de la orientación de los electrodos respecto de la circulación de la corriente $i_{\mathrm{P}}$; si esta corriente fuera ortogonal, la tensión $V_{\mathrm{iD}}$ seria nula. De todos modos es una contribución a tener presente que puede ser dominante (SERRANO, 2003).

Finalmente se puede concluir que para este efecto, la tensión equivalente de EMI está dada aproximadamente ${ }^{5}$ por:

$$
\left.V_{\mathrm{iD.EMI}}\right|_{\text {Conduccion Interna }} \approx 220 \mathrm{~V} \cdot \omega_{\mathrm{P}} \cdot C_{\mathrm{P}} \cdot Z_{\mathrm{P} 1}
$$

Discusión.

Usualmente este mecanismo no produce niveles de interferencia importantes, pero tampoco hay mucho para hacer frente a este tipo de interferencia.

La situación es dependiente de la orientación del paciente y es posible que la interferencia disminuya cambiando su posición. Si los niveles de EMI debidos a este efecto no son aceptables, es necesario apantallar al paciente, por ejemplo colocando sobre él una "frazada conductora" conectada a tierra. De este modo, la corriente $i_{\mathrm{P}}$ será derivada a tierra y no atravesará $Z_{\mathrm{P} 1}$; pero como puede advertirse, no es una solución del todo práctica.

\footnotetext{
${ }^{5}$ La corriente $i_{\mathrm{P}}$ sobre $Z_{\mathrm{P} 1}$ y $Z_{\mathrm{P} 2}$ genera también tensión de modo común del mismo orden que $V_{\text {iD }}$, pero esta tensión es despreciable frente a la tensión de modo común normalmente presente sobre la impedancia del tercer electrodo. Igualmente despreciables serán sus efectos sobre $V_{\text {iD.EMI. }}$
} 


\subsubsection{Acoplamiento a los cables de conexión.}

Para analizar los efectos de las corrientes $i_{\mathrm{L} 1}, i_{\mathrm{L} 2}$ que ingresan a través de las capacidades de acoplamiento a los cables de conexión, resulta suficiente el circuito simplificado que se muestra en la Fig.2.3. Estas corrientes circularán sobre las impedancias de electrodos $Z_{\mathrm{E} 1}, Z_{\mathrm{E} 2}$ produciendo tensiones de modo diferencial.

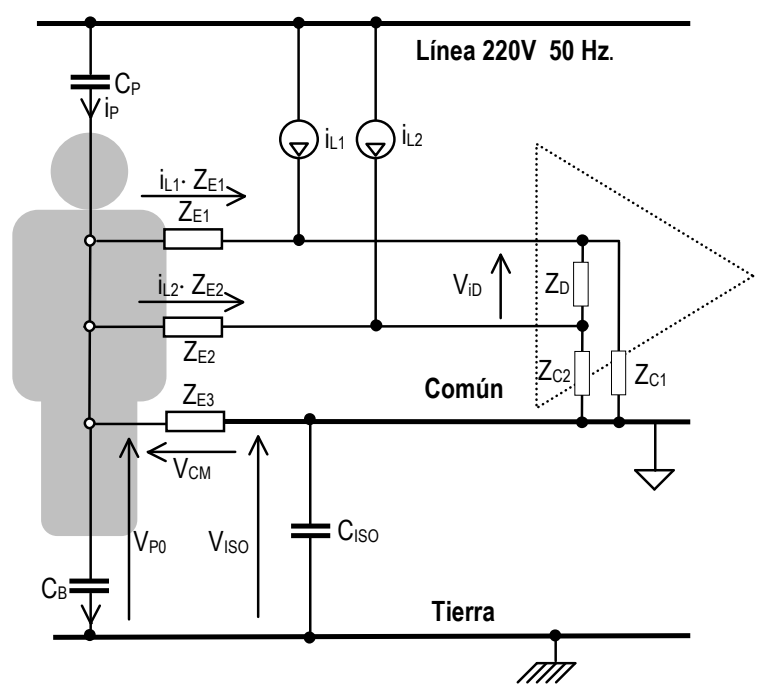

Figura 2.3. Interferencia por acoplamiento a los cables de conexión. Las corrientes $\mathrm{i}_{\mathrm{L} 1}, \mathrm{I}_{\mathrm{L} 2}$, circulan sobre las impedancias de electrodo $Z_{\mathrm{E} 1}$, $\mathrm{Z}_{\mathrm{E} 2}$ produciendo una tensión diferencial de entrada $\mathrm{V}_{\mathrm{D} D}$.

Considerando que las impedancias correspondientes a $C_{\mathrm{L} 1}$ y $C_{\mathrm{L} 2}$ (capacidades líneacable) son mucho mayores que $Z_{\mathrm{E} 1}, Z_{\mathrm{E} 2}$ y que también son elevadas frente a las impedancias asociadas a $C_{\mathrm{B}}$ y $C_{\mathrm{ISO}}$, las corrientes $i_{\mathrm{L} 1}$ e $i_{\mathrm{L} 2}$ pueden modelarse mediante generadores de corriente ${ }^{6}$. Estas corrientes, en el peor caso ${ }^{7}$, serán:

$$
\begin{aligned}
& i_{L 1}=220 \mathrm{~V} \cdot \omega_{\mathrm{P}} \cdot C_{\mathrm{L} 1} \\
& i_{L 2}=220 \mathrm{~V} \cdot \omega_{\mathrm{P}} \cdot C_{\mathrm{L} 2}
\end{aligned}
$$

Como las impedancias de entrada del amplificador son mucho mayores que las electródicas $Z_{\mathrm{E} 1}$ y $Z_{\mathrm{E} 2}$, las corrientes $i_{\mathrm{L} 1}, i_{\mathrm{L} 2}$ circularán a través de estas últimas produciendo una tensión diferencial de entrada dada por:

$$
V_{\mathrm{iD}}=i_{\mathrm{L} 1} \cdot Z_{\mathrm{E} 1}-i_{\mathrm{L} 2} \cdot Z_{\mathrm{E} 2}
$$

\footnotetext{
${ }^{6}$ Dicho de otro modo, las pequeñas corrientes inyectadas a través de estas capacidades no modifican sustancialmente los potenciales del circuito y por lo tanto las diferencias de potencial sobre $C_{\mathrm{L} 1}$ y $C_{\mathrm{L} 2}$ son constantes y también lo serán las corrientes que sobre ellas circulan.

${ }^{7}$ Esto significa que toda la tensión de línea queda aplicada sobre $C_{\mathrm{L} 1}$ y sobre $C_{\mathrm{L} 2}$, es decir considera el paciente a tierra. Esta situación actualmente no está permitida por las normas para Equipamiento Biomédico (AAMI, 1998).
} 
Normalmente las capacidades $C_{\mathrm{L} 1}$ y $C_{\mathrm{L} 2}$ son similares y (2.8) puede aproximarse por:

$$
\left.V_{\text {iD.EMI }}\right|_{\text {Ac. Cables de Conexion }} \approx 220 \mathrm{~V} \cdot \omega \cdot C_{\mathrm{L}} \cdot \Delta Z_{\mathrm{E}}
$$

siendo $\Delta Z_{\mathrm{E}}=Z_{\mathrm{E} 1}-Z_{\mathrm{E} 2}$ y $C_{\mathrm{L}}=0.5\left(C_{\mathrm{L} 1}+C_{\mathrm{L} 2}\right)$.

Esta tensión de interferencia puede ser muy significativa en caso de existir desbalances importantes en las impedancias electródicas. A modo de ejemplo, considerando valores típicos: capacidades $C_{\mathrm{L} 1}=C_{\mathrm{L} 2}=0.1 \mathrm{pF}$ e impedancias electródicas $Z_{\mathrm{E} 1}=10 \mathrm{k} \Omega, Z_{\mathrm{E} 2}=100 \mathrm{k} \Omega$. (una condición desfavorable pero no poco frecuente), resultan corrientes $i_{\mathrm{L} 1}, i_{\mathrm{L} 2}$ dadas por:

$$
i_{\mathrm{L} 1}=i_{\mathrm{L} 2}=220 \mathrm{~V} \cdot 2 \pi \cdot 50 \mathrm{~Hz} \cdot 0.1 \mathrm{pF}=6.9 \mathrm{nA},
$$

Estas corrientes producirán, de acuerdo a (2.9), una tensión de modo diferencial a la entrada de:

$$
V_{\mathrm{iD}}=6.9 \mathrm{nA}(100 \mathrm{k} \Omega-10 \mathrm{k} \Omega) \cong 0.6 \mathrm{mV} \quad .
$$

que es del orden de la amplitud esperable en la señal de ECG!!!

\section{Discusión.}

Este efecto produce una elevada tensión de EMI pero al mismo tiempo es muy simple de eliminar. Basta con conectar los electrodos al paciente mediante cables blindados, con sus blindajes conectados a masa. De este modo, como muestra la Fig. 2.4, se provee un camino de baja impedancia a las corrientes $i_{\mathrm{L} 1}, i_{\mathrm{L} 2}$, evitando que circulen a través de las impedancias de electrodo. Es importante notar que, debido a la capacidad entre el conductor central y la malla de los cables blindados, esta solución degrada la impedancia de entrada de modo común.

En ciertas aplicaciones, se requieren cables muy livianos y flexibles y no es posible (o muy caro) utilizar cables blindados. En estos casos, es importante utilizar cables con la menor longitud posible a fin de reducir $C_{\mathrm{L} 1}$ y $C_{\mathrm{L} 2}$. Otra medida a tomar para reducir estas capacidades, es evitar que los cables de conexión se apoyen sobre superficies de áreas importantes, es decir mantenerlos, en lo posible, "flotando en el aire" desde el electrodo hasta el amplificador. Por ejemplo, en estudios de EEG es común utilizar un pequeño tablero de conexiones próximo al paciente, el cual contiene los A.B. o al menos sus primeras etapas.

Una forma alternativa de reducir este tipo de interferencia es mantener las impedancias de electrodo $Z_{\mathrm{E} 1}$ y $Z_{\mathrm{E} 2}$ en valores reducidos. Esto último requiere necesariamente "preparar" la piel mediante métodos abrasivos, lo cual implica un grado de invasividad hacia el paciente que debería evitarse. Los equipos comerciales usualmente disponen de un sistema de medición de la impedancia de los electrodos para verificar que sus valores no sean demasiado elevados.

Existe otra solución para este problema que consiste en balancear las impedancias de electrodo $Z_{\mathrm{E} 1}, Z_{\mathrm{E} 2}$ insertando en serie con una de ellas una impedancia adicional que es ajustada en forma automática (YONCE, 2000). Esta alternativa es demasiado compleja para ser práctica, pero ciertas situaciones particulares pueden justificar su utilización. 


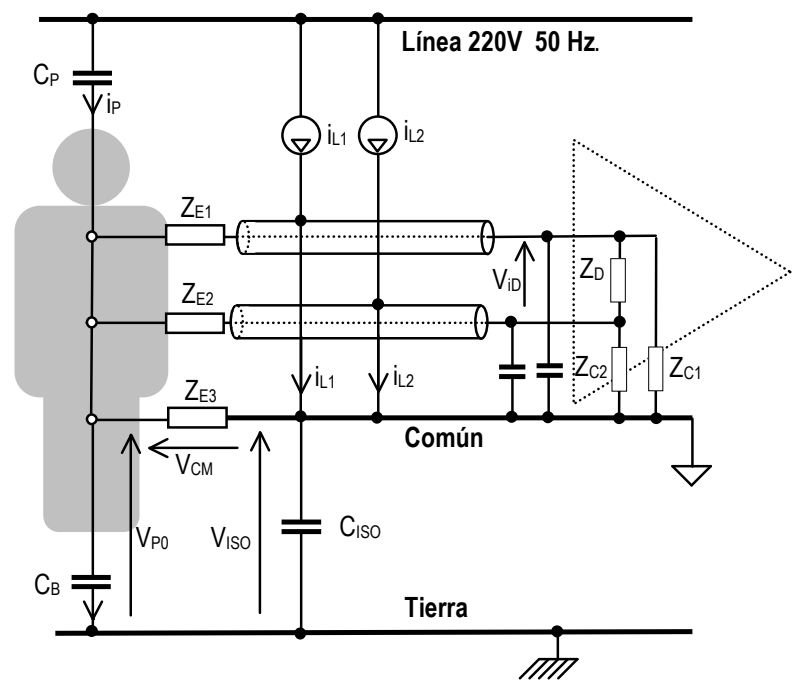

Figura 2.4. El efecto de la interferencia por acoplamiento a los cables de conexión puede evitarse fácilmente utilizando cables con blindaje. De este modo í1, i L2 $_{\text {s }}$ son derivadas a masa sin circular sobre las impedancias de los electrodos.

\subsubsection{Fem inducida en el "lazo de conexión".}

En la adquisición de biopotenciales, como en todo sistema de medida, existen diversos lazos cerrados (bucles) que definen áreas. Si un campo magnético variable incide sobre ellas, aparecerán fuerzas electromotrices inducidas. Requiere especial atención el bucle formado por los cables de conexión al paciente (ver Fig.2.5.a) porque las fems inducidas en este bucle se presentarán al amplificador como señales de modo diferencial y no podrán ser rechazadas.

Considerando una área expuesta $\mathrm{S}$ ortogonal a un campo magnético B sinusoidal, de pulsación $\omega_{\mathrm{p}}$, la fem inducida tendrá una amplitud dada por:

$$
\left.V_{\text {iD.EMI }}\right|_{\text {Campo Magnetico }}=B \cdot \omega_{\mathrm{P}} \cdot S
$$

La intensidad del campo magnético $B$, en una habitación no especialmente acondicionada es típicamente de 5nT a 300nT (PALLAS ARENY, 1989)(HUHTA, 1973). Considerando un área $S=0.2 \mathrm{~m}^{2}$ y un campo $B=300 \mathrm{nT}$, la tensión equivalente de EMI @ $50 \mathrm{~Hz}$ sería de unos $20 \mu \mathrm{V}$, la cual sólo sería significativa para aplicaciones muy baja amplitud (EEG ó EOG). 


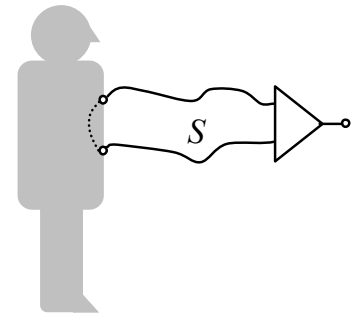

(a)

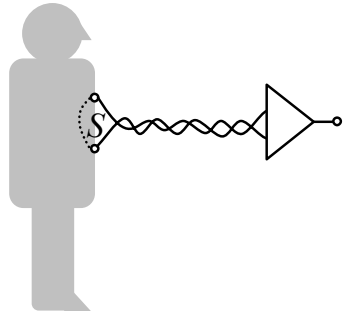

(b)

Figura 2.5. Interferencia por fems inducidas en el "lazo de conexión". (a) Las fems se inducen en el área $S$ expuesta al campo magnético. (b) Trenzando los cables puede reducirse el área expuesta y con esto la interferencia debida a este mecanismo.

Con una correcta disposición y trenzando de los cables de conexión, el área efectiva del lazo puede reducirse sensiblemente, pero restará siempre un área dada por el cuerpo del paciente (Fig.2.5.b). Existe una técnica denomina de lazo inverso (YАMАMOTO., 1998) que permite compensar esta área, la cual consiste en disponer un lazo de igual área al mencionado anteriormente, pero con polaridad inversa (Fig. 2.6). De este modo, si el campo magnético $B$ fuera uniforme en la zona que abarca a ambos lazos, las fems inducidas en cada uno de ellos serán de igual magnitud pero con signo opuesto y sus efectos se anularían. En la práctica, es difícil lograr un perfecto apareamiento entre las áreas, pero esta estrategia puede aportar mejoras de hasta $30 \mathrm{~dB}$ en el rechazo de EMI debida a campo magnético (YAMAMOTO, 1998).

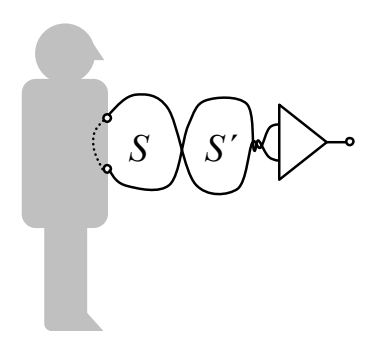

Figura 2.6. Método del Lazo inverso. Si las áreas $\mathrm{S}, \mathrm{S}^{\prime}$ son iguales, las fems inducidas en cada una de ellas se cancelan entre sí.

\section{Discusión.}

En aplicaciones críticas como podrían ser EEG o EOG, los electrodos se posicionan próximos entre sí. Las áreas expuestas son reducidas y la interferencia producida por fem inducida en el lazo de conexión no genera mayores inconvenientes.

La amplitud de estas tensiones es proporcional al área del lazo, por tal motivo es aconsejable reducirla en todo lo posible, por ejemplo trenzando los cables (Fig. 2.5.b). Esta es prácticamente la única medida a tomar para reducir este tipo de EMI (HUHTA, 1973). 
El blindado de los cables no aporta mejoras frente a este tipo de EMI de baja frecuencia. Solo resultaría efectivo un blindaje magnético de toda el área involucrada, pero no es una solución práctica para equipos portables. En aplicaciones donde la EMI por campo magnético constituye un problema serio se utilizan habitaciones especialmente acondicionadas con blindaje magnético (HAMALAIEN, 1993).

\subsection{Interferencia por Transformación de Modo.}

La tensión de línea produce importantes tensiones de modo común y de modo aislante. Dado que las señales biológicas son generalmente de modo diferencial, existe una separación de modo entre la señal y este tipo de interferencia. Esta separación puede aprovecharse utilizando un procesamiento selectivo al modo, por ejemplo un amplificador diferencial de alto CMRR. De esta forma es posible separar señal de EMI y rechazar la componente no deseada.

En un sistema de medida suelen existir transformaciones de modo que convierten, por ejemplo, señales de modo común en señales de modo diferencial "mezclando" señal y EMI. Luego, resultará imposible distinguir entre ellas, a menos que exista otro tipo de separación adicional, como por ejemplo espectral.

Las mencionadas trasformaciones de modo ocurren tanto dentro del amplificador como fuera de él, en la red externa de impedancias presentada en la Fig.2.1. Para obtener una idea del orden de magnitud de este problema, se estimarán en primer lugar los valores esperables para la tensión de modo común $V_{\mathrm{CM}} \mathrm{y}$ para la de modo aislante $V_{\text {ISO }}$. Posteriormente se calcularán los valores esperables para las tensiones de modo diferencial producidas como consecuencia de las mencionadas transformaciones de modo.

\subsubsection{Valores típicos de $V_{C M} y V_{I S O}$.}

La tensión de modo común $V_{\mathrm{CM}}$ está dada por la diferencia de potencial sobre la impedancia $Z_{\mathrm{E} 3}$ del electrodo de masa. Para estimar su valor en forma simple, se tendrá en cuenta que las impedancias de modo común $Z_{\mathrm{C} 1}, Z_{\mathrm{C} 2}$ y las asociadas a las pequeñas capacidades $C_{\mathrm{L} 1}, C_{\mathrm{L} 2}$ son mucho mayores que las restantes impedancias. El circuito simplificado, que considera al paciente isopotencial se muestra en la Fig.2.7.

Los valores que puede adoptar la tensión de modo común $V_{\mathrm{CM}}$ dependen de las capacidades $C_{\mathrm{P}}, C_{\mathrm{B}}, C_{\mathrm{SUP}}$ y $C_{\mathrm{ISO}}$ (ver APÉNDICE 1 ). Un par de casos representativos, asociados a valores extremos de $V_{\mathrm{CM}}$, pueden ser un amplificador dimensiones reducidas alimentado mediante baterías y un amplificador multicanal alimentado mediante un transformador. En el primer caso $V_{\mathrm{CM}}$ es de apenas unos $10 \mathrm{mV}$ mientras que para el segundo puede llegar a ser de hasta $500 \mathrm{mV}$ (APÉNDICE 1). Estos casos definen el rango de tensiones $V_{\mathrm{CM}}$ esperables:

$$
V_{\mathrm{CM}} \approx 10 \mathrm{mV}-500 \mathrm{mV}
$$

En cuanto a $V_{\text {ISO, }}$ (ver APÉNDICE 1), esta tensión puede adoptar valores de hasta 50V.

$$
V_{I S O}<50 \mathrm{~V}
$$




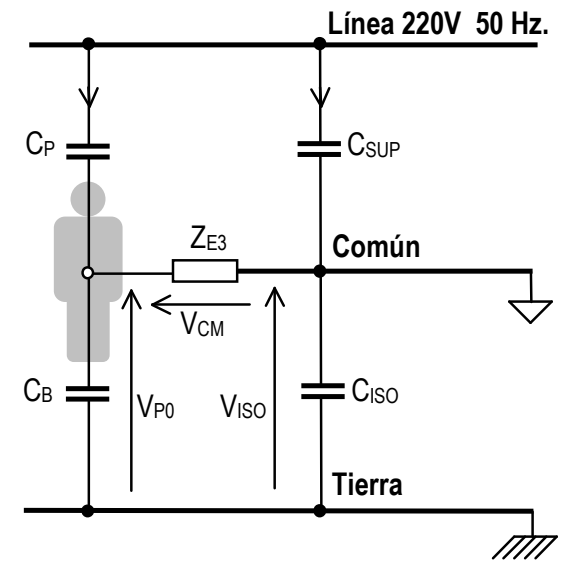

Figura 2.7. Modelo de EMI simplificado, útil para estimación de la tensión de modo común $\mathrm{V}_{\mathrm{CM}}$.

\subsubsection{Transformación de M. Común / M. Diferencial Externa}

La tensión $V_{\mathrm{CM}}$, debida a desbalances en las impedancias de electrodo, puede producir tensión diferencial. Esta transformación de modo, conocida como "efecto divisor de potencial" (HUHTA, 1973) puede explicarse fácilmente a partir del esquema simplificado de la Fig. 2.8.

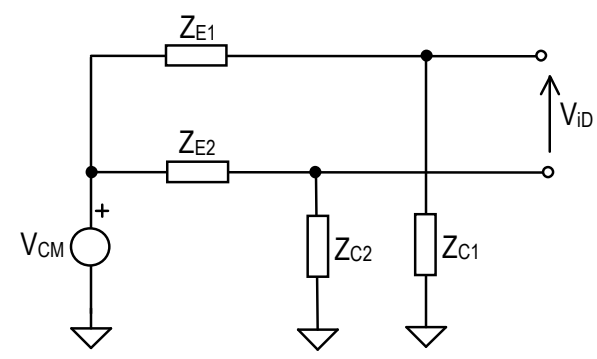

Figura 2.8. Efecto Divisor de Potencial. Las impedancias $Z_{E 1}, Z_{C 1}$ y $Z_{E 2}, Z_{C 2}$, componen respectivamente dos divisores de tensión. Si éstos no verifican la misma relación $Z_{\mathrm{Ci}} / Z_{\mathrm{Ei}}$, la tensión de modo común $\mathrm{V}_{\mathrm{CM}}$ producirá tensión de modo diferencial $\mathrm{V}_{\mathrm{iD}}$.

Como puede observarse, la tensión de modo común $V_{\mathrm{CM}}$ queda aplicada sobre dos divisores de tensión. Los mismos están formados por las impedancias de electrodo $\left(Z_{\mathrm{E} 1}, Z_{\mathrm{E} 2}\right)$ y por las impedancias de entrada que el A.B. presenta para tensiones de modo común $\left(Z_{\mathrm{C} 1}, Z_{\mathrm{C} 2}\right)$. Si la relación entre las impedancias que componen cada uno de estos divisores de tensión no son idénticamente iguales, la tensión $V_{\mathrm{CM}}$ producirá una tensión de modo diferencial $V_{\mathrm{iD}}$. Dado que las impedancias $\mathrm{Z}_{\mathrm{C}}$ son mucho mayores que $Z_{\mathrm{E}}$, la corriente sobre cada divisor es aproximadamente $V_{\mathrm{CM}} / Z_{\mathrm{C}}$ y tensión diferencial puede aproximarse por:

$$
V_{\mathrm{iD}} \cong Z_{\mathrm{E} 1} \cdot \frac{V_{\mathrm{CM}}}{Z_{\mathrm{C} 1}}-Z_{\mathrm{E} 2} \cdot \frac{V_{\mathrm{CM}}}{Z_{\mathrm{C} 2}}
$$


En general, la dispersión en $Z_{C}$ no es importante ${ }^{8}$ frente a las esperables en $Z_{\mathrm{E}} \mathrm{y}(2.15)$ puede escribirse como:

$$
\left.V_{\text {iD.EMI }}\right|_{\text {Transformacion de Modo Externa }} \cong V_{\mathrm{CM}} \frac{\Delta Z_{\mathrm{E}}}{Z_{\mathrm{C}}}
$$

siendo $\Delta Z_{\mathrm{E}}=Z_{\mathrm{E} 1}-Z_{\mathrm{E} 2}$.

Considerando $\Delta Z_{\mathrm{E}}=50 \mathrm{k} \Omega$ y $Z_{\mathrm{C}}=10 \mathrm{M} \Omega$, resulta una relación de transformación M.Común / M.Diferencial de $-46 \mathrm{~dB}$. En estas condiciones, una tensión $V_{\mathrm{CM}}=100 \mathrm{mV}$, produciría una tensión de modo diferencial $V_{\text {iD.EMI }}$ de $0.5 \mathrm{mV}$ !!!, que es del orden de la amplitud de una señal de ECG y un orden de magnitud mayor que una señal de EEG. Incrementando $Z_{\mathrm{C}}$ a $100 \mathrm{M} \Omega$ (un valor elevado pero posible), esta relación se reduce $\mathrm{a}-66 \mathrm{~dB}$.

\subsubsection{Transformación de Modo Común / Diferencial Interna (CMRR)}

Este tipo de transformación ocurre dentro del A.B. Debido a este efecto, una tensión de entrada de modo común, produce tensión de salida tal como lo haría una tensión de entrada de modo diferencial dada por:

$$
\left.V_{\text {iD.EMI }}\right|_{\text {Transformacion de Modo Interna }}=\frac{V_{\mathrm{CM}}}{\mathrm{CMRR}}
$$

Esta tensión normalmente es despreciable frente a la producida por trasformación de modo externa. Un amplificador modesto tiene CMRR mayores de $80 \mathrm{~dB}$, un valor muy superior a los $46 \mathrm{~dB}$ o $66 \mathrm{~dB}$ estimados en el punto previo para la transformación de modo externa.

\section{¿Cuánto CMRR es suficiente en un A.B.?}

Existe un histórico deseo por conseguir amplificadores con elevadísimo CMRR. Sin embargo, como se mostró anteriormente, la $V_{\text {iD.EMI }}$ debida al CMRR finito del amplificador es despreciable frente a la originada en la trasformación de modo externa.

Suponiendo un balance perfecto entre las impedancias de electrodo y una tensión de modo común elevada, por ejemplo de $100 \mathrm{mV}$, si se utilizara un A.B. con un CMRR de $80 \mathrm{~dB}$ resultaría una $V_{\text {iD.EMI }}=10 \mu \mathrm{V}$ : un valor aceptable por ejemplo para ECG. En una aplicación más sensible como EEG, se requieren unos $100 \mathrm{~dB}$ para que esta tensión se reduzca a $1 \mu \mathrm{V}$. En líneas generales se puede concluir que un CMRR de $100 \mathrm{~dB}$ es suficiente.

\footnotetext{
${ }^{8}$ En el APÉNDICE 2, se presenta una deducción más completa que incluye los desbalances en $Z_{\mathrm{C}}$.
} 


\section{Discusión.}

Existen varias medidas a tomar para reducir la interferencia producida por transformaciones de modo común a modo diferencial.

La impedancia de entrada de modo común $Z_{\mathrm{C}}$ debe aumentarse todo lo posible. Una $Z_{\mathrm{C}}$ de $100 \mathrm{M} \Omega$ es razonable considerando que usualmente los cables blindados presentan de capacidades de alrededor de $300 \mathrm{pF}$ (unos $100 \mathrm{M} \Omega$ a $50 \mathrm{~Hz}$ ). Para aumentar aún más $Z_{\mathrm{C}}$ se requiere utilizar circuitos Shield-Driver ${ }^{9}$ a fin de neutralizar estas capacidades.

La alternativa más conveniente es reducir $V_{C \mathrm{M}}$. Esto puede conseguirse con una buena aislación del A.B. es decir bajas $C_{\mathrm{P}}$ y bajas $C_{\mathrm{ISO}}$ (APÉNDICE 1) y/o utilizando circuitos activos reductores de Modo Común ${ }^{10}$. Esta última opción permite reducir $V_{\mathrm{CM}}$ en alrededor en $30 \mathrm{~dB}$ o más.

\subsubsection{Transformación de Modo Aislante / Modo Diferencial Externa.}

La Tensión de Modo Aislante $V_{\text {ISO }}$ también interfiere a través de transformaciones internas y externas.

Las capacidades entre los conductores de conexión y tierra, $C_{\mathrm{L} 1}, C_{\mathrm{L} 2}$, son pequeñas comparadas a las restantes capacidades que componen el modelo y su influencia en el circuito puede modelarse aceptablemente mediante generadores de corriente, resultando el esquema simplificado de la Fig.2.9.

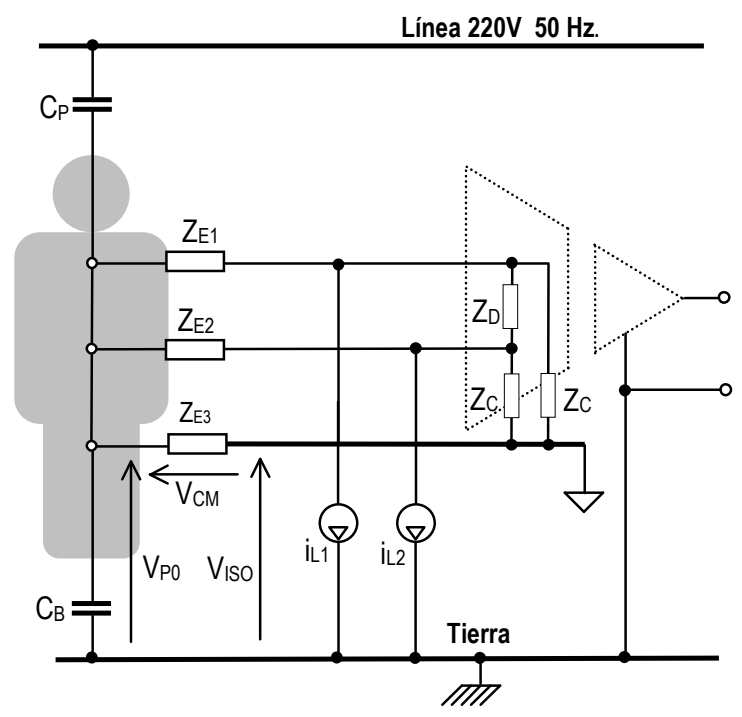

Figura 2.9. Modelo de EMI para la estimación de la transformación de Modo Aislante a Modo Diferencial producida en forma externa al A.B.

\footnotetext{
${ }^{9}$ Este tipo de circuito se analizará en el CAPÍTULO 7.

${ }^{10}$ Estos circuitos también serán analizados en el CAPÍTULO 7.
} 
Las corrientes de estos generadores están dadas por:

$$
\begin{aligned}
& i_{\mathrm{L} 1}=V_{\mathrm{ISO}} \cdot \omega_{\mathrm{P}} \cdot C_{\mathrm{L} 1} \\
& i_{\mathrm{L} 2}=V_{\mathrm{ISO}} \cdot \omega_{\mathrm{P}} \cdot C_{\mathrm{L} 2}
\end{aligned}
$$

y la tensión diferencial $V_{\mathrm{iD}}$ resulta:

$$
V_{\mathrm{iD}} \cong Z_{\mathrm{E} 1} \cdot V_{\mathrm{ISO}} \cdot \omega_{\mathrm{P}} C_{\mathrm{L} 1}-Z_{\mathrm{E} 2} \cdot V_{\mathrm{ISO}} \cdot \omega_{\mathrm{P}} C_{\mathrm{L} 2}
$$

Considerando $C_{\mathrm{L} 1}=C_{\mathrm{L} 2}=C_{\mathrm{L}}$, la (2.19) se reduce a:

$$
\left.V_{\mathrm{iD.EMI}}\right|_{\text {Transformacion de Modo Aislante Externa }} \cong V_{\mathrm{ISO}} \cdot \Delta Z_{\mathrm{E}} \cdot \omega_{\mathrm{P}} C_{\mathrm{L}} .
$$

Si bien las capacidades $C_{\mathrm{L} 1}$ y $C_{\mathrm{L} 2}$ son muy pequeñas, la tensión de modo aislante $V_{\mathrm{ISO}}$ suele ser muy elevada (del orden de la tensión de línea), resultando niveles de interferencia que pueden ser apreciables. Por ejemplo, tomando $V_{\mathrm{ISO}}=50 \mathrm{~V}$, cables sin blindaje con $C_{\mathrm{L} 1}=C_{\mathrm{L} 2}=0.1 \mathrm{pF}(30 \mathrm{G} \Omega @ 50 \mathrm{~Hz})$ y un desbalance $\Delta Z_{\mathrm{E}}=50 \mathrm{k} \Omega$, resulta una $V_{\text {iD.EMI }}$ de $80 \mu \mathrm{V}$, que no es aceptable para EEG.

Si se utilizan cables blindados, las corrientes $i_{11}, i_{12}$, indicadas en la Fig. 2.9, no circulan por las impedancias de los electrodos y sus efectos son despreciables. Quedan capacidades residuales entre las entradas del amplificador y tierra, que para reducir $V_{\text {iD.EMI }} 1 \mu \mathrm{V}$ (del orden del nivel de ruido típico de un A.B.) deben ser tan pequeñas como $0.01 \mathrm{pF}$.

\section{Discusión.}

La transformación de modo aislante a modo diferencial externa, no produce niveles de interferencia apreciables si se utilizan cables blindados. De todos modos, es importante reducir todo lo posible las capacidades entre las entradas del amplificador y tierra. Esto se consigue manteniendo la sección aislada (amplificador) tan separada de tierra como sea posible (METTING VAN RIJN, 1991), o mejor aún, utilizando un blindaje conectado a masa sobre el A.B.

Otra opción es reducir $V_{\text {ISO }}$, pero esto implica necesariamente reducir la impedancia de aislamiento y no luce muy recomendable. Metting Van Rijn, en (METTING VAN RIJN, 1991), proponen un circuito que realiza esta operación en forma controlada, limitando la mínima $Z_{\text {ISO }}$ a valores aceptados.

\subsubsection{Transformación de Modo Aislante / Modo Diferencial Interna.}

Si se requiere que la tensión de salida del A.B. sea referida a tierra (ver Fig.2.9), la señal debe atravesar la barrera de aislación común-tierra y puede ser afectada por la tensión $V_{\text {Iso }}$ presente sobre esta barrera. La tensión equivalente de entrada capaz de producir este efecto está dada por:

$$
\left.V_{\text {iD.EMI }}\right|_{\text {Transformacion de Modo Aislante Interna }}=\frac{V_{\text {ISO }}}{\text { IMRR }}
$$

Siendo IMRR el Rechazo de Modo Aislante del A.B. 
Actualmente, en general la barrera de aislación es atravesada en forma digital, por ejemplo mediante un dispositivo opto-acoplador y resulta prácticamente imposible que una tensión de modo aislante $V_{\text {ISo }}$ pueda alterar la señal transmitida. Esto implica un muy elevado IMRR por lo cual este tipo de interferencia no constituye un motivo de preocupación.

\subsubsection{Interferencia debida al Transformador de Alimentación.}

Como se mostró anteriormente, alimentar el A.B. mediante baterías es una opción muy conveniente, pues resultan bajas capacidades $C_{\mathrm{ISO}}$ y $C_{\text {SUP }}$ que conducen a muy reducidas tensiones de modo común $V_{\mathrm{CM}}$.

Cuando el consumo es elevado, por ejemplo en sistemas con un gran número de canales, el uso de un transformador de aislación es una alternativa para evitar el recambio o la carga frecuente de las baterías. El costo a pagar es un menor rechazo a la tensión de red.

El secundario de un transformador real no es perfectamente flotante, sino que existen impedancias que lo vinculan con la tensión de línea y con tierra. Estas impedancias pueden producir el acoplamiento de la tensión de línea a la señal de interés contribuyendo a $V_{\text {iD.EMI }}($ OTT, 1998). Un modelo simple de un transformador se muestra en la Fig.2.10.

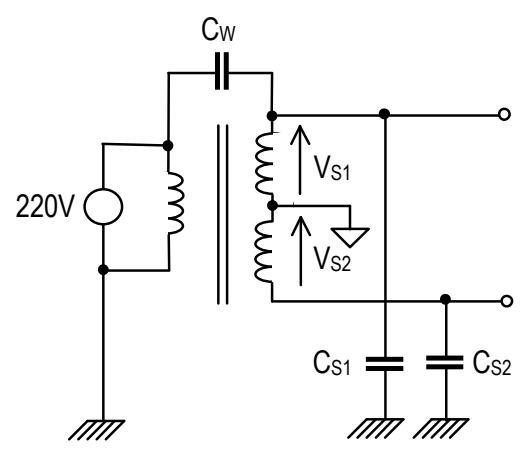

Figura 2.10. Circuito Equivalente del Transformador de alimentación, que considera sus capacidades parásitas.

Este modelo incluye las capacidades de acoplamiento $C_{\mathrm{W}}$ (primario-secundario) y las capacidades $C_{\mathrm{S} 1}, C_{\mathrm{S} 2}$ del secundario a tierra.

La capacidad $C_{\mathrm{W}}$ aumenta la capacidad entre línea y común (ver diagrama general de la Fig. 2.1), lo cual incrementa la tensión de modo común y resulta además inseguro para el paciente.

Los efectos de $C_{\mathrm{W}}$ pueden minimizarse en forma simple incluyendo un blindaje conectado a tierra sobre el primario (OTT, 1988), (MORRISON, 1998). De este modo, como muestra la Fig. 2.11, la corriente que circula por $C_{\mathrm{W}}$ es derivada a tierra a través del blindaje, preservando la seguridad del paciente y eliminando la vinculación líneacomún. 


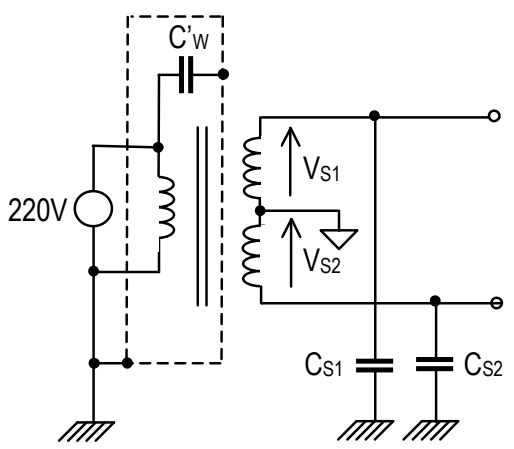

Figura 2.11. Circuito Equivalente de un Transformador de alimentación, que incorpora un blindaje sobre el bobinado primario.

Si bien el blindado del primario elimina la interferencia introducida por $C_{\mathrm{W}}$, el secundario del transformador puede también contribuir a generar tensión de EMI. Para explicar esto resulta útil obtener el modelo Thevenin del transformador visto entre tierra y masa. En la Fig.2.12.(a) se muestra el modelo del transformador y en la Fig.2.12.(b), su circuito Thevenin equivalente.

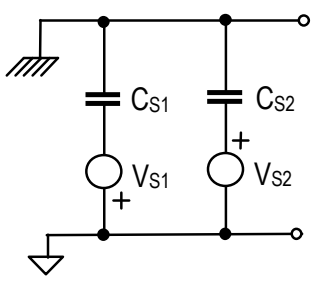

(a)

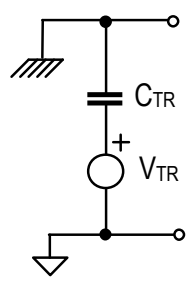

(b)

Figura 2.12. (a) Circuito Equivalente del transformador de la Fig. 2.11 y (b) su Equivalente Thevenin.

La tensión equivalente $V_{\mathrm{TR}}$ y la capacidad equivalente $C_{\mathrm{TR}}$ correspondientes a los elementos de la Fig. 2.12.(b), están dados por:

$$
\begin{aligned}
V_{\mathrm{TR}} & =V_{\mathrm{S}} \frac{\Delta C_{\mathrm{S}}}{2 C_{\mathrm{S}}}+\frac{\Delta V_{\mathrm{S}}}{2} \\
C_{\mathrm{TR}} & =2 \cdot C_{\mathrm{S}}
\end{aligned}
$$

siendo $V_{\mathrm{S}}=\left(V_{\mathrm{S} 1}+V_{\mathrm{S} 2}\right) / 2$ el promedio de las tensiones de secundario y $\Delta V_{\mathrm{S}}=V_{\mathrm{S} 1}-V_{\mathrm{S} 2}$ la diferencia entre ellas; $C_{\mathrm{S}}=\left(C_{\mathrm{S} 1}+C_{\mathrm{S} 2}\right) / 2$ es la capacidad promedio del secundario y $\Delta C_{\mathrm{S}}=C_{\mathrm{S} 1}-C_{\mathrm{S} 2}$ su diferencia.

En realidad, la capacidad $C_{\mathrm{TR}}$ forma parte de la capacidad de aislamiento $C_{\mathrm{ISO}}$. Se la mantiene aquí separada sólo para explicar el mecanismo de interferencia asociado al desbalance entre las tensiones del secundario del transformador. 
La tensión equivalente $V_{\mathrm{TR}}$ interfiere principalmente a través de la corriente que imprime sobre $Z_{\mathrm{E} 3}$, generando así tensión de modo común. Esta última contribuirá a $V_{\text {iD.EMI }}$ a través de una ${ }^{11}$ transformación de modo.

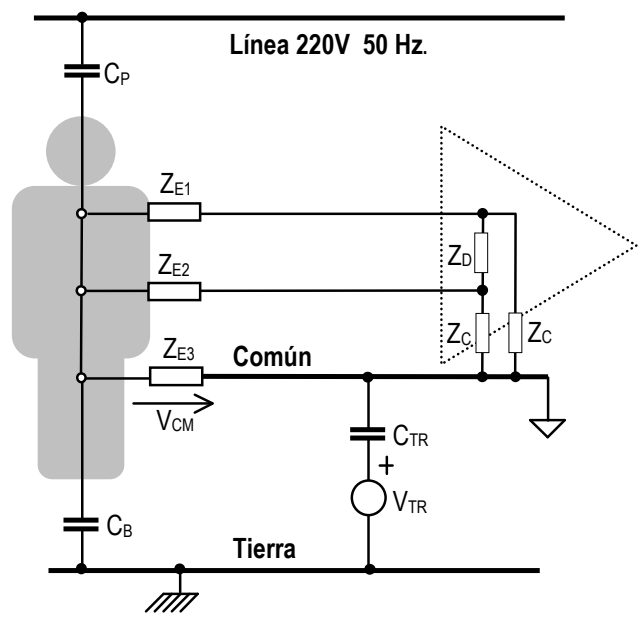

Figura.2.13. Interferencia por desbalances entre las tensiones del secundario del transformador de alimentación

La tensión de modo común $V_{\mathrm{CM}}$ debida a $V_{\mathrm{TR}}$, está dada aproximadamente ${ }^{12}$ por:

$$
V_{\mathrm{CM}} \cong \frac{V_{\mathrm{TR}}}{Z_{\mathrm{TR}}+Z_{\mathrm{CB}}} \cdot Z_{\mathrm{E} 3}
$$

La tensión de modo diferencial $V_{\text {iD.EMI }}$ que esta tensión $V_{\mathrm{CM}}$ puede producir depende de la trasformación de modo común / modo diferencial descripta en el punto 3.2.2. Combinando (2.23) con (2.16):

$$
\left.V_{\mathrm{iD.EMI}}\right|_{\text {Transformador de Alimentacion }} \cong \frac{V_{\mathrm{TR}}}{Z_{\mathrm{TR}}+Z_{\mathrm{CB}}} \cdot Z_{\mathrm{E} 3} \cdot \frac{\Delta Z_{\mathrm{E}}}{Z_{\mathrm{C}}}
$$

Tomando un caso desfavorable: un transformador de $V_{\mathrm{S}}=15 \mathrm{~V}$ con un desbalance en el secundario de $0.5 \mathrm{~V}$, una dispersión en $C_{\mathrm{S}}$ del $50 \%$ y $C_{\mathrm{S}}=150 \mathrm{pF}$ (el máximo valor admisible $\left.^{13}\right)$, los elementos del modelo Thevenin adoptan los siguientes valores:

$$
V_{\mathrm{TR}}=4.0 \mathrm{~V} ; Z_{\mathrm{TR}}=22 \mathrm{M} \Omega \text {. }
$$

\footnotetext{
${ }^{11}$ En realidad este efecto puede interpretarse como una doble transformación de modo: $V_{\mathrm{TR}}$ es una tensión de modo aislante, que debido a la $Z_{\mathrm{E} 3}$ no nula produce una tensión de modo común y finalmente ésta, debido al efecto divisor de potencial, se transforma en una tensión de modo diferencial.

${ }^{12}$ Esta expresión desprecia $Z_{\mathrm{E} 3}$ frente a la impedancia de $C_{\mathrm{B}}$ y de $C_{\mathrm{TR}}$.

${ }^{13}$ Con $C_{\mathrm{TR}}=150 \mathrm{pF}$, resulta una $Z_{\mathrm{TR}}$ de $22 \mathrm{M} \Omega$. En un sistema de $220 \mathrm{~V}$ esto produciría una corriente de pérdidas de $10 \mu \mathrm{A}$ que es la máxima admisible por la norma AAMI (AAMI, 1998).
} 
Considerando $C_{\mathrm{B}}=500 \mathrm{pF}$ y $Z_{\mathrm{E} 3}=100 \mathrm{k} \Omega$ resulta una tensión $V_{\mathrm{CM}}=14.5 \mathrm{mV}$. Esta tensión es del orden de la mínima $V_{\mathrm{CM}}$ esperable debida a la corriente $i_{\mathrm{P}}$ de acoplamiento al paciente (ver APÉNDICE 1). Sus efectos no son significativos.

\section{Discusión.}

La interferencia introducida por un transformador de alimentación, en general no es apreciable, pero igualmente es conveniente tomar algunos recaudos, como utilizar un transformador con punto medio, razonablemente balanceado ${ }^{14}$ y con una pantalla conectada a tierra.

En caso de utilizar un transformador sin punto medio, es decir con un único bobinado secundario, la tensión equivalente $V_{\mathrm{TR}}$ resulta elevada e igual a la tensión de secundario $V_{\mathrm{S}}$. Considerando la misma situación que en el ejemplo previo, resultaría una tensión $V_{\mathrm{CM}}=50 \mathrm{mV}$, que podría llegar a interferir apreciablemente.

\section{Conclusiones.}

Los mecanismos dominantes en cuanto a la interferencia producida por la tensión de red son el acoplamiento a los cables de conexión y el efecto "divisor de potencial". Para eliminar el primero basta con utilizar cables blindados, mientras que los efectos del segundo pueden minimizarse consiguiendo elevadas $Z_{C} y / o ́$ reduciendo la tensión de modo común.

La transmisión en forma digital a través de la barrera de aislación, es decir con el convertidor Analógico-Digital del lado del paciente, es una opción muy conveniente, pues proporciona un muy elevado rechazo IMRR a tensiones de modo aislante. Para preservar el IMRR también es importante mantener una baja capacidad entre las entradas del A.B. y tierra y/o blindar estas últimas a potencial de masa.

El CMRR de un A.B. no es un requerimiento crítico. En muchos casos, un CMRR de 80 o $90 \mathrm{~dB}$ es suficiente y un CMRR de $100 \mathrm{~dB}$ cubre todas las aplicaciones biomédicas. Este último valor es elevado, requiere un cuidadoso diseño del A.B., pero no es difícil de conseguir.

En cuanto a aquellos sistemas que utilizan transformador de alimentación, si el mismo está razonablemente balanceado, sus efectos no son significativos más allá del incremento en las capacidades masa-línea $C_{\mathrm{P}}$ y masa-tierra $C_{\mathrm{ISO}}$.

En conclusión, para mediciones de biopotenciales que utilicen tres electrodos (con electrodo de masa), un A.B. debe tener un CMRR elevado y una $Z_{\mathrm{C}}$ tan alta como sea posible. Resulta además muy conveniente reducir la tensión de modo común $V_{\mathrm{CM}}$. Estas son las principales consideraciones de diseño para un sistema de adquisición de biopotenciales.

${ }^{14}$ Esto puede conseguirse fácilmente bobinando los dos secundarios juntos utilizando el alambre doble. 
Capítulo 2 - Sistemas de tres electrodos 


\section{Capítulo 3. Sistemas de Dos Electrodos}

\section{Resumen}

El registro de biopotenciales puede realizarse utilizando solo dos electrodos, es decir sin electrodo de referencia o masa. Esta técnica es atractiva por varias razones: la preparación del paciente es más simple, otorga mayor libertad de movimientos y representa un menor costo en electrodos. Por otro lado, las mediciones de "dos electrodos" son muy vulnerables a la interferencia de la tensión de red (WOOD, 1995), requiriendo un cuidadoso diseño del amplificador y de sus circuitos accesorios.

Del mismo modo que en los sistemas de tres electrodos, la señal bioeléctrica a registrar es la diferencia de potencial entre los "electrodos activos", pero en este caso, al no existir electrodo de masa, la tensión de modo común puede adquirir valores elevados. Las condiciones de EMI para estos sistemas, difieren significativamente del análisis presentado en el CAPÍTULO 2 para mediciones que utilizan electrodo de masa y por esta razón se le dedica este capítulo especialmente.

Una particularidad de esta técnica es la dependencia de su rechazo a la tensión de red con la impedancia de entrada del amplificador para tensiones de modo común $Z_{\mathrm{C}}$. A diferencia del caso clásico de "tres electrodos", un elevado valor de $Z_{\mathrm{C}}$ no es siempre la mejor opción.

En este capítulo se presenta un modelo que describe la influencia de la tensión de red para distintos valores de $Z_{\mathrm{C}}$. Del análisis de este modelo resulta que, dada una situación de EMI definida por las capacidades de acoplamiento, la condición de mínima interferencia se produce para valores extremos de la impedancia de entrada de modo común, esto es una $Z_{\mathrm{C}}$ nula o infinita. Como se demostrará aquí, la conveniencia de uno u otro caso, depende del CMRR del amplificador y de las capacidades asociadas al modelo de EMI analizado en el CAPÍTULO 2. 


\section{Modelo de EMI.}

Como se mostró en el CAPÍTULO 2, son diversos los caminos por los cuales la tensión de red ingresa a un sistema de adquisición de biopotenciales. Tomando algunos cuidados, por ejemplo utilizando cables trenzados y blindados, el aporte de los acoplamientos a los cables de conexión, que podría ser dominante, se vuelve despreciable. En estas condiciones, la principal contribución de EMI se debe a la corriente de desplazamiento $i_{\mathrm{P}}$ que ingresa al paciente y el modelo de interferencia se reduce al esquema de la Fig.3.1. Este modelo simple, pero suficiente para analizar el problema, es el utilizado habitualmente en la literatura (THAKOR \& WEBSTER, 1980), (PALlas-ARENY, 1986).

El circuito equivalente de la Fig.3.1 desprecia la capacidad línea-común $C_{\text {SUP. }}$ Esto es razonable dado que los sistemas dos electrodos se utilizan, casi exclusivamente, en pequeños amplificadores portátiles alimentados a baterías.

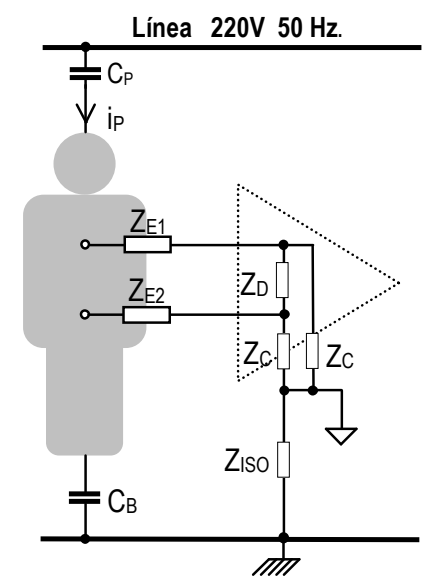

Figura 3.1. Modelo de Interferencia de la red en sistemas de dos electrodos.

Si se considera una señal biológica nula, la tensión de salida del amplificador se deberá exclusivamente a la interferencia de la red $(50 \mathrm{~Hz})$. Refiriendo esta tensión a la entrada del amplificador como $V_{\text {iD.EMI }}$ y expresándola a partir de las tensiones de modo común $V_{\mathrm{iC}} \mathrm{y}$ de modo diferencial $V_{\mathrm{iD}}$ presentes a la entrada del amplificador resulta ${ }^{1}$ :

$$
V_{\mathrm{iD.EMI}}=V_{\mathrm{iD}}+\frac{V_{\mathrm{iC}}}{\mathrm{CMRR}}
$$

\footnotetext{
${ }^{1}$ En esta expresión fue despreciado el efecto de la tensión de modo aislante $V_{\text {ISO }}$. Incluyendo este aporte, la ecuación (3.1) se transformaría en $V_{\mathrm{iD} . \mathrm{EMI}}=V_{\mathrm{iD}}+V_{\mathrm{iC}} / \mathrm{CMRR}+V_{\mathrm{ISO}} / \mathrm{IMRR}$. Actualmente, el esquema más usual consiste en preamplificar la señal utilizando un A.B. alimentado a baterías y atravesar la barrera de aislación en forma digital, resultando IMRR muy elevados que permiten despreciar los efectos de $V_{\text {ISO }}$ (WOOD, 1995)(MEETING VAN RIJN, 1991)
} 
La tensión de interferencia $V_{\text {iD.EMI }}$ depende fuertemente de la impedancia de entrada de modo común $Z_{\mathrm{C}}$. Este comportamiento ha sido analizado para valores moderados $\mathrm{y}$ altos de $Z_{\mathrm{C}}$ (ThaKor, 1980),(PALlas-Areny, 1986), (PALlAS-ARENY, 1988), mostrando que una alta $Z_{C}$ no es necesariamente la mejor elección.

Para observar cualitativamente la dependencia de $V_{\text {iD.EMI }}$ con $\mathrm{Z}_{\mathrm{C}}$ resulta suficiente el modelo simplificado ${ }^{2}$, válido para tensiones de modo común, que se presenta en la Fig. 3.2.

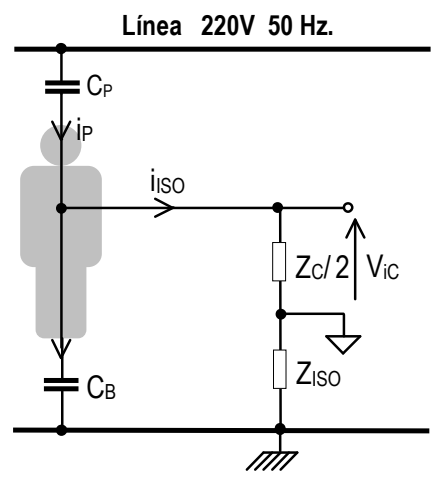

Figura 3.2. Circuito simplificado de modo común para analizar cualitativamente la dependencia de la tensión de interferencia con $Z_{c}$.

Se observa aquí que una fracción de la corriente $i_{\mathrm{P}}$ indicada como $i_{\mathrm{ISO}}$, circulará por $Z_{\mathrm{C}}$. La diferencia de potencial que esta corriente produce sobre $Z_{\mathrm{C}}$ define la tensión de modo común $V_{\text {ic }}$ presente en la entrada del A.B.

Si la impedancia de modo común $Z_{\mathrm{C}}$ es baja, la tensión $V_{\mathrm{iC}}$ será reducida, pero debido al efecto "divisor de potencial" (APÉNDICE 2), $V_{\text {iD.EMI }}$ será importante. Por otro lado, si $Z_{\mathrm{C}}$ es elevada, la tensión de modo común $V_{\text {iC }}$ puede ser muy alta (de hasta decenas de volts $^{3}$ ) y el CMRR finito del A.B. conduciría a niveles apreciables de interferencia a la salida del A.B. Como puede advertirse, estos dos efectos están contrapuestos. Es posible que se compensen conduciendo a una $V_{\text {iD.EMI }}$ insensible a $Z_{\mathrm{C}}$, que exista un valor óptimo de $Z_{\mathrm{C}}$ o que alguno de estos efectos sea dominante: el valor de $Z_{\mathrm{C}}$ más conveniente no es obvio y requiere un análisis detallado.

En la implementación de A.B. para sistemas de dos electrodos, "históricamente" se utilizó una $Z_{\mathrm{C}}$ tan elevada como fuera posible (THAKOR, 1980). Recientemente (DOBREV, 2002a), (DOBREV, 2002b) han propuesto un circuito que permite utilizar muy bajas $Z_{\mathrm{C}}$ (de solo algunos $\mathrm{k} \Omega$ ), manteniendo elevadas impedancias de modo diferencial $Z_{\mathrm{D}}$. Si bien estos autores no analizaron las características de rechazo a la tensión de red de esta estrategia, sí mostraron que la misma es posible y que ambas técnicas (altas y bajas $Z_{\mathrm{C}}$ ) son factibles. En el análisis que sigue se analizará cual es el valor de $Z_{\mathrm{C}}$ que conduce a mínima interferencia.

\footnotetext{
2 Para llegar a este modelo se consideraron impedancias de electrodo despreciables y una impedancia de entrada de modo diferencial $Z_{\mathrm{D}}$ infnita.

${ }^{3}$ Esto puede además comprometer el rango de entrada del A.B. Recordar que en mediciones con 3 electrodos $V_{\mathrm{CM}}$ difícilmente supera algunas centenas de $\mathrm{mV}$.
} 


\section{Modelo de Impedancias de Entrada.}

El modelo "clásico" para describir las impedancias de entrada de un amplificador (THAKOR, 1980); (PALLAS-ARENY, 1986); (PALLAS-ARENY 1988), que se muestra en la Fig.3.3.(a), no permite describir amplificadores con bajas $Z_{\mathrm{C}}$. Si $Z_{\mathrm{C}}$ tiende a cero, la entrada queda cortocircuitada y la señal no ingresaría al amplificador. Para extender el análisis a las estrategias que utilizan bajas $Z_{\mathrm{C}}$, es necesario reformular un nuevo modelo para las impedancias de entrada del amplificador.

En la Fig. 3.3.(b), se propone un modelo de acuerdo al circuito práctico propuesto por (DOBREV, 2002a). Este circuito equivalente permite modelar bajas $Z_{\mathrm{C}}$ manteniendo elevadas impedancias de entrada de modo diferencial $Z_{\mathrm{D}}$. Otra topología posible, que se propone en esta tesis, es la indicada en la Fig.3.3.(c). En el análisis que sigue, se considera esta última alternativa porque resulta más simple de implementar. De todos modos, ambas estrategias conducen a resultados similares (ver APÉNDICE 3) y a idénticas conclusiones.

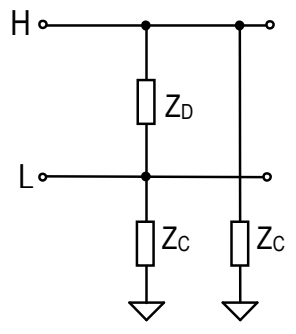

(a)

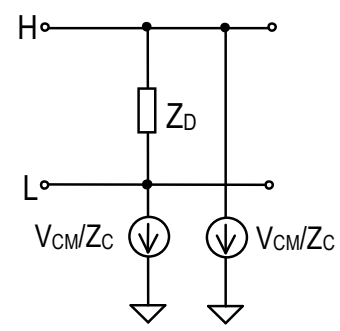

(b)

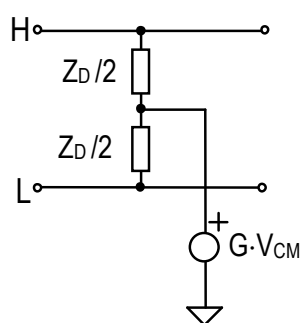

(c)

Figura 3.3. (a) Modelo "clásico" para las impedancias de entrada de un amplificador. (b) y (c) Dos modelos alternativos para describir estrategias que utilicen bajas impedancias de modo común.

La impedancia de entrada $Z_{\mathrm{C}}$ que el circuito de la Fig.3.3.(c), presenta para tensiones de modo común está dada por:

$$
Z_{\mathrm{C}}=\frac{Z_{\mathrm{D}} / 2}{1-G}
$$

Esta ecuación muestra que es posible controlar el valor de $Z_{\mathrm{C}}$ actuando sobre la ganancia de realimentación $G$. Así, tomando una ganancia unitaria, se obtiene una muy elevada $Z_{\mathrm{C}}$, mientras que utilizando una ganancia $G$ negativa y muy elevada, es posible conseguir muy bajas $Z_{\mathrm{C}}$. En ambos casos, la impedancia de entrada de modo diferencial es igual a $Z_{\mathrm{D}}$.

\section{Cálculo de la tensión de interferencia.}

Utilizando como modelo para la etapa de entrada el circuito equivalente propuesto en la Fig.3.3.(c), el modelo de interferencia de la Fig.3.1 puede llevarse al circuito de la Fig.3.4. En este esquema, la impedancia indicada como $Z_{\mathrm{B}}$ corresponde a la reactancia de la capacidad paciente-tierra $C_{\mathrm{B}}$ evaluada a la frecuencia de red $(50 \mathrm{~Hz})$. 


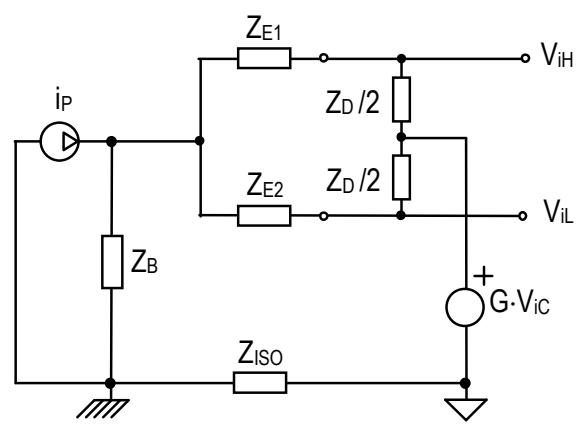

Figura 3.4. Circuito equivalente del modelo de interferencia de la Fig.3.1, asumiendo el modelo de impedancias de entrada de la Fig.3.3.c.

Resolviendo este circuito (APÉNDICE 3), resultan las siguientes expresiones para las tensiones de modo común $V_{\text {iC }}$ y modo diferencial $V_{\text {iD }}$ a la entrada del A.B.

$$
\begin{gathered}
V_{\mathrm{iC}}=i_{\mathrm{P}} \frac{Z_{\mathrm{B}} Z_{\mathrm{C}}}{2\left(Z_{\mathrm{B}}+Z_{\mathrm{ISO}}\right)+Z_{\mathrm{C}}} \\
V_{\mathrm{iD}}=i_{\mathrm{P}} \frac{Z_{\mathrm{B}}\left(Z_{\mathrm{E} 1}-Z_{\mathrm{E} 2}\right)}{2\left(Z_{\mathrm{B}}+Z_{\mathrm{ISO}}\right)+Z_{\mathrm{C}}}
\end{gathered}
$$

Estas expresiones son válidas para cualquier $Z_{\mathrm{C}}$ siempre que se cumpla que $\mathrm{Z}_{\mathrm{E} 1}, \mathrm{Z}_{\mathrm{E} 2}$ " $Z_{D}$. Esta condición debe ser satisfecha por cualquier sistema de medida de biopotenciales razonable, a fin de no atenuar la señal bioeléctrica.

Reemplazando (3.3) y (3.4) en (3.1) resulta:

$$
V_{\mathrm{iD} . \mathrm{EMI}}=i_{\mathrm{P}} \frac{Z_{\mathrm{B}}}{Z_{\mathrm{C}}+2\left(Z_{\mathrm{B}}+Z_{\mathrm{ISO}}\right)}\left(\Delta Z_{\mathrm{E}}+\frac{Z_{\mathrm{C}}}{\mathrm{CMRR}}\right)
$$

donde $\Delta Z_{\mathrm{E}}=Z_{\mathrm{E} 1}-Z_{\mathrm{E} 2}$ representa el desbalance absoluto entre las impedancias de electrodo.

Para disponer de un parámetro representativo de la condición de EMI y al mismo tiempo independiente de la corriente de desplazamiento $i_{\mathrm{P}}$, se define una Impedancia Efectiva de Acoplamiento $Z_{\mathrm{CE}}$ (PALLAS-ARENY, 1988), la cual permite rescribir (3.5) como

$$
V_{\mathrm{iD.EMI}}=i_{\mathrm{P}} Z_{\mathrm{CE}}
$$

Según se desprende de (3.6), para conseguir un buen rechazo a fuentes de EMI, es deseable un bajo valor de $Z_{\mathrm{CE}}$. Comparando (3.5) con (3.6) se obtiene, para el caso de medición con 2 electrodos, la siguiente expresión de $Z_{\mathrm{CE}}$ :

$$
Z_{C E}=\frac{Z_{B}}{Z_{C}+2\left(Z_{B}+Z_{I S O}\right)}\left(\Delta Z_{E}+\frac{Z_{C}}{\mathrm{CMRR}}\right)
$$




\subsection{Mínima Impedancia de Acoplamiento Efectiva}

Para reducir la tensión de interferencia $V_{\text {iD.EMI}}$, se requiere minimizar el módulo de $Z_{\mathrm{CE}}$. Considerando que $Z_{\mathrm{B}}$ y $Z_{\mathrm{ISO}}$ son ambas capacitivas, es decir $Z_{\mathrm{B}}=-\mathrm{j} X_{\mathrm{B}}$ y $Z_{\mathrm{ISO}}=-\mathrm{j} X_{\mathrm{ISO}}$, la ecuación (3.7) puede escribirse como:

$$
Z_{\mathrm{CE}}=\frac{-\mathrm{j} X_{\mathrm{B}}}{Z_{\mathrm{C}}-\mathrm{j} 2\left(X_{\mathrm{B}}+X_{\mathrm{ISO}}\right)}\left(\Delta Z_{\mathrm{E}}+\frac{Z_{\mathrm{C}}}{\mathrm{CMRR}}\right)
$$

El peor caso (el mayor módulo de $\mathrm{Z}_{\mathrm{CE}}$ ), se produce cuando los sumandos en el segundo factor están en fase. En estas condiciones (3.8) se transforma en:

$$
Z_{\mathrm{CE}}=\frac{-\mathrm{j} X_{\mathrm{B}}}{Z_{\mathrm{C}}-\mathrm{j} 2\left(X_{\mathrm{B}}+X_{\mathrm{ISO}}\right)}\left(\left|\Delta Z_{\mathrm{E}}\right|+\frac{\left|Z_{\mathrm{C}}\right|}{|\mathrm{CMRR}|}\right)
$$

Para reducir esta impedancia se requiere $X_{\mathrm{B}} \rightarrow 0 ; \Delta Z_{\mathrm{E}} \rightarrow 0 ; X_{\mathrm{ISO}} \rightarrow \infty$ y CMRR $\rightarrow \infty$, pero el valor de $Z_{\mathrm{C}}$ para conseguir la mínima $Z_{\mathrm{CE}}$ no es obvio a partir de (3.9). Para reducir el primer factor se requiere un alto valor de $Z_{C}$ de tipo capacitivo, mientras que el segundo factor decae para bajos valores de $Z_{\mathrm{C}}$.

La impedancia efectiva de acoplamiento $Z_{\mathrm{CE}}$ es una función de la variable compleja $Z_{\mathrm{C}}$. El valor de $Z_{\mathrm{C}}$ que produce el mínimo módulo de $Z_{\mathrm{CE}}$ puede determinarse aplicando el principio de mínimo módulo (KRANTZ, 1999). Este principio establece lo siguiente:

"Dada una función $f$, analítica en un domino cerrado $U$, si $f$ no se anula en ningún punto dentro de este dominio, entonces el mínimo de $|f|$ se produce sobre el contorno de $U$."

De este modo, el problema de determinar el mínimo de $\left|Z_{\mathrm{CE}}\right|$ sobre una región puede reducirse a encontrarlo sobre una curva cerrada.

Con el fin de encontrar el valor de $Z_{\mathrm{C}}$ que conduce a la mínima $Z_{\mathrm{CE}}$, se adoptó una región cerrada $U$ que cubre todos los valores de $Z_{\mathrm{C}}$ con parte real positiva y con parte imaginaria negativa (capacitiva) (ver Fig.3.5). Es simple verificar que $Z_{\mathrm{CE}}\left(Z_{\mathrm{C}}\right)$ es analítica dentro de esta región: su polo en $Z_{\mathrm{C}}=+2 \mathrm{j}\left(X_{\mathrm{B}}+X_{\mathrm{ISO}}\right)$ queda fuera de este dominio y el origen, donde $\left|Z_{\mathrm{C}}\right|$ no es analítica, es evitado rodeándolo con un arco de radio tendiendo a cero. Es entonces posible aplicar del principio antes mencionado.

Los valores de $Z_{\mathrm{C}}$ con parte real negativa se ignoraron pues no tienen sentido físico. Tampoco se consideraron valores con parte imaginaria positiva (inductiva), dado que de acuerdo con (3.9) conducen ${ }^{4}$ a altos valores de $Z_{\mathrm{CE}}$, constituyendo una región de operación a priori no recomendable.

\footnotetext{
${ }^{4}$ Una $Z_{\mathrm{C}}$ inductiva reduce el denominador del primer factor de (3.9) incrementando $\left|Z_{\mathrm{CE}}\right|$.
} 


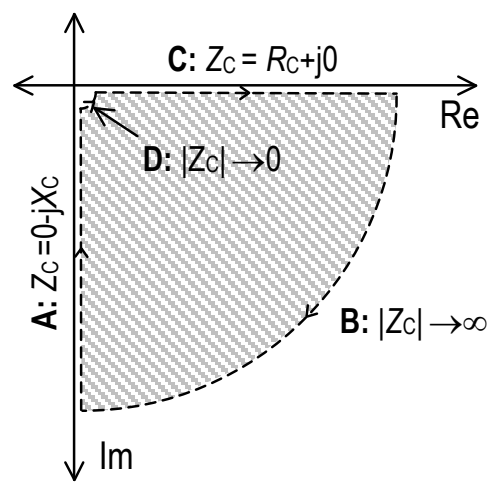

Figura 3.5. Región utilizada para determinar el valor de $Z_{c}$ que conduce a la mínima tensión de interferencia. El mínimo se encuentra sobre el contorno de esta región.

El contorno de la región $U$ adoptada está compuesto por cuatro curvas simples que serán analizadas por separado: $\mathrm{A}: Z_{\mathrm{C}}=0-\mathrm{j} X_{\mathrm{C}} ; \mathrm{B}:\left|Z_{\mathrm{C}}\right| \rightarrow \infty ; \mathrm{C}: Z_{\mathrm{C}}=R_{\mathrm{C}}+\mathrm{j} 0 ; \mathrm{y} \mathrm{D}:\left|Z_{\mathrm{C}}\right| \rightarrow 0$.

\subsubsection{Curva $A: Z_{C}=0-j X_{C}$ (capacitiva).}

Imponiendo la condición $Z_{\mathrm{C}}=0-\mathrm{j} X_{\mathrm{C}}$ en (3.9), la impedancia de acoplamiento efectiva resulta:

$$
\mid Z_{\mathrm{CE}} \|_{Z_{\mathrm{C}=-\mathrm{j} X_{\mathrm{C}}}}=\frac{X_{\mathrm{B}}}{X_{\mathrm{C}}+2\left(X_{\mathrm{B}}+X_{\mathrm{ISO}}\right)}\left(\left|\Delta Z_{\mathrm{E}}\right|+\frac{X_{\mathrm{C}}}{|\mathrm{CMRR}|}\right),
$$

cuya derivada está dada por:

$$
\frac{\partial \mid Z_{\mathrm{CE}} \|_{Z_{\mathrm{C}}=j X_{\mathrm{C}}}}{\partial X_{\mathrm{C}}}=\frac{X_{\mathrm{B}}}{\left[X_{\mathrm{C}}+2\left(X_{\mathrm{B}}+X_{\mathrm{ISO}}\right)\right]^{2}}\left(-\left|\Delta Z_{\mathrm{E}}\right|+\frac{2\left(X_{\mathrm{B}}+X_{\mathrm{ISO}}\right)}{|\mathrm{CMRR}|}\right) .
$$

Esta derivada no se anula para ningún valor finito de $X_{\mathrm{C}}$, es decir que sobre $Z_{\mathrm{C}}=0-\mathrm{j} X_{\mathrm{C}}$ la impedancia de acoplamiento efectiva no presenta ningún mínimo local y el mínimo módulo de $Z_{\mathrm{CE}}$ se producirá para valores extremos de $X_{\mathrm{C}}$. El signo de la derivada (3.11), está dado por:

$$
\operatorname{Sign}\left(\frac{\partial \mid Z_{\mathrm{CE}} \|_{Z_{\mathrm{C}}=\mathrm{j} X_{\mathrm{C}}}}{\partial X_{\mathrm{C}}}\right)=\operatorname{Sign}\left(-\left|\Delta Z_{\mathrm{E}}\right|+\frac{2\left(X_{\mathrm{B}}+X_{\mathrm{ISO}}\right)}{|\mathrm{CMRR}|}\right)
$$

y es independiente de $X_{\mathrm{C}}$. Si se verifica la condición:

$$
\left|\Delta Z_{\mathrm{E}}\right|<\frac{2\left(X_{\mathrm{B}}+X_{\mathrm{ISO}}\right)}{|\mathrm{CMRR}|} \Rightarrow \frac{\partial Z_{\mathrm{CE}}}{\partial X_{\mathrm{C}}}>0 \text { para todo } X_{\mathrm{C}}
$$


la función (3.10) es monótonamente creciente y el mínimo valor de $\left|Z_{\mathrm{CE}}\right|$ se produce para $X_{\mathrm{C}}=0$. Por el contrario, si se cumple que:

$$
\left|\Delta Z_{\mathrm{E}}\right|>\frac{2\left(X_{\mathrm{B}}+X_{\mathrm{ISO}}\right)}{|\mathrm{CMRR}|} \Rightarrow \frac{\partial Z_{\mathrm{CE}}}{\partial X_{\mathrm{C}}}<0 \text { para todo } X_{\mathrm{C}},
$$

la impedancia $Z_{\mathrm{CE}}$ resulta una función monótonamente decreciente de $X_{\mathrm{C}}$ y la mejor opción para conseguir la mínima $\left|Z_{\mathrm{CE}}\right|$ es $X_{\mathrm{C}} \rightarrow \infty$. Para distinguir entre estas condiciones, es posible definir un desbalance crítico $\Delta Z_{\mathrm{E} \text {.Crítico }}$ dado por:

$$
\Delta Z_{\mathrm{E} . \text { Critico }}=\frac{2\left(X_{\mathrm{B}}+X_{\mathrm{ISO}}\right)}{|\mathrm{CMRR}|}
$$

Entonces,

- Si $\Delta Z_{\mathrm{E}}>\Delta Z_{\mathrm{E} \text {.Crítico }}$, el mínimo módulo de $Z_{\mathrm{CE}}$ se alcanza para $X_{\mathrm{C}} \rightarrow \infty$.

- Si $\Delta Z_{\mathrm{E}}<\Delta Z_{\mathrm{E} \text {.Crítico }}$, el mínimo módulo de $Z_{\mathrm{CE}}$ se produce para $X_{\mathrm{C}}=0$.

Por ejemplo, considerando un sistema con los siguientes parámetros: $Z_{\mathrm{ISO}}=10 \mathrm{pF}$, CMRR@50Hz=90dB y $C_{\mathrm{B}}=150 \mathrm{pF}$, resulta un $\Delta Z_{\mathrm{E} \text {.Crítico }}$ de $21.5 \mathrm{k} \Omega$. En la Fig.3.6 se muestra, para este caso, $\left|Z_{\mathrm{CE}}\right|$ como función de $X_{\mathrm{C}}$ considerando $\Delta Z_{\mathrm{E}}=5 \mathrm{k} \Omega$ y $\Delta Z_{\mathrm{E}}=50 \mathrm{k} \Omega$. Como puede observarse, las condiciones de medida óptimas están dadas para valores extremos de $X_{\mathrm{C}}$. Si $\Delta Z_{\mathrm{E}}>\Delta Z_{\mathrm{E} \text {.Crítico }}$, la mínima $Z_{\mathrm{CE}}$ se alcanza para $X_{\mathrm{C}}=\infty$ y por el contrario, si $\Delta Z_{\mathrm{E}}<\Delta Z_{\mathrm{E} \text {. Critico }}$, el menor nivel de interferencia se alcanza con $X_{\mathrm{C}}=0$.

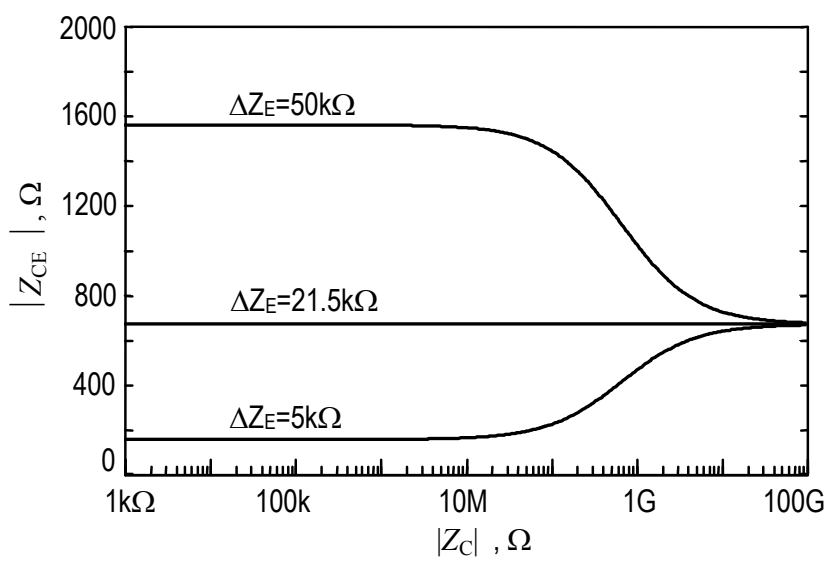

Figura 3.6. Impedancia de acoplamiento efectiva $\left(Z_{C E}\right)$ como función de $Z_{c}$ para $Z_{C}=0+j X_{c}$ y diferentes desbalances $\left(\Delta Z_{E}\right)$ en las impedancias de electrodo. Para bajos $\Delta Z_{E}$, el mínimo $Z_{C E}$ se produce para $X_{C}=0$, mientras que para elevados $\Delta Z_{E}$ la mejor condición es $X_{C} \rightarrow \infty$.

\subsubsection{Curva C: $Z_{\mathrm{C}}=R_{\mathrm{C}}+\mathrm{j} 0$.}

Imponiendo la condición $Z_{\mathrm{C}}=R_{\mathrm{C}}$ en (3.9), la expresión de $\left|Z_{\mathrm{CE}}\right|$ se transforma en: 


$$
\left|Z_{\mathrm{CE}}\right|_{Z_{\mathrm{C}}=R_{\mathrm{C}}}=\frac{X_{\mathrm{B}}}{\left(R_{\mathrm{C}}^{2}+4\left(X_{\mathrm{B}}+X_{\mathrm{ISO}}\right)^{2}\right)^{\frac{1}{2}}}\left(\left|\Delta Z_{\mathrm{E}}\right|+\frac{R_{\mathrm{C}}}{|\mathrm{CMRR}|}\right),
$$

y su derivada resulta:

$$
\frac{\partial \mid Z_{\mathrm{CE}} \|_{Z_{\mathrm{C}}=R_{\mathrm{C}}}}{\partial R_{\mathrm{C}}}=\frac{X_{\mathrm{B}}}{\left(R_{\mathrm{C}}^{2}+4\left(X_{\mathrm{B}}+X_{\mathrm{ISO}}\right)^{2}\right)^{\frac{3}{2}}}\left(\frac{4\left(X_{\mathrm{B}}+X_{\mathrm{ISO}}\right)^{2}}{|\mathrm{CMRR}|}-R_{\mathrm{C}}\left|\Delta Z_{\mathrm{E}}\right|\right) .
$$

Esta derivada se anula para $R_{\mathrm{C}}=R_{\mathrm{C} 0}$, dado por:

$$
R_{\mathrm{C} 0}=\frac{4\left(X_{\mathrm{B}}+X_{\mathrm{ISO}}\right)^{2}}{|\operatorname{CMRR}|\left|\Delta Z_{\mathrm{E}}\right|}
$$

Para determinar si $R_{\mathrm{C}}=R_{\mathrm{C} 0}$ corresponde a un mínimo o a un máximo, es necesario inspeccionar el signo de la derivada segunda en este punto. Derivando (3.17) y evaluando su signo en $R_{\mathrm{C}}=R_{\mathrm{C} 0}$ resulta:

$$
\left.\operatorname{Sign}\left(\frac{\partial^{2}\left|Z_{\mathrm{CE}}\right|_{Z_{\mathrm{C}}=R_{\mathrm{C}}}}{\partial^{2} R_{\mathrm{C}}}\right)\right|_{R_{\mathrm{C}}=\mathrm{R}_{\mathrm{C} 0}}=\operatorname{Sign}\left(-\left|\Delta Z_{\mathrm{E}}\right|\right)<0
$$

Es decir que la derivada segunda en $R_{\mathrm{C} 0}$ es incondicionalmente negativa. Esto significa que la expresión (3.16) presenta un máximo en $R_{\mathrm{C}}=R_{\mathrm{C} 0}$ y no posee ningún mínimo local. El mínimo valor de $\left|Z_{\mathrm{CE}}\right|$ se producirá para valores extremos de $R_{\mathrm{C}}$.

En la Fig.3.7 se muestra el módulo de $Z_{\mathrm{CE}}$ para $Z_{\mathrm{C}}=R_{\mathrm{C}}+\mathrm{j} 0$ utilizando el mismo juego de parámetros que en la sección previa. En esta figura se aprecia el máximo local y se observa también que las condiciones de medida optimas se producen para $R_{\mathrm{C}}=0 \mathrm{o}$ $R_{\mathrm{C}}=\infty$, adoptando los mismos valores mínimo y máximo que en la Fig.3.6.

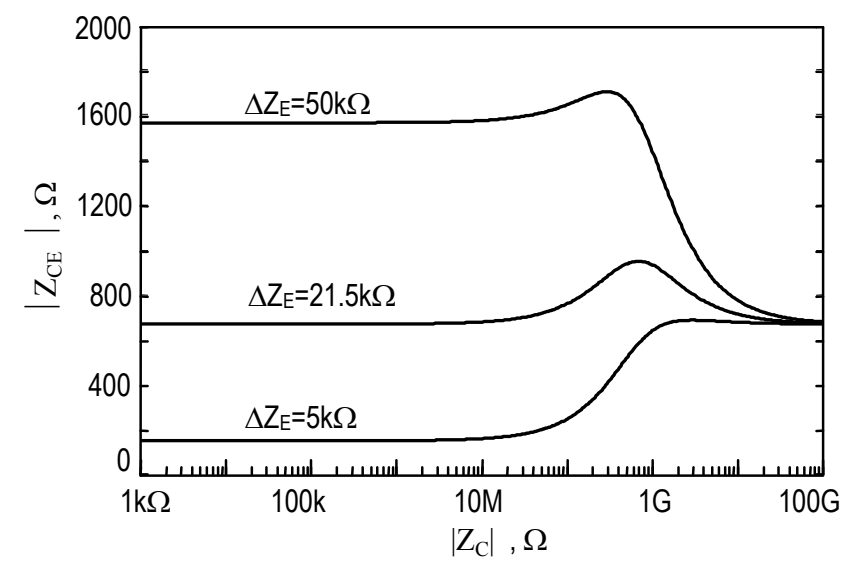

Figura 3.7. Impedancia de Acoplamiento Efectiva $Z_{C E}$ como función de $Z_{C}$ para $\mathrm{Z}_{\mathrm{C}}=\mathrm{R}_{\mathrm{C}}+\mathrm{j0}$ y distintos desbalances $\Delta \mathrm{Z}_{\mathrm{E}}$. 


\subsubsection{Curvas $D:\left|Z_{c}\right| \rightarrow 0$ y $B:\left|Z_{c}\right| \rightarrow \infty$.}

Sobre estas curvas, el modulo de $Z_{\mathrm{CE}}$ se vuelve constante. Si el módulo de $Z_{\mathrm{C}}$ es suficientemente grande, la ecuación (3.9) conduce a :

$$
\mid Z_{\mathrm{CE}} \|_{\left|Z_{\mathrm{C}}\right| \rightarrow \infty}=\frac{X_{\mathrm{B}}}{|\mathrm{CMRR}|}
$$

Por otro lado, si modulo el de $Z_{\mathrm{C}}$ tiende a cero, resulta:

$$
\mid Z_{\mathrm{CE}} \|_{\left|Z_{\mathrm{C}}\right| \rightarrow 0}=\frac{X_{\mathrm{B}}\left|\Delta Z_{\mathrm{E}}\right|}{2\left(X_{\mathrm{B}}+X_{\mathrm{ISO}}\right)}
$$

No existen mínimos ni máximos sobre estas curvas.

\subsection{Resultados del Análisis.}

A partir del análisis previo es posible concluir:

- La impedancia de acoplamiento efectiva $Z_{\mathrm{CE}}$ como función de $Z_{\mathrm{C}}$ no presenta ningún mínimo local.

- Si el desbalance $\Delta Z_{\mathrm{E}}$ entre las impedancias electródicas es reducido, la mejor opción es $Z_{\mathrm{C}}=0$, mientras que en presencia de elevadas $\Delta Z_{\mathrm{E}}$ es recomendable utilizar impedancias $Z_{\mathrm{C}}$ capacitivas y tan elevadas como sea posible $\left(X_{\mathrm{C}}=\infty\right)$.

- Una $Z_{\mathrm{C}}$ inductiva no es recomendable pues conduce a elevadas $Z_{\mathrm{CE}}$.

- Considerando una $Z_{\mathrm{C}}$ resistiva $\left(Z_{\mathrm{C}}=R_{\mathrm{C}}\right)$, el mínimo de $Z_{\mathrm{CE}}$ se produce para valores extremos de $R_{\mathrm{C}}$ y presenta un máximo local.

\section{Diseño para Mínima Interferencia de $50 \mathrm{~Hz}$.}

Como puede verse a partir de (3.9), para reducir la impedancia de acoplamiento efectiva $Z_{\mathrm{CE}}$ (y consecuentemente la interferencia de la red), el esfuerzo de diseño del amplificador debe focalizarse en conseguir altos CMRR e impedancias de aislamiento $Z_{\text {ISo }}$ tan altas como sea posible. Sería también deseable una alta capacidad pacientetierra $C_{\mathrm{B}}$, pero esto escapa del dominio del diseño.

La conveniencia de una muy alta o una muy baja $Z_{\mathrm{C}}$ depende de los máximos valores de CMRR y $Z_{\text {ISo }}$ alcanzables con los dispositivos disponibles. Dados estos valores, considerando un valor típico de $C_{\mathrm{B}}$ (digamos unos $200 \mathrm{pF}$ ), el valor de $\Delta Z_{\mathrm{E} \text {.Crítico }}$ puede ser calculado mediante (3.15). Luego, si el desbalance de impedancias de electrodo $\Delta Z_{\mathrm{E}}$ esperado es menor que el crítico (esto es $\Delta Z_{\mathrm{E}}<\Delta Z_{\mathrm{E} \text {.Crítico), una estrategia de baja }}$ $Z_{\mathrm{C}}$ conducirá a la mínima interferencia posible para las condiciones dadas. Por otro lado, si $\Delta Z_{\mathrm{E}}>\Delta Z_{\mathrm{E} \text {.Crítico }}$, el nivel de interferencia será mínimo utilizando una $Z_{\mathrm{C}}$ tan alta como sea posible.

Si se adopta una estrategia de baja $Z_{\mathrm{C}}$, el CMRR del A.B. no tiene importancia. Como se observa en (3.21), en este caso, la impedancia $Z_{\mathrm{CE}}$ se vuelve independiente del CMRR. 


\subsection{Consideraciones Prácticas.}

Actualmente, valores de CMRR de $100 \mathrm{~dB}$ o mayores no son difíciles de conseguir. Por otro lado, la técnica de "dos-electrodos" usualmente se utiliza en pequeños amplificadores portátiles alimentados a batería. Estos amplificadores generalmente presentan un alto nivel de aislamiento $\left(C_{\mathrm{ISO}}\right.$ menores de $\left.10 \mathrm{pF}\right)$, resultando valores de $\Delta Z_{\text {E.Crítico }}$ de unos $10 \mathrm{k} \Omega$.

De acuerdo a (RosSEL, 1988), las impedancias electrodo-piel, a la frecuencia de red, adoptan valores entre $10 \mathrm{k} \Omega$ y $1 \mathrm{M} \Omega$, siendo habitual encontrar desbalances $\Delta Z_{\mathrm{E}}$ mayores de $10 \mathrm{k} \Omega$. En estas condiciones, una alta $Z_{C}$ es la mejor alternativa para conseguir el menor nivel de interferencia.

En la práctica, esta mejora no es muy significativa. Como puede observarse en las Fig.3.6 y Fig. 3.7, $Z_{\mathrm{CE}}$ permanece aproximadamente constante para un amplio rango de valores de $Z_{C}$. Si el desbalance $\Delta Z_{E}$ es elevado, $Z_{C E}$ decrecerá con $Z_{C}$, pero este efecto solo es notable para valores muy altos de $Z_{\mathrm{C}}$; del orden de $Z_{\mathrm{C}} \approx-\mathrm{j} \Delta Z_{\mathrm{E}} \cdot \mathrm{CMRR}$.

Considerando un CMRR de $100 \mathrm{~dB}$, los valores de $Z_{\mathrm{C}}$ para los cuales $Z_{\mathrm{CE}}$ se torna significativamente dependiente de $Z_{\mathrm{C}}$ resultan de varias centenas de $\mathrm{M} \Omega$. Estos valores de $Z_{\mathrm{C}}$ no son simples de alcanzar en un sistema de adquisición de biopotenciales estándar, teniendo en cuenta que las protecciones de sus entradas, así también como los cables de conexión blindados, degradan la impedancia de entrada para señales de modo común. En general, las condiciones de operación corresponden a una región donde $Z_{\mathrm{CE}}$ es poco sensible a $Z_{\mathrm{C}}$ y tanto una alta como una baja $Z_{\mathrm{C}}$ conducen a similares rechazos de la interferencia de la red.

Ahora, si bien una alta $Z_{\mathrm{C}}$ usualmente conduce al menor nivel de interferencia, también produce elevadas tensiones de modo común $V_{\mathrm{CM}}$, lo cual puede comprometer el rango de entrada del A.B., degradando así las prestaciones del A.B.

\section{Verificación Experimental.}

El análisis efectuado a partir del modelo de la Fig.3.1 sugiere que las condiciones de medida óptimas, en cuanto a la impedancia de modo común $Z_{\mathrm{C}}$, se producen cuando la misma adopta valores extremos.

Con el fin de validar este resultado teórico, se diseñó una experiencia para obtener medidas experimentales de niveles de interferencia con diversos valores de $Z_{\mathrm{C}} \mathrm{y}$ distintos desbalances $\Delta Z_{\mathrm{E}}$. Un amplificador de biopotenciales, el cual se muestra en la Fig.3.8, fue especialmente diseñado para este ensayo. El circuito, utilizando una realimentación de modo común que se analiza en detalle en el CAPÍTULO 7, permite seleccionar entre una muy baja $Z_{\mathrm{C}}(\approx 100 \Omega)$ o una muy alta $Z_{\mathrm{C}}(700 \mathrm{M} \Omega)$. En ambos casos, la impedancia de entrada para señales de modo diferencial $Z_{\mathrm{D}}$ es de $20 \mathrm{M} \Omega$.

El A.B. se implementó utilizando un amplificador de instrumentación integrado INA111 y una etapa adicional con un circuito "sumador" que permite adicionar, en forma controlada, tensión de modo común. Esto permite seleccionar entre un CMRR de 90 o de $105 \mathrm{~dB}$ para obtener, según (3.15), distintos desbalances críticos $\Delta Z_{\mathrm{E}}$. 
El A.B. se alimentó con dos baterías de $9 \mathrm{~V}$ y se mantuvo aislado de tierra mediante un amplificador de aislación integrado ISO122. El circuito fue montado en un gabinete metálico simulando sus condiciones dentro de un Electrocardiógrafo.

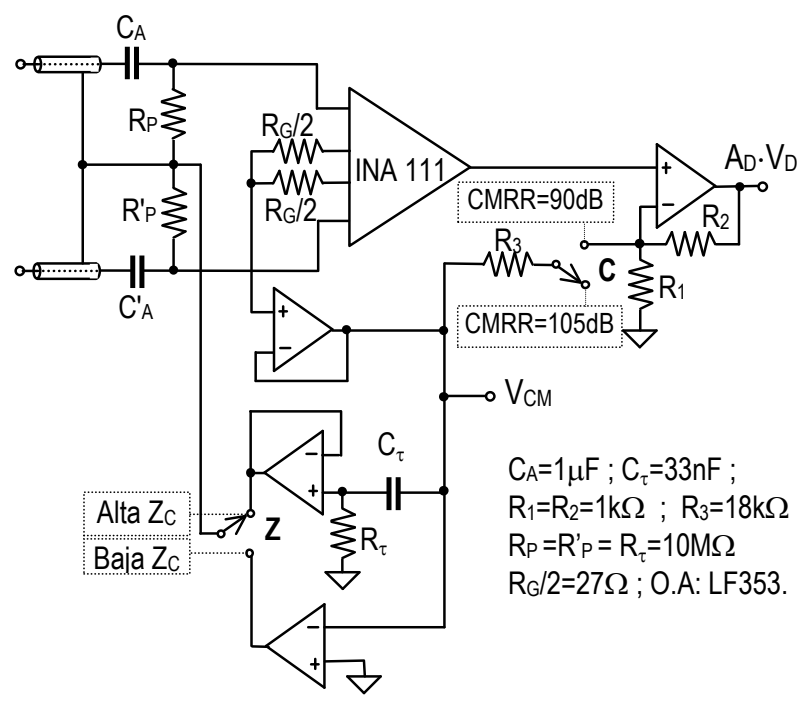

Figura 3.8. Amplificador de dos electrodos utilizado para validar las expresiones obtenidas. Mediante la llave "Z" es posible seleccionar una muy alta 0 una muy baja $\mathrm{Zc}$. El circuito también permite, mediante la llave "C", seleccionar entre dos valores de CMRR que determinan, según (3.15) distintos desbalances críticos $\Delta \mathrm{Z}_{\mathrm{E} \text {.Critico. }}$

\subsection{Estimación de la impedancia de acoplamiento efectiva $Z_{C E}$.}

En primer lugar, para verificar los resultados analíticos con desbalances $\Delta Z_{\mathrm{E}}$ bien conocidos, se utilizó el esquema que se muestra en la Fig.3.9. Se colocó un único electrodo sobre el paciente y se insertó un resistor variable en serie con una de las entradas del A.B. De este modo, $\Delta Z_{\mathrm{E}}$ esta dado precisamente por el valor del resistor utilizado.

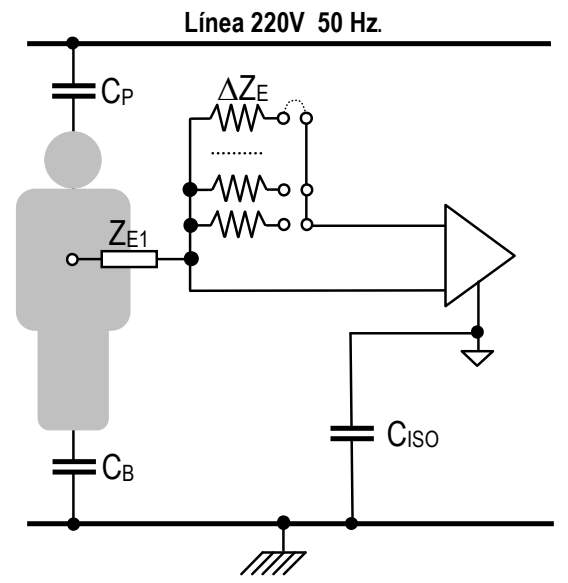

Figura 3.9. Esquema utilizado para medir con desbalances $\Delta Z_{E}$ conocidos. 
Se realizó un primer ensayo fue realizado utilizando un $C M R R=90 \mathrm{~dB}$ y diferentes desbalances en la impedancia electrodo-piel simulados mediante resistores $\Delta Z_{\mathrm{E}}=5 \mathrm{k} \Omega$, $10 \mathrm{k} \Omega, 20 \mathrm{k} \Omega, 40 \mathrm{k} \Omega$ y $60 \mathrm{k} \Omega$. La corriente $i_{\mathrm{P}}$ fue estimada de acuerdo a (HUHTA \& WEBSTER, 1973) resultando de $105 \mathrm{nA}$.

Para cada uno de los $\Delta \mathrm{Z}_{\mathrm{E}}$, se determinó la tensión diferencial de salida $V_{\text {iD.EMI }}$ para la más alta $Z_{C}$ alcanzada $(700 \mathrm{M} \Omega)$ y para una baja $Z_{C}(100 \Omega)$. En cada medida la impedancia de acoplamiento efectiva $Z_{\mathrm{CE}}$ se calculó como $Z_{\mathrm{CE}}=V_{\mathrm{iD.EMI}} / i_{\mathrm{P}}$, resultando las medidas que se presentan en la Fig.3.10. Se observa que para $\Delta Z_{\mathrm{E}}$ por debajo de $20 \mathrm{k} \Omega$, la menor interferencia se produce para una baja $Z_{C}$ mientras que esta situación se invierte para $\Delta Z_{\mathrm{E}}$ mayores de $40 \mathrm{k} \Omega$. Esto indica que el $\Delta \mathrm{Z}_{\mathrm{E} \text {.Crítico }}$ se encuentra entre estos valores, es decir que para este caso es de unos $30 \mathrm{k} \Omega$.

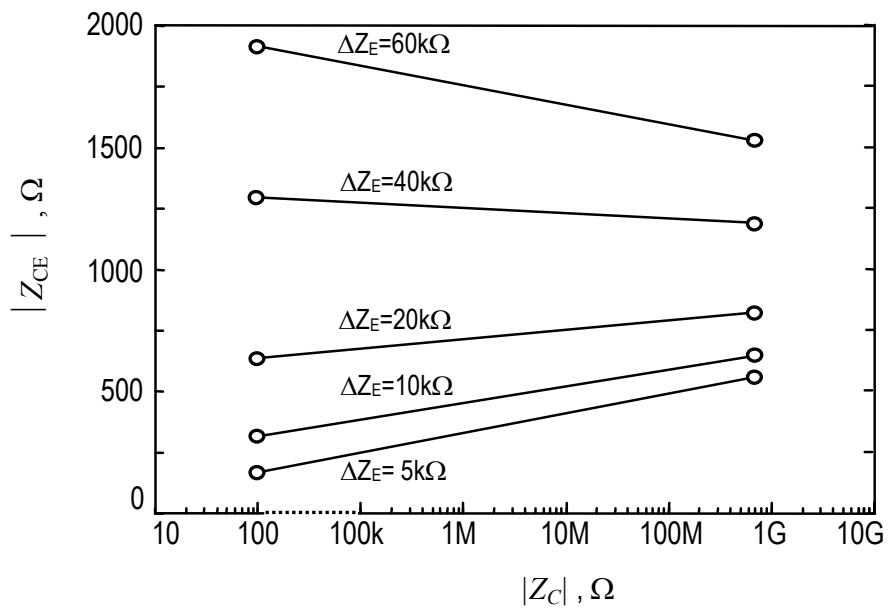

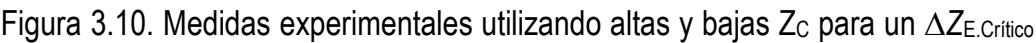
de $30 \mathrm{k} \Omega$.

\subsection{Registro de ECG.}

Un segundo juego de experiencias se realizó adquiriendo señales de ECG reales. Dos electrodos tipo "placa" de $12 \mathrm{Cm}^{2}$ se colocaron en los brazos derechos e izquierdo del paciente, es decir conformando la derivación I de ECG clínico. Previamente, la piel fue preparada mediante abrasión con una lija suave, consiguiendo valores de $\Delta Z_{\mathrm{E}}$ menores de $200 \Omega$. Esto fue verificado utilizando un método de medida de $\Delta Z_{\mathrm{E}}$ que se propone y detalla en el CAPítUlo 4. Los distintos valores de $\Delta Z_{\mathrm{E}}$ se produjeron insertando resistores en serie con uno de los electrodos.

Se realizó un primer ensayo utilizando un CMRR de $90 \mathrm{~dB}$ y manteniendo las mismas condiciones que en el punto anterior (un $\Delta Z_{\mathrm{E} \text {.Crítico }}$ de aproximadamente $30 \mathrm{k} \Omega$.). Los registros obtenidos para $\Delta Z_{\mathrm{E}}=5 \mathrm{k} \Omega$ y $\Delta Z_{\mathrm{E}}=60 \mathrm{k} \Omega$ utilizando una alta $(700 \mathrm{M} \Omega)$ y una baja $Z_{\mathrm{C}}(100 \Omega)$ se muestran en la Fig.3.11. Se observa aquí claramente que, para $\Delta Z_{\mathrm{E}}$ $<\Delta Z_{\text {E.Critico }}$, el menor nivel de interferencia se consigue con una baja $Z_{\mathrm{C}}$, mientras que si $\Delta Z_{\mathrm{E}}>\Delta Z_{\mathrm{E} \text {.Crítico }}$ la interferencia es menor utilizando una estrategia de alta $Z_{\mathrm{C}}$. 
Un segundo ensayo, con señales de ECG reales se realizó utilizando esta vez un CMRR@50Hz de 105dB. Al aumentar el CMRR, de acuerdo a (3.15) el desbalance crítico decrece en igual proporción. En este caso, el nuevo $\Delta Z_{\mathrm{E} \text {.Crítico }}$ resulta de alrededor de $5 \mathrm{k} \Omega$. Los registros obtenidos con altas y bajas $Z_{\mathrm{C}}$ para $\Delta Z_{\mathrm{E}} \approx 200 \Omega$ y $\Delta Z_{\mathrm{E}}=5 \mathrm{k} \Omega$ se muestran en la Fig.3.12. Como puede observarse, para $\Delta Z_{\mathrm{E}} \approx 200 \Omega$ (por debajo de $\Delta Z_{\mathrm{E} . \text { Crítico }}$ ), una baja $Z_{\mathrm{C}}$ conduce a un mejor rechazo de la interferencia de la tensión de línea, mientras que con un desbalance $\Delta Z_{\mathrm{E}}$ de $5 \mathrm{k} \Omega$ (próximo al valor crítico $\Delta Z_{\mathrm{E} \text {.Critico }}$ ), ambas estrategias muestran niveles de interferencia similares.

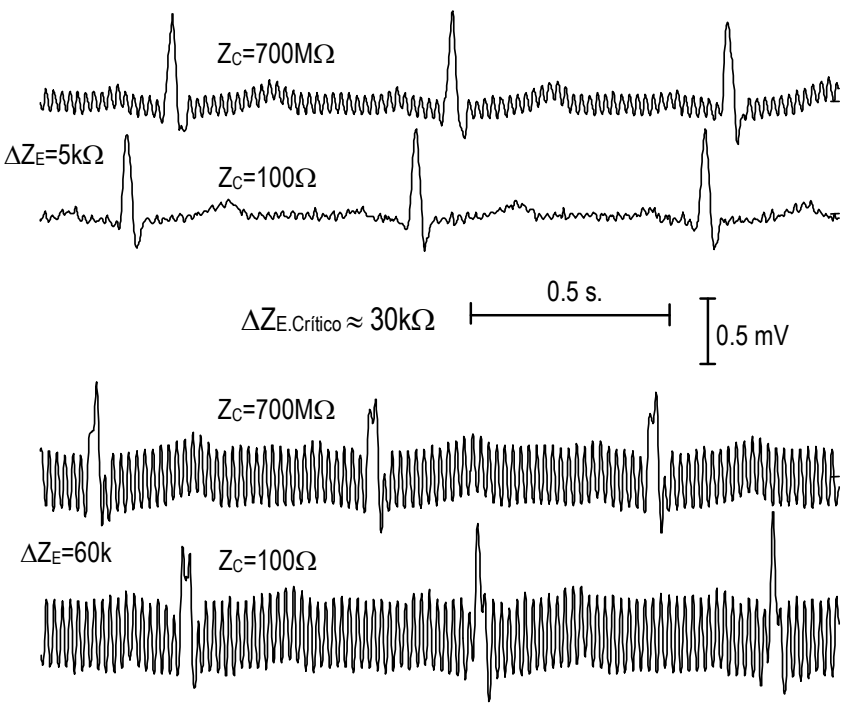

Figura 3.11. Registros de ECG obtenidos bajo un $\Delta$ zE.Critico $\approx 30 \mathrm{k} \Omega$ utilizando altas y bajas $Z_{C}$. Los trazos superiores corresponden a $\Delta Z_{E}=5$ $\mathrm{k} \Omega<\Delta \mathrm{Z}_{\mathrm{E} . \text {.rritico, }} \mathrm{y}$ los inferiores a $\Delta \mathrm{Z}_{\mathrm{E}}=60 \mathrm{k} \Omega>\Delta \mathrm{Z}_{\mathrm{E} \text {.Critico. }}$

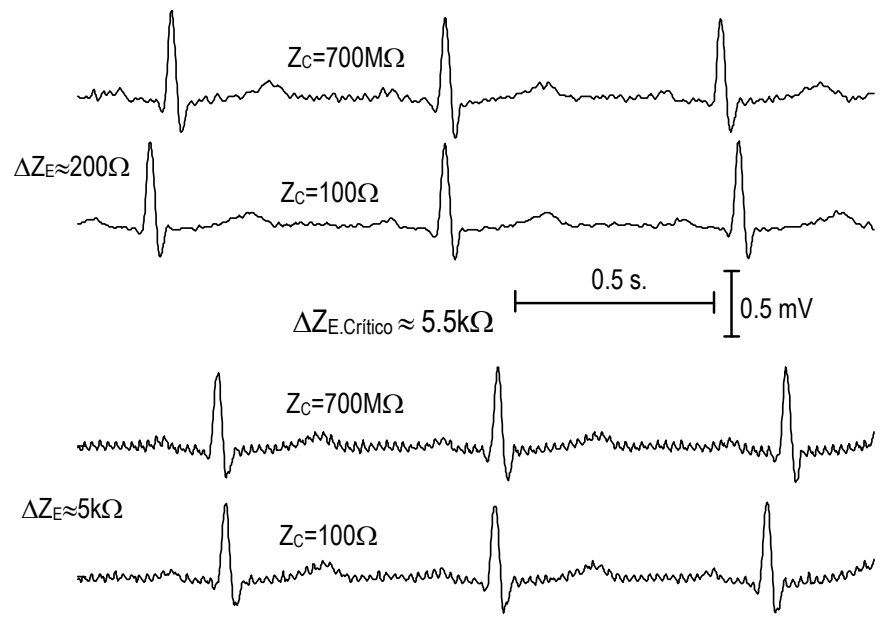

Figura 3.12. Registros de $E C G$ obtenidos bajo un $\Delta z$ z.Critico $\approx 5 \mathrm{k} \Omega$ utilizando altas y bajas $Z_{C}$. Los trazos superiores corresponden a $\Delta Z_{E}=200 \Omega<$ $\Delta \mathrm{Z}_{\mathrm{E} \text {.Critico }} \mathrm{y}$ los inferiores a $\Delta \mathrm{Z}_{\mathrm{E}}=5 \mathrm{k} \Omega \approx \Delta \mathrm{Z}_{\mathrm{E} \text {.Critico. }}$. 


\section{Conclusiones}

El nivel de interferencia introducido por la red de distribución $(50 \mathrm{~Hz})$ en sistemas de registro de biopotenciales que utilizan solo dos electrodos, es función de la impedancia de entrada $Z_{\mathrm{C}}$ que el A.B. presenta para tensiones de modo común. Esto puede expresarse en función de una impedancia de acoplamiento efectiva $Z_{\mathrm{CE}}$, la cual deber ser baja para reducir los efectos de esta fuente de interferencia.

Las mejores condiciones de medida, independientemente del valor de $Z_{\mathrm{C}}$, son: una alta capacidad paciente-tierra $\left(C_{\mathrm{B}}\right)$, un bajo desbalance entre las impedancias de electrodo $\Delta Z_{\mathrm{E}}$ y altos valores de $Z_{\mathrm{ISO}}$ (impedancia de aislación) así también como de CMRR. Todo esto contribuye a reducir $Z_{\mathrm{CE}}$, pero el valor óptimo de $Z_{\mathrm{C}}$ no es obvio a partir de la expresión analítica de $Z_{\mathrm{CE}}$.

El análisis de $Z_{\mathrm{CE}}$ como una función de la variable compleja $Z_{\mathrm{C}}$ muestra que el módulo mínimo de $Z_{\mathrm{CE}}$ se produce para valores extremos de $Z_{\mathrm{C}}$ (esto es $Z_{\mathrm{C}}$ nula o infinita). Es posible definir un valor de desbalance crítico $\Delta Z_{\mathrm{E} \text {.Crítico }}$ que es función del CMRR del A.B., de $C_{\mathrm{B}}$ y de $Z_{\mathrm{ISO}}$. Este parámetro determina cual de las alternativas es la más adecuada para un caso particular. Los resultados del análisis previenen que valores elevados, tanto resistivos como inductivos de $Z_{\mathrm{C}}$, deben evitarse porque pueden conducir a imprevistamente altos niveles de interferencia.

Por otra parte, en las condiciones típicas de medida, la dependencia de $Z_{\mathrm{CE}}$ con $Z_{\mathrm{C}}$ sólo es significativa para valores tan elevados de $Z_{\mathrm{C}}$ (centenas de $M \Omega$ ), que resultan difíciles de implementar ${ }^{5}$. Se podría decir, que usualmente, una alta $Z_{\mathrm{C}}$ o una baja $Z_{\mathrm{C}}$, ambas conducen a similares niveles de rechazo de la interferencia de red. Una $Z_{\mathrm{C}}$ baja tiene la ventaja de fijar reducidas tensiones de modo común, admitiendo mayores corrientes $i_{\mathrm{P}}$ de acoplamiento al paciente y convirtiéndose con esto en una alternativa atractiva y actual para mediciones de dos electrodos.

\footnotetext{
${ }^{5}$ Recordar que esta $Z_{\mathrm{C}}$ se define a $50 \mathrm{~Hz}$. La capacidad de los cables blindados, la capacidad de entrada propia de los A.B., así también como las eventuales protecciones colocadas a la entrada del mismo, hacen que sea difícil conseguir valores muy elevados de $Z_{\mathrm{C}}$ a $50 \mathrm{~Hz}$.
} 
Capítulo 3 - Interferencia de la tensión de red en sistemas de dos electrodos 


\section{Capítulo 4: Determinación Experimental del Modelo de EMI}

\section{Resumen}

Para evaluar el comportamiento de un A.B., desde el punto de vista de su rechazo a EMI de la tensión de red, es necesario conocer las exigencias a las que está sometido. Para ello es necesario conocer el valor de los distintos elementos que componen el modelo de EMI.

Por otra parte, el conocimiento, al menos aproximado de estos elementos, resulta útil para definir las especificaciones de diseño de un sistema de adquisición de biopotenciales y para estimar las relaciones Señal/Ruido (SNR) esperables.

Los parámetros de mayor importancia son las capacidades vinculadas al paciente: $C_{\mathrm{P}} \mathrm{y}$ $C_{\mathrm{B}}$, las asociadas al amplificador: $C_{\mathrm{SUP}}$ y $C_{\mathrm{ISO}}$, las impedancias de electrodo $Z_{\mathrm{E} 1}, Z_{\mathrm{E} 2}$ y $Z_{\mathrm{E} 3} \mathrm{y}$ en menor grado aquellas asociadas a los cables de conexión: $C_{\mathrm{SH} 1}, C_{\mathrm{SH} 2}, C_{\mathrm{L} 1} \mathrm{y}$ $C_{\mathrm{L} 2}$. En la literatura se reportan valores típicos de estos elementos, pero en muchos casos no son representativos la situación real de un amplificador particular en ensayo.

Se diseñaron técnicas de medida específicas para determinar el valor de los parámetros del modelo de EMI, intentando en lo posible estimarlos en condiciones reales de funcionamiento. En este capítulo se presentan los métodos de medida desarrollados y resultados experimentales ${ }^{1}$, los cuales permitieron verificar el modelo de EMI propuesto en los capítulos previos.

\footnotetext{
${ }^{1}$ Se incluyen solo algunos resultados experimentales a fin de validar los métodos propuestos. La idea no es generar datos estadísticos sino proporcionar métodos sencillos que permitan estimar rápidamente lo parámetros para condiciones particulares de medida.
} 


\section{Estimación de los elementos del modelo de EMI.}

Como se mostró en CAPítulo 2, los efectos dominantes en cuanto a la interferencia de la tensión de la red son: la tensión de modo diferencial debida a las corrientes de desplazamiento $i_{\mathrm{L}}$ acopladas a los cables de conexión y la transformación de la tensión de modo común $V_{\mathrm{CM}}$ en tensión de modo diferencial producida en forma externa al A.B. Para analizar estos dos mecanismos, el modelo de EMI puede reducirse al esquema que se muestra en la Fig. 4.1

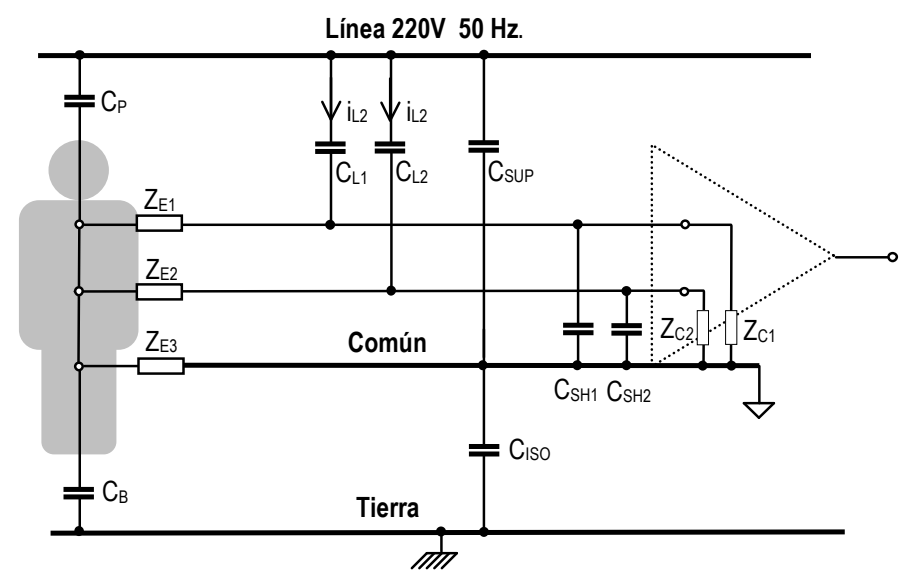

Figura 4.1. Modelo de EMI simplificado que contempla los elementos dominantes.

Algunos de los elementos que componen este esquema, como las capacidades propias de los cables blindados, la impedancia de aislamiento $C_{\mathrm{ISO}}$ e incluso la capacidad paciente-tierra $C_{\mathrm{B}}$, pueden medirse utilizando técnicas estándar, por ejemplo con un puente de impedancias o un multímetro con capacímetro.

Por otra parte, las impedancias vinculadas a la línea de $220 \mathrm{~V}$, así también como las impedancias de los electrodos, requieren de técnicas especialmente diseñadas. A continuación se proponen algunas estrategias para determinar el valor de estos elementos.

\subsection{Determinación de $C_{B}$ y $C_{P}$.}

Estas capacidades son los principales elementos del modelo y forman parte aún de los modelos más simples de EMI para aplicaciones biomédicas. La determinación $C_{\mathrm{B}}$ y $C_{\mathrm{P}}$ es especialmente importante porque junto con $C_{\text {ISO }}$ y $C_{\text {SUP }}$, definen las tensiones de modo común y modo aislante a las que estará sometido el A.B. (ver APÉNDICE 1).

Se han propuesto varios métodos para la estimación de estas capacidades. Las técnicas generalmente se basan en medir la tensión paciente-tierra $V_{\mathrm{P} 0}$ conectando impedancias conocidas, usualmente un resistor $R_{\mathrm{L}}$, entre paciente y tierra (ver Fig.4.2). Como se mostrará a continuación, el potencial del paciente resulta función de las capacidades $C_{\mathrm{P}}, C_{\mathrm{B}}$ y del valor de $R_{\mathrm{L}}$ utilizado en cada una de las medidas. A partir de dos medidas 
de $V_{\mathrm{P} 0}$ efectuadas con distintas $R_{\mathrm{L}}$ es posible estimar los valores de $C_{\mathrm{P}}$ y $C_{\mathrm{B}}$. (CRUMP $\&$ COSENTINO, 1973) (PALLAS-ARENY \& COLOMINAS, 1989).

El método que se propone en esta tesis, puede considerarse una extensión del propuesto por (CRUMP \& COSENTINO, 1973). Su principal ventaja es que, bajo ciertas condiciones, también puede utilizarse para estimar $C_{\mathrm{SUP}}$ y $C_{\mathrm{ISO}}$.

La técnica consiste en conectar un único electrodo al paciente mediante uno de los cables de conexión del equipo e insertar entre este cable y tierra un resistor variable $R_{\mathrm{L}}$. Esto corresponde al esquema ${ }^{2}$ de la Fig.4.2 con la llave en la posición " $\mathrm{A}$ ".

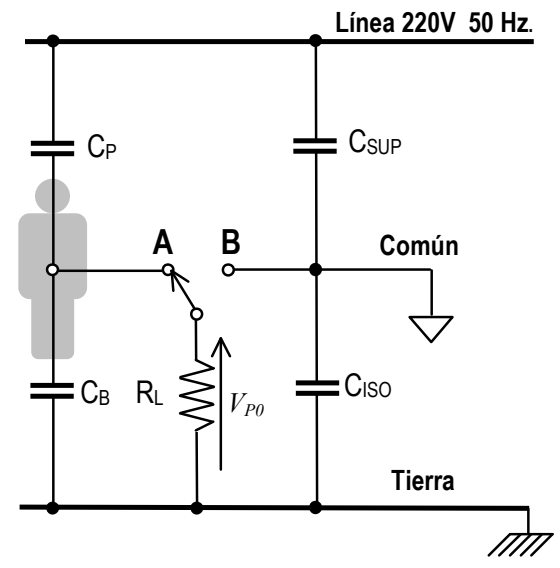

Figura 4.2. Esquema del circuito necesario para implementar el método propuesto.

En estas condiciones, el valor eficaz de la tensión $V_{\mathrm{P} 0}$, medida sobre el resistor $R_{\mathrm{L}}$, está dada por:

$$
V_{\mathrm{PO}}=\frac{220 \mathrm{~V} \cdot 2 \pi 50 \mathrm{~Hz} \cdot C_{\mathrm{P}} R_{\mathrm{L}}}{\sqrt{1+\left(2 \pi 50 \mathrm{~Hz}\left(C_{\mathrm{B}}+C_{\mathrm{P}}\right) R_{\mathrm{L}}\right)^{2}}}
$$

Graficando $V_{\mathrm{PO}}$ como función de $R_{\mathrm{L}}$ en forma logarítmica, es decir confeccionando un diagrama de Bode con $R_{\mathrm{L}}$ como variable independiente, resulta una curva como la indicada en la Fig.4.3. Sus asíntotas corresponden a:

$$
\begin{aligned}
& \text { si } R_{\mathrm{L}} \rightarrow 0 \quad\left|V_{\mathrm{PO}}\right| \cong 220 \mathrm{~V} \cdot 2 \pi 50 \cdot C_{\mathrm{P}} R_{\mathrm{L}} \\
& \text { si } R_{\mathrm{L}} \rightarrow \infty \quad\left|V_{\mathrm{PO}}\right| \cong 220 \mathrm{~V} \frac{C_{\mathrm{P}}}{C_{\mathrm{P}}+C_{\mathrm{B}}},
\end{aligned}
$$

\footnotetext{
${ }^{2}$ En este circuito equivalente se despreció la impedancia del electrodo, dado que se considera mucho menor que $R_{\mathrm{L}}$.
} 
Para valores pequeños de $R_{\mathrm{L}}$, la asíntota es una recta de $20 \mathrm{~dB}$ por década. Para valores elevados de esta variable la tensión $V_{\mathrm{PO}}$ se vuelve constante. El valor de $R_{\mathrm{L}}$ correspondiente al "codo" está dado por:

$$
R_{\mathrm{L} . \text { Codo }}=\frac{1}{2 \pi 50 \mathrm{~Hz}\left(C_{\mathrm{P}}+C_{\mathrm{B}}\right)}
$$

Como puede observarse en (4.2), la pendiente ${ }^{3}$ de la curva para $R_{\mathrm{L}}$ "pequeños", permite determinar $C_{\mathrm{P}}$. A partir del valor que adopta $V_{\mathrm{PO}}$ para elevadas $R_{\mathrm{L}}$ y utilizando el $C_{\mathrm{P}}$ obtenido previamente, se determina finalmente $C_{\mathrm{B}}$. De este modo, las capacidades $C_{\mathrm{P}}, C_{\mathrm{B}}$ pueden estimarse en forma simple a partir de las asíntotas dadas por (4.2) y (4.3), las cuales pueden obtenerse gráficamente o, disponiendo de capacidad de cómputo, a partir de un ajuste entre los datos experimentales y la expresión (4.1).

Es importante notar que este método permite estimar la capacidad $C_{\mathrm{B}}$ sólo si se dispone de medidas de $V_{\mathrm{P} 0}$ correspondientes a valores de $R_{\mathrm{L}}$ superiores a $R_{\mathrm{L} \text {.Codo }}$. De lo contrario, la curva obtenida no contendrá información sobre $C_{\mathrm{B}}$ sino únicamente sobre $C_{\mathrm{P}}$ (los valores de $R_{\mathrm{L}}$ no serían lo suficientemente altos para observar el codo de la curva). Considerando que la capacidad $C_{\mathrm{B}}$ adopta normalmente valores mayores a $100 \mathrm{pF}$, resultan $R_{\mathrm{L} \text {.Codo }}$ de aproximadamente $30 \mathrm{M} \Omega$; es decir que una $R_{\mathrm{L}}$ de algunos cientos de $\mathrm{M} \Omega$, será suficiente para obtener buenas medidas de $C_{\mathrm{B}}$.

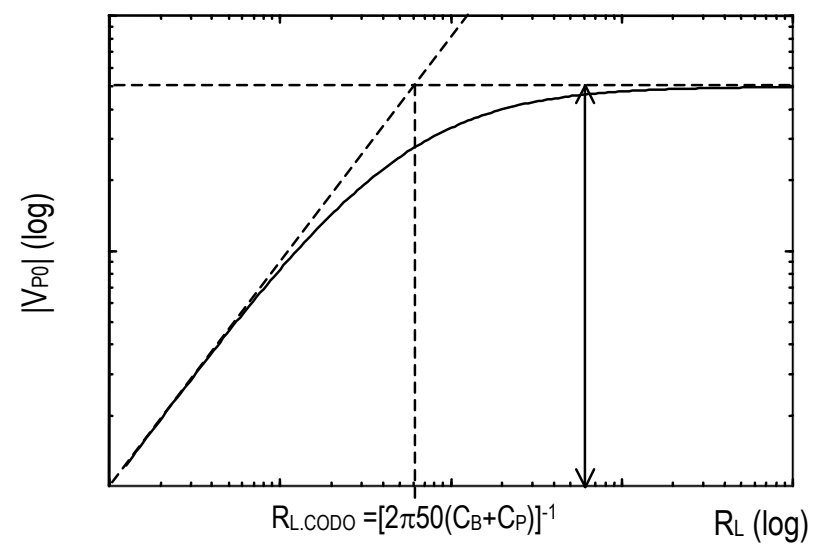

Figura 4.3. Tensión $V_{P 0}$ (Paciente -Tierra) como función del valor del resistor RL.

\subsection{Determinación de $\mathrm{C}_{\text {Iso }}$ y $\mathrm{C}_{\text {sup. }}$}

El mismo método expuesto en el punto anterior es aplicable para medir $C_{\mathrm{ISO}}$ y $C_{\mathrm{Sup}}$. La llave indicada en la Fig.4.2 debe colocarse en la posición "B" y la tensión $V_{\mathrm{P} 0}$ estará dada por:

$$
V_{\mathrm{PO}}=\frac{220 \mathrm{~V} \cdot 2 \pi 50 \cdot C_{\mathrm{SUP}} R_{\mathrm{L}}}{\sqrt{1+\left(2 \pi 50 \mathrm{~Hz}\left(C_{\mathrm{ISO}}+C_{\mathrm{SUP}}\right) R_{\mathrm{L}}\right)^{2}}} .
$$

\footnotetext{
${ }^{3} \mathrm{Si}$ el análisis se realiza a partir del gráfico log-log la pendiente de la asíndota de bajas $R_{\mathrm{L}}$ siempre será de $20 \mathrm{~dB}$ por década. El valor de $C_{\mathrm{P}}$ define el "desplazamiento" vertical de esta recta.
} 
Las asíntotas se transforman a:

$$
\begin{aligned}
& \text { Si } R_{\mathrm{L}} \rightarrow 0 \quad\left|V_{\mathrm{PO}}\right| \cong 220 \mathrm{~V} \cdot 2 \pi 50 \mathrm{~Hz} \cdot C_{\mathrm{SUP}} R_{\mathrm{L}} \\
& \text { Si } R_{\mathrm{L} \rightarrow \infty}\left|V_{\mathrm{PO}}\right| \cong 220 \mathrm{~V} \frac{C_{\mathrm{SUP}}}{C_{\mathrm{SUP}}+C_{\mathrm{ISO}}},
\end{aligned}
$$

y el valor de $R_{\mathrm{L}}$ correspondiente al "codo" queda dado por:

$$
R_{L . \text { Codo }}=\frac{1}{2 \pi 50 \mathrm{~Hz}\left(C_{\mathrm{SUP}}+C_{\mathrm{ISO}}\right)}
$$

En algunos casos, por ejemplo en pequeños amplificadores alimentados mediante baterías, el término $C_{\mathrm{SUP}}+C_{\mathrm{ISO}}$ puede ser de solo algunos $\mathrm{pF}$ y la capacidad $C_{\mathrm{ISO}}$ es difícil de determinar. Los valores de $R_{\mathrm{L} \text {.Codo }}$ resultan del orden de los $\mathrm{G} \Omega$ demandando valores de $R_{\mathrm{L}}$ tan altos que no pueden ser implementados.

\subsection{Resultados Experimentales.}

\subsubsection{Resistor Variable y Medidor de $V_{\mathrm{PO}}$.}

Para implementar el método descrito previamente, es necesario disponer de un resistor variable en un amplio rango de valores, digamos desde centenas de $\mathrm{k} \Omega$ hasta algún G $\Omega$. Resistores de tan elevado valor no son tecnológicamente simples de implementar. Por esto, se optó por simular el resistor mediante un circuito tipo bootstrap como el que se muestra en la Fig.4.4, el cual permite simular resistores de valor elevado utilizando resistores de valor moderado.

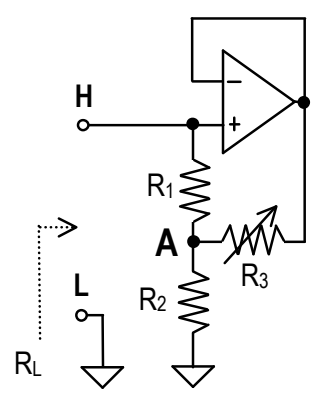

Figura 4.4. Circuito bootstrap utilizado para simular resistores de valor elevado.

Como se muestra en el análisis del APÉNDICE 4, dentro de ciertas limitaciones, impuestas por la tensión de offset y el ruido propio del A.O, la resistencia equivalente que este circuito presenta entre los terminales $\mathrm{H}$ y L, está dada aproximadamente por:

$$
R_{L} \cong R_{1} \cdot R_{2} / R_{3}
$$


Para simular un resistor $R_{\mathrm{L}}$ variable se adoptó como componente de ajuste $R_{3}$. Seleccionando distintos valores de $R_{3}$ mediante "jumpers", fue posible obtener valores de $R_{\mathrm{L}}$ entre $1 \mathrm{M} \Omega$ y $1 \mathrm{G} \Omega$. Para las medidas que requirieron resistores $R_{\mathrm{L}}$ menores de $1 \mathrm{M} \Omega$, se utilizaron resistores "reales".

\subsubsection{Capacidades propias del sistema de medida.}

En primer lugar, para verificar las posibilidades de medida del sistema implementado, se determinaron las capacidades propias del circuito respecto de la línea de distribución $\left(C_{\mathrm{P}}\right)$ y respecto de tierra $\left(C_{\mathrm{B}}\right)$. Para ello, simulando el gabinete de un instrumento biomédico, se montó el circuito en una caja metálica de aproximadamente $10 \times 10 \times 7 \mathrm{~cm}$ sin conectar ningún elemento al terminal " $H$ " tal como se muestra la Fig.4.5.

En estas condiciones, se relevó la tensión $V_{\mathrm{P} 0}$ en función de $R_{\mathrm{L}}$ obteniéndose la curva que se muestra en la Fig.4.6. Realizando un ajuste contra la expresión (4.1) se encontró que corresponde a capacidades ${ }^{4} C_{\mathrm{P} .0}=0.015 \mathrm{pF}$ y $C_{\mathrm{B} .0}=10 \mathrm{pF}$.

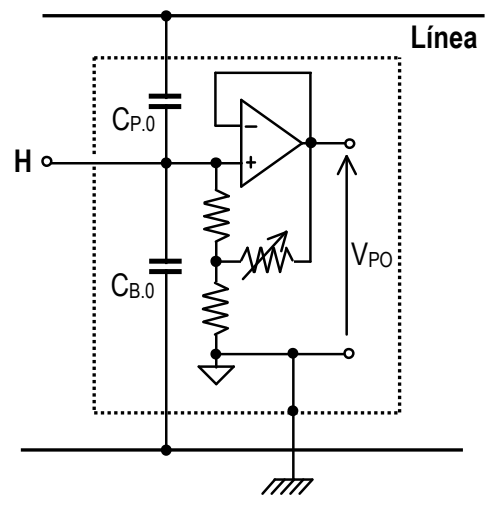

Figura 4.5. Circuito utilizado para determinar las capacidades propias del sistema de medida.

Estos valores determinan el alcance del método. Para que el mismo sea aplicable, las capacidades a medir deben ser al menos comparables a las propias del sistema de medida. Como se verá más adelante, el método es perfectamente aplicable a un entorno biomédico donde las capacidades referidas a la línea de distribución $\left(C_{\mathrm{P}}, C_{\mathrm{SUP}}\right)$ en general son de al menos décimas de $\mathrm{pF}$. Una situación límite puede producirse en la determinación de $C_{\mathrm{ISO}}$, porque considerando un amplificador pequeño, esta capacidad puede ser de solo algunos ${ }^{5} \mathrm{pF}$. En cuanto a la medición de $C_{\mathrm{B}}$, ésta no acarrea ningún problema porque usualmente su valor es de al menos alguna centena de $\mathrm{pF}$.

\footnotetext{
${ }^{4}$ Parte de la capacidad $C_{\mathrm{B}}$ medida es aportada por la capacidad de entrada $C_{\mathrm{IN}}$ del A.O utilizado (LF353) que posee una $C_{\mathrm{IN}}$ de unos $3-4 \mathrm{pF}$. El resto es atribuible a la capacidad entre el circuito, construido sobre un PCB de $4 \times 7 \mathrm{~cm}$, y tierra (gabinete).

${ }^{5}$ En este caso $C_{\text {ISO }}$ será detectable por el sistema de medida, aunque tendrá asociada un error importante.
} 


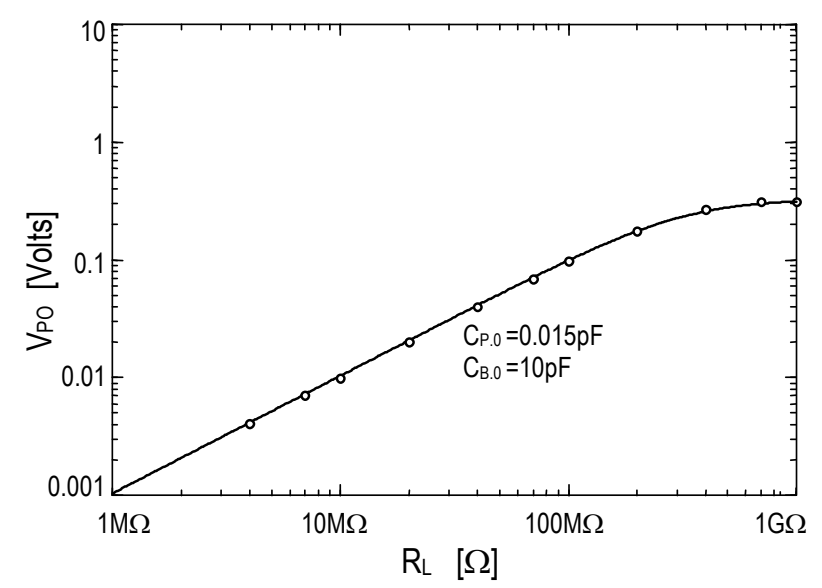

Figura 4.6. Datos experimentales correspondientes a las capacidades propias del sistema de medida. En línea llena se indica la curva de acuerdo a (4.5) correspondiente a $\mathrm{C}_{\mathrm{P} .0}=0.015 \mathrm{pF}$ y $\mathrm{C}_{\mathrm{B} .0}=10 \mathrm{pF}$.

\subsubsection{Capacidades al paciente.}

Para determinar estas capacidades se repitió el procedimiento descrito en el punto 2.1 utilizando la llave de la Fig.4.2 en la posición "A" con el paciente conectado. Los resultados experimentales, correspondientes a un paciente próximo a una mesa de trabajo (con un importante nivel de EMI) se muestran en la Fig.4.7. En este gráfico se consignan 3 medidas que corresponden a:

1. Paciente sentado en una silla aislada con sus pies en alto.

2. Paciente parado.

3. Paciente sentado en una silla aislada con sus pies apoyados en el piso.

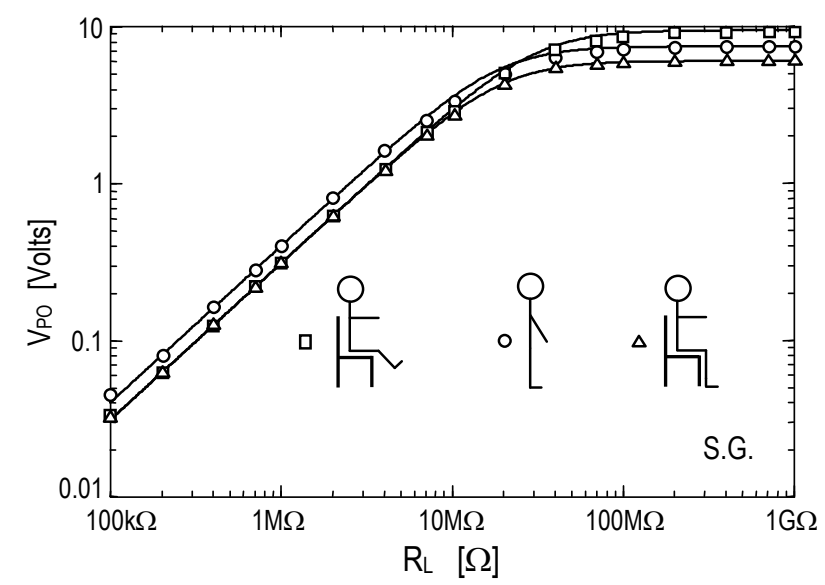

Figura 4.7. Determinación experimental de las capacidades $C_{P}$ y $C_{B}$. Las medidas experimentales indicadas con cuadrados corresponden al paciente sentado con pies en alto, las indicadas con círculos al paciente de pie y con triángulos al paciente sentado con pies en tierra. 
Se observa que con el paciente sentado con o sin sus pies apoyados en el piso, la asíntota de baja $R_{\mathrm{L}}$ (que depende de $C_{\mathrm{P}}$ ) es la misma, es decir que se mantiene el valor de $C_{\mathrm{P}}$. Por otra parte, el valor que adopta $V_{\mathrm{PO}}$ para altas $R_{\mathrm{L}}$ (asíntota de alta $R_{\mathrm{L}}$ ), es menor cuando los pies están apoyados en el piso. Esto muestra que al levantar los pies e incrementar la separación paciente-tierra, decrece el valor de $C_{\mathrm{B}}$.

Las medidas se efectuaron sobre dos pacientes y para cada caso se ajustaron los valores de $C_{\mathrm{P}}$ y $C_{\mathrm{B}}$ obteniéndose los resultados que se presentan en la Tabla I.

\begin{tabular}{llll}
\hline & \multicolumn{1}{c}{ Sentado. } & \multicolumn{1}{c}{ Sentado. } & Parado. \\
& Pies en tierra & Pies en aire & \\
\hline S.G & $\mathrm{C}_{\mathrm{P}}=4.5 \mathrm{pF}$ & $\mathrm{C}_{\mathrm{P}}=4.5 \mathrm{pF}$ & $\mathrm{C}_{\mathrm{P}}=5.8 \mathrm{pF}$ \\
& $\mathrm{C}_{\mathrm{B}}=160 \mathrm{pF}$ & $\mathrm{C}_{\mathrm{B}}=100 \mathrm{pF}$ & $\mathrm{C}_{\mathrm{B}}=165 \mathrm{pF}$ \\
\hline F.V & $\mathrm{C}_{\mathrm{P}}=3.0 \mathrm{pF}$ & $\mathrm{C}_{\mathrm{P}}=3.0 \mathrm{pF}$ & $\mathrm{C}_{\mathrm{P}}=3.8 \mathrm{pF}$ \\
& $\mathrm{C}_{\mathrm{B}}=255 \mathrm{pF}$ & $\mathrm{C}_{\mathrm{B}}=105 \mathrm{pF}$ & $\mathrm{C}_{\mathrm{B}}=265 \mathrm{pF}$ \\
\hline
\end{tabular}

TABLA I. Valores de $C_{P}$ y $C_{B}$ obtenidos en forma experimental para dos sujetos y para distintas condiciones de medida.

Ambos pacientes son de similar contextura, la diferencia apreciable en $C_{\mathrm{B}}$ para la condición "pies en tierra" es atribuible diferencias en el calzado.

\subsubsection{Capacidades propias del amplificador de biopotenciales.}

Para determinar las capacidades asociadas propiamente al amplificador, se utilizó el esquema de la Fig.4.2, pero esta vez con la llave en la posición "B". Los resultados experimentales, que se muestran en la Fig.4.8, permitieron determinar las capacidades $C_{\text {SUP }}$ y $C_{\text {ISO }}$ de un pequeño amplificador alimentado a batería (sin sus cables de conexión) que resultaron: $C_{\mathrm{SUP}}=0.12 \mathrm{pF}$ y $C_{\mathrm{ISO}}=13 \mathrm{pF}$. Como el valor de $C_{\mathrm{ISO}}$ es reducido, es necesario desafectar la capacidad $C_{\mathrm{B} .0}$ propia del sistema determinada en el punto 2.3.2, resultando $C_{\mathrm{SUP}}=0.12 \mathrm{pF}$ y $C_{\mathrm{ISO}}=3 \mathrm{pF}$.

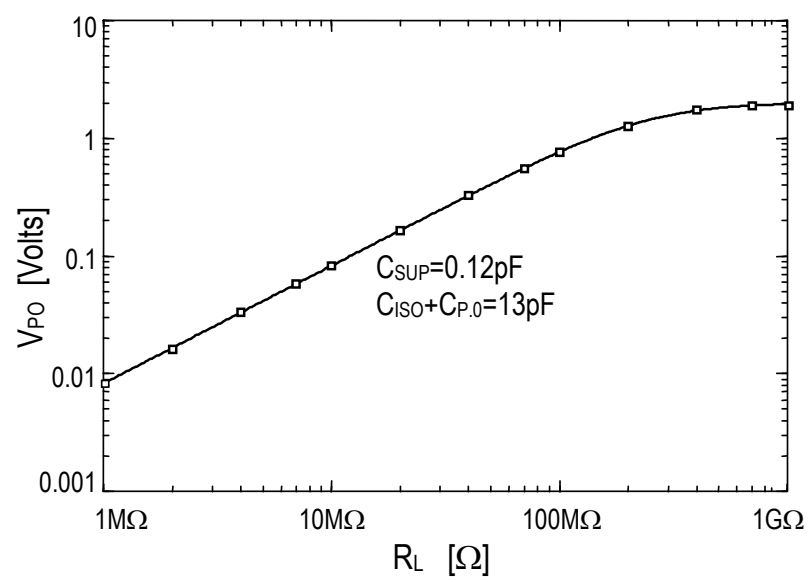

Figura 4.8.Datos experimentales correspondientes a las capacidades propias del amplificador Csup y Ciso. 


\subsection{Capacidades de acoplamiento a los cables de conexión.}

Si bien esta medida no tiene sentido práctico, pues utilizando cables blindados es muy fácil neutralizar los efectos de estas capacidades, se intentó igualmente determinarlas a fin de estimar la interferencia esperable en caso de utilizar cables sin blindaje.

Estas capacidades resultan simples de medir porque, como se analizó en el CAPÍTULO 2 , sus efectos son dominantes frente a los demás mecanismos de interferencia. Las corrientes $i_{\mathrm{L}}$ inducidas por la línea de $220 \mathrm{~V}$ a los cables, pueden determinarse utilizando el esquema que se muestra en la Fig.4.9. (HuHTA, 1973). Para su implementación solo se requiere de un osciloscopio.

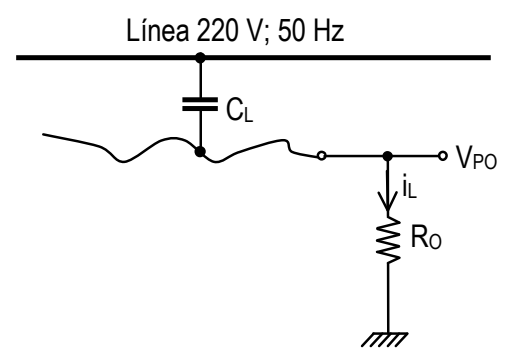

Figura 4.9. Esquema del método de medida de las capacidades a los cables de conexión.

En el circuito de la Fig.4.9, la tensión $V_{\mathrm{P} 0}$ está dada por:

$$
V_{\mathrm{PO}}=i_{\mathrm{L}} \cdot R_{\mathrm{O}}
$$

Entonces, midiendo $V_{\mathrm{PO}}$ y conociendo el valor del resistor $R_{\mathrm{O}}$, se determina $i_{\mathrm{L}}$ como:

$$
i_{\mathrm{L}}=\frac{V_{\mathrm{PO}}}{R_{\mathrm{O}}}
$$

Los efectos de este mecanismo de interferencia están directamente relacionados a esta corriente; si interesara conocer la capacidad $C_{\mathrm{L}}$, la misma puede estimarse a partir de $i_{\mathrm{L}}$ según:

$$
C_{\mathrm{L}} \cong \frac{i_{\mathrm{L}}}{2 \pi 50 \mathrm{~Hz} \cdot 220 \mathrm{~V}}
$$

\subsubsection{Resultados Experimentales.}

Para implementar este método, tal cual sugirieron Huhta y Webster (HUHTA \& WEBSTER,1973), como resistor $R_{\mathrm{O}}$ puede utilizarse directamente la impedancia de entrada propia del osciloscopio con el cual se efectúan la medidas de $V_{\mathrm{PO}}$. En general, esta impedancia de entrada es de $1 \mathrm{M} \Omega$ en paralelo con unos $20 \mathrm{pF}$ y a la frecuencia de red $(50 \mathrm{~Hz})$ puede considerarse resistiva pura. 
Con el fin de validar el método, se realizaron medidas utilizando distintos valores de $R_{\mathrm{O}}$. En la Fig.4.10 se muestra la tensión $V_{\mathrm{PO}}$ obtenida utilizando resistencias $R_{\mathrm{O}}$ de $100 \mathrm{k} \Omega, 430 \mathrm{k} \Omega, 1 \mathrm{M} \Omega$ y $3 \mathrm{M} \Omega$ sobre un cable sin blindaje de 1 metro de longitud.

Se realizaron dos ensayos: el primero apoyando el cable sobre una silla y el segundo manteniéndolo extendido y suspendido ${ }^{6}$. En el primer caso, del ajuste de los datos se obtiene una $i_{\mathrm{L}}=61 \mathrm{nA}\left(C_{\mathrm{L}} \approx 0.9 \mathrm{pF} / \mathrm{m}\right)$, mientras que en el segundo la corriente $i_{\mathrm{L}}$ resulta de $21 \mathrm{nA}\left(C_{\mathrm{L}} \approx 0.3 \mathrm{pF} / \mathrm{m}\right)$, es decir sensiblemente menor. Esto sugiere que, en caso de utilizar cables sin blindaje, es muy recomendable intentar que éstos no apoyen sobre ninguna superficie de área importante.

La dispersión entre muestras es baja (menor del 1\%) y por lo tanto una única medida es suficiente para determinar $i_{\mathrm{L}}$.

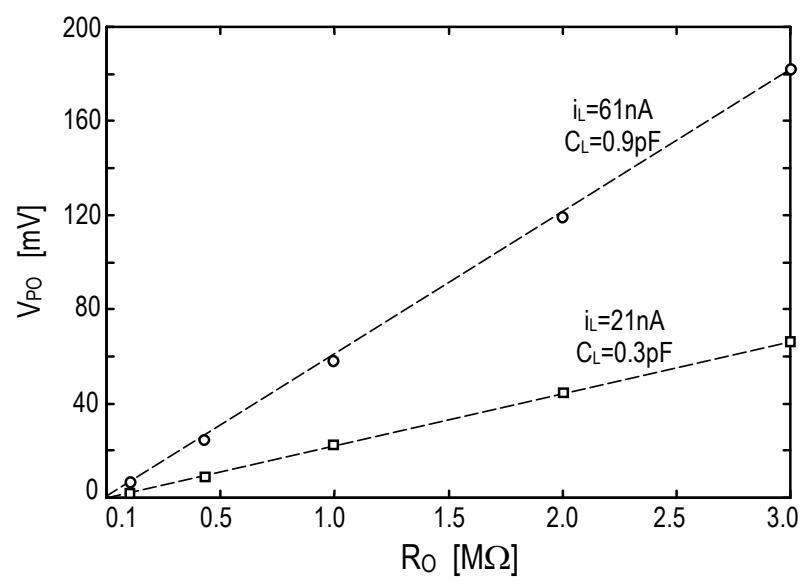

Figura 4.10. Determinación de la capacidad línea cable $C_{\llcorner}$para dos geometrías distintas.

\subsection{Medición de la capacidad propia de los cables de conexión.}

Las capacidades definidas entre el conductor central y la malla de los cables de conexión, denominadas $C_{\mathrm{SH} 1}$ y $C_{\mathrm{SH} 2}$ en el modelo de Fig.2.1, pueden medirse fácilmente con instrumentos estándar como un puente de impedancias o con un multímetro con medidor de capacidad. Se midieron cables blindados de uso común en audio con un puente RLC resultando capacidades entre 200 y $300 \mathrm{pF} / \mathrm{m}$.

\subsection{Medición del desbalance entre las impedancias de electrodo.}

Según se mostró en el CAPítulo 2, debido al efecto "divisor de potencial", el desbalance entre las impedancias de la interfase electro-piel degradan significativamente el rechazo a la tensión de línea. Este efecto produce una tensión diferencial de entrada dada por:

$$
V_{\mathrm{iD} . \mathrm{EMI}}=V_{\mathrm{CM}} \frac{\Delta Z_{\mathrm{E}}}{Z_{\mathrm{C}}}
$$

\footnotetext{
${ }^{6}$ Como si el cable fuera el hilo de una plomada de albañil.
} 
siendo:

$$
Z_{\mathrm{E}}=\frac{Z_{\mathrm{E} 1}+Z_{\mathrm{E} 2}}{2} ; \Delta Z_{\mathrm{E}}=Z_{\mathrm{E} 1}-Z_{\mathrm{E} 2}
$$

Como se observa en (4.13), es más importante conocer el desbalance $\Delta Z_{\mathrm{E}}$ que el valor de cada una de las impedancias.

Para evitar problemas producidos por la interferencia de la tensión de red, los equipos comerciales no miden las impedancias de los electrodos a $50 \mathrm{~Hz}$ sino a frecuencias por encima o por debajo de esta frecuencia. Usualmente se inyecta una corriente conocida y se mide la tensión que esta produce sobre la impedancia de electrodo. Esta técnica permite saber si algún contacto es defectuoso pero no permite conocer con precisión el desbalance $\Delta \mathrm{Z}_{\mathrm{E}}$ a $50 \mathrm{~Hz}$, por ejemplo para evaluar un nuevo diseño de un A.B.

Existe un ingenioso método propuesto por Grimbergen en (GRIMBERGEN, 1992) que utiliza la propia tensión de línea como fuente de corriente. Esta técnica permite medir cada una de las impedancias de electrodo por separado, pero la determinación del desbalance entre ellas implica realizar dos mediciones de impedancias (parte real y parte imaginaria) y calcular su diferencia. Esto, si bien es posible, es al menos engorroso.

En esta tesis se propone un nuevo método, basado en el propuesto por (GRIMBERGEN, 1992), que permite efectuar la medida del desbalance $\Delta Z_{\mathrm{E}}$ a la frecuencia de red en forma directa.

El método se basa en la aplicación de la ecuación (4.13). Si se consigue que la interferencia por "efecto divisor de potencial" sea dominante (esto es utilizar una $Z_{\mathrm{C}}$ relativamente baja, además de cables trenzados y blindados para evitar otros mecanismos de interferencia), la tensión de EMI $(50 \mathrm{~Hz})$ presente a la salida se corresponderá a la expresión (4.13). Es decir que será inversamente proporcional a $Z_{\mathrm{C}}$ y directamente proporcional a la tensión de modo común $V_{\mathrm{CM}} \mathrm{y}$ al desbalance $\Delta Z_{\mathrm{E}}$. Despejando $\Delta Z_{\mathrm{E}}$ de (4.13) se obtiene:

$$
\Delta Z_{E}=\frac{V_{\mathrm{iD.EMI}} \cdot Z_{C}}{V_{\mathrm{CM}}}
$$

Teniendo en cuenta que las variables involucradas en (4.15) son de tipo complejo, el módulo de $\Delta Z_{\mathrm{E}}$ resulta:

$$
\left|\Delta Z_{\mathrm{E}}\right|=\frac{\left|V_{\text {iD.EMI }}\right|\left|Z_{\mathrm{C}}\right|}{\left|V_{\mathrm{CM}}\right|}
$$

En esta expresión se observa que conociendo $Z_{\mathrm{C}}$, es posible estimar $\left|\Delta Z_{\mathrm{E}}\right|$ midiendo $\left|V_{\text {D.EMI }}\right|$ y $\left|V_{\mathrm{CM}}\right|$. El método también puede adaptarse para determinar parte real e imaginaria $^{7}$ de $\Delta Z_{\mathrm{E}}$, pero esto usualmente no es de gran interés y no se justifica la complejidad adicional ${ }^{8}$.

\footnotetext{
${ }^{7}$ Esto implica medir además la fase relativa entre $V_{\mathrm{iD} \cdot \mathrm{EMI}} \mathrm{y} V_{\mathrm{CM}}$.

${ }^{8}$ La medición de los módulos puede hacerse con un simple multímetro de mano, mientras que la medición de fase relativa requiere necesariamente de un osciloscopio.
} 
En la Fig. 4.10 se muestra el circuito utilizado para implementar la técnica propuesta. Este circuito incluye dos resistores $R_{\mathrm{C}}$ cuya función es asegurar una impedancia de modo común $Z_{\mathrm{C}}$ bien definida. Estos resistores deben ser de valor suficientemente elevados para que la aproximación lineal implícita en (4.10) sea aceptable (ver APÉNDICE 2) y no demasiado elevados para asegurar que el "efecto divisor de potencial" sea dominante. $R_{\mathrm{C}}=9.1 \mathrm{M} \Omega$ es un buen compromiso, al mismo tiempo que limita la corriente de falla al paciente (si este tocara Línea, es decir 220V) por debajo de los $50 \mu \mathrm{A}$ establecidos por la norma IEC601 ${ }^{9}$.

La tensión $V_{\text {D.EMI }}$ se obtiene mediante un amplificador de instrumentación, y la tensión de modo común partiendo el resistor $R_{\mathrm{G}}$ que define la ganancia del I.A. Para reducir ruido de alta frecuencia, se incluyeron filtros pasabajos para $V_{\mathrm{iD}}$ y $V_{\mathrm{CM}}$ con frecuencias de corte en $100 \mathrm{~Hz}$

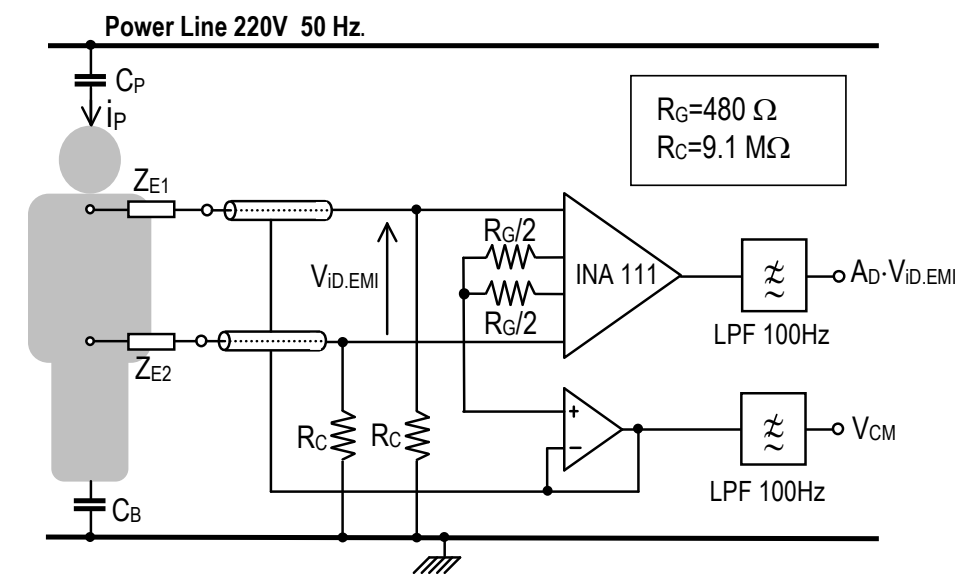

Figura 4.10.Esquema del sistema propuesto para medir desbalances en las impedancias electrodo-piel.

El circuito de la Fig.4.10 corresponde a un sistema de medida no-aislado. El método también puede aplicarse a sistemas aislados, pero en este caso las tensiones $V_{\mathrm{CM}}$ serán sensiblemente menores y se requiere amplificar también $V_{\mathrm{CM}}$. Además, deben utilizarse instrumentos flotantes.

Como puede advertirse en la Fig.4.10, un amplificador estándar puede fácilmente adaptarse para realizar esta medida. De hecho, para tomar las medidas que se muestran a continuación, se utilizó uno de los amplificadores desarrollados en esta tesis sin más modificación que incluir los resistores $R_{\mathrm{C}}$ a fin de degradar su impedancia $Z_{\mathrm{C}}$.

\subsubsection{Resultados Experimentales.}

Para validar el método propuesto se realizaron varios ensayos; algunos utilizando desbalances $\left|\Delta Z_{\mathrm{E}}\right|$ conocidos ("phantom") y otros sobre impedancias de electrodo reales.

\footnotetext{
${ }^{9}$ La normas de los EEUU son algo más estrictas y admiten solo hasta $10 \mu \mathrm{A}$. De todos modos, este circuito es para ser utilizado en experiencias de laboratorio y no para equipos médicos.
} 


\section{Medición de desbalances conocidos.}

Para esta prueba, se colocó un único electrodo sobre el paciente y se insertó un resistor en serie con una de las entradas del A.B. según se muestra en la Fig.4.11. De este modo, el desbalance $\left|\Delta Z_{\mathrm{E}}\right|$ está dado precisamente por el mencionado resistor.

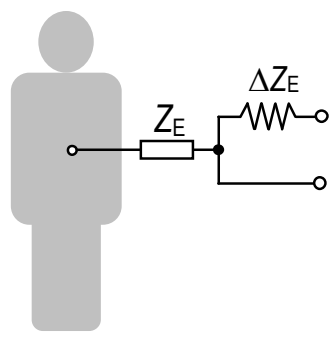

Figura 4.11. Esquema utilizado para simular desbalances $\Delta Z_{E}$ conocidos.

Se midieron la tensión de modo diferencial $\left(V_{\text {iD.EMI }}\right)$ y la de modo común $\left(V_{\mathrm{CM}}\right)$ utilizando como $\Delta Z_{\mathrm{E}}$ resistores de metal-film al $1 \%$ de $10 \mathrm{k} \Omega$ y $20 \mathrm{k} \Omega$. A fin de verificar la relación enunciada por (4.16), se efectuaron mediciones para varias $V_{\mathrm{CM}}$. Los distintos valores de $V_{\mathrm{CM}}$ se obtuvieron modificando la capacidad Línea-Paciente $C_{\mathrm{P}}$ tocando al paciente, poniéndolo en contacto con objetos de área importante (una mesa) y acercándolo a un conductor aislado conectado a la tensión de línea (220V).

Los resultados obtenidos se muestran en la Fig.4.12. Como puede observarse, los puntos experimentales siguen muy aproximadamente la relación lineal predicha por (4.16) (indicada en línea punteada).

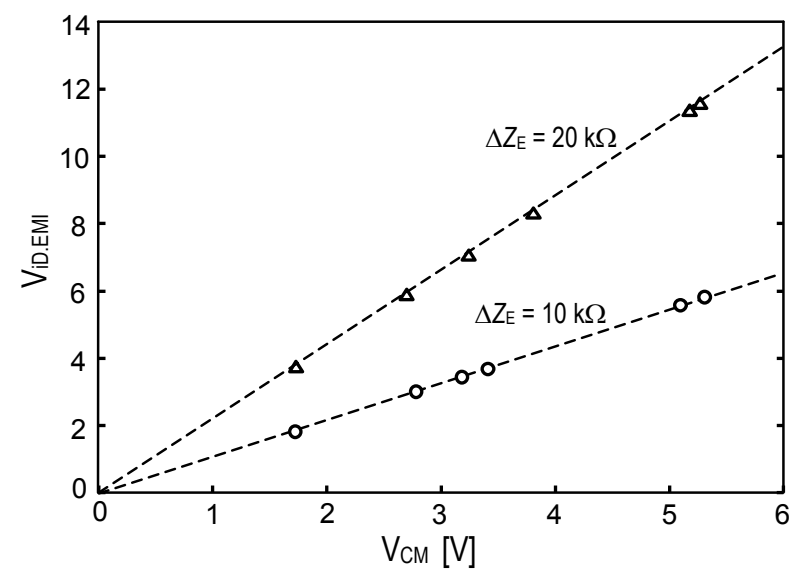

Figura 4.12. Relación entre tensiones de interferencia de modo común y modo diferencial para $\Delta Z_{\mathrm{E}}$ de $10 \mathrm{k} \Omega 20 \mathrm{k} \Omega$. Los datos experimentales están indicados con símbolos y las curvas teóricas en línea punteada.

\section{Medición de desbalances en impedancias electro-piel.}

Para medir $\Delta Z_{\mathrm{E}}$ reales, es decir entre impedancias electrodo-piel; en primer lugar se utilizaron dos electrodos tipo "placa" de $12 \mathrm{~cm}^{2}$ que fueron colocados en los brazos derecho e izquierdo (derivación I de ECG). Las medidas experimentales, las cuales se presentan en la Fig.4.13, determinaron un $\Delta \mathrm{Z}_{\mathrm{E}}$ de $4.4 \mathrm{k} \Omega$. 
Se efectuó un segundo ensayo reemplazando uno de los electrodos por uno de tipo "copa", de los utilizados habitualmente en ECG para las derivaciones precordiales. En este caso, el desbalance resultó de $36 \mathrm{k} \Omega$ : un valor significativamente mayor al caso previo donde ambos electrodos eran similares.

Todas las medidas fueron realizadas luego de 15 minutos de ser aplicados los electrodos. Para determinar $V_{\text {iD.EMI }}$ y $V_{\mathrm{CM}}$ se utilizó un osciloscopio digital (Tektronix TDS3012) en modo "promediación" disparado por línea. Esto permite eliminar la señal de ECG que podría influir en las medidas si la tensión $V_{\text {iD.EMI }}$ fuera muy baja. De todos modos, consiguiendo una $C_{\mathrm{P}}$ elevada (por ejemplo tocando al paciente) $V_{\text {iD.EMI }}$ es normalmente suficientemente alta como para permitir efectuar la medida con un simple multímetro.

En ambos ensayos, la desviación estandar entre medidas resultó de alrededor del $1 \%$. Esta baja dispersión implica que una única medida es suficiente para determinar $\Delta Z_{\mathrm{E}}$.

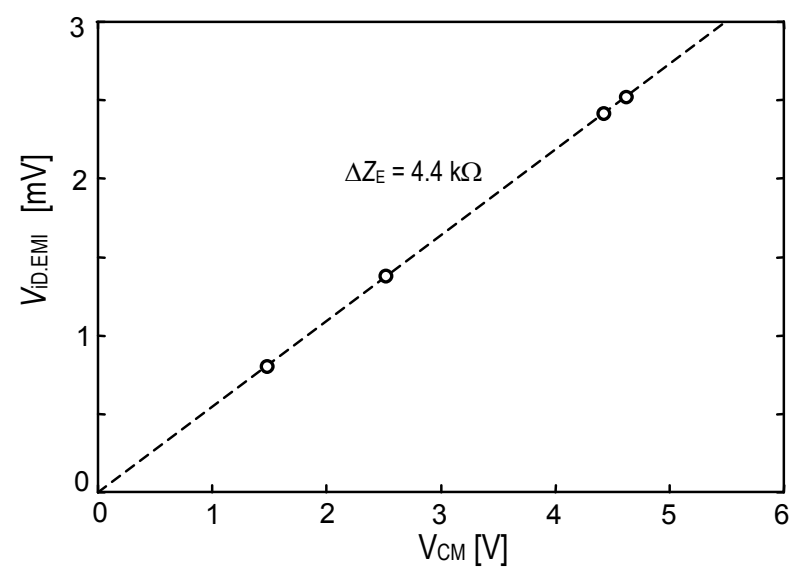

Fig. 4.13. Medidas experimentales para dos electrodos tipo "placa" de $12 \mathrm{~cm}^{2}$.

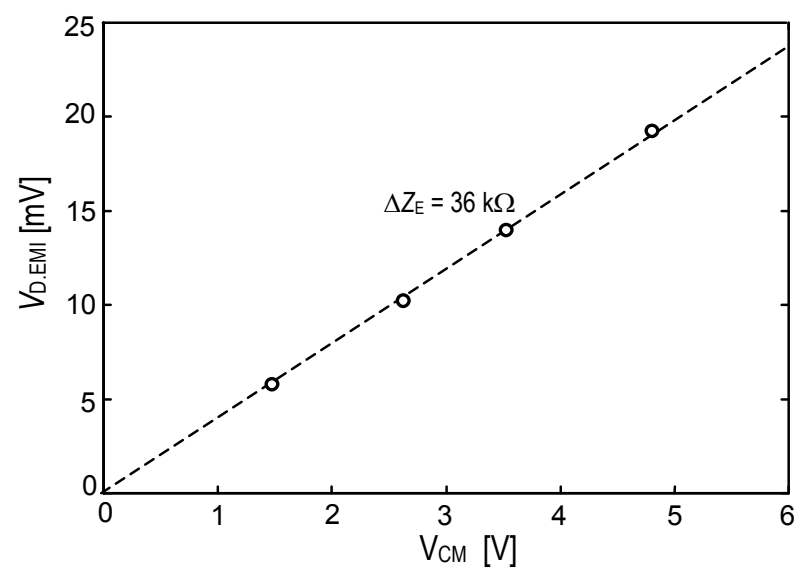

Figura 4.14. Medidas experimentales utilizando un electrodo tipo placa y uno tipo "copa". 


\subsection{Medición de las Impedancias de Entrada de Modo Común.}

Siguiendo un método similar al anterior, también es posible determinar la impedancia de modo común $Z_{\mathrm{C}}$ de un amplificador. El método propuesto se basa en el circuito que se muestra en la Fig.4.15. Un generador de modo común $V_{\mathrm{CM}}$ es aplicado a la entrada de un amplificador diferencial con un desbalance $\Delta Z_{\mathrm{E}}$ conocido entre sus entradas.

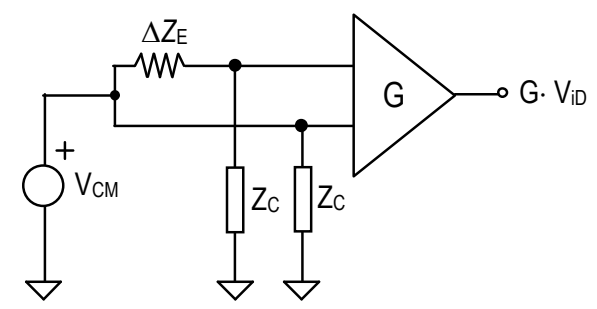

Figura 4.15. Esquema utilizado para determinar la impedancia de entrada para tensiones de modo común de un A.B.

De acuerdo al análisis presentado en el APÉNDICE 2, la tensión diferencial presente a la entrada del amplificador, estará dada por:

$$
V_{\mathrm{iD}}=V_{\mathrm{CM}} \frac{\Delta Z_{\mathrm{E}}}{Z_{\mathrm{C}}} \text {. }
$$

Despejando $\mathrm{Z}_{\mathrm{C}}$ resulta:

$$
Z_{\mathrm{C}}=V_{\mathrm{CM}} \frac{\Delta Z_{\mathrm{E}}}{V_{\mathrm{iD}}}
$$

Entonces, fijando $\Delta Z_{\mathrm{E}} \mathrm{y} V_{\mathrm{CM}}$, es posible estimar las impedancias $Z_{\mathrm{C}}$ midiendo $V_{\mathrm{iD}}$.

\subsubsection{Resultados Experimentales.}

Para conocer la dependencia de la impedancia de modo común $Z_{\mathrm{C}}$ de un A.B. con la frecuencia, se utilizó un generador de funciones para $V_{\mathrm{CM}}$, a fin de realizar un "barrido en frecuencia". Para cada una de las frecuencias utilizadas, se midió la tensión presente a la salida del amplificador, se la refirió a la entrada y se determinó la $Z_{\mathrm{C}}$ correspondiente a cada frecuencia mediante (4.18).

El resultado final de un ensayo sobre un amplificador tipo bootstrap que se analizará en el CAPítulo 6, se muestra en la Fig.4.16. Este amplificador permite neutralizar al menos parcialmente la capacidad de entrada de los A.O. y por eso el interés de determinar su $Z_{\mathrm{C}}$ con precisión. En general, es la capacidad de entrada de los A.O. quien limita la $Z_{\mathrm{C}}$ a algunos $\mathrm{pF}$, imponiendo un límite de tipo "tecnológico". En este caso, la $Z_{\mathrm{C}} @ 50 \mathrm{~Hz}$ resultó de $700 \mathrm{M} \Omega(800 \mathrm{M} \Omega$ en paralelo con $2.2 \mathrm{pF})$. 


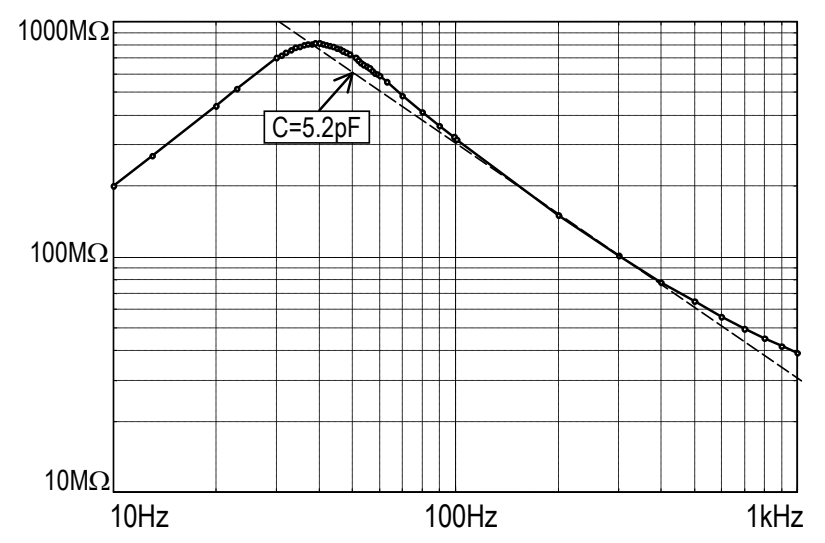

Figura 4.16. Impedancia de modo común de un A.B. determinada según el método propuesto. En línea puntada se indica la impedancia correspondiente a su capacidad de entrada $\left(\mathrm{C}_{\mathrm{IN}}=5.2 \mathrm{pF}\right)$.

\section{Conclusiones.}

Los elementos que componen el modelo EMI de un sistema de adquisición de biopotenciales pueden determinarse en forma experimental

Se presentaron métodos originales para la determinación de las capacidades $C_{\mathrm{P}}, C_{\mathrm{B}}$, $C_{\text {SUP }}$ y $C_{\mathrm{ISO}}$, para el desbalance $\Delta Z_{\mathrm{E}}$ entre las impedancias de electrodo y para la impedancia de entrada de modo común $Z_{\mathrm{C}}$.

Los métodos propuestos para la estimación de estos elementos, permiten hacerlo en condiciones de operación reales. Las experiencias realizadas permitieron validar el modelo de EMI introducido en los capítulos previos. 


\section{Capítulo 5: Topologías apropiadas para amplificadores de biopotenciales.}

\section{Resumen.}

En sistemas de tres electrodos, las principales condiciones que debe cumplir un A.B. son una alta impedancia de entrada $Z_{\mathrm{C}}$ para señales de modo común y un alto CMRR. En algunas aplicaciones, por ejemplo EEG o ECG de alta resolución, también es importante conseguir un muy bajo nivel de ruido.

Por otra parte, como se mostró en el CAPÍtUlo 3, los sistemas de dos electrodos presentan características distintas y distintivas y una baja $Z_{C}$ resulta ser conveniente.

Teniendo en cuenta estos aspectos, en este capítulo se analizan las topologías más apropiadas para implementar Amplificadores para Biopotenciales utilizando amplificadores operacionales.

La topología del A.B. también debe ser acorde a la configuración utilizada para la conexión de los electrodos. Se analizan aquí los casos más usuales que corresponden a las configuraciones "bipolar" y "monopolar". 


\section{Amplificadores Diferenciales.}

Un amplificador para biopotenciales, es básicamente un amplificador diferencial. Como las señales bioeléctricas se definen como diferencias de potencial entre dos electrodos, un A.B. debe tener necesariamente una entrada de tipo diferencial. Su salida puede ser también diferencial, resultando un amplificador F-D: FullyDifferential (entrada y salida diferenciales), o con salida respecto a masa, es decir S-E: Single-Ended. En la Fig.5.2. se muestran esquemas de ambos tipos de circuitos.
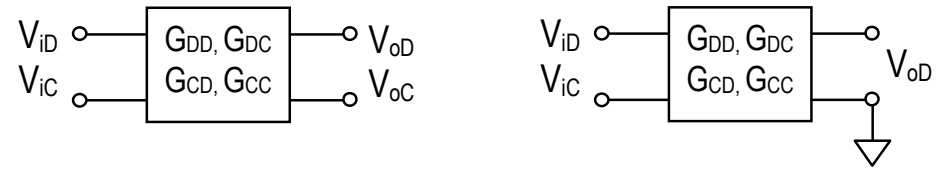

Figura 5.1. Tensiones de entrada y salida en una red Diferencial. (a) Circuito con entrada y salida diferenciales (F-D: Fully-Differential) y (b) Circuito con salida respecto de masa (S-E: Single-Ended).

En los circuitos F-D, tanto en su entrada como en su salida coexisten señales de modo común y de modo diferencial. Entre estas señales pueden definirse 4 funciones de trasferencia que conforman la denominada matriz de ganancias del amplificador. Los elementos de esta matriz se definen según:

$$
\begin{aligned}
& G_{\mathrm{DD}}=\left.\frac{V_{\mathrm{oD}}}{V_{\mathrm{iD}}}\right|_{V_{\mathrm{iC}}=0}=\frac{\text { salida } \mathrm{m} \cdot \text { diferencial }}{\text { entrada } \mathrm{m} \cdot \text { diferencial }} \\
& G_{\mathrm{DC}}=\left.\frac{V_{\mathrm{oD}}}{V_{\mathrm{iC}}}\right|_{V_{\mathrm{D}}=0}=\frac{\text { salida } \mathrm{m} \text {. diferencial }}{\text { entrada } \mathrm{m} \text {. común }} \\
& G_{\mathrm{CD}}=\left.\frac{V_{\mathrm{oC}}}{V_{\mathrm{iD}}}\right|_{V_{\mathrm{ic}}=0}=\frac{\text { salida } \mathrm{m} \text {. común }}{\text { entrada } \mathrm{m} \text {. diferencial }} \\
& G_{\mathrm{CC}}=\left.\frac{V_{\mathrm{oC}}}{V_{\mathrm{iC}}}\right|_{V_{\mathrm{iD}}=0}=\frac{\text { salida m. común }}{\text { entrada } \mathrm{m} \cdot \text { común }}
\end{aligned}
$$

Los elementos de mayor importancia son: la ganancia $G_{\mathrm{DD}}$ para señales de modo diferencial, la ganancia cruzada $G_{\mathrm{DC}}$ relacionada con las transformaciones de modo común a modo diferencial que ocurren dentro del amplificador, y $G_{\mathrm{CC}}$ que determina la propagación del modo común a través del amplificador.

En un amplificador S-E, uno de sus terminales de salida está fijo al potencial de masa y no tiene sentido su tensión de salida de modo común $V_{\mathrm{oC}}$, pues coincidiría con la de modo diferencial $V_{o D}$. En este caso solo se definen las ganancias $G_{\mathrm{DD}}$ y $G_{\mathrm{DC}}$. 
Existen varios factores de mérito para amplificadores diferenciales, el más importante es el Rechazo de Modo Común CMRR definido como:

$$
\mathrm{CMRR}=\frac{G_{\mathrm{DD}}}{G_{\mathrm{DC}}}
$$

\section{Configuraciones "bipolar" y "monopolar" en sistemas de tres electrodos.}

En un sistema de adquisición de biopotenciales multicanal, los electrodos pueden conectarse de distintas formas para definir las diferencias de potencial a capturar. Las configuraciones más usuales son la "bipolar" y la "monopolar".

El término "bipolar" se utiliza para denominar a aquellos sistemas que registran las diferencias de potencial entre dos electrodos tomados de a pares. Por otra parte, la configuración "monopolar" registra los potenciales de los distintos electrodos respecto de uno particular tomado como referencia (WEBSTER, 1992)(RolfE, 1983). Como puede advertirse, la denominación de estas configuraciones no está relacionada con la "polaridad" de las señales sino con la forma en que se definen las señales de interés.

En la Fig.5.2. se muestra el conexionado de un sistema de 4 canales de entrada para realizar registros de tipo bipolar (a) y uno de tipo monopolar (b). En EEG, la configuración bipolar proporciona una mejor resolución espacial, pues los electrodos correspondientes a cada canal pueden colocarse muy próximos entre sí, mientras que la monopolar presenta la ventaja de utilizar un menor número de electrodos. Cada uno de estos casos requiere un diseño específico del amplificador de biopotenciales.

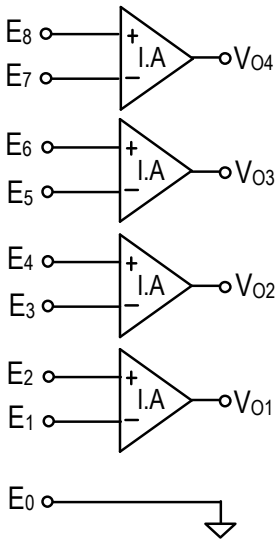

(a)

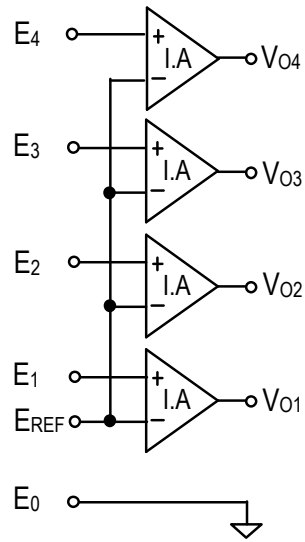

(b)

Figura 5.2. Configuración de electrodos (a) Bipolar y (b) Monopolar.

\subsection{Configuración Bipolar.}

En este caso el sistema de adquisición multicanal se forma por la simple adición de amplificadores independientes entre sí y el diseño de cada uno de ellos puede realizarse por separado. 
Existen varias topologías para implementar este tipo de amplificadores. Tal vez la más apropiada es el amplificador F-D de dos A.O que se muestra en la Fig.5.3. Este circuito presenta una alta $Z_{C}$, un bajo nivel de ruido y un alto CMRR sin requerir componentes apareados.

También existen alternativas con salida S-E, como el amplificador de instrumentación "clásico" de 3A.O.(Fig.5.4) o el de 2A.O. (Fig.5.5). Ambas configuraciones proveen una alta $Z_{C}$ y un bajo nivel de ruido, pero para conseguir altos CMRR, requieren asignar una alta ganancia al amplificador: algo que no siempre es posible en presencia de los potenciales de DC que se producen en la interfase electrodo-piel.

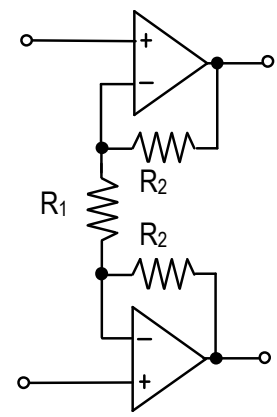

Figura 5.3. Amplificador de Instrumentación F-D.

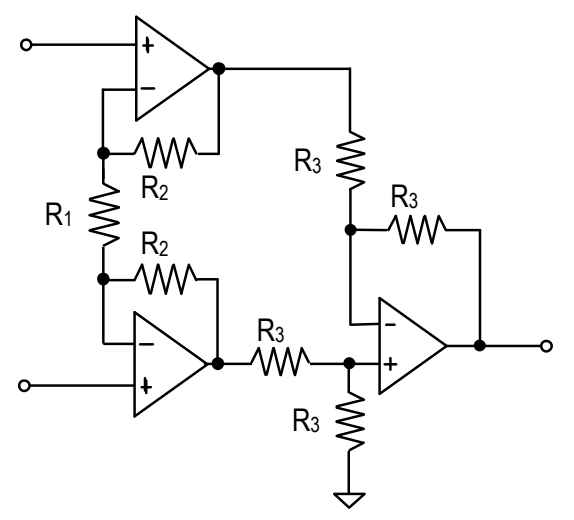

Figura 5.4. Amplificador de Instrumentación de 3 A.O.

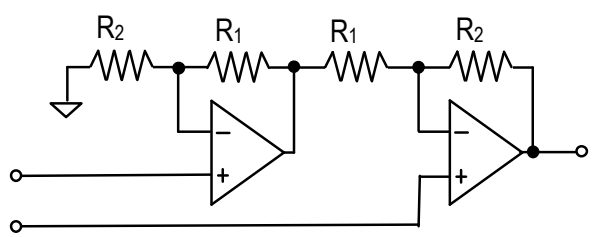

Figura 5.5. Amplificador de Instrumentación de 2 A.O.

A continuación, se presenta un análisis detallado de las principales características de cada una de estas topologías. 


\subsubsection{Amplificador Fully-Differential de 2 A.O.}

Este amplificador, que se muestra en la Fig.5.3, tiene tanto su entrada como su salida diferencial (F-D). Los circuitos F-D presentan varias ventajas:

1. Los rangos de entrada y de salida son el doble que los correspondientes a un circuito S-E. Por ejemplo, en un amplificador con salida S-E alimentado con $\pm V_{\mathrm{CC}}$, la amplitud máxima de salida es $V_{\mathrm{CC}}$, mientras que para uno con salida diferencial es de $2 V_{\mathrm{CC}}$ : un borne de salida puede tomar el potencial $+V_{\mathrm{CC}}$ y el otro $-V_{\mathrm{CC}}$. Este mejor aprovechamiento de la tensión de alimentación por parte de las estructuras F-D, las hace especialmente atractivas para la implementación de amplificadores alimentados a batería, donde la tensión de alimentación es un recurso escaso.

2. Los convertidores analógicos-digitales actuales de alta resolución generalmente poseen entrada diferencial. Si se utilizan circuitos F-D, los mismos pueden conectarse en forma directa al ADC sin requerir etapas de adaptación.

3. Tienen altos CMRR. Por ejemplo, asegurando que no existan conexiones a masa, su CMRR es idealmente infinito (ver APÉNDICE 5). Esto permite conseguir muy altos CMRR en amplificadores de varias etapas, siempre y cuando todas ellas sean F-D. Uno de los desafíos de esta configuración es conseguir una correcta propagación de la tensión de modo común sin incluir elementos a masa, es decir manteniendo una estructura perfectamente flotante.

4. Dado que tanto las señales de entrada como de salida se definen como diferencias de potencial entre dos nodos, el potencial de masa no tiene incidencia alguna. Esto relaja mucho el diseño del circuito impreso, dado que las diferencias del potencial de masa en distintas partes del circuito serán rechazadas como señales de modo común (ver Fig. 5.6).

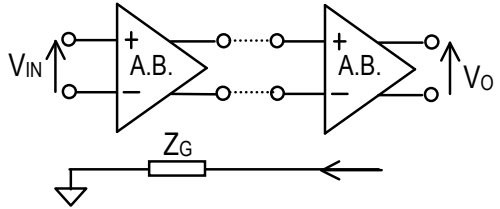

(a)

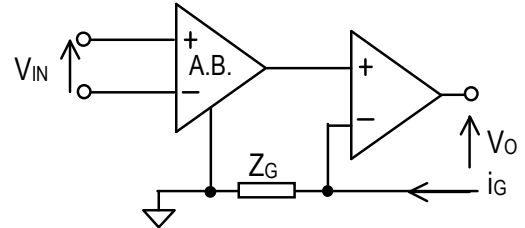

(b)

Figura 5.6. Interferencia por Impedancias Compartidas en la conexión de masa. (a) Circuitos F-D y (b) Circuitos S-E.

Una desventaja de las topologías F-D es que para implementar un circuito F-D se requieren más A.O. que para el caso de su contraparte S-E. (CASAS, 1996).

Los circuitos de tipo F-D son muy aconsejables y más aun en las primeras etapas. En el caso que el convertidor ADC posea una entrada de tipo S-E (hoy en día solo pequeños microcontroladores poseen este tipo de entrada ${ }^{1}$ ), Es conveniente realizar la conversión de diferencial a S-E en las últimas etapas donde el nivel de señal es significativo.

\footnotetext{
${ }^{1}$ Los convertidores ADC de alta resolución actuales (estamos hablando de 18-24 bits y del año 2004) son de tipo sigma-delta poseen casi exclusivamente entrada diferencial.
} 


\section{Funcionamiento del Circuito.}

A partir del circuito de la Fig.5.7, considerando A.O. ideales, se encuentra que la corriente sobre $R_{1}$ está dada por:

$$
i_{\mathrm{R} 1}=\frac{V_{\mathrm{iH}}-V_{\mathrm{iL}}}{R_{1}}
$$

Esta corriente $i_{\mathrm{R} 1}$, producirá una tensión diferencial de salida $V_{\mathrm{oD}}$ según:

$$
V_{\mathrm{oD}}=i_{\mathrm{R} 1} \cdot\left(R_{1}+R_{2}+R_{2}^{\prime}\right)
$$

Reemplazando (5.3) en (5.4) resulta:

$$
V_{\mathrm{oD}}=\left(V_{\mathrm{iH}}-V_{\mathrm{iL}}\right) \cdot \frac{R_{1}+R_{2}+R_{2}^{\prime}}{R_{1}} .
$$

si $R_{2} \cong R_{2}^{\prime},(5.5)$ puede escribirse como:

$$
V_{\mathrm{oD}}=V_{\mathrm{iD}} \cdot\left(1+2 R_{2} / R_{1}\right)
$$

De donde se obtiene que la ganancia $G_{\mathrm{DD}}$ de este circuito para señales de tipo diferencial está dada por:

$$
G_{\mathrm{DD}}=1+2 R_{2} / R_{1}
$$

A partir de (5.3) se observa que $i_{\mathrm{R} 1}$, que es la única corriente en el circuito, depende exclusivamente de la tensión diferencial de entrada y por lo tanto, no habrá corrientes relacionadas con la tensión de modo común $V_{\mathrm{iC}}$. Esto tiene varias consecuencias agradables: la ganancia $G_{\mathrm{DC}}$ (salida diferencial / entrada de modo común) será nula, la ganancia $G_{\mathrm{CC}}$ (salida modo común / entrada de modo común) será unitaria y el CMRR resultará idealmente infinito.

$$
G_{D C} \cong 0 \quad ; \quad G_{C C} \cong 1
$$

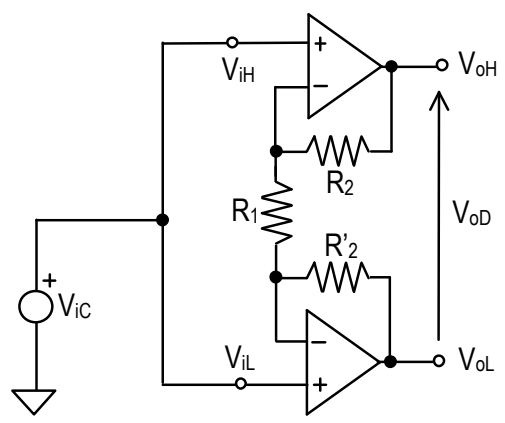

Figura 5.7. Amplificador F-D de dos A.O. 


\section{Rechazo de Modo Común.}

Si se considera que los A.O. tienen un determinado CMRR, la ganancia $G_{\mathrm{DC}}$ no será nula como se concluye a partir del modelo ideal utilizado en el punto previo, pero sí es muy baja, dando lugar a muy altos CMRR.

El CMRR de este circuito no depende de los desbalances que pudieran presentar los resistores, siendo esta una de sus principales ventajas. Como se muestra en el APÉNDICE 6, el rechazo de modo común depende exclusivamente de las diferencias entre el CMRR de cada uno de los A.O. que lo componen según:

$$
C M R R^{-1}=C_{\mathrm{AO} 2}^{-1}-C_{\mathrm{AO} 1}^{-1}
$$

Este circuito permite obtener elevados CMRR (de unos 110dB o más) aún en el caso de utilizar A.O. de propósitos generales.

\section{Ruido Equivalente a la Entrada.}

Esta topología también presenta muy buenas características en cuanto a su ruido propio. El ruido total referido a la entrada está determinado principalmente por el ruido de los A.O. que lo componen y es aproximadamente (APÉNDICE 6):

$$
e_{\mathrm{iT}}^{2} \cong 2 \cdot e_{\mathrm{AO}}^{2} \quad \text {. }
$$

Siendo $e_{\mathrm{iAO}}$ la tensión de ruido equivalente de un A.O.

\subsubsection{Amplificador de Instrumentación de 3 A.O.}

Este I.A. está compuesto por dos etapas. La primera de ellas consiste en un amplificador F-D de 2 A.O. y la segunda es un amplificador de diferencia que proporciona una salida S-E (Fig.5.8).

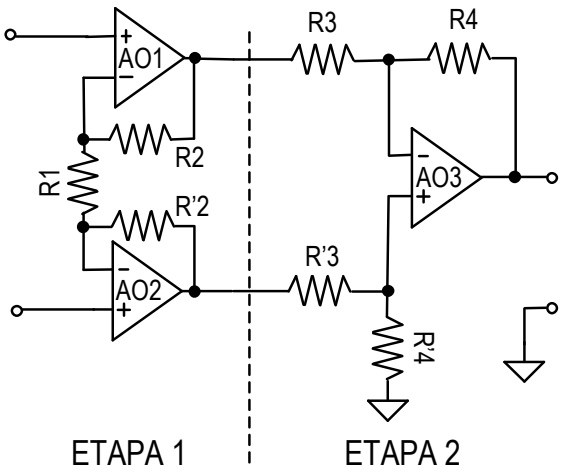

Figura 5.8. Amplificador de Instrumentación de tres A.O. 


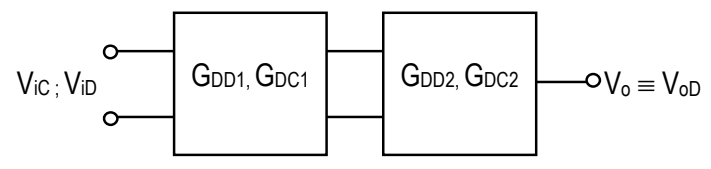

Figura 5.9. Esquema del Amplificador Multietapa de la Fig. 5.8. Cada unas de las etapas puede caracterizarse mediante su matriz de ganancias.

La ganancia de modo diferencial total del I.A. $G_{\mathrm{DD} . \mathrm{T}}$ está dada por el producto de las ganancias individuales de cada etapa:

$$
G_{\mathrm{DD} . \mathrm{T}}=G_{\mathrm{DD} 1} G_{\mathrm{DD} 2} \quad \text {. }
$$

Si se aplica una señal de entrada de modo común $V_{\mathrm{iC}}$, la tensión de salida $V_{\mathrm{o}}$ estará dada aproximadamente por:

$$
V_{\mathrm{oD}} \cong V_{\mathrm{iC}} \cdot G_{D C 1} G_{D D 2}+V_{\mathrm{iC}} \cdot G_{C C 1} G_{D C 2} .
$$

y la ganancia cruzada $G_{\mathrm{DC} . \mathrm{T}}$ del amplificador compuesto resulta:

$$
G_{\mathrm{DC} . \mathrm{T}}=G_{\mathrm{DC} 1} G_{\mathrm{DD} 2}+G_{\mathrm{CC} 1} G_{\mathrm{DC} 2}
$$

Para conseguir un elevado CMRR es deseable que $G_{\mathrm{DC} . \mathrm{T}}$, dada por (5.13), sea lo menor posible. Para su cálculo se requiere conocer $G_{\mathrm{DC} 1}$ y $G_{\mathrm{DC} 2}$. La ganancia $G_{\mathrm{DC} 2}$ de la segunda etapa (ver APÉNDICE 8) está dada por:

$$
G_{\mathrm{DC} 2}=\frac{4 \cdot t}{1+1 / G_{\mathrm{DD} 2}}+\frac{G_{\mathrm{DD} 2}}{C_{\mathrm{AO} 3}}
$$

siendo $t$ la tolerancia relativa de los resistores. La ganancia $\mathrm{G}_{\mathrm{DC} 1}$ correspondiente a la primer etapa (APÉNDICE 6) es:

$$
G_{\mathrm{DC} 1}=\left(1 / C_{\mathrm{AO} 2}-1 / C_{\mathrm{AO} 2}\right) G_{\mathrm{DD} 1}
$$

Reemplazando estas expresiones de $G_{\mathrm{DC} 2}(5.14)$ y $G_{\mathrm{DC} 1}(5.15)$ en (5.13) y teniendo en cuenta que $G_{\mathrm{CC} 1}$ es unitaria, finalmente se obtiene:

$$
G_{\mathrm{DC} . \mathrm{T}}=\left(1 / C_{\mathrm{AO} 2}-1 / C_{\mathrm{AO} 1}\right) G_{\mathrm{DD} . \mathrm{T}}+\left(\frac{4 \cdot t}{1+1 / G_{\mathrm{DD} 2}}+\frac{G_{\mathrm{DD} 2}}{C_{\mathrm{AO} 3}}\right) .
$$

El primer término depende solo de la ganancia total. En cuanto al segundo, para minimizar $G_{\text {DC.T }}$ se requiere una $\mathrm{G}_{\mathrm{DD} 2}$ tan baja como sea razonable, siendo usual adoptar $G_{\mathrm{DD} 2}=1$. Adoptando este último valor y teniendo en cuenta que el efecto dominante en $G_{\mathrm{DC} 2}$ es la tolerancia $t$ de los resistores (ver APÉNDICE 8), la ecuación (5.16) se transforma en:

$$
G_{\mathrm{DC} . \mathrm{T}}=\left(1 / C_{\mathrm{AO} 2}-1 / C_{\mathrm{AO} 1}\right) G_{\mathrm{DD} . \mathrm{T}}+2 \cdot t \quad,
$$


y el CMRR finalmente resulta:

$$
C M R R_{\mathrm{T}}^{-1}=\left(1 / C_{\mathrm{AO} 2}-1 / C_{\mathrm{AO} 1}\right)+\frac{2 \cdot t}{G_{\mathrm{DD} . \mathrm{T}}} \quad .
$$

A partir de (5.18) se observa que si la ganancia total $G_{\mathrm{DD} . \mathrm{T}}$ es elevada, el CMRR de esta configuración se vuelve independiente de la tolerancia de los resistores y se acerca al del amplificador F-D de dos A.O.

Como se comentó anteriormente, en un A.B. no es simple asignar una ganancia $G_{\mathrm{DD} . \mathrm{T}}$ elevada porque los potenciales de DC que se producen en la interfase electrodo-piel pueden llevar al amplificador a su saturación. El CAPítulo 6 de esta tesis se dedica especialmente a presentar soluciones a este problema particular de los amplificadores para biopotenciales.

\section{Ruido Equivalente a la entrada.}

Basta asignar una ganancia moderada a la primer etapa ( $>10$ veces) para que el ruido de este I.A quede dominado por los A.O. que componen la etapa de entrada. En estas condiciones, el ruido propio de este circuito es similar al del amplificador F-D de dos A.O. analizado en el punto previo.

\subsubsection{Amplificador de Instrumentación de 2. A.0.}

Utilizando 2 A.O. es posible construir un I.A de alta impedancia de entrada que se muestra en la Fig.5.10. Se trata de un amplificador diferencial de estructura desbalanceada; su CMRR en general no es muy elevado, pero la estructura es igualmente atractiva porque requiere un reducido número de componentes (MEETING VAN RIJN, 1994).

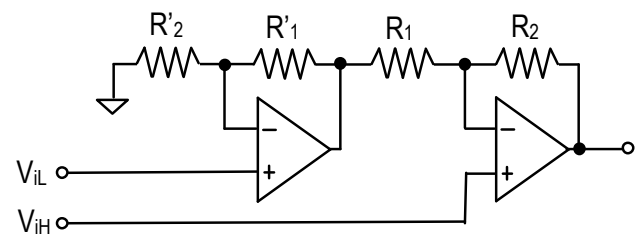

Figura 5.10. Amplificador de Instrumentación de 2. A.O.

\section{Funcionamiento del Circuito.}

La tensión de salida $V_{\mathrm{o}}$ de este circuito está dada por:

$$
\begin{aligned}
& V_{\mathrm{o}}=V_{\mathrm{iH}} \cdot\left(1+\frac{R_{2}}{R_{1}}\right)-V_{\mathrm{iL}} \cdot\left(1+\frac{R_{1}^{\prime}}{R_{2}^{\prime}}\right) \cdot\left(\frac{R_{2}}{R_{1}}\right) \\
& V_{\mathrm{o}}=V_{\mathrm{iH}} \cdot\left(1+\frac{R_{2}}{R_{1}}\right)-V_{\mathrm{iL}} \cdot\left(\frac{R_{1}^{\prime}}{R_{2}^{\prime}} \cdot \frac{R_{2}}{R_{1}}+\frac{R_{2}}{R_{1}}\right)
\end{aligned}
$$


Si se cumple,

$$
\frac{R_{2}}{R_{1}}=\frac{R_{2}^{\prime}}{R_{1}^{\prime}}
$$

el circuito se comporta como un amplificador diferencial y la tensión de salida resulta:

$$
V_{\mathrm{o}}=\left(1+\frac{R_{2}}{R_{1}}\right) \cdot\left(V_{\mathrm{iH}}-V_{\mathrm{iL}}\right)=G_{\mathrm{DD}} \cdot V_{\mathrm{iD}}
$$

Siendo $V_{\mathrm{iD}}=V_{\mathrm{iH}}-V_{\mathrm{iL}}$ la tensión de entrada de modo diferencial y $G_{\mathrm{DD}}=1+R_{2} / R_{1}$ la ganancia diferencial.

\section{Rechazo de Modo Común.}

Como se muestra en el APÉNDICE 9, el CMRR de este circuito está dominado por la tolerancia $t$ de los resistores y está dado aproximadamente por:

$$
\mathrm{CMRR} \cong \frac{G_{\mathrm{DD}}}{4 \cdot t}
$$

Se observa en (5.22), que para conseguir buenos CMRR es necesaria una ganancia $G_{\mathrm{DD}}$ elevada. Por otro lado, si se consigue asignar una alta $G_{\mathrm{DD}}$, se pondrán en evidencia otros problemas de este amplificador: los dos A.O trabajan en condiciones muy disímiles, uno de ellos tiene una fuerte realimentación mientras que el otro una muy débil, lo cual degrada el CMRR del circuito. Esta situación se agrava aún más al incrementarse la frecuencia y hacerse más evidentes los efectos de la ganancia finita de los A.O. (ver APÉNDICE 9). De todos modos, si la frecuencia de trabajo no es muy alta y no se requiere un CMRR muy elevado, esta configuración es útil y utilizada (MEETING VAN RIJN, 1994)

\section{Tensión Equivalente de Ruido a la entrada}

El ruido propio de este amplificador de instrumentación se debe fundamentalmente a los A.O. (APÉNDICE 9) y está dado aproximadamente por:

$$
e_{i T}^{2} \cong 2 e_{A O}^{2} \quad .
$$

\subsubsection{Amplificador de Instrumentación de 2 A.O. con ajuste de ganancia.}

El circuito de la Fig.5.10 presenta algunas dificultades. Para ajustar la ganancia $G_{\mathrm{DD}}$ es necesario modificar dos resistores al mismo tiempo y además este circuito no provee una estimación de la tensión de modo común de entrada $V_{\mathrm{iC}}$. Esto último es importante para implementar técnicas que hacen uso de la realimentación de la tensión de modo común. Estas estrategias se analizarán en el CAPÍTULO 6. 


\section{Ajuste de Ganancia.}

La ganancia del I.A de dos A.O. puede ajustarse incorporando un resistor adicional $\left(R_{\mathrm{G}}\right)$, entre las entradas inversoras de los A.O. según se muestra en la Fig.5.11.

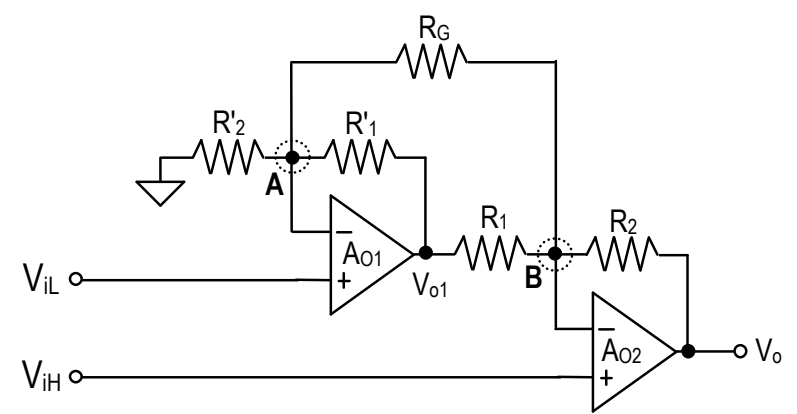

Figura 5.11. Amplificador de Instrumentación de 2 A.O. La inclusión de $R_{G}$ permite ajustar la ganancia mediante un único elemento.

El cálculo de la tensión de salida a partir de los potenciales $V_{\mathrm{iH}}, V_{\mathrm{iL}}$ en los bornes de entrada, es más complicado de lo que parece a simple vista. Una estrategia posible es planteando las ecuaciones de nodos en los puntos indicados como "A" y "B" en el esquema de la Fig.5.11.

1. Nodo A.

$$
\frac{V_{\mathrm{iH}}-V_{\mathrm{iL}}}{R_{\mathrm{G}}}+\frac{V_{\mathrm{o} 1}-V_{\mathrm{iL}}}{R_{1}^{\prime}}=\frac{V_{\mathrm{iL}}}{R_{2}^{\prime}}
$$

2. Nodo B.

$$
\frac{V_{\mathrm{o}}-V_{\mathrm{iH}}}{R_{2}}=\frac{V_{\mathrm{iH}}-V_{\mathrm{ol}}}{R_{1}}+\frac{V_{\mathrm{iH}}-V_{\mathrm{iL}}}{R_{\mathrm{G}}} .
$$

Despejando $V_{\mathrm{o} 1}$ de (5.24):

$$
V_{o 1}=\left(\frac{V_{\mathrm{iL}}}{R_{2}^{\prime}}+\frac{V_{\mathrm{iL}}-V_{\mathrm{iH}}}{R_{\mathrm{G}}}\right) \cdot R_{1}^{\prime}+V_{\mathrm{iL}},
$$

y despejando $V_{\mathrm{o}}$ de (5.25):

$$
V_{o}=\left(\frac{V_{i H}-V_{o 1}}{R_{1}}+\frac{V_{i H}-V_{i L}}{R_{G}}\right) \cdot R_{2}+V_{i H}
$$

Reemplazando (5.26) en (5.27)

$$
V_{\mathrm{o}}=\left(\frac{V_{\mathrm{iH}}}{R_{1}}-\frac{V_{\mathrm{iL}} \cdot R_{1}^{\prime}}{R_{2}^{\prime} \cdot R_{1}}-\frac{V_{\mathrm{iL}} \cdot R_{1}^{\prime}}{R_{\mathrm{G}} \cdot R_{1}}+\frac{V_{\mathrm{iH}} \cdot R_{1}^{\prime}}{R_{\mathrm{G}} \cdot R_{1}}-\frac{V_{\mathrm{iL}}}{R_{1}}+\frac{V_{\mathrm{iH}}}{R_{\mathrm{G}}}-\frac{V_{\mathrm{iL}}}{R_{\mathrm{G}}}\right) \cdot R_{2}+V_{\mathrm{iH}}
$$

y agrupando se obtiene:

$$
V_{\mathrm{o}}=\left(V_{\mathrm{iH}}-V_{\mathrm{iL}}\right) \frac{R_{2}}{R_{1}}+\left(V_{\mathrm{iH}}-V_{\mathrm{iL}}\right) \frac{R_{1}^{\prime} R_{2}}{R_{1} R_{\mathrm{G}}}+\left(V_{\mathrm{iH}}-V_{\mathrm{iL}}\right) \frac{R_{2}}{R_{\mathrm{G}}}+V_{\mathrm{iH}}-V_{\mathrm{iL}} \frac{R_{1}^{\prime} R_{2}}{R_{1} R_{2}^{\prime}} .
$$


Trabajando (5.29) puede llevarse a:

$$
V_{\mathrm{o}}=\left(V_{\mathrm{iH}}-V_{\mathrm{iL}}\right)\left(\frac{R_{2}}{R_{1}}+\frac{R_{1}^{\prime} R_{2}}{R_{1} R_{\mathrm{G}}}+\frac{R_{2}}{R_{\mathrm{G}}}\right)+V_{\mathrm{iH}}-V_{\mathrm{iL}} \frac{R_{1}^{\prime} R_{2}}{R_{1} R_{2}^{\prime}}
$$

Considerando resistencias perfectamente apareadas, es decir $R_{1}=R^{\prime}{ }_{1}, R_{2}=R^{\prime}{ }_{2},(5.30)$ se reduce a:

$$
V_{\mathrm{o}}=\left(V_{\mathrm{iH}}-V_{\mathrm{iL}}\right)\left(1+\frac{R_{2}}{R_{1}}+2 \frac{R_{2}}{R_{\mathrm{G}}}\right) .
$$

En estas condiciones, el circuito se comporta como un amplificador perfecto cuya ganancia $G_{\mathrm{DD}}$ está dada por:

$$
G_{\mathrm{DD}}=1+\frac{R_{2}}{R_{1}}+2 \frac{R_{2}}{R_{\mathrm{G}}}
$$

Como se demuestra en el APÉNDICE 9, tanto el CMRR como la tensión eficaz de ruido de esta configuración son similares a los correspondientes del I.A de dos A.O. Es decir que $R_{\mathrm{G}}$ afecta a la ganancia sin alterar significativamente el rechazo de modo común ni el nivel de ruido del amplificador.

\section{Estimación de la Tensión de Modo Común $V_{i c .}$}

Si se parte el resistor $R_{\mathrm{G}}$ en dos partes iguales, el punto medio común a estos dos nuevos resistores $R_{\mathrm{G}} / 2$ (ver Fig.5.12), adopta un potencial igual a la tensión de modo común de entrada $V_{\text {iC. }}$ De este modo se obtiene una salida de modo común, pero el ajuste de ganancia implica nuevamente, modificar dos resistores. Una forma de evitarlo es agregando un resistor $R_{\mathrm{G} 2}$ como muestra la Fig.5.12. En este caso, el nuevo resistor $R_{\mathrm{G}}$ equivalente corresponde al paralelo de $R_{\mathrm{G} 1}$ con $R_{\mathrm{G} 2}$. Si se desea mantener la relación original, entre la ganancia $G_{\mathrm{DD}}$ y $R_{\mathrm{G} 2}$ basta con tomar $R_{\mathrm{G} 1} \gg R_{\mathrm{G} 2}$.

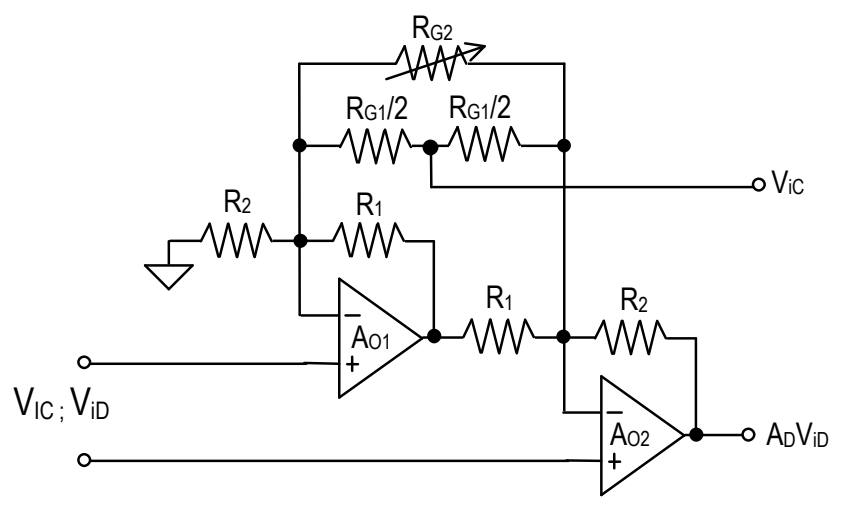

Figura 5.12. "Partiendo" el resistor $\mathrm{R}_{\mathrm{G}}$, es posible disponer de una estimación de la tensión de Modo Común de entrada $\mathrm{V}_{\mathrm{ic}}$. 


\subsection{Configuración Monopolar.}

Como se describió anteriormente, la configuración monopolar amplifica la tensión de cada electrodo respecto de uno particular definido como "referencia".

En principio podría utilizarse una batería de amplificadores bipolares según el esquema de la Fig.5.13, donde un electrodo es común a todos los canales, pero esta alternativa no es muy conveniente. Si se consideran N canales, la impedancia de entrada correspondiente al electrodo de referencia será el paralelo de $\mathrm{N}$ impedancias $Z_{\mathrm{C}}$, es decir $Z_{\mathrm{C} . \mathrm{REF}}=Z_{\mathrm{C}} / \mathrm{N}$, mientras que las entradas restantes presentan una impedancia de entrada $Z_{\mathrm{C}}$. Este desbalance, en presencia de impedancias de electrodo apenas moderadas, puede conducir a niveles de interferencia apreciables (efecto divisor de potencial).

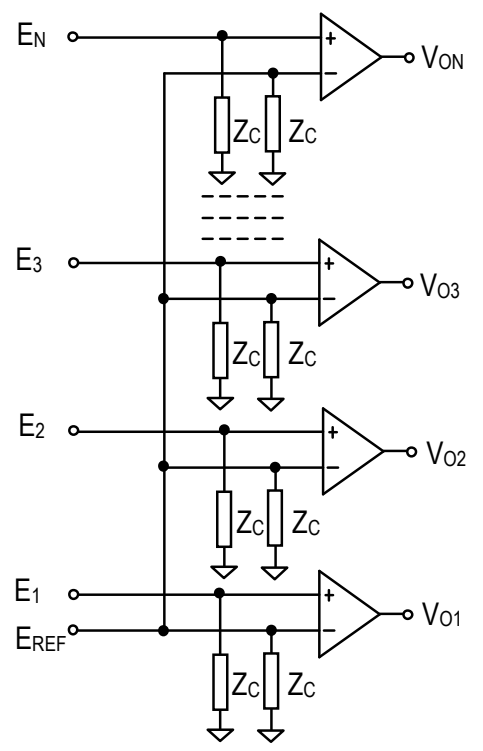

Figura 5.13. Amplificador Monopolar formado por $\mathrm{N}$ amplificadores bipolares con una entrada en común. La impedancia de entrada del electrodo de referencia resulta $\mathrm{N}$ veces menor que la correspondiente a los demás electrodos.

Por ejemplo, en un equipo de EEG de 20 canales, si se utilizaran amplificadores con $Z_{\mathrm{C}} @ 50 \mathrm{~Hz}=30 \mathrm{M} \Omega \quad\left(C_{\mathrm{IN}} \approx 100 \mathrm{pF}^{2}\right)$, el electrodo de referencia presentará una $Z_{\mathrm{C} . \mathrm{REF}}=1.5 \mathrm{M} \Omega$ mientras que las restantes entradas una $Z_{\mathrm{C}}=30 \mathrm{M} \Omega$. Si se consideran impedancias de electrodos perfectamente balanceadas $\left(Z_{\mathrm{E} 1}=Z_{\mathrm{E} 2}=Z_{\mathrm{E}}\right)$ la tensión de modo diferencial a la entrada estará dada (APÉNDICE 2) por:

$$
V_{\mathrm{oD}}=V_{\mathrm{iC}}\left(\frac{Z_{\mathrm{C} 1}}{Z_{\mathrm{C} 1}+Z_{\mathrm{E}}}-\frac{Z_{\mathrm{C} 2}}{Z_{\mathrm{C} 2}+Z_{\mathrm{E}}}\right) \text {. }
$$

\footnotetext{
${ }^{2}$ Esta $C_{\mathrm{IN}}$ incluye la capacidad propia de los cables de conexión.
} 
Para los valores analizados, considerando $Z_{\mathrm{E}}=10 \mathrm{k} \Omega$ y una $V_{\mathrm{iC}}=100 \mathrm{mV}$ resulta:

$$
V_{\mathrm{oD}}=100 \mathrm{mV}\left(\frac{30 \mathrm{M} \Omega}{30.01 \mathrm{M} \Omega}-\frac{1.5 \mathrm{M} \Omega}{1.51 \mathrm{M} \Omega}\right)=630 \mu \mathrm{V}
$$

Este nivel de interferencia no es aceptable y menos aún para EEG: una aplicación donde precisamente es habitual utilizar este número de electrodos y la configuración monopolar.

Una solución simple a este problema consiste en colocar un buffer de ganancia unitaria para cada uno de los electrodos o al menos para el electrodo de referencia (Fig.5.14) y luego sí proseguir con el conexionado monopolar.

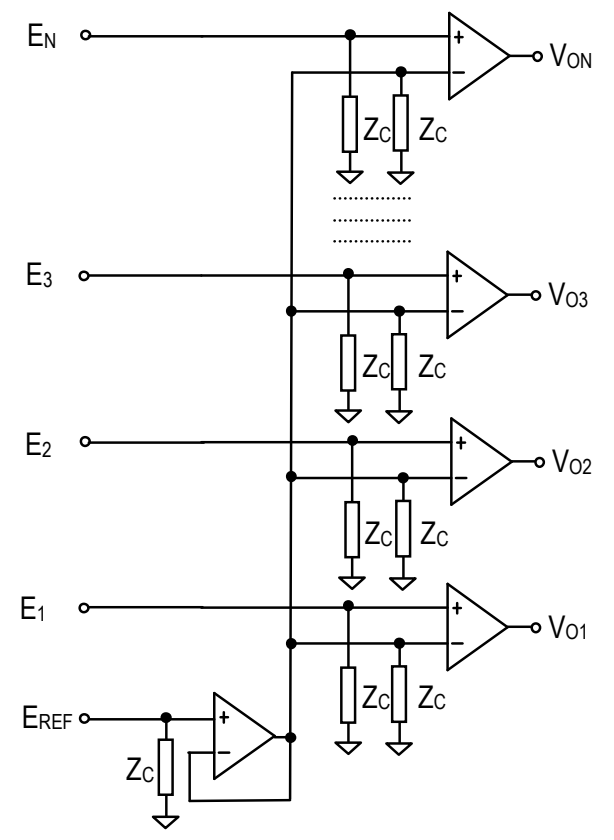

Figura 5.14. Amplificador Monopolar. Incluyendo un buffer en el canal de referencia todas las entradas presentan igual impedancia.

Este tipo de solución es simple y muy utilizada, pero no resulta del todo apropiada para aplicaciones de muy bajo ruido porque el seguidor de ganancia unitaria incrementa el ruido propio del A.B.

\subsubsection{Un Amplificador Monopolar propuesto.}

En esta tesis se propone un circuito alternativo para conseguir impedancias de modo común apareadas, sin incluir buffers de entrada. El circuito, de tipo F-D, se muestra en la Fig.5.15. Se arribó a esta configuración a partir de un circuito S-E presentado por (MEETING VAN RIJN, 1990.b). El circuito original presenta impedancias $Z_{\mathrm{C}}$ apareadas pero un CMRR moderado (90dB) y dependiente del desbalance entre los resistores. 
Como se mostrará a continuación, sin más modificaciones que tomar una salida de tipo diferencial, su CMRR se vuelve independiente de las tolerancias de componentes pasivos.

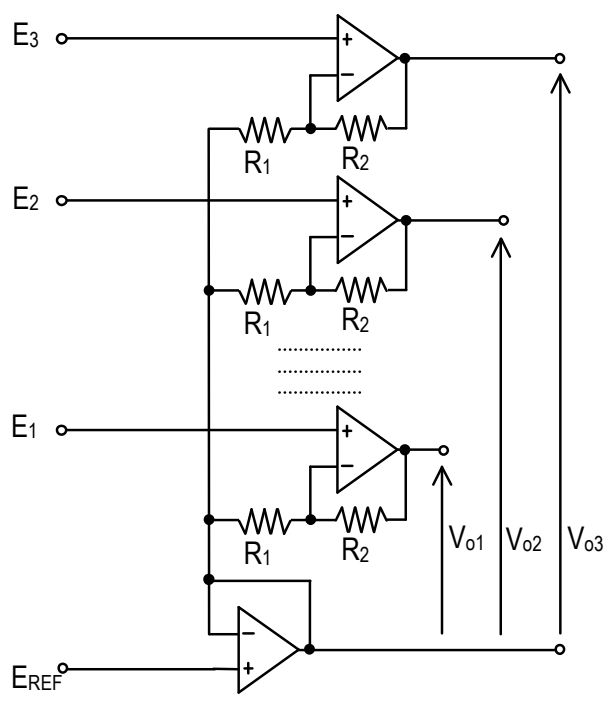

Figura 5.15. Topología de A.B. propuesta para configuración Monopolar.

Como puede observarse en la Fig.5.15, la impedancia de modo común $Z_{C}$ es elevada para todas las entradas. La misma está dada ${ }^{3}$ por la capacidad de entrada propia de los A.O. utilizados.

El comportamiento de este circuito puede analizarse a partir de su celda básica, la cual se muestra en la Fig.5.16.

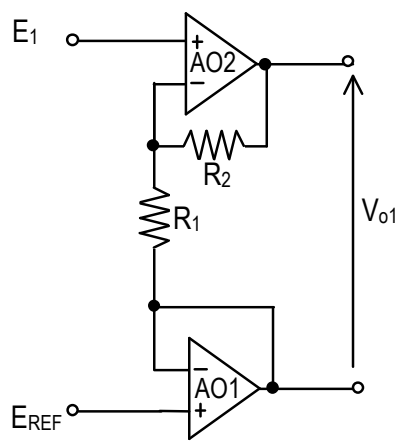

Figura 5.16. Celda Básica del Amplificador Monopolar propuesto.

${ }^{3}$ En realidad el A.O. del electrodo de referencia trabaja con una realimentación de tensión más fuerte que los restantes, pero este efecto no es significativo pues la impedancia de entrada está dominada por la capacidad de entrada de los A.O. 


\section{Funcionamiento.}

Este circuito no contiene ningún elemento a masa y por lo tanto su CMRR es idealmente infinito (APÉNDICE 5).

La corriente sobre $R_{1}$ depende exclusivamente de la tensión de modo diferencial ${ }^{4}$ y está dada por:

$$
i_{\mathrm{R} 1}=\frac{V_{\mathrm{iD}}}{R_{1}}
$$

Esta corriente circula por $R_{1}$ y por $R_{2}$, resultando una tensión de salida diferencial $V_{\mathrm{oD}}$.

$$
V_{\mathrm{oD}}=\frac{V_{\mathrm{iD}}}{R_{1}}\left(R_{1}+R_{2}\right)
$$

Es decir que la ganancia de modo diferencial $G_{\mathrm{DD}}$ está dada por:

$$
G_{\mathrm{DD}}=1+R_{2} / R_{1}
$$

La única corriente en el circuito es $i_{\mathrm{R} 1}$, la cual depende exclusivamente de la tensión diferencial de entrada. En el circuito no habrá corrientes ni tensiones dependientes de la tensión de modo común y por lo tanto su CMRR es idealmente infinito.

Considerando que los amplificadores $\mathrm{AO} 1, \mathrm{AO} 2$ tienen, respectivamente, rechazos de modo común $\mathrm{C}_{\mathrm{AO} 1} \mathrm{y}_{\mathrm{AO} 2}$, y siguiendo un procedimiento similar al utilizado en el APÉNDICE 6 para el amplificador F-D de dos A.O, resulta:

$$
C M R R^{-1}=C_{\mathrm{AO} 2}^{-1}-C_{\mathrm{AO} 1}^{-1}
$$

Esto parece demasiado bueno para este circuito, dado que su aspecto es desagradablemente asimétrico. De hecho, las primeras experiencias mostraron que su rechazo de modo común, en ciertas condiciones es significativamente menor que el predicho por (5.38). Evidentemente, el elemento que produce esta degradación no está presente en el modelo de la Fig.5.16. El bajo CMRR se debe, como se mostrará a continuación, a la impedancia de entrada propia de los A.O. Incorporando este elemento al circuito simplificado de la Fig.5.16 resulta:

\footnotetext{
${ }^{4}$ Para tensiones de modo común, ambos bornes de $R_{1}$ se encuentran, a la tensión de modo común de entrada $V_{\text {iC }}$ (esto es a través de las tierras virtuales establecidas entre las entradas de los A.O) y por lo tanto no circulará sobre ella ninguna corriente relacionada con $V_{\mathrm{iC}}$.
} 


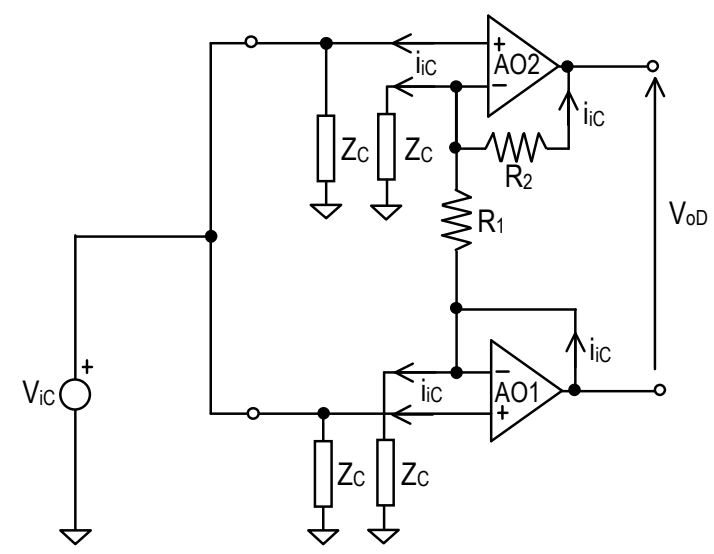

Figura 5.17. Circuito equivalente que incluye las impedancias $Z_{c}$ propias de los A.O.

Al aplicar una tensión de modo común, circularan corrientes $i_{\mathrm{iC}}=\left(V_{\mathrm{iC}} / Z_{\mathrm{C}}\right)$ sobre las impedancias de entrada de modo común $Z_{C}$ de cada uno de los A.O. Las corrientes correspondientes a las entradas no-inversoras son aportadas por el generador $V_{\mathrm{iC}}$ de entrada y no traen inconvenientes. Por otra parte, dado que la corriente sobre $R_{1}$ es nula, las $i_{\text {iC }}$ asociadas a las entradas inversoras circulan hacia la salida de los A.O. La correspondiente al buffer (AO1) encontrará la salida a través de un cortocircuito y no producirá diferencia de potencial alguna, pero la $i_{\mathrm{iC}}$ correspondiente a $\mathrm{AO} 2$ circulará sobre $R_{2}$ produciendo una tensión de salida de modo diferencial dada por:

$$
V_{\mathrm{oD}}=i_{\mathrm{iC}} R_{2}=V_{\mathrm{iC}} \frac{R_{2}}{Z_{C}}
$$

Es decir, que aún considerando A.O. ideales (CMRR y ganancia infinitas), la ganancia $G_{\mathrm{DC}}$ no es nula sino que está dada por:

$$
G_{\mathrm{DC}}=\frac{R_{2}}{Z_{\mathrm{C}}} \quad,
$$

y el rechazo de modo común resulta:

$$
C M R R=\frac{G_{\mathrm{DD}}}{G_{\mathrm{DC}}}=\frac{1+R_{2} / R_{1}}{R_{2}} Z_{\mathrm{C}}
$$

Si la ganancia diferencial es importante $\left(R_{2} / R_{1} \gg 1\right)$, esta expresión puede aproximarse por:

$$
C M R R=\frac{G_{\mathrm{DD}}}{G_{\mathrm{DC}}}=\frac{Z_{\mathrm{C}}}{R_{1}}
$$

Considerando ahora que $Z_{\mathrm{C}}$ está definida principalmente por la capacidad de entrada $C_{\mathrm{IN}}$ de los A.O., y teniendo en cuenta su CMRR, el rechazo de modo común total está dado aproximadamente por:

$$
\mathrm{CMRR}^{-1} \cong\left(C_{\mathrm{AO} 2}^{-1}-C_{\mathrm{AO} 1}^{-1}\right)+R_{1} \cdot C_{\mathrm{IN}} \cdot 2 \pi f
$$


Para frecuencias bajas dominará el primer término, mientras que para frecuencias altas el efecto de $C_{\mathrm{IN}}$ será preponderante.

\section{Resultados Experimentales.}

Para verificar el análisis previo, se construyó un amplificador para configuración monopolar según la topología de la Fig.5.15 y con una ganancia $G_{\mathrm{DD}}=100$. En su implementación se utilizaron amplificadores operacionales TLC220 (estos A.O. presentan una $C_{\mathrm{IN}}$ relativamente alta $\left.\approx 18 \mathrm{pF}\right)$ y se midió el CMRR para distintos valores de $R_{1}\left(R_{1}=220 \Omega, 2.2 \mathrm{k} \Omega, 22 \mathrm{k} \Omega\right)$.

De acuerdo a (5.43), para altas frecuencias, el CMRR de este A.B. resulta:

$$
\mathrm{CMRR}^{-1}=R_{1} \cdot 18 \mathrm{pF} \cdot 2 \pi f \quad .
$$

En la Fig.5.18 se muestran los resultados experimentales y el CMRR dado por (5.44) para distintos valores de $R_{1}$. Se observa claramente que, para altas frecuencias, donde este efecto es dominante, el CMRR sigue muy aproximadamente la expresión (5.44).

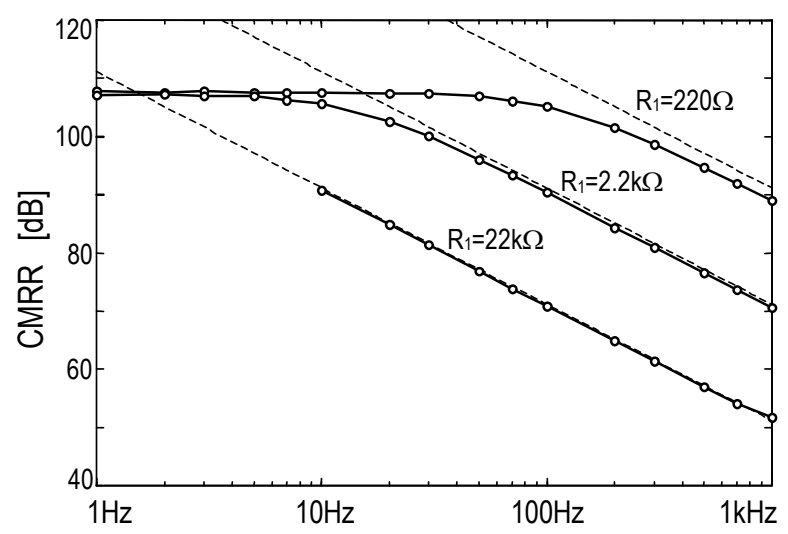

Figura 5.18. CMRR del circuito de Fig. 5.16 para distintos valores de $R_{1}$. Los resultados experimentales están indicados con símbolos y el CMRR teórico según la expresión (5.44) en línea punteada.

Para que el efecto de $R_{1}$ a $50 \mathrm{~Hz}$. no sea apreciable, el CMRR debido a este mecanismo debe ser de unos $110 \mathrm{~dB}$ o superior ${ }^{5}$. Dado un CMRR requerido a $50 \mathrm{~Hz}$, el valor máximo de $R_{1}$ admisible queda dado por:

$$
R_{1}=\frac{1}{\left.\mathrm{CMRR}\right|_{50 \mathrm{~Hz}} 2 \pi 50 \mathrm{~Hz} \cdot C_{\mathrm{IN}}} .
$$

Por ejemplo, continuando con el mismo A.O. $\left(C_{\mathrm{IN}} \approx 18 \mathrm{pF}\right)$, si se apunta a un CMRR $>110 \mathrm{~dB}$ es suficiente con utilizar un resistor $R_{1}$ menor de $560 \Omega$. Esto no es

\footnotetext{
${ }^{5}$ El rechazo de un F-D de dos A.O. en general es de aproximadamente este valor (110 dB)
} 
difícil de cumplir pero es un punto a tener en cuenta, pues muchas veces, para asegurar un bajo consumo, es usual utilizar resistores de valores algo más altos, de hasta algunos ${ }^{6} \mathrm{k} \Omega$. Tampoco es aconsejable utilizar valores de $R_{1}$ extremadamente bajos, pues en presencia tensiones de modo diferencial de entrada elevadas (ej. Tensión de offset de los electrodos) el consumo se incrementaría significativamente.

\section{Amplificadores para sistemas de 2 Electrodos.}

Las condiciones de diseño para amplificadores de dos electrodos son particulares. Como no existe un electrodo de masa que vincule fuertemente los potenciales del paciente con los del A.B, la tensión de modo común $V_{\text {ic }}$ queda definida sobre la impedancia de entrada de modo común $Z_{\mathrm{C}}$. En la Fig.5.19 se muestra un esquema que describe esta situación.

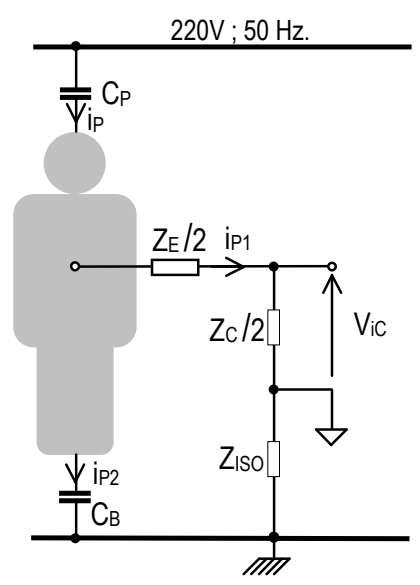

Figura 5.19. Esquema simplificado de la situación de EMI en sistemas de 2 Electrodos, válido para tensiones de Modo Común.

La corriente de desplazamiento $i_{\mathrm{P}}$ se distribuirá, de acuerdo al valor de las impedancias consignadas en la Fig.5.19, en una componente $i_{\mathrm{P} 1}$ que circulará sobre la impedancia de entrada de modo común $Z_{\mathrm{C}}$ y otra $i_{\mathrm{P} 2}$ que derivará a tierra a través de la capacidad distribuida $C_{\mathrm{B}}$. El peor caso, desde el punto de vista del rango de entrada del A.B., se produce cuando toda la $i_{\mathrm{P}}$ circula sobre $Z_{\mathrm{C}}{ }^{7}$. En este caso, la tensión de modo común $V_{\text {iC }}$ está dada por:

$$
V_{\mathrm{iC}}=i_{\mathrm{P}} \cdot Z_{\mathrm{C}} / 2
$$

\footnotetext{
${ }^{6}$ Tampoco mucho mayores. Como referencia, un resistor de $6 \mathrm{k} \Omega$ aporta un nivel de ruido comparable a un buen A.O. $(10 \mathrm{nV} / \sqrt{ } \mathrm{Hz})$

${ }^{7}$ Esta es una situación verdaderamente desfavorable, pues en general los sistemas de dos electrodos se utilizan en pequeños amplificadores alimentados a batería que presentan muy altas impedancias de aislamiento $Z_{\text {ISO }}$ y solo una pequeña fracción de $i_{\mathrm{P}}$ circulará sobre $Z_{\mathrm{C}}$. Por ejemplo, en aplicaciones telemétricas, donde es posible conseguir muy altas $Z_{\text {ISO }}$, las condiciones de medida se relajan al extremo de ser factible utilizar un simple amplificador no inversor Single-Ended (Dobrev, 1998)
} 
Dada una cierta $i_{\mathrm{P}}$, para conseguir que la tensión $V_{\mathrm{iC}}$ no supere el rango de entrada del amplificador, es necesario limitar el máximo valor de $Z_{\mathrm{C}}$. Por otra parte, como se mostró en el CAPítUlO 4, una baja $Z_{\mathrm{C}}$ también puede resultar conveniente desde el punto de vista del rechazo a la tensión de línea. Por todo esto, una baja $Z_{\mathrm{C}}$ resulta una alternativa a tener en cuenta para amplificadores de 2 electrodos.

La corriente $i_{\mathrm{P}}$ usualmente no es mayor de $1 \mu \mathrm{A}$ (SERRANO, 2003). Adoptando este valor y considerando una tensión de alimentación de $\pm 3 \mathrm{~V}$, la máxima $\mathrm{Z}_{\mathrm{C}}$ admisible resulta de unos $3 \mathrm{M} \Omega$.

\subsection{Topologías para A.B. con baja impedancia de entrada de modo común $Z_{c}$.}

Si se adopta un modelo pasivo clásico para las impedancias de entrada del A.B. (Fig.5.20.a), no resulta posible conseguir bajas $Z_{C}$ sin degradar la impedancia de entrada de modo diferencial $Z_{\mathrm{D}}$. Para sortear esto, es necesario implementar soluciones activas como las presentadas en el CAPÍTULO 3 que por comodidad se repiten aquí en las Fig.5.20.(b) y (c). Estos esquemas permiten conseguir muy bajas $Z_{\mathrm{C}}$ manteniendo elevadas $Z_{\mathrm{D}}$.

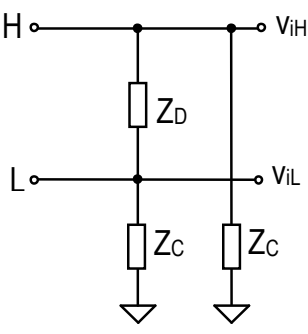

(a)

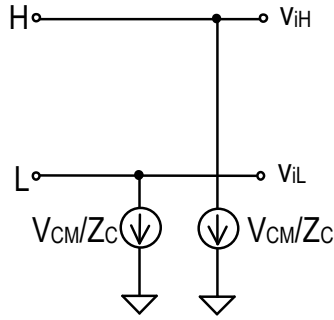

(b)

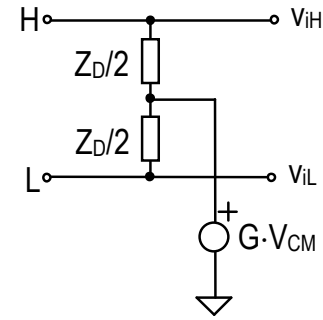

(c)

Figura 5.20.(a) Circuito Clásico para representar las impedancias de entrada de un amplificador diferencial. (b) y (c) Circuitos alternativos para conseguir bajas $Z_{C}$ manteniendo elevadas $Z_{D}$.

La topología de la Fig.5.20.(b) fue propuesta ${ }^{8}$ y desarrollada recientemente por Dobrev (Dobrev, 2002a) (Dobrev, 2002b) (Dobrev, 2004). Esta topología presenta la ventaja de admitir importantes corrientes de entrada de modo común $i_{\mathrm{P}}$, de hasta decenas de $\mu \mathrm{A}$, pero su implementación es algo compleja y requiere componentes apareados.

En esta tesis se propone un circuito de acuerdo a la topología de la Fig.5.20.(c). El circuito propuesto permite conseguir muy bajas $Z_{\mathrm{C}}$ (de cientos de $\Omega$ ) con una implementación muy simple. Si bien admite corrientes $i_{\mathrm{P}}$ sensiblemente menores que el circuito propuesto en (Dobrev, 2002a), su capacidad resulta suficiente para operar en condiciones reales. La impedancia de entrada de modo común para el circuito de la Fig.5.20.(c), está dada por:

\footnotetext{
${ }^{8}$ En realidad la idea de este circuito es antigua y se utiliza comúnmente en telefonía para la interfaz de abonado (SLIC: Suscriber Line Interface Circuit) a fin de conseguir bajas $Z_{\mathrm{C}}$ manteniendo una alta $Z_{\mathrm{D}}$. Dobrev propuso su uso en aplicaciones biomédicas.
} 


$$
Z_{\mathrm{C}}=\frac{Z_{\mathrm{D}} / 2}{1-G}
$$

Se advierte aquí una fuerte vinculación entre $Z_{\mathrm{C}}$ y $Z_{\mathrm{D}}$, pero operando sobre la ganancia $G$ se consigue una gran libertad para definir esta relación. Por ejemplo, utilizando una ganancia negativa y muy elevada, es posible conseguir muy bajas $Z_{\mathrm{C}}$. Como la realimentación no afecta a las tensiones de modo diferencial, la impedancia de entrada para señales de modo diferencial se mantiene igual a $Z_{\mathrm{D}}$. Un circuito práctico para implementar esta estrategia se muestra en la Fig.5.21.

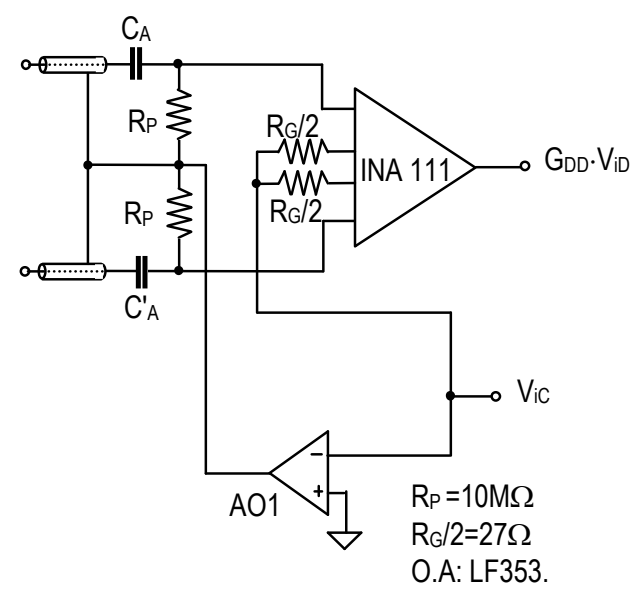

Figura 5.21. Circuito práctico para implementar la topología de la Fig. 5.21.c.

De acuerdo a (5.47), considerando que el A.O. utilizado (LF353) presenta una ganancia a lazo abierto a $50 \mathrm{~Hz}$ de unos $100 \mathrm{~dB}\left(\mathrm{G}=10^{5}\right)$, utilizando $R_{\mathrm{P}}=10 \mathrm{M} \Omega$, resulta una $Z_{\mathrm{C}}$ tan baja como $100 \Omega$, mientras que la impedancia de entrada para señales de tipo diferencial se mantiene en $20 \mathrm{M} \Omega$. Este circuito fue utilizado en los ensayos con amplificadores para dos electrodos del CAPÍTULO 3.

\subsubsection{Rango de Entrada.}

La realimentación de modo común tiende a mantener una $V_{\text {ic }}$ nula. Dado que la excursión salida de $\mathrm{AO} 1$ está limitada a $\pm V_{\mathrm{CC}}$ la máxima corriente $i_{\mathrm{P}}$ admisible (ver Fig.5.19) se produce cuando:

$$
\hat{i_{\mathrm{P}}} / 2 \cdot R_{\mathrm{P}}=V_{\mathrm{CC}},
$$

es decir que la máxima $i_{\mathrm{P}}$ admisible está dada por:

$$
\hat{i}_{\mathrm{P} . \mathrm{MAX}}=\frac{2 V_{\mathrm{CC}}}{R_{\mathrm{P}}}
$$


Por ejemplo, considerando $V_{\mathrm{CC}}= \pm 3 \mathrm{~V}$ y una impedancia de entrada de modo diferencial $Z_{\mathrm{D}}=2 R_{\mathrm{P}}=20 \mathrm{M} \Omega$, resulta una $i_{\mathrm{P}}$ máxima admisible de $1.2 \mu \mathrm{App} \quad\left(\approx 850 \mathrm{nA}_{\mathrm{RMS}}\right)$, suficiente para asegurar el funcionamiento aún en condiciones de EMI agresivas. Este valor de $i_{\mathrm{P}}$ equivale a una capacidad $C_{\mathrm{P}}$ tan alta como $12 \mathrm{pF}$, lo cual significa que el paciente se encuentra tocando un cable de línea (220V) aislado (SERRANO, 2003).

\section{Conclusiones.}

Sistemas de tres electrodos (con electrodo de masa) - Configuración Bipolar.

Para este tipo de sistemas, la topología más apropiada es el amplificador F-D de dos A.O., que permite conseguir elevados CMRR sin requerir elementos apareados. Este circuito presenta además un excelente rango de entrada siendo esta última característica particularmente importante en A.B. alimentados a baterías.

El amplificador de instrumentación clásico de 3 A.O., así también como el de 2 A.O., presentan CMRR comparables al F-D de 2 A.O. siempre que se les asigne una ganancia importante. Esto no siempre es posible debido a los potenciales de continua que se producen en la interfase electrodo-piel cuyas diferencias se presentan al A.B. como tensiones de modo diferencial.

En cuanto a la tensión equivalente de ruido, la misma es similar para las tres topologías mencionadas y está dada por el ruido equivalente correspondiente a dos A.O.

Sistemas de tres electrodos (con electrodo de masa) - Configuración Monopolar.

En un A.B. para configuración monopolar de electrodos es importante mantener el balance entre las impedancias de entrada de modo común $Z_{\mathrm{C}}$ de cada una de sus entradas. Una forma simple de conseguirlo es utilizando buffers en las entradas del A.B, pero esto incrementa el nivel de ruido del amplificador. Para evitar este inconveniente, se desarrolló una topología que proporciona ganancia e impedancias de entrada balanceadas. La misma permite alcanzar elevados CMRR sin requerir elementos apareados.

Sistemas de dos electrodos (sin electrodo de masa).

En este tipo de mediciones resulta conveniente utilizar A.B. con bajas impedancias de entrada de modo común $Z_{\mathrm{C}}$. Se propuso un circuito simple, basado en la realimentación de la tensión de modo común, que permite conseguir una baja $Z_{\mathrm{C}}$ manteniendo altas impedancias de entrada $Z_{\mathrm{D}}$ para tensiones de modo diferencial. 


\section{Capítulo 6: Acoplamiento en Alterna de Amplificadores de Biopotenciales.}

\section{Resumen.}

Los principales inconvenientes en la amplificación de biopotenciales se deben a las tensiones de continua (DC) que se originan en la interfase electrodo-piel. Estas componentes de DC pueden llegar a ser de centenas de milivolts, es decir varios órdenes de magnitud mayores que las señales bioeléctricas y aparecen superpuestas a la señal de interés.

La solución clásica a este inconveniente consiste en utilizar una primer etapa acoplada en DC con una ganancia apenas moderada a fin de evitar su saturación. Posteriormente, ya con la señal referida a masa (single-ended), se realiza el acoplamiento en alterna (AC) y se incorporan las siguientes etapas necesarias para conseguir la ganancia deseada.

Como se vio en los capítulos previos, para conseguir altos CMRR y bajos niveles de ruido es importante asignar una ganancia elevada a la primer etapa. En este capítulo, se proponen soluciones al acoplamiento en $\mathrm{AC}$ que permitan asignar una ganancia importante a la etapa de entrada sin degradar su CMRR ni su impedancia entrada para señales de modo común. 


\section{Acoplamiento en Alterna.}

El bloqueo de las tensiones de DC es tal vez la característica más representativa del diseño de amplificadores para biopotenciales. Para poder implementar altas ganancias en la primer etapa es necesario "bloquear" estas componentes de baja frecuencia. También es importante que las soluciones propuestas mantengan una muy alta impedancia de entrada de modo común $Z_{C}$, a fin de no degradar el rechazo a la interferencia de la red.

Algunos de los factores de mérito de un acoplamiento en AC para amplificadores de biopotenciales son:

- Rango de tensiones de DC admisibles a la entrada

- Rechazo de Modo Común (CMRR)

- Offset de DC a la salida.

- Máxima ganancia admisible en la primer etapa.

- Impedancia de entrada de Modo Común $\mathrm{Z}_{\mathrm{C}}$.

- Consumo.

\section{Preservación del CMRR}

Uno de los principales objetivos de diseño, es conseguir el acoplamiento en AC preservando el CMRR del amplificador. Pensando en soluciones que puedan aplicarse directamente a la entrada, los circuitos deberán ser de tipo full-diferencial (F-D), dado que tanto la señal bioeléctrica (entrada a la red) como la entrada del amplificador (salida de la red) son de tipo diferencial.

En la implementación de circuitos en general, es deseable evitar la necesidad de ajustes y el uso de componentes con muy bajas tolerancias. Un par de posibilidades para conseguir elevados CMRR independientemente de los desbalances en los componentes del circuito se muestran en las Fig.6.1.(a) y Fig.6.1.(b).

La primera de ellas (Fig.6.1.(a)) consiste en conseguir que el circuito F-D sea flotante, es decir sin conexión alguna a masa. De este modo, si se aplica una tensión de entrada de modo común, no se establece ningún camino cerrado y por lo tanto no circularan corrientes sobre el circuito. Tampoco aparecerán diferencias de potencial sobre él; la tensión diferencial de salida $V_{\mathrm{oD}}$ será nula y el CMRR idealmente infinito. Esta solución es apropiada para redes pasivas, pero puede resultar difícil de aplicar en circuitos activos, debido a que en este caso se requieren caminos a masa para las corrientes de polarización de entrada de los amplificadores operacionales.

La segunda solución, ilustrada en la Fig.6.1.(b), admite conexiones del circuito a masa pero exclusivamente a través de generadores de tensión de valor idéntico a la tensión de modo común de entrada. Al aplicar una tensión de entrada de modo común $V_{\mathrm{iC}}$, todas las mallas cerradas que se puedan establecer presentaran una tensión neta de fuentes nula, por lo tanto todas las corrientes de estas mallas serán idénticamente nulas así como la tensión diferencial de salida $V_{\mathrm{oD}}$ : el CMRR resulta idealmente infinito. En el APÉNDICE 5 se incluye un análisis detallado de estas posibilidades. 
En resumen, para conseguir acoplamiento en AC sin degradar el CMRR, existen al menos dos alternativas:

a) Utilizar una red sin conexión alguna a masa (Flotante)

b) Utilizar una red cuyas conexiones a masa se realicen mediante generadores de tensión iguales a la tensión de modo común de entrada.

En las secciones siguientes se proponen algunas estrategias que hacen uso de estas observaciones.

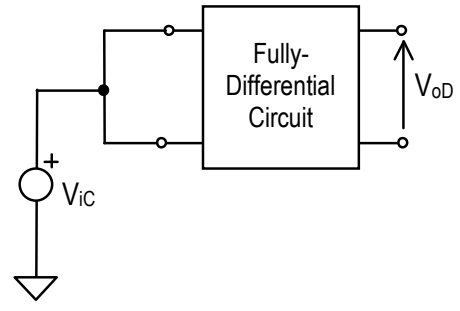

(a)

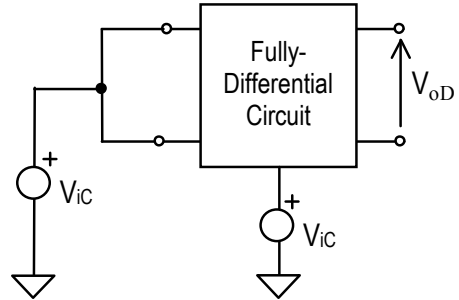

(b)

Figura 6.1. Dos formas posibles de conseguir un CMRR infinito en Redes F-D. (a) Una red flotante. (b) Una red conectada a masa mediante generador de tensión de valor idéntico a la tensión de mdo común de entrada Vic.

\section{Acoplamiento AC mediante redes pasivas}

La forma más simple y económica de eliminar las componentes de DC es acoplando la señal mediante capacitores. Este tipo de técnica, que solo requiere una red de entrada apropiada, compuesta por componentes pasivos, se denomina "acoplamiento en alterna pasivo".

Como se indicó anteriormente, es deseable que el acoplamiento en $\mathrm{AC}$ se realice directamente a la entrada del amplificador, a fin de poder asignar una ganancia importante a su primer etapa. Esto redundará en altos CMRR sin requerir componentes apareados y en bajos niveles de ruido. Como contraparte, al no incluir ninguna etapa separadora previa, es necesario trabajar con altos desbalances entre las impedancias de generador, las cuales están determinadas por las poco predecibles impedancias electrodo-piel. Esto último exige muy altas impedancias de entrada de modo común $Z_{C}$ para evitar que debido al "efecto divisor de potencial", resulte un pobre rechazo de la tensión de red. La tensión diferencial de entrada equivalente $V_{\mathrm{iD}}$, debida este mecanismo (APÉNDICE 2), está dada por:

$$
V_{\mathrm{iD}} \cong V_{\mathrm{iC}} \cdot \frac{Z_{E}}{Z_{\mathrm{C}}}\left\{\frac{\Delta Z_{\mathrm{E}}}{Z_{\mathrm{E}}}-\frac{\Delta Z_{\mathrm{C}}}{Z_{\mathrm{C}}}\right\} \quad \text {. }
$$


Como en general el desbalance en las impedancias de electrodo es mucho mayor que el correspondiente a las impedancias de modo común, el primer efecto es dominante y (6.1) puede aproximarse por:

$$
V_{\mathrm{iD}} \cong V_{\mathrm{iC}} \cdot \frac{\Delta Z_{E}}{Z_{\mathrm{C}}}
$$

Es posible definir un Rechazo de Modo Común equivalente $C M R R_{\mathrm{DP}}$ debido al efecto divisor de potencial como:

$$
C M R R_{\mathrm{DP}}^{-1}=\frac{\Delta Z_{\mathrm{E}}}{Z_{\mathrm{C}}}
$$

El principal inconveniente de las soluciones pasivas es que los capacitores de acoplamiento bloquean tanto las componentes de DC de la señal como las correspondientes a las corrientes de polarización propias del amplificador. Esto obliga a incluir resistores conectados a masa para proveer caminos de DC alternativos, degradando $\mathrm{Z}_{\mathrm{C}} \mathrm{y}$ conduciendo a bajos $C M R R_{\mathrm{DP}}$.

A continuación se analizan algunos circuitos clásicos y se propone una nueva red que permite implementar el acoplamiento en AC sin degradar la impedancia de entrada del amplificador para tensiones de modo común.

\subsection{Resistores de polarización simples conectados a masa.}

La red de acoplamiento debe intercalarse entre la señal bioeléctrica que es de tipo diferencial y la entrada del A.B. que también es diferencial; es decir que la red en cuestion debe ser Fully-Differential (F-D).

El circuito más simple para conseguir acoplamiento en AC, se obtiene por "espejado" de un filtro pasa-altos de primer orden de tipo single-ended (S-E), resultando el circuito balanceado de la Fig. 6.2.

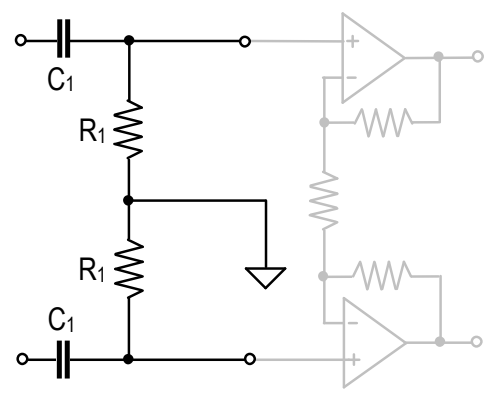

Figura 6.2. Red de acoplamiento en AC que incorpora resistores a masa para proveer caminos a las corrientes de poalrización.

Las impedancias de entrada para señales de modo común y de modo diferencial, están dadas por:

$$
Z_{C}=R_{1} \quad ; \quad Z_{D}=2 R_{1}
$$


Este circuito no resulta apropiado para ser utilizado en un A.B., porque implementado utilizando componentes estándar, su $Z_{\mathrm{C}}$ está limitada a algunas decenas de $\mathrm{M} \Omega$.

\subsection{Red de polarización "T"}

Una mejora al circuito previo consiste en no conectar los resistores $R_{1}$ directamente a masa sino a través de un resistor $R_{2}$ adicional según se muestra en la Fig.6.3.(a).

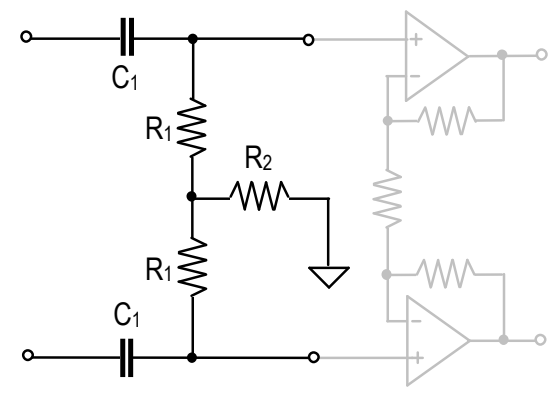

Figura 6.3. Mejora sobre el circuito de la Fig. 6.2 que consiste en utilizar una red T para elevar la impedancia de entrada de modo común Zc.

El resistor $R_{2}$ incrementa la impedancia de modo común y las impedancias de entrada de esta red resultan:

$$
Z_{C}=R_{1}+2 R_{2} \quad ; \quad Z_{D}=2 R_{1}
$$

El valor de $Z_{\mathrm{C}}$ que permite alcanzar este circuito tampoco resulta suficiente para utilizarlo directamente a la entrada del amplificador, es decir enfrentando los desbalances usuales entre las impedancias de electrodo. Esta red sí puede resultar una alternativa válida para el acoplamiento entre etapas diferenciales internas de un A.B., donde las impedancias de generador están controladas (SPINELLI, 2001).

\subsection{Simulación de elevadas $Z_{c}$.}

Una forma de mejorar la $Z_{\mathrm{C}}$ de la red del punto previo, es simulando un resistor $R_{2}$ de valor muy elevado mediante un circuito activo que incluye una red "T" en la realimentación (Fig.6.4) (RAMOS, 1999). Si bien este esquema incluye elementos activos (un A.O.), conceptualmente su funcionamiento corresponde a un acoplamiento pasivo y por eso se lo analiza en esta sección.

El circuito que simula a $R_{2}$ muestra una resistencia equivalente $R_{\mathrm{EQ}}$ (ver APÉNDICE 4) que esta dada aproximadamente por:

$$
R_{E Q} \cong R_{\mathrm{A}} R_{\mathrm{C}} / R_{\mathrm{B}}
$$




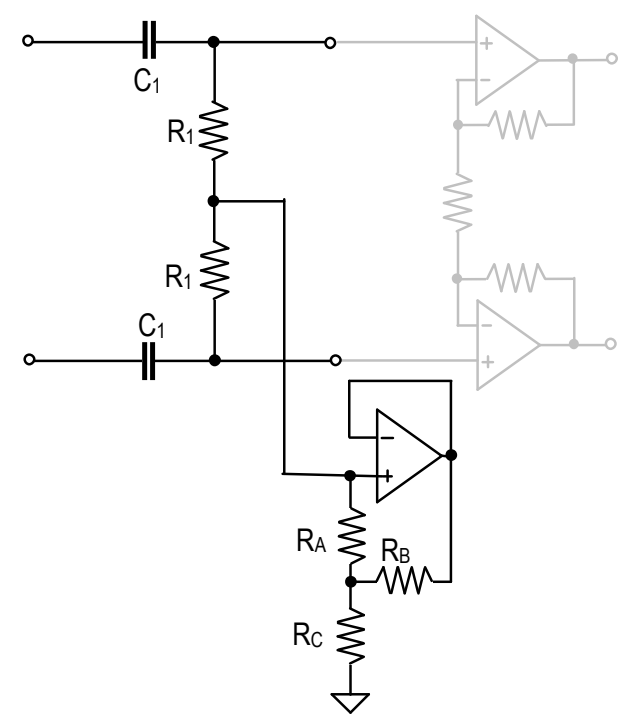

Figura 6.4. Circuito que utiliza bootstraping para simular un resistor $\mathrm{R}_{2}$ (ver Fig. 6.3) de valor elevado.

Con esta configuración pueden obtenerse, impedancias $Z_{\mathrm{C}}$ de hasta algún G $\Omega$ (CASAS, 1996). Por ejemplo, considerando $\mathrm{Z}_{\mathrm{C}}=1 \mathrm{G} \Omega$, el $C M R R_{\mathrm{DP}}$ dado por (6.2), para $Z_{\mathrm{E}}=100 \mathrm{k} \Omega$ y desbalances del $50 \%$, resulta de:

$$
C M R R_{\mathrm{DP}}=\frac{1 \mathrm{G} \Omega / 100 \mathrm{k} \Omega}{0.5} \cong 86 \mathrm{~dB}
$$

Un valor que puede considerarse aceptable. Para incrementar $C M R R_{\mathrm{DP}}$, manteniendo esta topología, es necesario elevar aún más $R_{\mathrm{EQ}}$, lo cual desnudaría algunos problemas de este circuito, como el desplazamiento del nivel de DC debido a las corrientes de polarización y la amplificación del offset del A.O. (esto se analiza en el APÉNDICE 4).

\subsection{Una nueva Red de Acoplamiento Flotante.}

Los inconvenientes de los circuitos analizados previamente nacen en las impedancias que los vinculan a masa. Como se mostró en la sección 3 de este capítulo, si se consigue implementar el acoplamiento en AC mediante una red F-D perfectamente flotante, el $C M R R_{\mathrm{DP}}$ será idealmente infinito. En este caso, la red no degradará en absoluto las características propias del amplificador que precede.

En esta tesis se propone una nueva red de acoplamiento pasiva y totalmente flotante (SPINELLI, 2003.a). La misma se presenta en la Fig.6.5. (SPINELLI, 2003.a). 


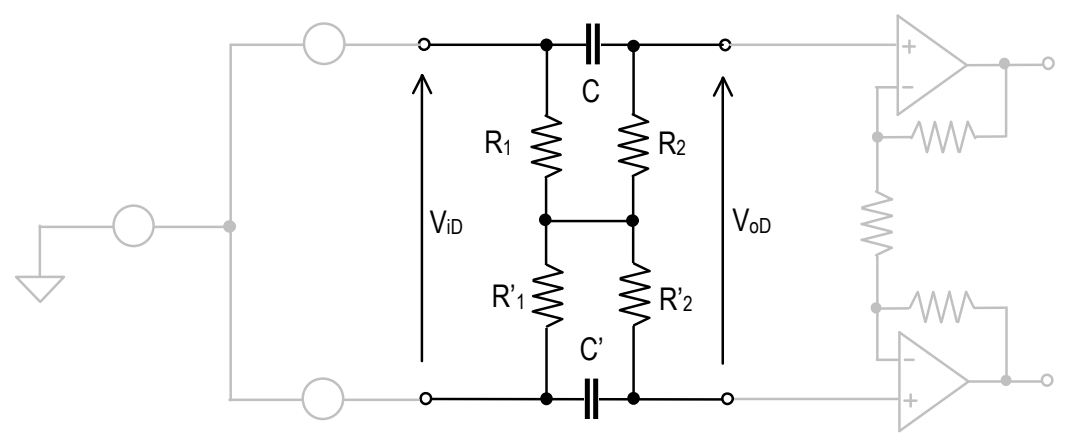

Figura 6.5. Circuito propuesto para acoplamiento en alterna pasivo.

Esta red bloquea la componente de DC de modo diferencial y provee un camino de DC a las corrientes de polarización del A.O. Estas últimas encuentran su camino a masa a través del generador de señal de entrada.

El circuito propuesto es de tipo F-D y no contiene ningún elemento conectado a masa; por lo tanto su $C M R R_{\mathrm{DP}}$ será idealmente infinito e independientemente de los desbalances que pudieran existir entre los elementos que la componen.

Si bien esta red no presenta ninguna vinculación con masa, igualmente brinda un camino a las corrientes de polarización de los A.O. que, circulando por $R_{1}, R_{2}, R_{1}{ }_{1} \mathrm{y}$ $R^{\prime}{ }_{2}$, encuentran su camino a masa través de las impedancias de los electrodos. En esta solución, al igual que en la mayoría de los amplificadores para biopotenciales, las corrientes de polarización circulan a través del paciente ${ }^{1}$. Para observar esto más claramente, en la Fig.6.6 se muestra un esquema que incluye al paciente y al electrodo de masa $Z_{\mathrm{E} 3}$.

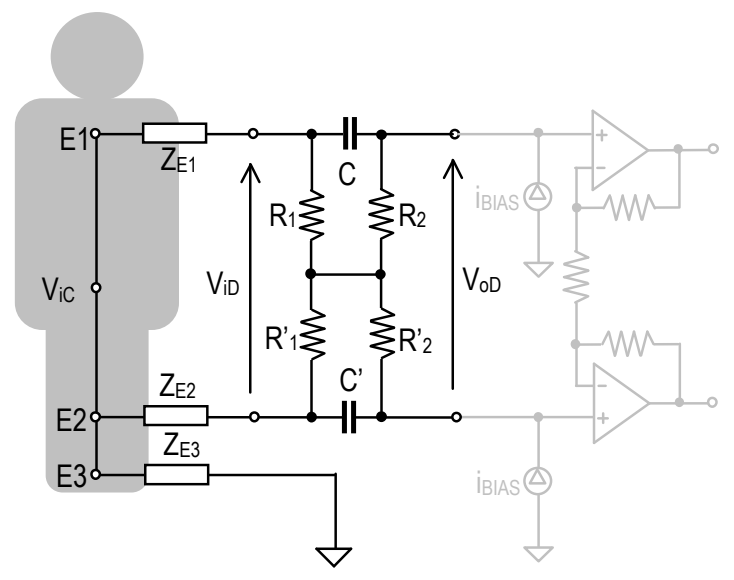

Figura 6.6. Red de Acoplamiento propuesta aplicada en un A.B. Se observa que las corrientes de polarización circulan sobre el paciente y encuentran su camino a masa a través de la impedancia del tercer electrodo ZE3. Esto mismo ocurre cuando se utiliza una primer etapa acoplada en DC (la topología clásica más habitual)

\footnotetext{
${ }^{1}$ La norma AAMI limita las corrientes de DC sobre el paciente a $10 \mu \mathrm{A}$, algo muy fácil de cumplir con los A.O. actuales.
} 


\subsubsection{Componentes perfectamente apareados.}

Si la red de la Fig.6.5 se considera perfectamente simétrica, su análisis puede realizarse aplicando el teorema de la bisección. El mismo conduce al circuito equivalente, válido para tensiones de modo diferencial, de la Fig.6.7 (b).

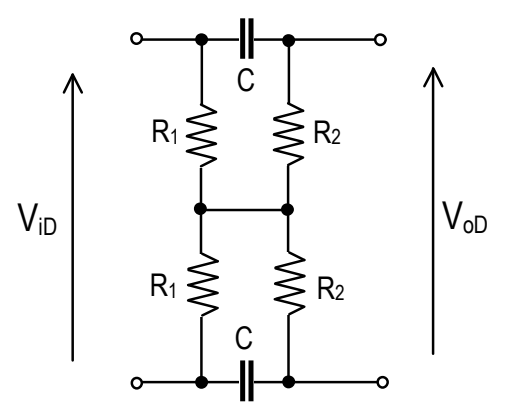

(a)

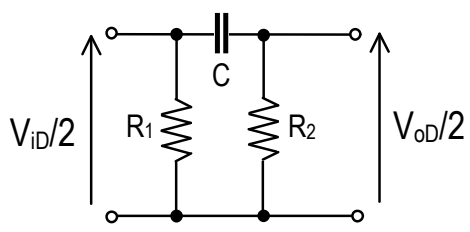

(b)

Figura 6.7. (a) Red de Acoplamiento propuesta. (b) Circuito equivalente válido para tensiones de modo diferencial suponiendo un balance perfecto entre los componentes.

En este caso, la función transferencia $G_{\mathrm{DD}}(s)=V_{\mathrm{oD}} / V_{\mathrm{iD}}$ se reduce a:

$$
G_{\mathrm{DD}}(s)=\frac{s}{s+1 / \tau_{2}} \quad ; \quad \tau_{2}=R_{2} C=R_{2}^{\prime} C
$$

y el circuito se comporta como un filtro pasa-altos de primer orden.

\subsubsection{Efecto de las tolerancias en los componentes.}

Los desapareamientos entre los componentes no afectan el CMRR pero, como se mostrará a continuación, sí influyen en la ganancia de modo diferencial $G_{\mathrm{DD}}$.

Si se considera $R_{1} \neq R^{\prime}{ }_{1}, R_{2} \neq R^{\prime}$ y $C \neq C$, no hay muchas posibilidades de utilizar sutilezas para analizar este circuito: su análisis es irremediablemente tedioso. Si se aplica una transformación triángulo-estrella, el circuito propuesto adopta la forma indicada en la Fig.6.8.(b).

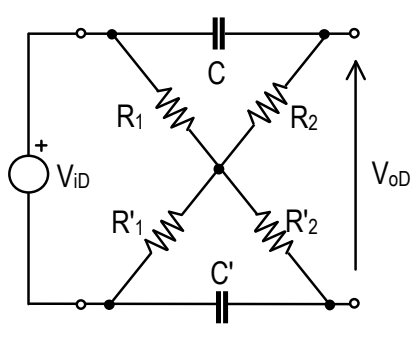

(a)

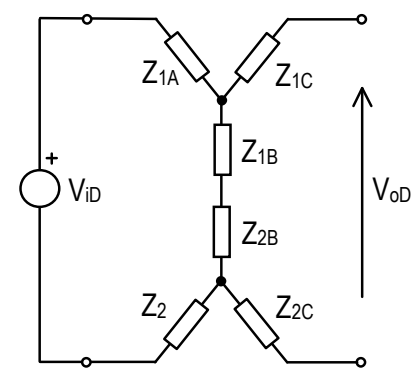

(b)

Figura 6.8. (a) Esquema de la red propuesta . (b) Circuito resultante de aplicar transformaciones triángulo- estrella. 
Siendo:

$$
\begin{aligned}
& Z_{1 \mathrm{~A}}=\frac{R_{1}}{1+s \cdot C_{1}\left(R_{1}+R_{2}\right)} ; Z_{1 \mathrm{~B}}=\frac{s \cdot R_{1} R_{2} C_{1}}{1+s \cdot C_{1}\left(R_{1}+R_{2}\right)} ; Z_{1 \mathrm{C}}=\frac{R_{2}}{1+s \cdot C_{1}\left(R_{1}+R_{2}\right)} \\
& Z_{2 \mathrm{~A}}=\frac{R_{1}^{\prime}}{1+s \cdot C_{1}^{\prime}\left(R_{1}^{\prime}+R_{2}^{\prime}\right)} ; Z_{2 \mathrm{~B}}=\frac{s \cdot R_{1}^{\prime} R_{2}^{\prime} C_{1}^{\prime}}{1+s \cdot C_{1}^{\prime}\left(R_{1}^{\prime}+R_{2}^{\prime}\right)} ; Z_{2 \mathrm{C}}=\frac{R_{2}^{\prime}}{1+s \cdot C_{1}^{\prime}\left(R_{1}^{\prime}+R_{2}^{\prime}\right)}
\end{aligned}
$$

Resolviendo el circuito de la Fig.6.8.(b), la función de transferencia $G_{\mathrm{DD}}(\mathrm{s})$ resulta:

$$
G_{\mathrm{DD}}(s)=\frac{Z_{1 \mathrm{~B}}+Z_{2 \mathrm{~B}}}{Z_{1 \mathrm{~A}}+Z_{1 \mathrm{~B}}+Z_{2 \mathrm{~A}}+Z_{2 \mathrm{~B}}}
$$

Reemplazando en (6.10) la expresiones indicadas en (6.9):

$$
G_{\mathrm{DD}}(s)=\frac{s \cdot R_{1} R_{2} C_{1}\left(1+s \cdot C_{1}^{\prime}\left(R_{1}^{\prime}+R_{2}^{\prime}\right)\right)+s \cdot R_{1}^{\prime} R_{2}^{\prime} C_{1}^{\prime}\left(1+s \cdot C_{1}\left(R_{1}+R_{2}\right)\right)}{\left(R_{1}+s \cdot R_{1} R_{2} C_{1}\right) \cdot\left(1+s \cdot C_{1}^{\prime}\left(R_{1}^{\prime}+R_{2}^{\prime}\right)\right)+\left(R_{1}^{\prime}+s \cdot R_{1}^{\prime} R_{2}^{\prime} C_{1}^{\prime}\right) \cdot\left(1+s \cdot C_{1}\left(R_{1}+R_{2}\right)\right)},
$$

Desarrollando el numerador y el denominador de (6.11), se obtiene:

$$
\begin{aligned}
& G_{\mathrm{DD}}(s)= \\
& \frac{s \cdot\left[\left(R_{1} R_{2} C_{1}+R_{1}^{\prime} R_{2}^{\prime} C_{1}^{\prime}\right)+s \cdot\left(C_{1} C_{1}^{\prime} R_{1} R_{2}\left(R_{1}^{\prime}+R_{2}^{\prime}\right)+R_{1}^{\prime} R_{2}^{\prime}\left(R_{1}+R_{2}\right) C_{1} C_{1}^{\prime}\right)\right]}{s^{2}\left[C_{1} C_{1}^{\prime} R_{1} R_{2}\left(R_{1}^{\prime}+R_{2}^{\prime}\right)+R_{1}^{\prime} R_{2}^{\prime}\left(R_{1}+R_{2}\right) C_{1} C_{1}^{\prime}\right]+s\left[R_{1} R_{2} C_{1}+R_{1} C_{1}^{\prime}\left(R_{1}^{\prime}+R_{2}^{\prime}\right)+R_{1}^{\prime} C_{1}\left(R_{1}+R_{2}\right)+R_{1}^{\prime} R_{2}^{\prime} C_{1}^{\prime}\right]+\left[R_{1}+R_{1}^{\prime}\right]}
\end{aligned}
$$

Llamando $\tau_{1}=R_{1} C_{1} ; \tau_{1}^{\prime}=R_{1}^{\prime} C_{1}^{\prime} ; \tau_{2}=R_{2} C_{1} ; \tau_{2}^{\prime}=R_{2}^{\prime} C_{1}^{\prime},(6.12)$ puede rescribirse como:

$$
\begin{aligned}
& G_{\mathrm{DD}}(s)= \\
& \frac{s \cdot\left[\left(R_{1} \tau_{2}+R_{1}^{\prime} \tau_{2}^{\prime}\right)+s \cdot\left(R_{1} \tau_{2}\left(\tau_{1}^{\prime}+\tau_{2}^{\prime}\right)+R_{1}^{\prime} \tau_{2}^{\prime}\left(\tau_{1}+\tau_{2}\right)\right)\right]}{s^{2}\left[R_{1} \tau_{2}\left(\tau_{1}^{\prime}+\tau_{2}^{\prime}\right)+R_{1}^{\prime} \tau_{2}^{\prime}\left(\tau_{1}+\tau_{2}\right)\right]+s\left[R_{1} \tau_{2}+R_{1}\left(\tau_{1}^{\prime}+\tau_{2}^{\prime}\right)+R_{1}^{\prime}\left(\tau_{1}+\tau_{2}\right)+R_{1}^{\prime} \tau_{2}^{\prime}\right]+\left[R_{1}+R_{1}^{\prime}\right]} .
\end{aligned}
$$

En esta ecuación se observa que el circuito presenta 2 polos y 2 ceros. Uno de estos últimos está en el origen y otorga a la red su característica de acoplamiento AC (ganancia nula en DC).

\subsubsection{Sensibilidad a los desapareamientos.}

Si se cumple la condición

$$
\tau_{2}=\tau_{2}^{\prime}
$$

La ecuación (6.13) se transforma en:

$$
G_{D D}(s)=\frac{s \cdot\left[\tau_{2}\left(R_{1}+R_{1}^{\prime}\right)+s \cdot\left(R_{1} \tau_{2}\left(\tau_{1}^{\prime}+\tau_{2}\right)+R_{1}^{\prime} \tau_{2}\left(\tau_{1}+\tau_{2}\right)\right)\right]}{s^{2}\left[R_{1} \tau_{2}\left(\tau_{1}^{\prime}+\tau_{2}\right)+R_{1}^{\prime} \tau_{2}\left(\tau_{1}+\tau_{2}\right)\right]+s\left[R_{1} \tau_{2}+R_{1}\left(\tau_{1}^{\prime}+\tau_{2}\right)+R_{1}^{\prime}\left(\tau_{1}+\tau_{2}\right)+R_{1}^{\prime} \tau_{2}\right]+\left[R_{1}+R_{1}^{\prime}\right]},
$$


que luego de algo de álgebra se puede llevar a:

$$
G_{\mathrm{DD}}(s)=\frac{s \cdot\left[s+\frac{R_{1}+R_{1}^{\prime}}{R_{1}\left(\tau_{1}^{\prime}+\tau_{2}\right)+R_{1}^{\prime}\left(\tau_{1}+\tau_{2}\right)}\right]}{s^{2}+s \cdot\left[\frac{1}{\tau_{2}}+\frac{R_{1}+R_{1}^{\prime}}{R_{1}\left(\tau_{1}^{\prime}+\tau_{2}\right)+R_{1}^{\prime}\left(\tau_{1}+\tau_{2}\right)}\right]+\left[\frac{1}{\tau_{2}} \cdot \frac{R_{1}+R_{1}^{\prime}}{R_{1}\left(\tau_{1}^{\prime}+\tau_{2}\right)+R_{1}^{\prime}\left(\tau_{1}+\tau_{2}\right)}\right]} .
$$

Factorizando el denominador de (6.16) resulta:

$$
G_{\mathrm{DD}}(s)=\frac{s \cdot\left(s+\frac{R_{1}+R_{1}^{\prime}}{R_{1}\left(\tau_{1}^{\prime}+\tau_{2}\right)+R_{1}^{\prime}\left(\tau_{1}+\tau_{2}\right)}\right)}{\left(s+\frac{1}{\tau_{2}}\right) \cdot\left(s+\frac{R_{1}+R_{1}^{\prime}}{R_{1}\left(\tau_{1}^{\prime}+\tau_{2}\right)+R_{1}^{\prime}\left(\tau_{1}+\tau_{2}\right)}\right)}
$$

donde se puede observar que, en estas condiciones $\left(\tau_{2}=\tau_{2}^{\prime}\right)$, uno de los polos se cancela exactamente con un cero y la transferencia $G_{\mathrm{DD}}$ se reduce a:

$$
G_{D D}(s)=\frac{s}{s+1 / \tau_{2}} \quad .
$$

Es importante notar que la cancelación polo-cero no depende de los valores de $R_{1}$ y $R_{1}{ }_{1}$, sino únicamente de la condición $\tau_{2}=\tau_{2}^{\prime}$. Si esta condición no se verifica, la función transferencia $G_{\mathrm{DD}}(s)$ presentará dos ceros (uno de ellos en el origen) y dos polos:

$$
G_{\mathrm{DD}}(s)=\frac{s \cdot\left(s+z_{1}\right)}{\left(s+p_{1}\right)\left(s+p_{2}\right)}
$$

Si se considera un apareamiento perfecto entre los componentes, las singularidades en (6.19) adoptarán sus valores "nominales" dados por:

$$
z_{1 \mathrm{n}}=\frac{1}{\tau_{1}+\tau_{2}} \quad ; \quad p_{1 \mathrm{n}}=\frac{1}{\tau_{1}+\tau_{2}} \quad ; \quad p_{2 \mathrm{n}}=\frac{1}{\tau_{2}}
$$

Si se acepta ahora que existen tolerancias en los elementos, la cancelación $z_{1}-p_{1}$ no se produce en forma exacta (sus valores se apartarán de los nominales) y $G_{\mathrm{DD}}(s)$ estará dada por la expresión (6.13) que verifica la forma:

siendo:

$$
G_{\mathrm{DD}}(s)=\frac{s \cdot\left(b_{0}+s a_{0}\right)}{s^{2} a_{0}+a_{1} s+a_{2}}
$$

$$
\begin{aligned}
& b_{0}=R_{1} \tau_{2}+R_{1}^{\prime} \tau_{2}^{\prime} \\
& a_{0}=R_{1} \tau_{2}\left(\tau_{1}^{\prime}+\tau_{2}^{\prime}\right)+R_{1}^{\prime} \tau_{2}^{\prime}\left(\tau_{1}+\tau_{2}\right) \\
& a_{1}=R_{1} \tau_{2}+R_{1}\left(\tau_{1}^{\prime}+\tau_{2}^{\prime}\right)+R_{1}^{\prime}\left(\tau_{1}+\tau_{2}\right)+R_{1}^{\prime} \tau^{\prime} \\
& a_{2}=R_{1}+R_{1}^{\prime}
\end{aligned}
$$


Ubicación de los polos.

El denominador de (6.21) es:

$$
D(s)=s^{2} a_{0}+a_{1} s+a_{2}
$$

La variación en la posición de sus raíces (los polos $p_{1}$ y $p_{2}$ ) debido a desplazamientos en los valores de los coeficientes, en una aproximación de primer orden, están dadas por (ver APÉNDICE 12);

$$
\begin{aligned}
& \Delta p_{1}=\frac{-1}{a_{0}\left(p_{2}-p_{1}\right)}\left[p_{1}^{2} \Delta a_{0}+p_{1} \Delta a_{1}+\Delta a_{2}\right] \\
& \Delta p_{2}=\frac{-1}{a_{0}\left(p_{2}-p_{1}\right)}\left[p_{2}^{2} \Delta a_{0}+p_{2} \Delta a_{1}+\Delta a_{2}\right]
\end{aligned}
$$

Para estimar la variación en la ubicación de los polos se supondrá que $R^{\prime}{ }_{1}, R_{2}{ }_{2}$ y $C^{\prime}$ presentan desplazamientos incrementales respecto de $R_{1}, R_{2}$ y $C$, es decir:

$$
R_{1}^{\prime}=R_{1}+\Delta R_{1} \quad ; \quad R_{2}^{\prime}=R_{2}+\Delta R_{2} \quad ; \quad C^{\prime}=C+\Delta C
$$

Para expresar las variaciones en los coeficientes de $D(\mathrm{~s})$ a partir de estos incrementos, es necesario diferenciar (6.22), obteniendo:

$$
\Delta a_{i}=\frac{\partial a_{i}}{\partial R_{1}^{\prime}} \Delta R_{1}+\frac{\partial a_{i}}{\partial R_{2}^{\prime}} \Delta R_{2}+\frac{\partial a_{i}}{\partial C^{\prime}} \Delta C
$$

Calculando, para cada coeficiente, las derivadas parciales a partir de (6.22) y reemplazando esto en (6.27) se obtiene:

$$
\begin{aligned}
& \Delta a_{0}=\tau_{2}\left(\tau_{2}+2 \tau_{1}\right) \Delta R_{1}+\tau_{1}\left(\tau_{1}+2 \tau_{2}\right) \Delta R_{2}+2 R_{1} R_{2}\left(\tau_{1}+\tau_{2}\right) \Delta C \\
& \Delta a_{1}=2\left(\tau_{1}+\tau_{2}\right) \Delta R_{1}+2 \tau_{1} \Delta R_{2}+\left(R_{1}^{2}+2 R_{1} R_{2}\right) \Delta C \\
& \Delta a_{1}=\Delta R_{1}
\end{aligned}
$$

Reemplazando (6.28) en (6.24) y (6.25) y luego de bastante álgebra se llega a:

$$
\begin{gathered}
\Delta p_{1}=\frac{-1}{2 R_{1} \tau_{1}\left(\tau_{1}+\tau_{2}\right)^{2}}\left[-\tau_{1}^{2} \Delta R_{1}-\tau_{1}^{2} \Delta R_{2}-R_{1}^{2}\left(\tau_{1}+\tau_{2}\right) \Delta C\right] \\
\Delta p_{2}=\frac{-1}{2 R_{1} \tau_{1} \tau_{2}^{2}}\left[\tau_{1}^{2} \Delta R_{2}+\left(2 R_{1} R_{2} \tau_{1}-R_{1}^{2} \tau_{2}\right) \Delta C\right] .
\end{gathered}
$$


Ubicación de los ceros.

El numerador de (6.21) es:

$$
N(s)=s\left(b_{0}+s a_{0}\right) \quad \text {. }
$$

Este polinomio presenta una raíz en $s=0$ y la restante, es decir el cero real de la función transferencia, se produce en:

$$
z_{1}=-b_{0} / a_{0}
$$

La variación en la ubicación de esta singularidad debida a desplazamientos en los coeficientes de $N(\mathrm{~s})$, siempre dentro de una aproximación de primer orden (APÉNDICE 12) ,esta dada por:

$$
\Delta z_{1}=\frac{b_{0}}{a_{0}^{2}} \Delta a_{0}-\frac{1}{a_{0}} \Delta b_{0}
$$

Siguiendo el mismo procedimiento que en el punto previo, se obtiene:

$$
\Delta z_{1}=\frac{-1}{2 \tau_{1} \tau_{2}\left(R_{1}+R_{2}\right)} \frac{1}{\tau_{1}+\tau_{2}}\left[-\tau_{1} \tau_{2} \Delta R_{1}-\tau_{1} \tau_{2} \Delta R_{2}-R_{1} R_{2}\left(\tau_{1}+\tau_{2}\right) \Delta C\right]
$$

Cancelación polo cero.

Adoptando $R_{1}=R_{2}=R$, es decir $\tau_{1}=\tau_{2}=\tau$ las ecuaciones (6.29), (6.30) y (6.34) se transforman en :

$$
\begin{aligned}
& \Delta p_{1}=\frac{-p_{1}}{4}\left[\frac{\Delta R_{1}}{R}+\frac{\Delta R_{2}}{R}+2 \frac{\Delta C}{C}\right], \\
& \Delta p_{2}=\frac{-p_{2}}{2}\left[\frac{\Delta R_{2}}{R}+\frac{\Delta C}{C}\right], \\
& \Delta z_{1}=\frac{-z_{1}}{4}\left[\frac{\Delta R_{1}}{R}+\frac{\Delta R_{2}}{R}+2 \frac{\Delta C}{C}\right],
\end{aligned}
$$

donde se puede observar claramente que $\Delta p_{1}=\Delta z_{1}$. La peor situación se produce cuando las variaciones en $R_{1}$ y $R_{2}$ tienen igual signo. Suponiendo igual dispersión relativa para ambos resistores, los desplazamientos resultan ${ }^{2}$ :

$$
\frac{\Delta p_{1}}{p_{1}}=-\frac{1}{2}\left[\frac{\Delta R}{R}+\frac{\Delta C}{C}\right] ; \quad \frac{\Delta p_{2}}{p_{2}}=-\frac{1}{2}\left[\frac{\Delta R}{R}+\frac{\Delta C}{C}\right] ; \quad \frac{\Delta z_{1}}{z_{1}}=-\frac{1}{2}\left[\frac{\Delta R}{R}+\frac{\Delta C}{C}\right]
$$

\footnotetext{
${ }^{2}$ Estas expresiones son tan simples que no honran el esfuerzo invertido en su cálculo. Es muy probable que exista un camino más simple y elegante para llegar a ellas.
} 
Las ubicaciones de las singularidades, expresadas a partir de los desbalances $\Delta R$ y $\Delta C$ entre los elementos resultan:

$$
\begin{aligned}
& z_{1}=\frac{1}{\tau_{1}+\tau_{2}}\left[1-\frac{1}{2}\left(\frac{\Delta R}{R}+\frac{\Delta C}{C}\right)\right] ; \\
& p_{1}=\frac{1}{\tau_{1}+\tau_{2}}\left[1-\frac{1}{2}\left(\frac{\Delta R}{R}+\frac{\Delta C}{C}\right)\right] ; \\
& p_{2}=\frac{1}{\tau_{2}}\left[1-\frac{1}{2}\left(\frac{\Delta R}{R}+\frac{\Delta C}{C}\right)\right] .
\end{aligned}
$$

Por ejemplo, si $R_{1}=R_{2}=5 \mathrm{M} \Omega$ y $C=1 \mu \mathrm{F}$, las singularidades son:

$$
z_{1}=-0.1000 \quad ; \quad p_{1}=-0.1000 \quad ; \quad p_{2}=-0.2000
$$

Considerando desplazamientos del $5 \%$ en los componentes, esto es: $R_{1}{ }_{1}=5.25 \mathrm{M} \Omega$, $R_{2}{ }_{2}=5.25 \mathrm{M} \Omega$ y $C^{\prime}=1.05 \mu \mathrm{F}$, el cero y los polos se trasladan a:

$$
z_{1}=-0.0955 \quad ; \quad p_{1}=-0.0950 \quad ; \quad p_{2}=-0.1909
$$

Se observa que tal como predice (6.39), el desplazamiento en $z_{1}, p_{1}, p_{2}$ es de alrededor del $5 \%$, mientras que el apartamiento entre $p_{1}$ y $z_{1}$ es menor del $0.5 \%$, es decir que la cancelación es muy aproximada.

\section{Distorsión en la respuesta transitoria.}

En aplicaciones biomédicas es muy importante que el A.B. presente una adecuada respuesta transitoria y son muy exigentes las especificaciones al respecto (AAMI, 1998).

Como se vio anteriormente, si no se produce la cancelación polo-cero en forma exacta, el circuito presentará dos ceros y dos polos. Su función transferencia adoptará la forma:

$$
G_{\mathrm{DD}}(s)=\frac{s \cdot\left(s+z_{1}\right)}{\left(s+p_{1}\right)\left(s+p_{2}\right)}
$$

La respuesta de este sistema a un escalón de entrada está dada por:

$$
r(t)=\frac{\left(z_{1}-p_{2}\right)}{\left(p_{1}-p_{2}\right)} e^{p_{2} \cdot t}+\frac{\left(z_{1}-p_{1}\right)}{\left(p_{2}-p_{1}\right)} e^{p_{1} \cdot t} \quad .
$$

Si se considera el caso $\tau_{1}=\tau_{2}=\tau$, es $\operatorname{decir} p_{1}=-1 / 2 \tau$ y $p_{2}=-1 / \tau$ resulta:

$$
r(t)=1 \cdot e^{-t / \tau}-2 \tau\left(z_{1}-p_{1}\right) e^{-t /(2 \tau)}
$$


En algunas aplicaciones, la constante de tiempo $\tau$ puede llegar a ser de varios segundos; pero aún en estas condiciones el aporte del segundo término, relacionado con el polo $p_{2}$ es poco notable. Retomando el ejemplo anterior $\left(R_{1}=R_{2}=5 \mathrm{M} \Omega, C=1 \mu \mathrm{F}\right.$ y desbalances del 5\%), resultaría:

$$
r(t)=1 \cdot e^{-t / 10}-0.01 \cdot e^{-t / 20},
$$

y para este caso típico, la amplitud del residuo asociado a $p_{1}$ (el polo no deseado) resulta dos órdenes de magnitud por debajo del correspondiente a $p_{2}$.

\subsubsection{Factores de Mérito.}

El circuito propuesto es totalmente pasivo. Para evaluar sus características y poder compararlas con otras opciones, en los puntos siguientes se considerará que se utiliza en conjunto con un Amplificador de Instrumentación F-D de dos A.O., componiendo un A.B. completo según se muestra en la Fig.6.9.

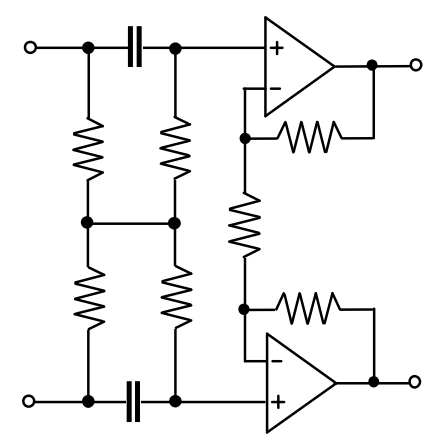

Figura 6.9. A.B. que utiliza la red de acoplamiento propuesta.

\section{Máxima tensión diferencial de entrada de DC V V.DC.MAx admisible}

La componente de DC de modo diferencial $V_{\text {iDC.MAX }}$ que este circuito admite en su entrada no está limitada, dado que su entrada se encuentra acoplada capacitivamente para este tipo de señal.

$$
V_{\mathrm{iDC} \cdot \mathrm{MAX}}=\infty
$$

\section{Tensión de Offset a la Salida.}

La tensión de offset en la salida de este A.B. depende exclusivamente del I.A. La misma está dada por la diferencia $\Delta V_{\text {OFFSET.AO }}$ entre las tensiones de offset de cada uno de los A.O., amplificada por la ganancia total $G_{\mathrm{DD}}$.

$$
V_{\text {o.OFFSET }}=\Delta V_{\text {OFFSET.AO }} \cdot G_{\mathrm{DD}} \quad .
$$


Este esquema exige A.O. con baja corriente de offset ( $i_{\text {OFFSET }}$, pues las diferencias de potencial que estas produzcan sobre $R_{2}, R_{2}^{\prime}\left(i_{\mathrm{OFFSET}} \cdot 2 R_{2}\right)$ también serán amplificadas por la ganancia diferencial $G_{\mathrm{DD}}$ del A.B. Si se toma este cuidado, utilizando amplificadores de tecnología CMOS o JFET, la tensión de DC a la salida debida a este efecto no es significativa.

\section{Rechazo de Modo Común.}

La red de acoplamiento propuesta presenta un $C_{M R R_{D P}}$ infinito. El rechazo total $\mathrm{CMRR}_{\mathrm{T}}$ del A.B. completo resulta limitado por el correspondiente al I.A. y está dado por:

$$
\frac{1}{C M R R_{\mathrm{T}}} \cong \frac{1}{C M R R_{\mathrm{A} 1}}-\frac{1}{C M R R_{\mathrm{A} 2}}
$$

siendo $C M R R_{\mathrm{A} 1}$ y $C M R R_{\mathrm{A} 2}$ los rechazos de modo común de cada uno de los A.O que componen el amplificador.

\section{Tensión de Ruido Equivalente a la Entrada}

El ruido presente a la salida del A.B. de la Fig.6.9. se debe principalmente a los A.O. Si bien los resistores de la red de acoplamiento son de valor elevado, en el rango de frecuencias de interés se encuentran "cortocicuitados" por las impedancias de electrodo (ver APÉNDICE 13) y no aportan significativamente al ruido total. La tensión de ruido referida a la entrada $e_{\mathrm{i} . \mathrm{T}}$ resulta:

$$
e_{i . T} \cong \sqrt{2} \cdot e_{A O}
$$

siendo $e_{\mathrm{AO}}$ la tensión de ruido de cada uno de los A.O.

\subsubsection{Resultados Experimentales.}

Para verificar el funcionamiento del circuito se construyó un prototipo de A.B. utilizando la red propuesta con $C=1 \mathrm{uF}, R_{1}=R_{2}=4.7 \mathrm{M} \Omega$ y como I.A. un amplificador de instrumentación integrado INA111. El CMRR a $50 \mathrm{~Hz}$ resultó de $112 \mathrm{~dB}$ y la impedancia de entrada de modo común de $5 \mathrm{pF}$. Estos valores se corresponden muy aproximadamente al CMRR y a la impedancia $Z_{C}$ propia del I.A. que fueron determinados en forma previa a la inclusión de la red de acoplamiento. La red de entrada provee el deseado acoplamiento en $\mathrm{AC}$, sin degradar las características propias del I.A. 


\section{Esquema Activo. (Supresión de DC)}

El esquema general de un sistema de acoplamiento en AC activo, también conocido como "supresión de DC", se muestra en la Fig.6.10. Esta estrategia se basa en realimentar en forma negativa las componentes de baja frecuencia, que de esta manera serán rechazadas.

Es habitual utilizar un integrador en la realimentación. Así, la ganancia de lazo abierto en DC es infinita y la ganancia de lazo cerrado resulta nula para este tipo de señal

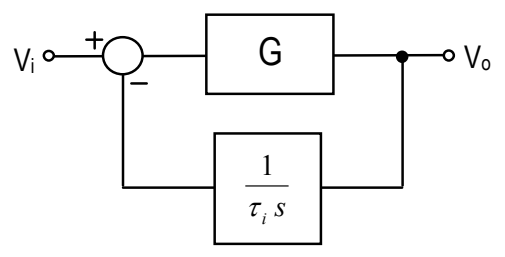

Figura 6.10. Esquema general del Acoplamiento en AC Activo (Supresión de DC)

A partir del diagrama de bloques de la Fig.6.10 se obtiene la siguiente función de transferencia de lazo cerrado:

$$
T(s)=\frac{s \cdot \tau_{i}}{1+s \cdot \tau_{i} / G}
$$

que verifica una respuesta pasa-altos de primer orden.

Esta estrategia presenta una ventaja importante: si el A.B. se satura (por ejemplo debido a una conexión-desconexión de un electrodo), es posible recuperar rápidamente el nivel base reduciendo momentáneamente la constante de tiempo $\tau_{\mathrm{i}}$; algo que de otra manera podría demandar varios segundos. Esta función se conoce como "DC restoration" ó "recuperación rápida" y resulta sumamente útil en aplicaciones biomédicas, fundamentalmente en aquellos equipos sometidos a perturbaciones gran amplitud; como "artefactos de movimiento"3 (motion artifacts) (GRIMBERGEN, 1995). Estas perturbaciones son muy frecuentes en aquellos estudios que se realizan con el paciente en movimiento (ergometrías) o realizando sus actividades cotidianas (Holter) y en estos caso resulta muy deseable disponer de un mecanismo de recuperación.

\subsection{El Circuito Clásico.}

Un circuito clásico de un A.B., que implementa el concepto de "supresión de DC", se muestra en la Fig.6.11 (SMIT, 1987). Como puede observarse, presenta alta impedancia de entrada de modo común $\mathrm{Z}_{\mathrm{C}}$ siendo apto para aplicaciones biomédicas.

\footnotetext{
${ }^{3}$ Estas perturbaciones, en general de baja frecuencia, se producen cuando el paciente realiza algún movimiento que altera la interfase electrodo piel.
} 


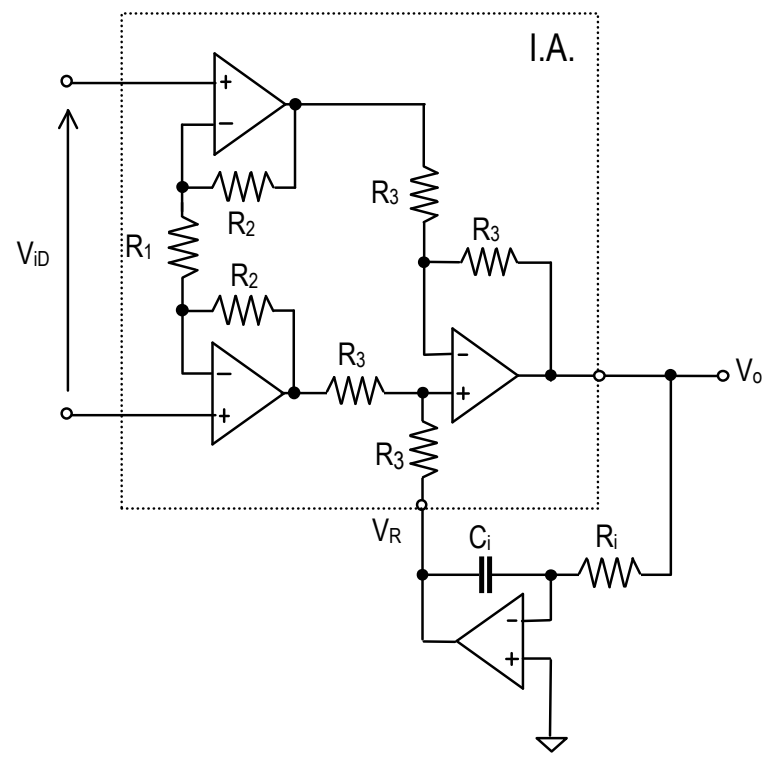

Figura 6.11. Circuito "Clásico" de un Amplificador para Biopotenciales con acoplamiento activo.

El circuito consiste en un amplificador de instrumentación y un integrador. El I.A de tres A.O. posee dos entradas: una de tipo diferencial $V_{\mathrm{iD}}$ y otra single ended $V_{\mathrm{R}}$. Su salida depende de ellas según:

$$
V_{o}=G_{\mathrm{DD}} \cdot V_{\mathrm{iD}}+V_{\mathrm{R}} \quad ; \quad G_{\mathrm{DD}}=1+2 R_{2} / R_{1}
$$

Entonces, si $V_{\mathrm{R}}$ se conecta a la integral de la tensión de salida, es decir:

$$
V_{\mathrm{R}}=-\frac{V_{o}}{s \cdot R_{\mathrm{i}} C_{\mathrm{i}}}
$$

Reemplazando (6.52) en (6.51) la transferencia global del circuito resulta:

$$
\frac{V_{\mathrm{o}}}{V_{\mathrm{iD}}}=\frac{G_{\mathrm{DD}} \cdot s \tau_{i}}{1+s \tau_{i}} \quad ; \quad \tau_{\mathrm{i}}=R_{\mathrm{i}} \cdot C_{\mathrm{i}} \quad,
$$

verificando una respuesta de tipo pasa-altos de primer orden.

\subsubsection{Factores de Mérito.}

Máxima tensión diferencial de entrada de DC V Vi.DC.MAx admisible.

La máxima tensión admisible de DC a la entrada del A.B. ( $\left.V_{\text {i.DC.MAX }}\right)$, se alcanza cuando la salida del integrador $\left(V_{\mathrm{R}}\right)$ iguala a la tensión de alimentación $V_{\mathrm{CC}}$. En esta condición, considerando que el sistema consigue rechazar totalmente la componente de DC (esto es $V_{\mathrm{o}}=0$ ), la ecuación (6.51) se transforma en:

$$
0=G_{\mathrm{DD}} \cdot V_{\mathrm{i} . \mathrm{DC} . \mathrm{MAX}}-V_{\mathrm{CC}},
$$


de donde se obtiene que $V_{\text {i.DC.MAX }}$ está dada por:

$$
V_{\text {i.DC.MAX }}=\frac{V_{\mathrm{CC}}}{G_{\mathrm{DD}}} .
$$

\section{Tensión de Offset a la salida.}

La tensión de offset a la salida $V_{\text {o.DC }}$ se debe exclusivamente a la tensión de offset del A.O. que implementa el integrador:

$$
V_{\text {o.DC }}=V_{\text {OFFSET.AO }} \cdot
$$

\section{Ruido Equivalente a la Entrada.}

El sistema de supresión de DC no incrementa significativamente ${ }^{4}$ el ruido del A.B. que sigue siendo el correspondiente al I.A. de tres A.O., es decir:

$$
e_{i . T} \cong \sqrt{2} \cdot e_{\mathrm{AO}} \text {. }
$$

Donde $e_{\mathrm{AO}}$ representa la tensión de ruido equivalente correspondiente a un A.O.

\section{Rechazo de Modo Común.}

Si la impedancia de salida del integrador es despreciable frente a $R_{3}$ (ver Fig.6.11), la realimentación a $V_{\mathrm{R}}$ no degrada el CMRR del I.A. El mismo queda dado por:

$$
C M R R_{\mathrm{T}}^{-1}=\left(1 / C_{\mathrm{AO} 2}-1 / C_{\mathrm{AO} 1}\right)+\frac{2 \cdot t}{G_{\mathrm{DD}}}
$$

siendo $t$ la tolerancia de los resistores. Como la ganancia $G_{\mathrm{DD}}$ está limitada a valores moderados, el CMRR queda dominado por el segundo término, resultando:

$$
C M R R_{\mathrm{T}} \cong \frac{G_{\mathrm{DD}}}{2 \cdot t}
$$

La máxima ganancia posible está relacionada a través de (6.55) con la tensión diferencial de DC $V_{\text {iD.MAx }}$ que el A.B. debe admitir a su entrada. Utilizando esta vinculación, el CMRR puede expresarse como:

$$
C M R R_{\mathrm{T}}=\frac{V_{\mathrm{CC}}}{V_{\mathrm{iDC} . \mathrm{MAX}} \cdot 2 t}
$$

Considerando $V_{\mathrm{CC}}= \pm 15 \mathrm{~V}, V_{\mathrm{iD} . \mathrm{MAX}}= \pm 300 \mathrm{mV}$ y resistores al $1 \%$, resulta un CMRR de $68 \mathrm{~dB}$ que se podría calificar de marginal. En una situación más "actual" con el mismo rango de $V_{\mathrm{iDC}} \mathrm{y}$ una tensión de alimentación $V_{\mathrm{CC}}= \pm 1.5 \mathrm{~V}$, el CMRR sería de solo $48 \mathrm{~dB}$, que no es aceptable.

\footnotetext{
${ }^{4}$ La resistencia $R_{\mathrm{i}}$ del integrador contribuye con ruido pero solo para frecuencias por debajo de la frecuencia de corte.
} 


\section{Principales Inconvenientes de esta Topología..}

De acuerdo a (6.55), para que el A.B admita un buen rango de tensiones de DC de entrada, se requiere una tensión de alimentación elevada y/o una baja ganancia: dos condiciones desagradables. La primera, va en contra de la tendencia actual de alimentar con baterías de reducida tensión $(3 \mathrm{~V})$ y la segunda dificulta conseguir altos CMRR y bajos niveles de ruido.

Por ejemplo, si se dispone de $V_{\mathrm{CC}}= \pm 15 \mathrm{~V}$ y se requiere una $V_{\text {i.DC.MAX }}=300 \mathrm{mV}$ según exige la norma AAMI (AAMMI, 1998), la máxima ganancia admisible resulta de 50 veces $(33 \mathrm{~dB})$. Esto es aceptable y permite conseguir CMRR razonablemente altos y bajos niveles de ruido (miles de equipos comerciales funcionando en estas condiciones avalan esta afirmación). Ahora, si se considera una aplicación más "moderna" con una tensión de alimentación de $3 \mathrm{~V}$ (esto es equivalente a $V_{\mathrm{CC}}= \pm 1.5 \mathrm{~V}$ ), la máxima ganancia admisible es de solo 5 veces, lo cual hace muy difícil obtener altos CMRR y surge como imperativo aportar nuevas soluciones a este problema. Además, el hecho de disponer de una ganancia reducida en la primer etapa, exige agregar etapas adicionales de amplificación lo cual incrementa el consumo del amplificador.

El principal inconveniente del esquema clásico radica en que su primer etapa amplifica tanto la señal de interés como las componentes de DC producto de los potenciales de electrodo. Para evitar esto, es necesario suprimir las componentes de baja frecuencia antes que ingresen al amplificador, es decir ubicar el punto de resta de la Fig.6.10. directamente a la entrada del A.B., en forma previa a cualquier amplificación.

\subsection{Una solución propuesta : Circuito F-D con supresión de DC.}

En esta tesis se propone una estrategia que implementa el esquema de la Fig.6.10. respetando una estructura F-D. Todas sus conexiones a masa se realizan a través de generadores de tensión cuyo valor es idéntico a la tensión de modo común de entrada

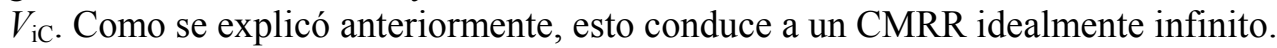

Una topología F-D que permite implementar la estructura de supresión de DC de la Fig.6.10 se propone ${ }^{5}$ en la Fig.6.12. Este circuito por ser F-D, tiene un muy buen rango de entrada y resulta particularmente apropiado para A.B. alimentados mediante una fuente simple. Además, su salida diferencial puede conectarse directamente a convertidores ADC de entrada diferencial.

\footnotetext{
${ }^{5}$ En realidad este simple "se propone" oculta decenas de circuitos que se ensayaron buscando la solución al problema, muchos de ellos no resistieron el análisis en papel, otros se derrumbaron recién en las simulaciones y unos pocos (los más perversos) recién mostraron sus falencias al ser construidos. El circuito de la Fig.6.12 fue el único que sobrevivió a todas las etapas. Esto muestra lo poco sistemático que puede ser el diseño de circuitos analógicos.
} 


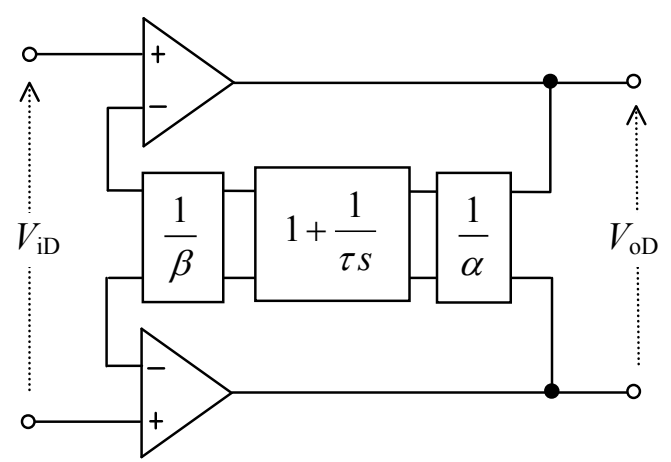

Figura 6.12. Esquema del circuito F-D propuesto para implementar el acoplamiento en AC activo.

\subsubsection{Análisis del circuito}

El circuito consiste en un amplificador F-D con una red de realimentación también F-D. Esta última contiene un integrador, el cual asegura un rechazo infinito a las componentes de DC.

Si se consideran A.O. con ganancia infinita, lo cual implica una diferencia de potencial nula entre sus entradas, la relación entre la tensión diferencial de entrada y la tensión diferencial de salida está dada por:

$$
V_{\mathrm{iD}}=V_{\mathrm{oD}} \cdot \frac{1}{\alpha} \cdot\left(1+\frac{1}{s \tau}\right) \cdot \frac{1}{\beta}
$$

De esta expresión puede obtenerse la ganancia diferencial $G_{\mathrm{DD}}(s)$ como:

$$
G_{\mathrm{DD}}(s)=\frac{V_{\mathrm{oD}}}{V_{\mathrm{iD}}}=\frac{\alpha \beta s \tau}{1+s \tau}
$$

que verifica una respuesta pasa-altos de primer orden. Su ganancia a frecuencias medias $G_{\mathrm{DD}}$ y su frecuencia de corte inferior $f_{\mathrm{L}}$ están dadas por:

$$
\begin{aligned}
& G_{\mathrm{DD}}=\alpha \beta \\
& f_{\mathrm{L}}=\frac{1}{2 \pi \tau}
\end{aligned}
$$




\subsubsection{Implementación de la estrategia.}

La estructura propuesta puede implementarse mediante el circuito de la Fig.6.13.

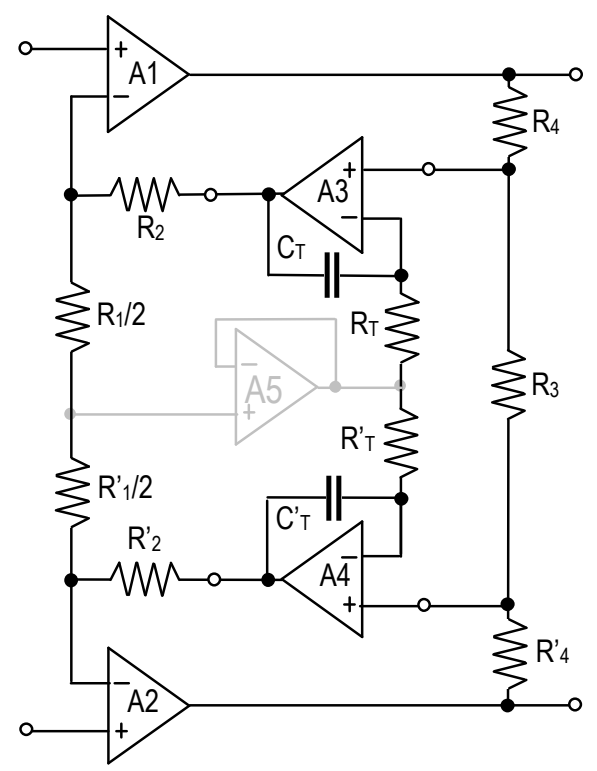

Figura 6.13. Circuito práctico que implementa la estrategia propuesta para acoplamiento en AC activo.

Si se acepta ${ }^{6}$ inicialmente $R_{1}=R^{\prime}{ }_{1}, R_{2}=R^{\prime}{ }_{2}, R_{4}=R_{4}^{\prime}, R_{\mathrm{T}}=R^{\prime}$, and $C_{\mathrm{T}}=C^{\prime}{ }_{\mathrm{T}}$, los parámetros $\alpha, \beta$ y $\tau$ definidos anteriormente están relacionados con los elementos del circuito mediante:

$$
\begin{aligned}
& \alpha=1+2 R_{4} / R_{3} \\
& \beta=1+2 R_{2} / R_{1} \\
& \tau=R_{\mathrm{T}} C_{\mathrm{T}}
\end{aligned}
$$

Para tensiones de modo diferencial, este circuito se comporta según la función de transferencia (6.62). Si se aplica una tensión de entrada de modo común, no circulará corriente alguna sobre los resistores $R_{1} / 2, R^{\prime}{ }_{1} / 2$ (ambos extremos de estas resistencias se encuentran al potencial $V_{\text {iC }}$ ) y por lo tanto tampoco circularán corrientes sobre $R_{2}$, $R_{2}{ }_{2}$. Las salidas de $\mathrm{A} 3 \mathrm{y} \mathrm{A} 4$, que son generadores de tensión conectados a masa, presentarán una tensión igual a $V_{\mathrm{iC}}$, verificando la condición deseada para conseguir un CMRR infinito (ver APÉNDICE 5).

\footnotetext{
${ }^{6}$ De todos modos, como se mostrará más adelante el CMRR de este circuito es independiente del desbalance entre los componentes pasivos, siendo esta una de sus principales virtudes.
} 
Por otra parte, como la salida diferencial del integrador es nula, también lo será su entrada de modo diferencial, la cual coincide con la salida del amplificador. En cuanto a tensiones de modo común, la correspondiente a la entrada del integrador no está definida, quedando sin control y a merced de las corrientes de polarización de los A.O. Para fijar la tensión de modo común del integrador se incluye el A5 (indicado en gris en la Fig.6.13), que copia la tensión de modo común de entrada estableciendo el potencial de modo común. De este modo, todas las conexiones del circuito a masa continúan siendo realizadas a través de generadores de tensión iguales a la tensión de modo común de entrada $V_{\text {ic }}$.

\section{Consideraciones de estabilidad del Circuito.}

Utilizando A.O. internamente compensados, el circuito de la Fig.6.13 resulta estable para tensiones de modo diferencial, dado que la ganancia de realimentación para este tipo de señal $\left(1 / G_{\mathrm{DD}}\right)$, es mucho menor que la unidad. Sin embargo pueden surgir problemas de estabilidad para señales de modo común.

Este problema es muy similar al caso del amplificador de instrumentación F-D de dos A.O. analizado en (WhITE, 1987). Sucede que los atenuadores balanceados $\alpha$ y $\beta$ atenúan las señales de modo diferencial pero presentan ganancia unitaria para tensiones de modo común. El circuito tiene una realimentación débil para señales de modo diferencial pero una realimentación muy fuerte (unitaria) para señales de modo común.

Un modelo simple que permite analizar los problemas de estabilidad de modo común, válido para frecuencias cercanas a la frecuencia de ganancia unitaria $(0 \mathrm{~dB})$, se muestra en la Fig.6.14.

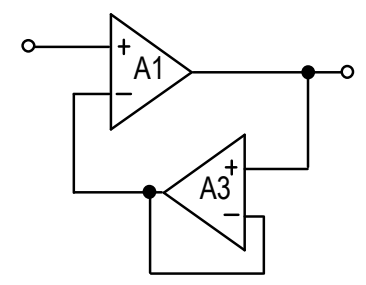

Figura 6.14. Circuito utilizado para analizar la estabilidad de modo común. Este esquema es válido para frecuencias cercanas al corte de $0 \mathrm{~dB}$.

Los amplificadores A1 y A3 aportan fase a la ganancia de lazo abierto y el circuito es potencialmente inestable. Para asegurar su estabilidad sin incluir componentes a masa $^{7}$, se utilizaron A.O. de mayor producto ganancia ancho de banda (GPB) en el integrador que en la etapa de entrada. De esta manera es posible asegurar que a la frecuencia para la cual ganancia de A1 es unitaria, la fase aportada por el seguidor implementado por A3 sea despreciable, asegurando así un Margen de Fase adecuado.

\footnotetext{
${ }^{7}$ Para introducir atenuación de modo común es indispensable incluir componentes a masa, lo cual degradaría el CMRR.
} 


\subsubsection{Factores de Mérito.}

\section{Rango de $D C$ de entrada.}

Considerando amplificadores operacionales cuyas salidas puedan operar desde la tensión de alimentación positiva $+V_{\mathrm{CC}}$ hasta la tensión de alimentación negativa $-V_{\mathrm{CC}}$ de una fuente de alimentación simétrica ${ }^{8}$ (salida rail-to-rail), la máxima tensión de DC admisible a la entrada se produce cuando el integrador F-D entrega su máxima excursión que es $2 V_{\mathrm{CC}}$ (una salida en $+V_{\mathrm{CC}}$ y la opuesta en $-V_{\mathrm{CC}}$ ). Dado que la salida del integrador se presenta a la entrada atenuada en un factor $\beta$, la máxima tensión de DC de entrada admisible $V_{\text {iD.MAX }}$ resulta:

$$
V_{\text {i.DC.MAX }}=2 V_{\mathrm{CC}} / \beta
$$

\section{Tensión de Offset a la salida.}

La tensión de offset a la salida se debe la diferencia entre las tensiones de offset individuales de los A.O. A3 y A4 que componen el integrador. Esta diferencia,

$$
\Delta V_{\text {OFFSET.AO }}=V_{\text {OFFSET.A3 }}-V_{\text {OFFSET.A }}
$$

producirá una tensión de DC a la salida $V_{\text {o.DC, }}$ dada por:

$$
V_{\text {O.DC }}=\alpha \cdot \Delta V_{\text {OFFSET.AO }},
$$

que puede expresarse en función de $\beta$ como:

$$
V_{\text {o.DC }}=\frac{G_{\mathrm{DD}}}{\beta} \Delta V_{\text {OFFSET.AO }} .
$$

Es decir que $V_{\text {i.DC.MAX }}$ es $\beta$ veces menor que la diferencia entre las tensiones de offset de los A.O. que componen la etapa de entrada.

\section{Ruido Equivalente a la Entrada.}

El ruido del amplificador propuesto se debe principalmente a los A.O. A1, A2 que componen la etapa de entrada. El ruido propio de $R_{1}$ y $R^{\prime}{ }_{1}$ también aparece directamente a la entrada, pero utilizando resistores de valor moderado, esta contribución es despreciable. El ruido de A3 y A4, aparece a la entrada atenuado en un factor $\beta$ y $\mathrm{A} 5$ contribuye solo con ruido de modo común. El ruido total referido a la entrada resulta:

$$
e_{\mathrm{i} . \mathrm{T}}^{2}=e_{\mathrm{A} 1}^{2}+e_{\mathrm{A} 2}^{2}+\left(e_{\mathrm{A} 3}^{2}+e_{\mathrm{A} 4}^{2}\right) / \beta^{2} .
$$

${ }^{8}$ Se considero aquí una fuente simétrica para facilitar la comparación con otras configuraciones, pero esta topología puede trabajar perfectamente a partir de una fuente simple.

${ }^{9} \mathrm{Un}$ resistor de $6 \mathrm{k} \Omega$ tiene un ruido propio del orden del correspondiente a un muy buen A.O. $(\approx 10 \mathrm{nV} / \sqrt{\mathrm{Hz}})$. 
Usualmente $\beta$ es suficientemente grande como para despreciar el segundo término y el ruido queda dominado por las contribuciones de A1 y A2. En estas condiciones, la tensión eficaz de ruido referida a la entrada $e_{\mathrm{i} . \mathrm{T}}$ se puede aproximar por:

$$
e_{i . T}=\sqrt{2} \cdot e_{\mathrm{AO}}
$$

Los resistores $R_{\mathrm{T}}$, que determinan la frecuencia de corte inferior $f_{\mathrm{L}}$, suelen resultar de valor elevado y también contribuyen al ruido total, pero su contribución no es importante dentro del rango de frecuencias de interés. Para frecuencias medias, los capacitores $C_{\mathrm{T}}, C^{\prime}$, pueden considerarse corto-circuitos y el ruido de $R_{\mathrm{T}}$ no tiene efecto alguno sobre el circuito. Este mecanismo, es similar al ruido incorporado por los resistores de polarización en un esquema de acoplamiento en AC pasivo (APÉNDICE 13).

\section{Rechazo de Modo Común.}

Dado que se trata de un circuito F-D cuyas conexiones a masa se realizan exclusivamente a través de generadores de tensión igual a la tensión de modo común de entrada, su CMRR será idealmente infinito. En realidad, al igual que en el amplificador F-D de dos A.O, el CMRR está limitado por el desbalance entre los CMRR de los A.O. que componen la etapa de entrada. Esto es:

$$
\frac{1}{C M R R_{T}} \cong \frac{1}{C M R R_{A 1}}-\frac{1}{C M R R_{A 2}}
$$

\section{Ecuaciones de Diseño.}

Como un ejemplo de diseño y para validar el análisis previo, se implementó un amplificador de ECG con las siguientes especificaciones: $V_{\mathrm{iDC} . \mathrm{MAX}}> \pm 500 \mathrm{mV}$, una frecuencia de corte $f_{\mathrm{L}}=0.05 \mathrm{~Hz}, V_{\mathrm{CC}}= \pm 2.5 \mathrm{~V}$ y una ganancia de modo diferencial $G_{\text {DD }}$ de aproximadamente 500 veces.

A partir del rango de DC de entrada deseado, utilizando la ecuación (6.66.a) se obtiene el valor de $\beta$ :

$$
\beta=\frac{2 V_{C C}}{V_{i d c}}=10
$$

Luego se calcula $\alpha$ para obtener la ganancia necesaria como:

$$
\alpha=\frac{G_{\mathrm{DD}}}{\beta}=50
$$

y por último, se dimensiona la constante de tiempo $R_{\mathrm{T}} C_{\mathrm{T}}$ para conseguir la frecuencia de corte deseada según:

$$
R_{\mathrm{T}} C_{\mathrm{T}}=\frac{1}{2 \pi f_{\mathrm{L}}}=3.18 \mathrm{~s}
$$


Finalmente, seleccionando componentes de valores estándar resultan: $R_{\mathrm{T}}=3.3 \mathrm{M} \Omega$, $C_{\mathrm{T}}=1 \mu \mathrm{F}, R_{1} / 2=910 \Omega, R_{2}=7.5 \mathrm{k} \Omega, R_{3}=2.2 \mathrm{k} \Omega$ y $R_{4}=56 \mathrm{k} \Omega$, que definen:

$$
\alpha=51.9, \beta=9.24, G_{\mathrm{DD}}=\alpha \beta=480 \text { y } f_{\mathrm{L}}=0.048 \mathrm{~Hz} \text {. }
$$

El circuito final, que se muestra en la Fig.6.15, se implementó utilizando A.O. TLV2454 para la etapa de entrada $(\mathrm{GBP}=220 \mathrm{kHz})$ y TLV2474 $(\mathrm{GBP}=2.8 \mathrm{MHz})$ para el integrador. Se incorporaron además las llaves $\mathrm{SW}_{\mathrm{FR}}$ y $\mathrm{SW}_{\mathrm{FR}}$ para permitir la recuperación rápida del amplificador frente a una condición de saturación. Esto se consigue operando estas llaves, cambiando de la $R_{\mathrm{T}}$ nominal que determina $\tau=3.3 \mathrm{~s} \mathrm{a}$ una centésima parte ( $\tau=33 \mathrm{~ms}$ ), consiguiendo así una rápida recuperación de la línea base.

El proceso de diseño para el amplificador propuesto es muy simple. Como se mostró con el ejemplo previo, consiste básicamente en la selección de los factores $\alpha$ y $\beta$. Para que el A.B. admita un elevado valor de DC de entrada $V_{\text {i.DC.MAX }} \beta$ deber ser pequeño (ver (6.67)), pero de acuerdo a (6.68) esto incrementa la contribución de ruido de A3 y A4. Entonces, la estrategia más conveniente para un diseño de bajo ruido es elegir el mas alto valor de $\beta$ compatible con la $V_{\text {iDC.MAX }}$ deseada y luego adoptar el valor de $\alpha$ necesario para conseguir la ganancia total.

Si se dispone de una $V_{\mathrm{CC}}$ de al menos $\pm 1.5 \mathrm{~V}(3 \mathrm{~V}), \beta=10$ provee un rango de DC de entrada de $\pm 300 \mathrm{mV}$, cumpliendo con el requerido por la norma AAMI (AAMI, 1998). Para este valor de $\beta$ la contribución de A3 y A4 al ruido equivalente de entrada no es significativa.

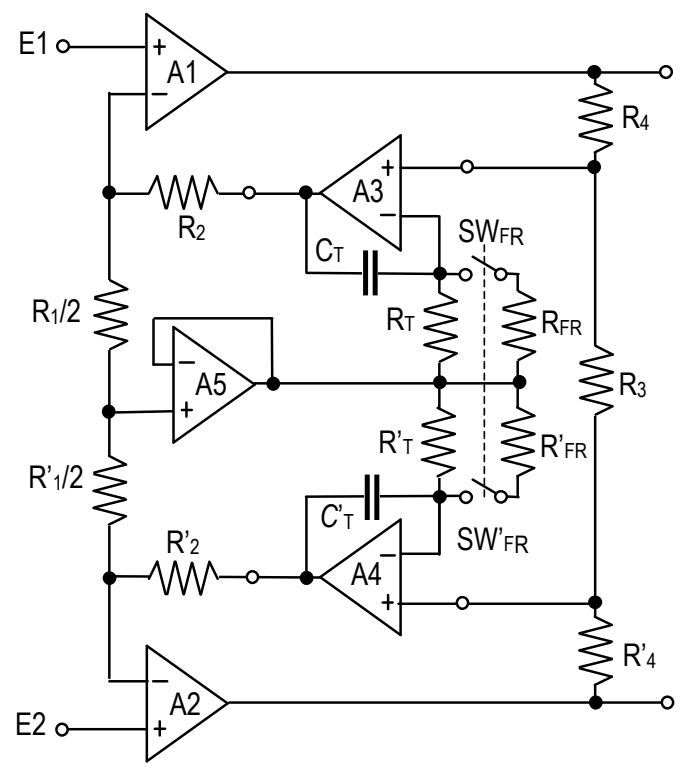

Figura 6.15. Circuito práctico para implementar la técnica propuesta. Se incluyen la llaves SWFR y SW'FR que permiten la recuperación rápida del amplificador luego de una situación de saturación. 


\subsubsection{Resultados Experimentales.}

Se construyó un prototipo utilizando resistores metal-film al $1 \%$ y capacitores multicapa al $10 \%$. El rechazo de modo común a $50 \mathrm{~Hz}$ del circuito resultó de $107 \mathrm{~dB}$ operando con una fuente simple de $5 \mathrm{~V}$ (equivalente a una fuente simétrica de $\pm 2.5 \mathrm{~V}$ ).

Para evaluar el comportamiento del sistema de "recuperación rápida" con señales reales, se implementó un amplificador ${ }^{10}$ de ECG basado en el circuito propuesto (SPINELLI, 2003).

En primer lugar se mantuvo la constante de tiempo "rápida"de $0.03 \mathrm{~s}$ por un tiempo prolongado simulando un reset manual. Esto corresponde al primer tramo grisado de la Fig.6.16, donde puede observarse claramente la distorsión de baja frecuencia. En el segundo tramo, las llaves se cerraron durante un pequeño intervalo, suficiente para recuperar la línea base, simulando un "reset automático". Esto suele implementarse al cambiar de derivación de acuerdo a lo recomendado en la sección 3.2.13.2 de la norma AAMI (AAMI, 1998).

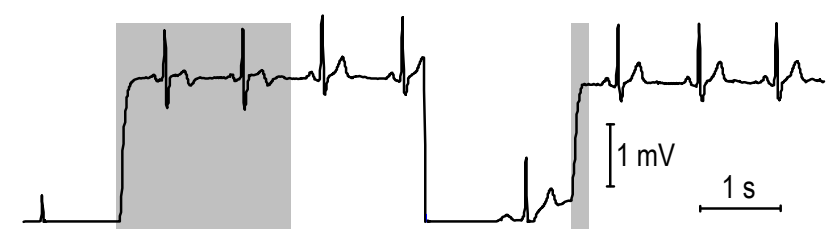

Figura 6.16. Registro de ECG obtenido con el A.B. construido. En las zonas grisadas se cerraron las llaves $S W_{F R}$ y $S W^{\prime} F R$ donde se observa que el amplificador recupera rápidamente el nivel base. En el primer tramo grisado también puede observarse la distorsión producida por la elevada frecuencia de corte inferior. En el segundo tramo las llaves se mantuvieron cerradas sólo el tiempo necesario para llevar al A.B. a un nivel de operación adecuado.

Este mecanismo de recuperación rápida, característica exclusiva del "acoplamiento activo", es muy útil en la práctica clínica porque permite que el registro alcance rápidamente el nivel base luego de una condición de saturación. Ésta puede producirse por diversos factores, por ejemplo debido a la selección de una nueva combinación de electrodos o por artefactos de movimiento. Por último, en la Fig.6.16 se muestra en detalle el registro de ECG obtenido donde no se observan signos de interferencia de la tensión de red.

\footnotetext{
${ }^{10} \mathrm{El}$ amplificador utilizado incorpora además un circuito reductor de modo común DRL que incrementa el CMRR unos $30 \mathrm{~dB}$ al mismo tiempo que asegura una apropiada tensión de modo común de entrada. Este circuito accesorio así también como el amplificador completo construido se analizarán más adelante en los CAPÍTULOS 7 y 8 respectivamente.
} 


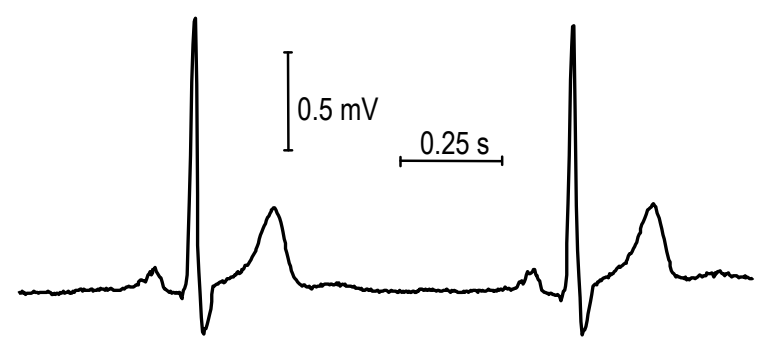

Figura 6.16. Detalle del registro obtenido. No contiene otro procesamiento más que la limitación de su ancho de banda a $128 \mathrm{~Hz}$.

\section{Conclusiones.}

Las soluciones pasivas presentan un bajo consumo y admiten altas $V_{\text {iDC.MAX }}$, pero producen elevadas tensiones de offset de salida $V_{\mathrm{oDC}}$ que obligan a incluir una segunda etapa de supresion de DC. Esto último atenta contra la respuesta transitoria, exigiendo un cuidadoso y comprometido diseño del A.B. (RAMOS, 2001), (SPINELLI, 2004).

La red de acoplamiento pasiva propuesta permite obtener muy altos CMRR conservando una implementación simple y totalmente pasiva.

Por otra parte, las estrategias activas permiten asegurar bajas tensiones de DC a la salida del A.B. y admiten la implementación de estrategias de recuperación rápida frente a perturbaciones que lleven a la saturación del A.B..

El principal inconveniente de la solución activa clásica es la limitación de la ganancia de la primer etapa a valores moderados. Para salvar esto, se propuso una implementación F-D del esquema activo que admite altas $V_{\text {i.DC.MAX }}$ aún con bajas tensiones de alimentación.

Respecto a la tensión eficaz de ruido, las soluciones analizadas, así también como las propuestas en esta tesis, presentan niveles de ruido similares.

A modo de resumen, a continuación se presenta una tabla comparativa entre las distintas alternativas para conseguir el acoplamiento en alterna. 


\begin{tabular}{|c|c|c|c|c|c|c|c|}
\hline & & $V_{\text {IDC.MAX }}$ & CMRR & $V_{O D . O U T}$ & $\mathrm{G}_{D D}$ & $Z_{c}$ & $e^{2}{ }_{i T}$ \\
\hline \multirow{2}{*}{ 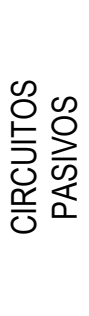 } & $\begin{array}{l}\text { Red T } \\
\text { (Clásica) }\end{array}$ & $\begin{array}{l}\text { No } \\
\text { limitado }\end{array}$ & Bajo & $\begin{array}{c}\text { Alto } \\
\left(\Delta \mathrm{V}_{\text {.oFFSET }} \cdot \mathrm{ADD}\right)\end{array}$ & Alta & baja & $\approx 2 \mathrm{e}^{2} \mathrm{AO}$ \\
\hline & $\begin{array}{c}\text { F-D } \\
\text { Balanceada } \\
\text { (Propuesta en } \\
\text { esta tesis) }\end{array}$ & $\begin{array}{c}\text { No } \\
\text { limitado }\end{array}$ & Alto & $\begin{array}{c}\text { Alto } \\
\Delta \mathrm{V}_{\text {.OFFSET }} \cdot \mathrm{GDD}^{\circ}\end{array}$ & Alta & $\begin{array}{l}\text { Alta } \\
\text { (CiN) }\end{array}$ & $\approx 2 \mathrm{e}^{2} \mathrm{AO}$ \\
\hline \multirow{2}{*}{ 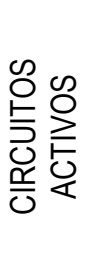 } & $\begin{array}{l}\text { Activo S-E } \\
\text { (Clásico) }\end{array}$ & $\begin{array}{c}\text { Bajo } \\
\left(V_{c C} / A_{D D}\right)\end{array}$ & Moderado & $\begin{array}{l}\text { Muy Bajo } \\
\left(\Delta \mathrm{V}_{\text {ofFSET }}\right)\end{array}$ & Moderada & $\begin{array}{l}\text { Alta } \\
\left(\mathrm{C}_{\mathrm{IN}}\right)\end{array}$ & $\approx 2 \mathrm{e}^{2} \mathrm{AO}$ \\
\hline & $\begin{array}{l}\text { Activo F-D } \\
\text { (Propuesta en } \\
\text { esta tesis) }\end{array}$ & $\begin{array}{c}\text { Moderado } \\
\left(2 V_{c c} / \beta\right)\end{array}$ & Alto & $\begin{array}{c}\text { Moderado } \\
\left(\Delta \mathrm{V}_{\text {.OFFSET }} \cdot \mathrm{G}_{\mathrm{DD}} / \beta\right)\end{array}$ & Alta & $\begin{array}{l}\text { Alta } \\
\text { (CIN) }\end{array}$ & $\approx 2 \mathrm{e}^{2} \mathrm{AO}$ \\
\hline
\end{tabular}

Tabla 1. Factores de Mérito de las distintas técnicas para Acoplamiento en Aterna analizadas.

Detalle de los símbolos utilizados en la Tabla 1.

$V_{\text {iDC.MAX }}:$ máxima tensión de entrada DC admisible.

CMRR : Rechazo de Modo Común.

$V_{\text {oDC.out }}:$ Tensión de DC a la salida del A.B.

$G_{\mathrm{DD}} \quad$ : Ganancia admisible en el Front-End.

$Z_{\mathrm{C}} \quad:$ impedancia de entrada de modo común

$V_{\text {OFFSET }}:$ tensión de offset de un A.O.

$\Delta V_{\text {OFFSET }}$ : diferencia entre las tensiones de offset entre dos A.O.

$\beta \quad:$ Parámetro de diseño del A.B. F-D de dos A.O. Usualmente $\beta \approx 10 \ll G_{\mathrm{DD}}$.

$C_{\mathrm{IN}} \quad:$ Capacidad de Entrada del A.O.

$e^{2}{ }_{\text {iT }} \quad$ : ruido RMS al cuadrado total referido a la entrada.

$e^{2}{ }_{\text {AO }} \quad$ : ruido RMS al cuadrado de un A.O.

$V_{\mathrm{CC}} \quad:$ Tensión de alimentación. 


\section{Capítulo 7: Mejora de las características de entrada de un A.B. mediante Realimentación de Modo Común.}

\section{Resumen.}

Es un hecho bien conocido que mediante el uso de realimentación negativa es posible mejorar ciertas características de un amplificador, como la impedancia de entrada, linealidad, rechazo a perturbaciones, etc. En este capítulo se presentan varias estrategias, basadas en la realimentación de la tensión de modo común, que permiten mejorar sustancialmente las propiedades de un A.B.

Un amplificador de instrumentación generalmente presenta muy elevadas impedancias de entrada de modo común $Z_{\mathrm{C}}$. En aplicaciones biomédicas, esta impedancia se ve comprometida por necesidad de disponer de un camino para las corrientes de polarización de entrada y por la capacidad de los cables blindados. Se presentan aquí, técnicas como bootstraping y circuitos manejadores de blindaje (shield-driver) que permiten minimizar estos efectos.

También se analiza en este capítulo, un circuito denominado DRL (Driven Right Leg) que permite reducir la tensión de modo común $V_{\mathrm{CM}}$ mediante una realimentación negativa de la misma. Esta reducción de $V_{\mathrm{CM}}$ equivale a incrementar el rechazo del A.B. a interferencias de modo común.

En la última sección, se aplica la realimentación de modo común para el control a lazo cerrado del punto de polarización de amplificadores alimentados con una Fuente Simple.

Todos estos circuitos operan realimentando $V_{\mathrm{CM}}$ y pueden presentar problemas de estabilidad. En este capitulo se analizan las condiciones de estabilidad para cada uno de los circuitos y también para el caso en que operen en forma conjunta. 


\section{Reducción de la tensión de modo común. El circuito DRL.}

Según se analizó en detalle en el CAPÍtulo 2, la tensión de modo común $V_{\mathrm{CM}}$ es producida principalmente por la tensión de red. A los efectos del análisis y del diseño de los circuitos reductores de modo común, resulta suficiente el modelo simplificado de la Fig.6.1. Entre otras cosas, este esquema considera una capacidad línea-masa $\left(C_{\text {SUP }}\right)$ despreciable.

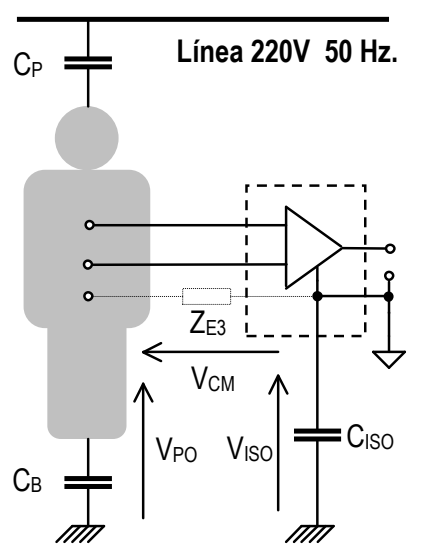

Figura 6.1. Modelo de EMI simplificado utilizado para el análisis del circuito reductor de la tensión de modo común.

Una forma de obtener tensiones de modo común reducidas es asegurando que la impedancia del tercer electrodo $Z_{\mathrm{E} 3}$ (paciente-masa) sea baja. Esto no es simple de conseguir, pues esta impedancia presenta valores poco predecibles de entre $1 \mathrm{k} \Omega$ y $1 \mathrm{M} \Omega$ (Rossel, 1988).

Como se comentó anteriormente, una forma de obtener bajas impedancias electrodopiel es "preparando" la zona donde se aplica el electrodo. Una técnica simple, aunque algo primitiva, consiste en eliminar la primer capa (de alta impedancia) de la epidermis ${ }^{1}$ mediante abrasión. Es un objetivo de la ingeniería biomédica eliminar este tipo de "incomodidades".

Una forma de asegurar una $V_{\mathrm{CM}}$ reducida, aún con altas impedancias electrodo piel, es utilizando una realimentación negativa de la tensión de modo común a través del electrodo de masa $Z_{\mathrm{E} 3}$. Esta forma de utilizar el tercer electrodo, incorporándolo a un circuito activo reductor de modo común, se conoce como "circuito excitador de pierna derecha", "Driven-Right-Leg-Circuit" o simplemente DRL. Este nombre algo extraño se debe al hecho que, en electrocardiografía, el electrodo de masa se coloca en la pierna derecha.

\footnotetext{
1 Se realizaron algunas pruebas que permitieron comprobar que efectuando una abrasión importante ( $\sin$ llegar a ver sangre) las impedancias de contacto toman valores menores a $200 \Omega$ !!!. En estas condiciones es muy simple medir y esta tesis no tendría mucho sentido.
} 
En la Fig.6.2 se muestra un esquema de este tipo de circuito. A partir del A.B. se obtiene $^{2}$ una muestra de la tensión de modo común de entrada $V_{\mathrm{CM}}$. Ésta es realimentada en forma negativa al tercer electrodo luego de ser comparada con el potencial de masa y amplificada por $A(\mathrm{~s})$. Este esquema incluye filtros pasivos $\mathrm{RC}$ en las entradas del A.B., los cuales quedan establecidos ${ }^{3}$ por la impedancia propia del electrodo y la capacidad $C_{\mathrm{F}}$ de los cables de conexión.

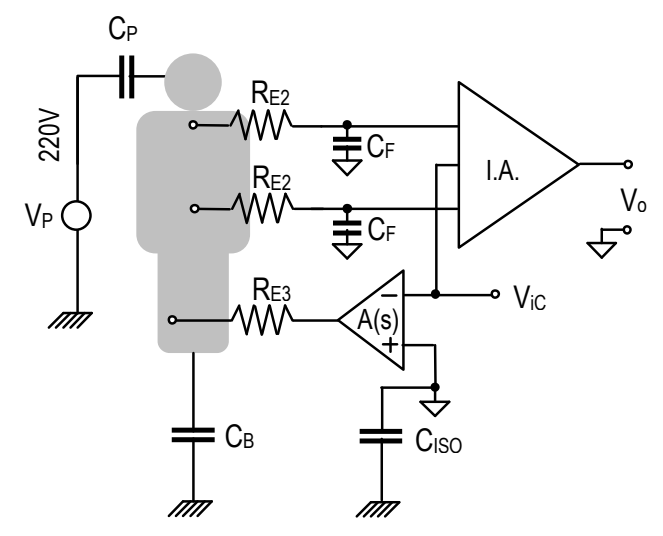

Figura 6.2. Esquema general de un A.B. con circuito DRL. El potencial indicado como $V_{i c}$ corresponde a la tensión de modo común presente en las entradas del amplificador.

\subsection{Estabilidad del Circuito DRL.}

A partir del esquema presentado en la Fig.6.2 se obtiene, para tensiones de modo común, el circuito de la Fig. 6.3. Para llegar a este esquema simplificado se consideró que la muestra de $V_{\text {ic }}$ provista por el I.A. coincide con la tensión de modo común presente en sus entradas ${ }^{4}$.

\footnotetext{
${ }^{2}$ Más adelante, en la Fig.6.4 se detallará como puede obtenerse una muestra de la tensión de modo común de entrada.

${ }^{3}$ En algunos casos estos filtros no son "parásitos" sino que se incluyen especialmente para atenuar componentes de alta frecuencia.

${ }^{4}$ Esto implica suponer que el amplificador de instrumentación tiene un producto ganancia ancho de banda GPB suficiente como para no incorporar singularidades dentro del rango de frecuencias de interés.
} 


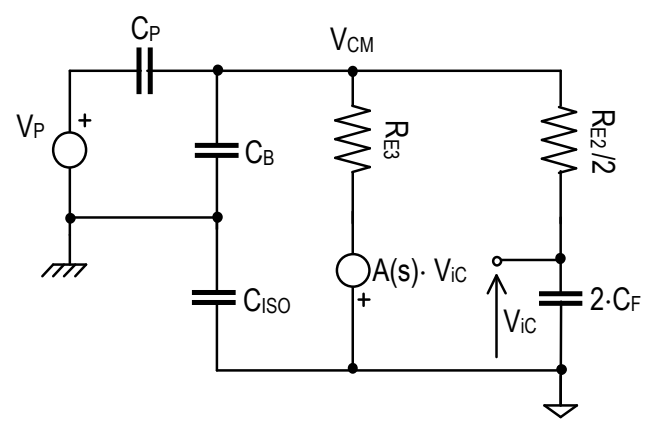

Figura 6.3. Circuito equivalente del esquema de la Fig. 6.2.

Resolviendo el circuito se obtiene que la relación entre la tensión de modo común $V_{\mathrm{CM}}$ y la tensión de línea $V_{\mathrm{P}}$ está dada por:

$$
\left.\frac{V_{\mathrm{CM}}}{V_{\mathrm{P}}}\right|_{\text {Clasico }}=K_{\mathrm{C}} \cdot s \frac{\frac{\left(1+s \cdot \tau_{0}\right) R_{\mathrm{E} 3}}{s^{2} \cdot \tau_{0} \tau_{1}+s\left(\tau_{0}+\tau_{1}+\tau_{2}\right)+1}}{1+\frac{A(s)}{s^{2} \cdot \tau_{0} \tau_{1}+s\left(\tau_{0}+\tau_{1}+\tau_{2}\right)+1}}
$$

siendo

$$
\begin{aligned}
& R_{0}=\frac{R_{\mathrm{E} 2}}{2} ; \quad C_{\mathrm{N}}=\frac{C_{\mathrm{ISO}} \cdot\left(C_{\mathrm{P}}+C_{\mathrm{B}}\right)}{C_{\mathrm{ISO}}+C_{\mathrm{P}}+C_{\mathrm{B}}} \quad ; \quad K_{C}=\frac{C_{P} \cdot C_{I S O}}{C_{I S O}+C_{P}+C_{B}} \\
& C_{0}=2 C_{\mathrm{F}} \quad ; \quad \tau_{0}=R_{0} C_{0} \quad ; \quad \tau_{1}=R_{\mathrm{E} 3} C_{\mathrm{N}} \quad ; \quad \tau_{2}=R_{\mathrm{E} 3} C_{0}
\end{aligned}
$$

La estabilidad del DRL puede analizarse a partir de su ganancia a lazo abierto $G H(\mathrm{~s})$, la cual puede obtenerse por inspección de (6.1):

$$
G H(s)=\frac{1}{s^{2} \cdot \tau_{0} \tau_{1}+s\left(\tau_{0}+\tau_{1}+\tau_{2}\right)+1} \cdot A(s)
$$

En el diseño de la compensación de este circuito debe tenerse en cuenta que muchos de los parámetros involucrados no son bien conocidos y además muy variables. Por este motivo los polos del sistema no están definidos con precisión.

Para asegurar la estabilidad del DRL (WINTER \& WEBSTER, 1983) proponen, con un criterio muy razonable dada la incertidumbre en los parámetros involucrados, una compensación por polo dominante considerando un caso desfavorable.

En (6.2) se observa que el primer factor, asociado a los elementos pasivos del circuito, presenta una ganancia aproximadamente unitaria para frecuencias por debajo de la correspondiente al primer polo. La compensación resulta así relativamente simple; basta con utilizar como amplificador de modo común $A(s)$ un integrador ${ }^{5}$ tal que su

\footnotetext{
${ }^{5}$ Dado que el integrador se implementará con un A.O. real con una ganancia de de lazo abierto finita $A_{0}$, el polo dominante no estará en el origen como sugiere (6.3) sino que su constante de
} 
ganancia decaiga a 0dB ("corte") antes del primer polo de (6.2). La transferencia de este amplificador será:

$$
A(s)=\frac{1}{R_{\mathrm{i}} C_{\mathrm{i}}}
$$

Un circuito algo más detallado del DRL, incorporando este integrador como amplificador de modo común, se muestra en la Fig.6.4. El resistor $R_{\mathrm{P}}$ limita la corriente máxima que puede circular por el paciente que de acuerdo a la norma (AAMI, 1998) no debe exceder los 10 $\mu \mathrm{A}$.

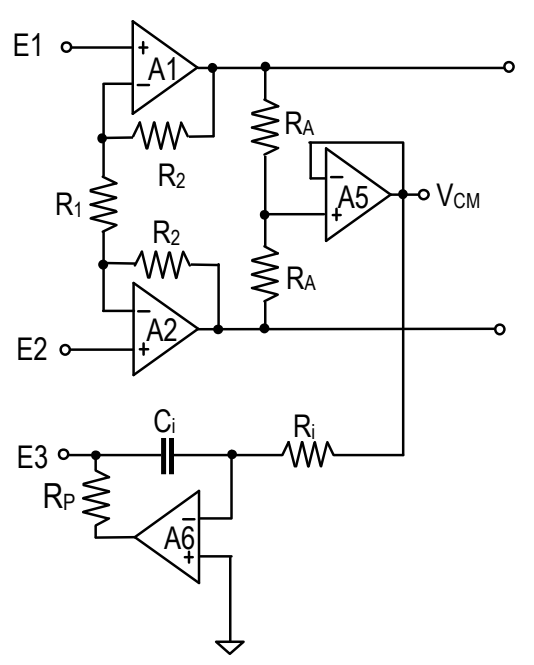

Figura 6.4. Circuito práctico para la implementación del DRL.

De aquí en más, el diseño del circuito DRL se reduce a dimensionar el integrador (es decir $R_{\mathrm{i}}$ y $C_{\mathrm{i}}$ ), de modo tal que su ganancia sea unitaria a la frecuencia del primer polo. Por ejemplo, considerando valores no muy optimistas ${ }^{6}$ : $C_{\mathrm{ISO}}=200 \mathrm{pF}, C_{\mathrm{P}}=2 \mathrm{pF}$, $C_{\mathrm{B}}=200 \mathrm{pF}, C_{\mathrm{F}}=200 \mathrm{pF}, R_{\mathrm{E} 1}=100 \mathrm{k} \Omega, R_{\mathrm{E} 3}=200 \mathrm{k} \Omega, R_{0}=10 \mathrm{k} \Omega, A_{0}=110 \mathrm{~dB}$, que determinan $R_{0}=50 \mathrm{~K}, C_{0}=400 \mathrm{pF}, C_{\mathrm{N}}=100 \mathrm{pF}, \tau_{0}=22 \mu \mathrm{S}, \tau_{1}=20 \mu \mathrm{S}, \tau_{2}=80 \mu \mathrm{S}$, resulta:

$$
\begin{aligned}
& G H(s)=\frac{A(s)}{\left(s^{2} \cdot 440 \cdot 10^{-12}+s \cdot 122 \cdot 10^{-6}+1\right)} \\
& G H(s)=\frac{A(s)}{(1+s \cdot 117 \mu \mathrm{s}) \cdot(1+s \cdot 3.7 \mu \mathrm{s})}
\end{aligned}
$$

tiempo asociada será $\tau_{\mathrm{AO}}=R_{\mathrm{i}} \cdot C_{\mathrm{i}} \cdot A_{0}$. De todos modos, para el análisis de estabilidad (frecuencias próximas al corte de $0 \mathrm{~dB}$ ) puede considerarse un integrador ideal.

${ }^{6}$ Se adoptaron valores elevados para las capacidades de acoplamiento, muy elevados para $C_{\mathrm{ISO}}$, una alta impedancia electrodo piel para los electrodos de señal y una muy elevada para el tercer electrodo de masa. 
El polo de menor frecuencia se ubica en $1.36 \mathrm{kHz}$ y el superior en $43 \mathrm{kHz}$. Para que la ganancia del integrador sea unitaria en $1.36 \mathrm{kHz}$ se debe verificar:

$$
\frac{1}{R_{i} C_{i} \cdot 2 \pi \cdot 1.36 \mathrm{kHz}}=1
$$

de donde resulta: $R_{\mathrm{i}} C_{\mathrm{i}}=117 \mu \mathrm{s}$. Para utilizar valores comerciales se puede adoptar:

$$
R_{\mathrm{i}} C_{\mathrm{i}}=10 \mathrm{k} \Omega \cdot 10 \mathrm{nF}=100 \mu \mathrm{s}
$$

y la ganancia a lazo abierto del sistema compensado queda:

$$
G H(\mathrm{~s})=\frac{1}{s \cdot 100 \mu s \cdot(1+s \cdot 117 \mu \mathrm{s}) \cdot(1+s \cdot 3.7 \mu \mathrm{s})}
$$

En la Fig.6.5 se muestra la ganancia de lazo abierto correspondiente al sistema compensado, donde se observa que la ganancia a $50 \mathrm{~Hz}$ es de alrededor de $30 \mathrm{~dB}^{7}$ y su margen de fase de unos 45 grados.

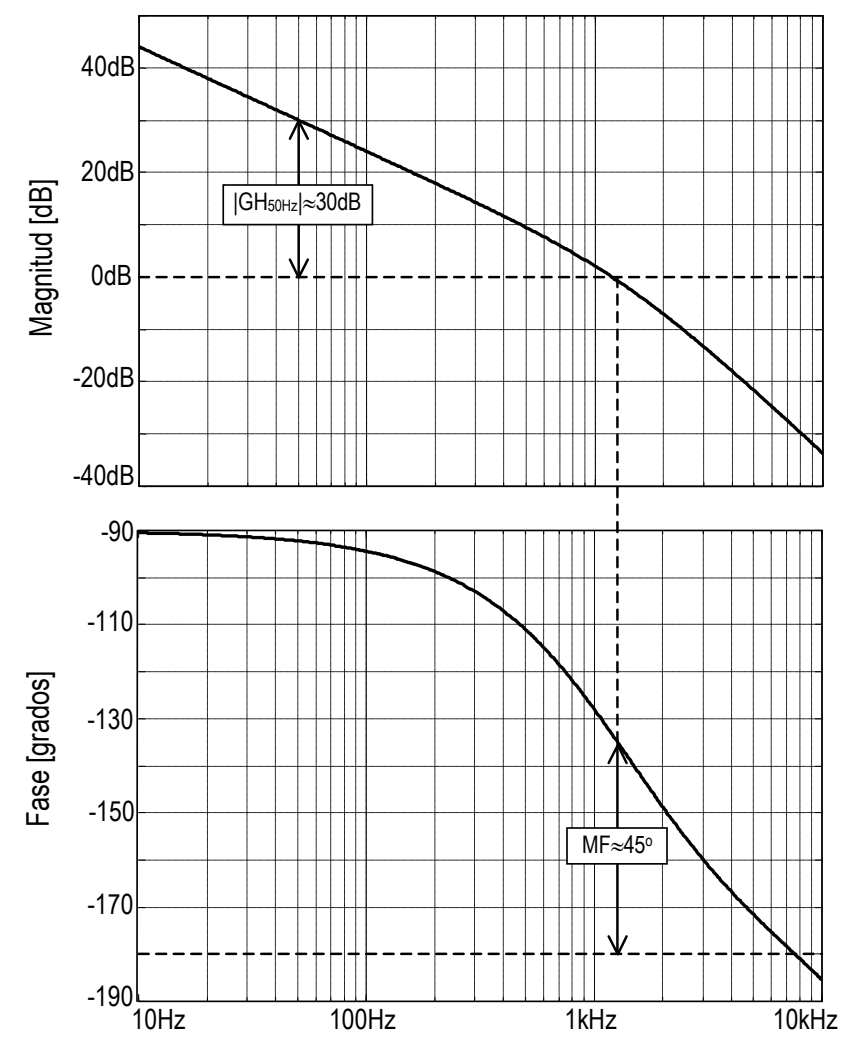

Figura 6.5. Ganancia de Lazo Abierto del sistema compensado. El mismo presenta un Margen de Fase de unos $45^{\circ}$.

\footnotetext{
${ }^{7}$ Este valor está dado por la ganancia del integrador para esta frecuencia.
} 


\subsection{Rechazo de la Tensión de línea.}

Reemplazando la expresión de $A(\mathrm{~s})$ dada por (6.3) en (6.1) se obtiene:

$$
\left.\frac{V_{\mathrm{CM}}}{V_{\mathrm{P}}}\right|_{\text {Clásico }}=K_{\mathrm{C}} \cdot R_{\mathrm{E} 3} \cdot \frac{s^{2} \cdot\left(1+s \cdot \tau_{0}\right) \cdot R_{\mathrm{i}} C_{\mathrm{i}}}{\left(1+s \cdot\left(\tau_{0}+\tau_{1}+\tau_{2}\right)+s^{2} \cdot \tau_{0} \cdot \tau_{1}\right) \cdot s \cdot R_{\mathrm{i}} C_{\mathrm{i}}+1},
$$

que con los valores anteriormente adoptados se transforma en:

$$
\left.\frac{V_{\mathrm{CM}}}{V_{\mathrm{P}}}\right|_{\text {Clásico }}=1 \mathrm{pF} \cdot 200 \mathrm{k} \Omega \cdot \frac{s^{2}(1+s \cdot 22 \mu \mathrm{s})(1+s \cdot 31.6 \mathrm{~s}) \cdot 100 \mu \mathrm{s}}{s\left(1+s \cdot 122 \mu \mathrm{s}+s^{2} \cdot 440 \mu \mathrm{s}^{2}\right) \cdot 100 \mu \mathrm{s}+1} .
$$

En la Fig.6.6 se muestra la respuesta en frecuencia correspondiente a esta función de transferencia (en línea llena) y la misma función para el caso en que el paciente sea conectado a masa mediante una resistencia de $200 \mathrm{k} \Omega$ sin utilizar el DRL. Esto último corresponde a la expresión (6.9) considerando $R_{\mathrm{i}} C_{\mathrm{i}}=\infty$ (ganancia nula).

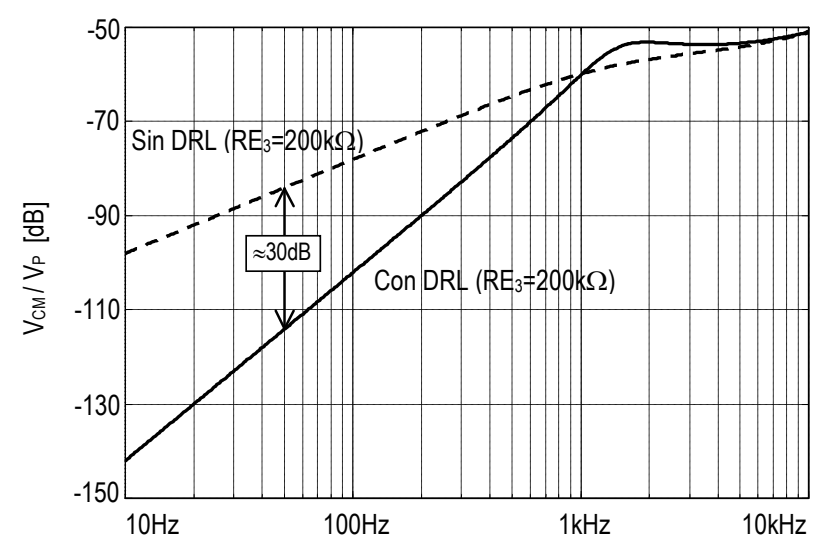

Figura 6.6. Rechazo de la Tensión de Línea. La curva en línea llena corresponde al caso de utilizar el circuito DRL. En línea punteada se indica el rechazo correspondiente a la conexión directa del paciente a masa mediante una impedancia $R_{E 3}=200 \mathrm{k} \Omega$.

En esta figura se observa, que a la frecuencia de la tensión de red $(50 \mathrm{~Hz}$.), la tensión de modo común está unos $120 \mathrm{~dB}$ por debajo de la tensión de línea. Considerando una red de $V_{\mathrm{P}}=220 \mathrm{~V}$ resulta una tensión $V_{\mathrm{CM}}$ de alrededor de $220 \mu \mathrm{V}$ que no produciría mayores inconvenientes ${ }^{8}$. En esta figura también se observa que el nivel de $V_{\mathrm{CM}}$ utilizando el DRL es unos $30 \mathrm{~dB}$ menor que el correspondiente a la conexión directa a través del tercer electrodo.

\footnotetext{
${ }^{8}$ Este rechazo generalmente es suficiente para que la interferencia introducida por $V_{\mathrm{CM}}$ no sea apreciable. De todos modos, la compensación diseñada es bastante conservadora y si fuera necesario podría asignarse una ganancia más importante.
} 
Como muestra la Fig.6.5, para frecuencias elevadas, la ganancia de lazo abierto es reducida, conduciendo a un bajo rechazo de EMI en estas frecuencias. En general, esto no presenta un inconveniente grave, pero en presencia de interferencia de alta frecuencia, por ejemplo debida a tubos fluorescentes (WINTER \& WEBSTER, 1983), puede dar lugar a importantes niveles de interferencia.

Los tubos fluorescentes utilizados en iluminación generan EMI a la frecuencia de línea y también componentes de alta frecuencia de alrededor de $1 \mathrm{kHz}$. (WINTER, 1983), (SILVA, 1994). Si bien la energía de este tipo de EMI en alta frecuencia no es importante, su valor pico puede ser comparable al de la componente de $50 \mathrm{~Hz}$ (ver APÉNDICE 11) y resulta deseable contar con un buen rechazo de modo común para frecuencias altas. Una solución para estos casos, es utilizar un amplificador de modo común de transconductancia con un ancho de banda extendido. (SPINELLI, 1999).

\section{Circuito Manejador de Pantalla (S-D: Shield-Driver)}

Como se mostró en el CAPÍTULO 2, los cables blindados son necesarios para eliminar la interferencia producida por acoplamiento a los cables de conexión. Este tipo de cable presenta una capacidad importante ${ }^{9}$ entre el conductor central y su malla, lo cual degrada sensiblemente la impedancia de entrada de modo común. Para evitar esto, puede utilizarse un "circuito manejador de pantalla" más conocido como "ShieldDriver" (S-D).

Este tipo de circuito, que se muestra en la Fig.6. $7^{10}$, intenta mantener la malla del cable al potencial de su conductor interno. De esta manera, no circulará corriente alguna a través de la capacidad distribuida del cable $C_{\mathrm{SH}}$ y se minimizarán sus efectos.

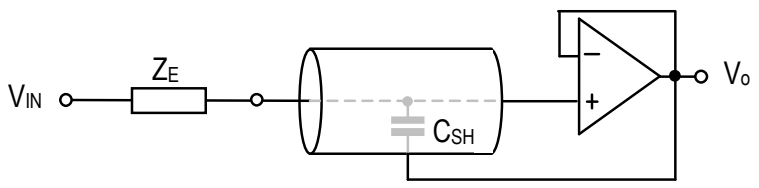

Figura 6.7. Circuito Shield-Driver.

Considerando la capacidad distribuida del cable como una capacidad concentrada $C_{\mathrm{SH}}$, incorporando la capacidad de entrada propia del A.O. $\left(C_{\mathrm{IN}}\right)$ y representando el buffer por su ganancia $G(s)$, resulta el circuito equivalente de la Fig.6.8.

\footnotetext{
${ }^{9}$ Dependiendo del diámetro del cable, su capacidad de hasta varias centenas de $\mathrm{pF}$ por metro.

${ }^{10}$ En realidad se trata de un circuito equivalente para tensiones de modo común.
} 


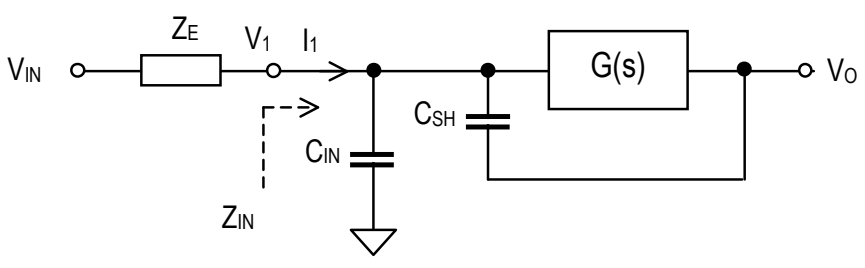

Figura 6.8. Circuito equivalente del esquema de la Fig. 6.7

Se advierte en esta figura, que el circuito contiene una realimentación positiva a través de la capacidad $C_{\mathrm{SH}}$ lo cual sugiere la posibilidad de que existan problemas de estabilidad. Para el análisis de este circuito, en primer lugar se calculará la impedancia de entrada $Z_{\mathrm{IN}}$ vista desde el cable. Posteriormente, se incluirá la impedancia de electrodo $Z_{\mathrm{E}}$ para finalmente determinar la función de transferencia entre la tensión de salida $V_{\mathrm{O}}$ y la de entrada $V_{\mathrm{IN}}$.

\subsection{Impedancia de Entrada.}

La impedancia de entrada del amplificador con el shield-driver, definida como $V_{1} / I_{1}$ (ver Fig.6.8) está dada por:

$$
Z_{\mathrm{IN}}=\frac{V_{1}}{I_{1}}=\frac{1}{s\left(C_{\mathrm{IN}}+C_{\mathrm{SH}}(1-G(s))\right)}
$$

En esta ecuación se observa que para frecuencias bajas, donde $G(s)$ es aproximadamente unitaria, la impedancia de entrada está determinada por la capacidad de entrada propia del A.O. y es independiente de la capacidad del blindaje $C_{\mathrm{SH}}$ : $\operatorname{los}$ efectos de esta última capacidad son "neutralizados"

\subsection{Función de Transferencia.}

Considerando una impedancia de electrodo resistiva $R_{\mathrm{E}}$, la función transferencia del circuito shield-driver queda dada por:

$$
T(s)=\frac{V_{\mathrm{o}}(s)}{V_{\mathrm{IN}}(s)}=\frac{Z_{\mathrm{IN}}(s)}{R_{\mathrm{E}}+Z_{\mathrm{IN}}(s)} .
$$

Reemplazando (6.11) en (6.12) resulta:

$$
T(s)=\frac{1}{s\left(\tau_{i}+\tau_{G}(1-G(s))\right)+1} \quad ; \quad \operatorname{con} \tau_{\mathrm{G}}=R_{\mathrm{E}} C_{\mathrm{SH}} ; \tau_{\mathrm{i}}=R_{\mathrm{E}} C_{\mathrm{IN}}
$$

Caso 1. G(s) de primer orden.

La función de transferencia hallada en el punto previo depende de la ganancia $G(s)$ del buffer. Si se considera que esta ganancia verifica un modelo de primer orden (METTING VAN RIJN, 1990), y un A.O. con un producto ganancia-ancho de banda GPB, esta ganancia $G(s)$ tendrá la forma:

$$
G(s)=\frac{1}{1+s \cdot \tau_{\mathrm{A}}} \quad ; \quad \tau_{\mathrm{A}}=1 /(2 \pi \mathrm{GPB}) \quad .
$$

Remplazando (6.14) en (6.13) resulta: 


$$
T(s)=\frac{1+s \cdot \tau_{\mathrm{A}}}{s^{2} \cdot \tau_{\mathrm{A}}\left(\tau_{\mathrm{G}}+\tau_{\mathrm{i}}\right)+\left(\tau_{\mathrm{i}}+\tau_{\mathrm{A}}\right) s+1} .
$$

Como $C_{\mathrm{IN}} \ll C_{\mathrm{SH}}$, es decir $\tau_{\mathrm{i}} \ll \tau_{\mathrm{G}}$, la ecuación (6.15) puede aproximarse aceptablemente por:

$$
T(s)=\frac{1+s \cdot \tau_{\mathrm{A}}}{s^{2} \cdot \tau_{\mathrm{A}} \tau_{\mathrm{G}}+\left(\tau_{\mathrm{i}}+\tau_{\mathrm{A}}\right) s+1}
$$

Dado que el polinomio característico es de segundo orden con coeficientes positivos, el sistema es incondicionalmente estable. Es decir, que para una $G(s)$ dada por (6.14), el circuito es estable para cualquier valor de sus componentes $\left(C_{\mathrm{SH}}, C_{\mathrm{IN}}, R_{\mathrm{E}}\right)$.

Si bien en estas condiciones el circuito no es inestable, su respuesta transitoria puede ser poco amortiguada. La frecuencia de resonancia $f_{\mathrm{n}}$, y el factor de amortiguamiento están dados por:

$$
f_{\mathrm{n}}=\frac{1}{2 \pi \sqrt{\tau_{\mathrm{A}} \tau_{\mathrm{G}}}} ; \quad \xi=\sqrt{\frac{\tau_{\mathrm{A}}}{4 \tau_{\mathrm{G}}}}+\tau_{\mathrm{i}} \sqrt{\frac{1}{4 \tau_{\mathrm{A}} \tau_{\mathrm{G}}}}
$$

Como $\tau_{\mathrm{G}} \gg \tau_{\mathrm{A}}$, a partir de (6.16) se observa que el ancho de banda del S-D es sensiblemente menor que el GPB del A.O. Se observa además, que la capacidad de entrada $C_{\mathrm{IN}}$ incrementa el amortiguamiento $\left(C_{\mathrm{IN}}\right.$ incrementa $\left.\tau_{i}\right)$.

Por ejemplo, considerando un cable blindado de unos $2 \mathrm{~m}$ de longitud con una capacidad de $100 \mathrm{pF} / \mathrm{m}\left(C_{\mathrm{SH}}=200 \mathrm{pF}\right), R_{\mathrm{E}}=100 \mathrm{k} \Omega$ y un A.O. con un GBP de $1 \mathrm{MHz}$ y $C_{\mathrm{IN}}=3 \mathrm{pF}$ resultan:

$$
f_{n} \cong 90 \mathrm{kHz} \quad ; \quad \xi=0.1343 \quad .
$$

Caso 2. $G(s)$ de segundo orden.

Para conseguir una $G(s)$ de primer orden como la indicada en (6.14), es necesario utilizar un A.O especialmente diseñado para disponer de un buen margen de fase (MF). Su margen de fase debería ser de 90 grados.

Si se utiliza un A.O. de propósitos generales internamente compensado, el MF difícilmente superará los 45 grados. En este caso, $G(\mathrm{~s})$ presentará al menos dos polos y el circuito S-D sí puede presentar problemas de estabilidad.

Considerando que el buffer presenta una $G(\mathrm{~s})$ de segundo orden dada por:

$$
G(s)=\frac{\omega_{\mathrm{nA}}^{2}}{s^{2}+2 \xi_{\mathrm{A}} \omega_{\mathrm{nA}} s+\omega_{\mathrm{nA}}^{2}}
$$

y reemplazando esta $G(s)$ en (6.13) resulta:

$$
T(s)=\frac{s^{2}+2 \xi \omega_{\mathrm{nA}} s+\omega_{\mathrm{nA}}^{2}}{s^{3}\left(\tau_{i}+\tau_{\mathrm{G}}\right)+s^{2}\left[2 \xi_{\mathrm{A}} \omega_{\mathrm{nA}}\left(\tau_{\mathrm{i}}+\tau_{\mathrm{G}}\right)+1\right]+s\left(\omega_{\mathrm{nA}}^{2} \tau_{i}+2 \xi \omega_{\mathrm{nA}}\right)+\omega_{\mathrm{nA}}^{2}}
$$


Teniendo en cuenta que $\tau_{\mathrm{i}} \ll \tau_{\mathrm{G}},(6.20)$ puede aproximarse por:

$$
T(s) \cong \frac{s^{2}+2 \xi_{\mathrm{A}} \omega_{\mathrm{nA}} s+\omega_{\mathrm{nA}}^{2}}{s^{3} \tau_{\mathrm{G}}+s^{2}\left(2 \xi_{\mathrm{A}} \omega_{\mathrm{nA}} \tau_{\mathrm{G}}+1\right)+s\left(\omega_{\mathrm{nA}}^{2} \tau_{\mathrm{i}}+2 \xi_{\mathrm{A}} \omega_{\mathrm{nA}}\right)+\omega_{n \mathrm{~A}}^{2}} .
$$

y su polinomio característico es:

$$
s^{3} \tau_{\mathrm{G}}+s^{2}\left(2 \xi_{\mathrm{A}} \omega_{\mathrm{nA}} \tau_{\mathrm{G}}+1\right)+s\left(\omega_{\mathrm{nA}}^{2} \tau_{\mathrm{i}}+2 \xi_{\mathrm{A}} \omega_{\mathrm{nA}}\right)+\omega_{\mathrm{nA}}^{2}
$$

Este nuevo sistema, bajo ciertas condiciones, puede volverse inestable. Para determinar su estabilidad puede utilizarse el criterio de Routh. El arreglo correspondiente resulta:

$$
\begin{array}{ccc}
s^{3} & \tau_{\mathrm{G}} & \omega_{\mathrm{nA}}^{2} \tau_{\mathrm{i}}+2 \xi_{\mathrm{A}} \omega_{\mathrm{nA}} \\
s^{2} & 2 \xi_{\mathrm{A}} \omega_{\mathrm{nA}} \tau_{\mathrm{G}}+1 & \omega_{\mathrm{nA}}^{2} \\
s^{1} & {\left[\left(2 \xi_{\mathrm{A}} \omega_{\mathrm{nA}} \tau_{G}+1\right)\left(\omega_{\mathrm{nA}}^{2} \tau_{i}+2 \xi_{\mathrm{A}} \omega_{\mathrm{nA}}\right)-\tau_{\mathrm{G}} \omega_{\mathrm{nA}}^{2}\right] /\left(2 \xi_{\mathrm{A}} \omega_{\mathrm{nA}} \tau_{\mathrm{G}}+1\right)} & \\
s^{0} & \omega_{\mathrm{nA}}^{2} &
\end{array}
$$

El número de polos en el semiplano derecho está dado por los cambios de signo que se produzcan en la primer columna. Para que no existan cambios de signo, es decir para que el sistema sea estable, se debe verificar que:

$$
\left(2 \xi_{\mathrm{A}} \omega_{\mathrm{nA}} \tau_{\mathrm{G}}+1\right)\left(\omega_{\mathrm{nA}}^{2} \tau_{\mathrm{i}}+2 \xi_{\mathrm{A}} \omega_{\mathrm{nA}}\right)-\tau_{\mathrm{G}} \omega_{\mathrm{nA}}^{2}>0
$$

Reemplazando las expresiones correspondientes a $\tau_{\mathrm{i}}, \tau_{\mathrm{G}}$ y agrupando se obtiene:

$$
4 \xi_{\mathrm{A}}^{2}+2 \xi_{\mathrm{A}}\left(\omega_{\mathrm{nA}} R_{\mathrm{E}} C_{\mathrm{IN}}+\frac{1}{\omega_{\mathrm{nA}} R_{\mathrm{E}} C_{\mathrm{SH}}}\right)>1-C_{\mathrm{IN}} / C_{\mathrm{SH}}
$$

Considerando que $C_{\mathrm{IN}} / C_{\mathrm{SH}} \ll 1$, y definiendo una función $f\left(R_{\mathrm{E}}\right)$ como:

$$
f\left(R_{\mathrm{E}}\right)=4 \xi_{\mathrm{A}}^{2}+2 \xi_{\mathrm{A}}\left(\omega_{\mathrm{nA}} R_{\mathrm{E}} C_{\mathrm{IN}}+\frac{1}{\omega_{\mathrm{nA}} R_{\mathrm{E}} C_{\mathrm{SH}}}\right) \quad .
$$

La condición de estabilidad es:

$$
f\left(R_{\mathrm{E}}\right)>1 \quad,
$$

donde se observa claramente que la estabilidad del circuito depende de la impedancia $R_{\mathrm{E}}$ del generador. 
Una posible gráfica de $f\left(R_{\mathrm{E}}\right)$ se muestra en la Fig.6.10. En este caso, el circuito es inestable para un rango intermedio de valores de $R_{\mathrm{E}}$ mientras que es estable tanto para altos como para bajos valores de $R_{\mathrm{E}}$.

La peor condición, es decir el menor valor de $f\left(R_{\mathrm{E}}\right)$, se produce para

$$
R_{\mathrm{E} . \mathrm{C}}=\frac{1}{\omega_{\mathrm{nA}} \sqrt{C_{\mathrm{IN}} C_{\mathrm{SH}}}}
$$

y está dado por:

$$
f_{\mathrm{MIN}}=4 \xi_{\mathrm{A}}^{2}+4 \xi_{\mathrm{A}} \sqrt{C_{\mathrm{IN}} / C_{\mathrm{SH}}}
$$

El circuito será incondicionalmente estable si el mínimo valor de $f\left(R_{\mathrm{E}}\right)$ es mayor que la unidad, es decir:

$$
4 \xi_{\mathrm{A}}^{2}+4 \xi_{\mathrm{A}} \sqrt{C_{\mathrm{IN}} / C_{\mathrm{SH}}}>1
$$

Una buena opción para asegurar la estabilidad del S-D, independientemente de $C_{\mathrm{IN}} \mathrm{y}$ $C_{\mathrm{SH}}$ (los cuales pueden ser muy variables), es seleccionar un A.O tal que $4 \xi_{\mathrm{A}}^{2}$ sea mayor que uno, es decir con un $\xi_{\mathrm{A}}$ de al menos 0.5 .

En caso de ser necesario, otra forma de estabilizar el circuito es incluir un atenuador por un factor $\alpha$ a la salida del buffer (METTING VAN RIJN, 1990), reduciendo de esta forma su ganancia ligeramente por debajo de la unidad. Esta alternativa no es del todo recomendable pues degrada la reducción de $C_{\mathrm{SH}}$, subsistiendo una fracción remanente de $C_{\mathrm{SH}}$ dada por $C_{\mathrm{SH}}(1-\alpha)$.

\section{Observación.}

Para asegurar la estabilidad del circuito "shield-driver" es importante utilizar un A.O. especialmente diseñado para operar con ganancia unitaria (Buffer).

\subsection{Resultados Experimentales.}

Con el fin de validar las expresiones obtenidas, se efectuó un ensayo para observar la respuesta transitoria del circuito al aplicarle un escalón de tensión en su entrada.

En esta prueba se utilizó un cable coaxil con una capacidad total de $C_{\mathrm{SH}}=200 \mathrm{pF}$, una resistencia de generador $R_{\mathrm{E}}=100 \mathrm{k} \Omega$, y un A.O. LM358 trabajando con realimentación unitaria. En estas condiciones, la ganancia $G(s)$ del buffer depende, entre otras cosas, de la capacidad de carga aplicada a la salida del A.O. Para el dispositivo utilizado se encontró experimentalmente que su $G(s)$ corresponde aproximadamente a una función de transferencia según (6.19) con $\omega_{\mathrm{nA}} \approx 2 \pi \cdot 850 \mathrm{kHz}$ y $\xi_{\mathrm{A}} \approx 0.35$. 
En la Fig.6.9 se muestran la repuesta al escalón ${ }^{11}$ del circuito S-D correspondiente a la función de transferencia (6.21) y la obtenida en forma experimental, donde se observa un muy buen ajuste entre el modelo y los datos experimentales.

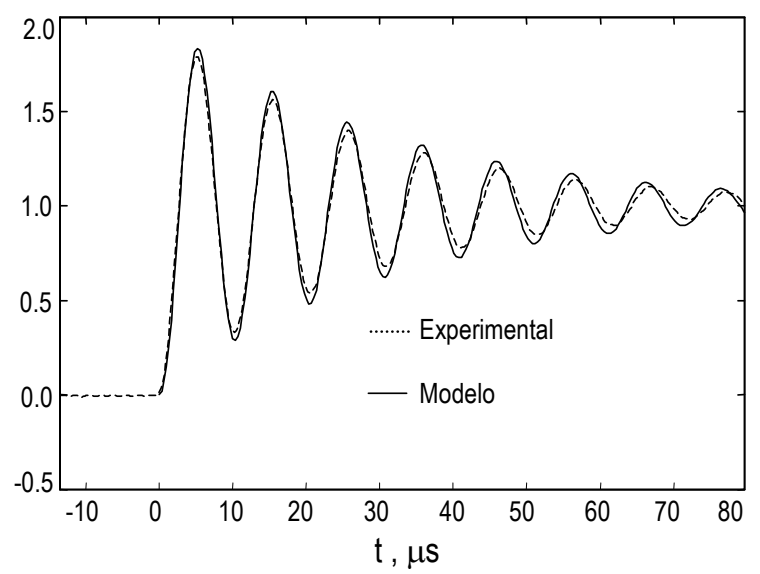

Figura 6.9.Respuesta al escalón del circuito S-D. En línea llena se indica la respuesta correspondiente a la expresión (6.20) y en línea punteada la obtenida en forma experimental.

En cuanto a la estabilidad del circuito construido, en la Fig.6.10 se muestra la gráfica correspondiente a la función $f\left(R_{\mathrm{E}}\right)$ definida anteriormente. Se observa que, para valores de $R_{\mathrm{E}}$ entre $1 \mathrm{k} \Omega$ y $40 \mathrm{k} \Omega$ el circuito es inestable $\left(f\left(R_{\mathrm{E}}\right)<1\right)$, mientras que fuera de este rango no presenta problemas de estabilidad. Esto fue comprobado experimentalmente: con $R_{\mathrm{E}}=50 \Omega$ y $R_{\mathrm{E}}=100 \mathrm{k} \Omega$ el circuito resultó estable, mientras que utilizando $R_{\mathrm{E}}=10 \mathrm{k} \Omega$ se tornó inestable.

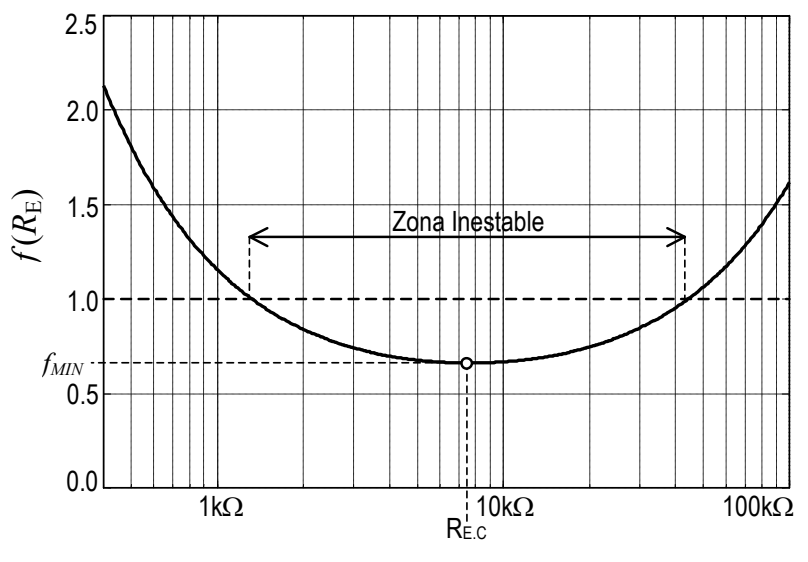

$\operatorname{RE}, \Omega$

Figura 6.10. Gráfica correspondiente a la función $f\left(R_{\mathrm{E}}\right)$ que define la estabilidad del circuito S-D. El mismo es inestable si $f\left(R_{\mathrm{E}}\right)$ adopta valores menores que la unidad.

\footnotetext{
${ }^{11} \mathrm{Si}$ bien en la Fig. 6.9 se indica la respuesta a un escalón unitario, la amplitud real del escalón aplicado fue de $50 \mathrm{mV}$ a fin de asegurar la operación del A.O. en zona lineal.
} 


\section{Estabilidad del DRL junto con el S-D.}

En caso de utilizar un circuito Shield-Driver (S-D), el análisis de estabilidad, así también como el diseño del DRL, difieren del presentado en la sección 2.1. Como se verá a continuación, en este caso las condiciones de operación son bastantes más benignas. Un modelo simplificado para el caso de utilizar simultáneamente los circuitos S-D y DRL se presenta en la Fig.6.11.

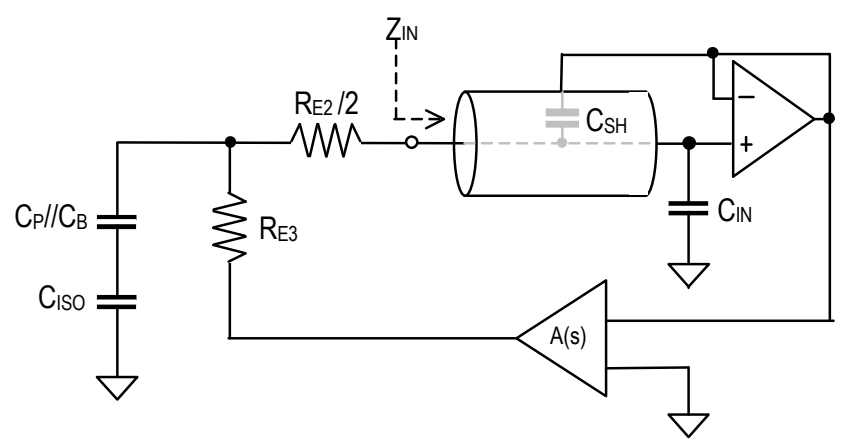

Figura 6.11. Circuitos S-D y DRL operando en conjunto.

Si se considera que el S-D es estable y que neutraliza efectivamente la capacidad $C_{\mathrm{SH}}$, la impedancia de entrada $Z_{\text {IN }}$ será muy elevada y el modelo de la Fig.6.11 puede reducirse al que se muestra en la Fig.6.12. Se observa aquí la importante contribución del S-D a la estabilidad de modo común: si bien agrega polos de alta frecuencia, elimina ${ }^{12}$ el polo impuesto por la capacidad propia del cable blindado.

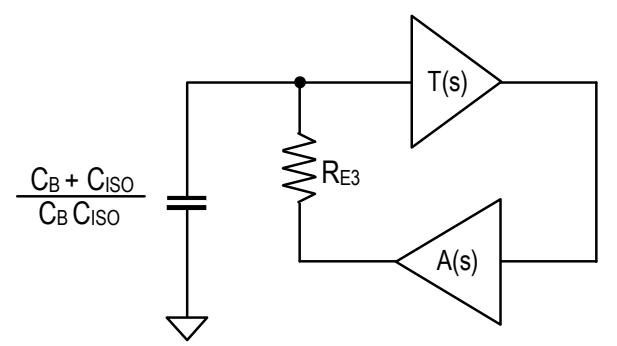

Figura 6.12. Circuito equivalente correspondiente al esquema de la Fig.6.11.

El nuevo modelo resulta verdaderamente simple. Su ganancia a lazo abierto $G H(s)$ está dada por:

$$
G H(s)=T(s) A(s) \frac{1}{1+s \tau_{\mathrm{SD}}} \quad ; \quad \tau_{\mathrm{SD}}=R_{\mathrm{E} 3} \frac{C_{\mathrm{B}} \cdot C_{\mathrm{ISO}}}{C_{\mathrm{B}}+C_{\mathrm{ISO}}}
$$

Siendo $T(s)$ la función transferencia del S-D y $A(\mathrm{~s})$ la correspondiente al DRL.

\footnotetext{
${ }^{12}$ No lo "elimina" pero lo traslada a frecuencias suficientemente altas como para no afectar al estabilidad del circuito.
} 


\subsection{Diseño del DRL con S-D.}

El nuevo sistema presenta un polo en $1 / \tau_{\mathrm{SD}}$ y polos de alta frecuencia aportados por el S-D. Utilizando amplificadores operacionales con GPB razonables $(\approx 1 \mathrm{MHZ})$ estos polos se producirán para frecuencias, del orden de $100 \mathrm{kHz}$.

Una forma simple de compensar el sistema es utilizando, al igual que en la sección 2.1, un integrador como implementación de la transferencia $A(\mathrm{~s})$. Su constante de tiempo debe dimensionarse para que el $A(s)$ presente ganancia unitaria en $1 / \tau_{\mathrm{SD}}$, asegurando de este modo un margen de fase de 45 grados. Es decir que la constante de tiempo del integrador debe ser igual o mayor que $\tau_{\mathrm{SD}}$. Considerando $C_{\mathrm{ISO}}=200 \mathrm{pF}$, $C_{\mathrm{B}}=200 \mathrm{pF}$ y $R_{\mathrm{E} 3}=100 \mathrm{k} \Omega$, de acuerdo a (6.31), la constante de tiempo $R_{\mathrm{i}} \mathrm{C}_{\mathrm{i}}$ resulta de $10 \mu \mathrm{s}$.

En la Fig.6.13 se muestra la ganancia de lazo abierto del sistema compensado. Se observa que el S-D desplaza el polo impuesto por la capacidad del cable blindado a frecuencias superiores, permitiendo otorgar mayor ganancia al sistema. En este caso, a la frecuencia de red $(50 \mathrm{~Hz})$ el DRL tendrá una ganancia a lazo abierto de alrededor de $50 \mathrm{~dB}$.
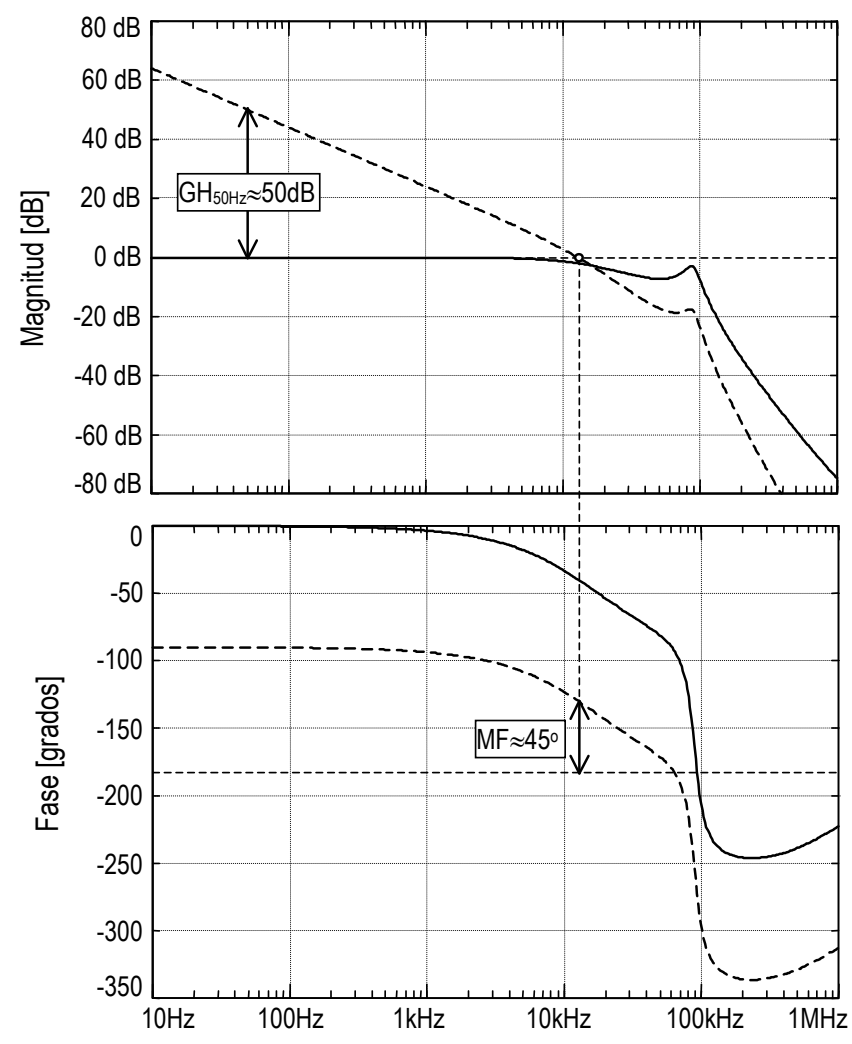

Figura 6.13. Ganancia de lazo abierto del sistema DRL+S-D. En línea llena: sistema sin compensar. En línea punteada: sistema compensado utilizando un integrador en el DRL. 


\section{Control de $Z_{c}$ mediante realimentación de modo común.}

Es un hecho conocido que por medio de una adecuada realimentación es posible modificar la impedancia de entrada de un amplificador.

En esta tesis se propone una estrategia, basada en una realimentación de la tensión de modo común, que permite modificar la impedancia de entrada para este modo $\left(Z_{\mathrm{C}}\right) \sin$ afectar la correspondiente a tensiones de modo diferencial $Z_{\mathrm{D}}$. Esto es particularmente útil en un A.B. porque se requiere una muy alta $Z_{\mathrm{C}}$, mientras que los requerimientos en cuanto a $Z_{\mathrm{D}}$ no son muy estrictos. Una $Z_{\mathrm{D}}$ de $10 \mathrm{M} \Omega$ cumple holgadamente con la norma AAMI. (AAMI, 1998)

La topología propuesta, introducida en el CAPÍTULO 5, se muestra en la Fig.6.14. Se trata de dos resistores $R_{\mathrm{P}}$ que definen la impedancia de entrada para señales de modo diferencial y un generador controlado de modo común que implementa la realimentación de modo común hacia la entrada. Estos elementos proveen un camino de DC a las corrientes de polarización del A.O.

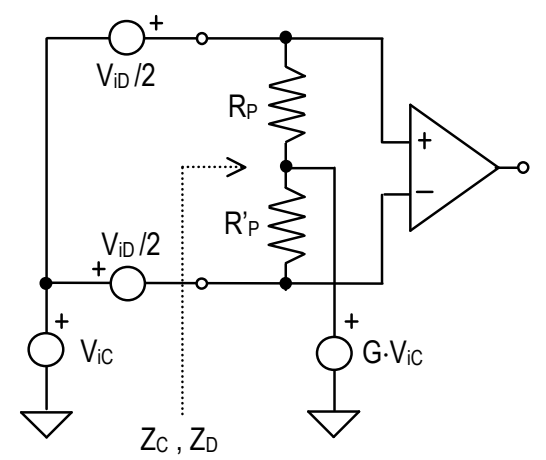

Figura 6.14. Topología propuesta para controlar Zc mediante una realimentación de Modo Común.

El circuito equivalente para tensiones de modo común es:

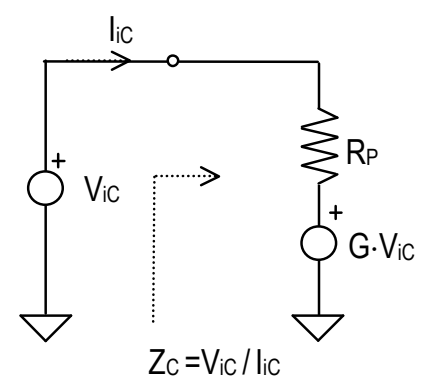

Figura 6.15. Circuito equivalente correspondiente al esquema de la Fig. 6.14.

A partir de este circuito puede obtenerse $Z_{\mathrm{C}}$, la cual está definida por:

$$
Z_{\mathrm{C}}=\frac{V_{\mathrm{iC}}}{I_{\mathrm{iC}}}
$$


resultando:

$$
Z_{\mathrm{C}}=\frac{R_{\mathrm{P}}}{1-G}
$$

Se observa que adoptando $G=1$ se obtiene $Z_{\mathrm{C}}=\infty$ y que, utilizando una ganancia negativa y muy elevada, es posible conseguir muy bajas $Z_{\mathrm{C}}$, lo cual puede resultar muy útil para sistemas de 2 electrodos ver (CAPÍtUlO 3). En ambos casos la impedancia de entrada de modo diferencial es igual a $2 R_{\mathrm{P}}$.

\subsection{Alta $Z_{c}$ : Amplificador con Bootstraping de Modo Común.}

Si se utiliza una ganancia $G$ unitaria, al aplicar una tensión de entrada de modo común, ambos bornes de los resistores $R_{\mathrm{P}}$ se encontrarán al potencial $V_{\mathrm{iC}}$. Sobre estos resistores no circularán corrientes y la impedancia $Z_{\mathrm{C}}$ resultará infinita cumpliendo el objetivo buscado (SPINELLI 2003b).

Existe un problema práctico al utilizar $G=1$. En estas condiciones, no circulará corriente alguna sobre los resistores $R_{\mathrm{P}}$, es decir tampoco las corrientes de polarización necesarias para el funcionamiento de los A.O. Si las entradas están acopladas en AC mediante capacitores (ver Fig. 6.16), estas corrientes no encontrarán un camino de DC a masa y se integrarán en los capacitores de acoplamiento, llevando al A.B. fuera de su rango de operación ${ }^{13}$.

Dado que en aplicaciones biomédicas no se requiere una $Z_{\mathrm{C}}$ elevada en continua sino solo particularmente para $50 \mathrm{~Hz}$., este problema puede solucionarse adoptando una ganancia $G(s)$ de la forma:

$$
G(s)=\frac{s \tau}{1+s \tau}
$$

Así, la ganancia será nula en DC y, eligiendo adecuadamente la constante de tiempo $\tau$, resultará prácticamente unitaria en $50 \mathrm{~Hz}$. La nueva $Z_{\mathrm{C}}$ está dada por:

$$
Z_{\mathrm{C}}(s)=R_{\mathrm{P}}+R_{\mathrm{P}} \cdot \tau \cdot s
$$

Se observa que ahora $Z_{\mathrm{C}}$ presenta una componente inductiva que, utilizando $R_{\mathrm{P}}=10 \mathrm{M} \Omega$ y constantes de tiempo $\tau$ del orden de los segundos, puede representar inductancias equivalentes del orden de los millones de Henrios i!!!. Esto puede aprovecharse para producir una resonancia paralelo a $50 \mathrm{~Hz}$ entre esta inductancia y las pequeñas capacidades de entrada $C_{\mathrm{IN}}$ propias del amplificador. La frecuencia de resonancia estará dada por:

$$
f_{0}=\frac{1}{2 \pi \sqrt{C_{\mathrm{IN}} \cdot R_{\mathrm{P}} \cdot \tau}}
$$

\footnotetext{
${ }^{13}$ Experimentalmente se observa que "a veces" el amplificador funciona, por momentos llega a saturarse y luego al tocar con "el dedo" se descargan las capacidades y vuelve a funcionar, muchas veces durante tiempos suficientemente largos como para alentar esperanzas.
} 
Un circuito práctico para implementar la topología de la Fig. 6.14 se muestra en la Fig.6.16. Este circuito representa una mejora sobre el bootstrap clásico basado en buffers independientes en cada entrada (PALLAS-ARENY, 1990). La técnica de "bootstrap de modo común" aquí propuesta, proporciona elevadas $\mathrm{Z}_{\mathrm{C}} \mathrm{y}$ permite asignar altas ganancias en el front-end, mientras que las soluciones clásicas obligan a una primer etapa de ganancia unitaria. Una breve descripción del bootstrap clásico se incluye en el APÉNDICE 10.

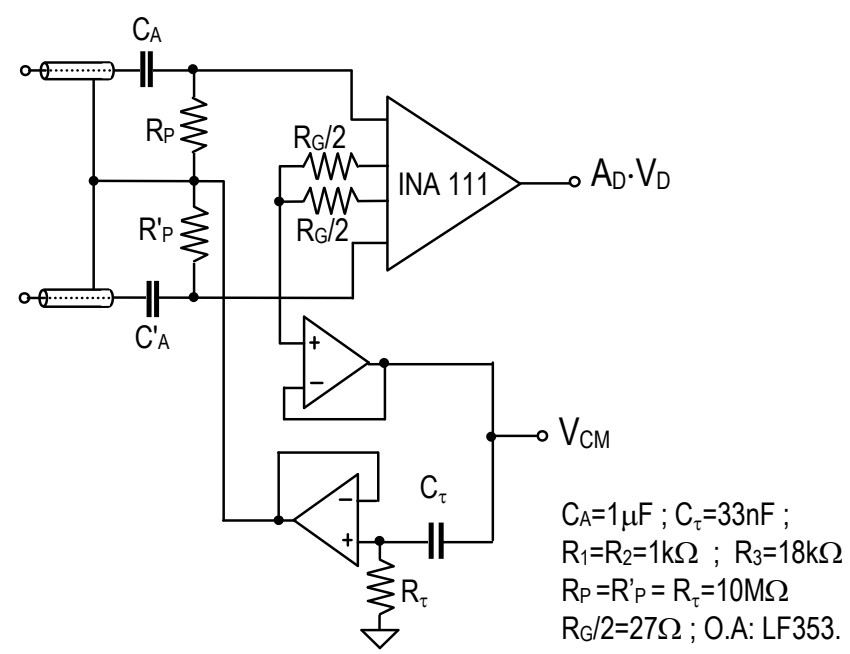

Figura 6.16. Circuito práctico que permite implementar la estrategia propuesta.

\subsubsection{Estimación de la tensión de modo común.}

En el análisis previo, que considera impedancias de generador nulas, la tensión de modo común a la entrada del amplificador $V_{\text {ic }}$ coincide con la tensión de modo común del generador de señal. Esto no es necesariamente así si se consideran las impedancias de los electrodos. En aplicaciones biomédicas, la tensión de modo común del generador de señal $V_{\mathrm{CM}}$, definida sobre la impedancia del electrodo de masa $\mathrm{Z}_{\mathrm{E} 3}$ (ver Fig. 6.1) no es accesible, dado que está definida antes de las impedancias de los electrodos.

Como se mostrará a continuación, para el caso particular del bootstrap de modo común propuesto, la semisuma de las tensiones en sus entradas, es decir $V_{\mathrm{iC}}$, coincide con $V_{\mathrm{CM}}$.

En la Fig.6.17 se muestra un esquema que ahora incorpora las impedancias de electrodo $Z_{\mathrm{E} 1}$ y $Z_{\mathrm{E} 2}$ (estas impedancias incluyen también los capacitores de acoplamiento). En este esquema, las impedancias $Z_{\mathrm{P} 1}, Z_{\mathrm{P} 2}$ corresponden a las resistencias de polarización $R_{\mathrm{P}}$ y a las capacidades de los cables blindados. Este modelo representa la realimentación de modo común mediante el generador $V_{\mathrm{A}} \mathrm{y}$ considera que las impedancias de entrada del amplificador son infinitas. 


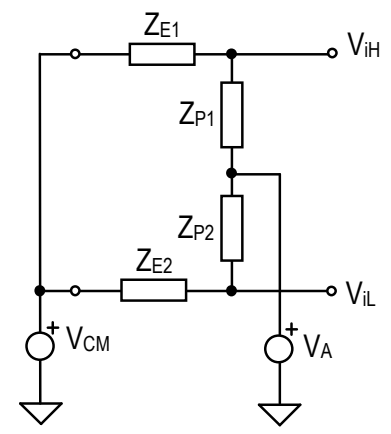

Figura 6.17. Esquema de la realimentación propuesta considerando las impedancias de los electrodos.

Las ecuaciones que permiten resolver este circuito son:

$$
\begin{aligned}
& V_{\mathrm{iH}}=\left(V_{\mathrm{CM}}-V_{\mathrm{A}}\right) \frac{Z_{\mathrm{P} 1}}{Z_{\mathrm{E} 1}+Z_{\mathrm{P} 1}}+V_{\mathrm{A}} \\
& V_{\mathrm{iL}}=\left(V_{\mathrm{CM}}-V_{A}\right) \frac{Z_{\mathrm{P} 2}}{Z_{\mathrm{E} 2}+Z_{\mathrm{P} 2}}+V_{\mathrm{A}}
\end{aligned}
$$

Si se adopta,

$$
V_{\mathrm{A}}=V_{\mathrm{iC}}=\frac{V_{\mathrm{iH}}+V_{\mathrm{iL}}}{2},
$$

reemplazando (6.36) en (6.37), resulta:

$$
V_{\mathrm{A}}=\left(V_{\mathrm{CM}}-V_{\mathrm{A}}\right)\left(\frac{Z_{\mathrm{P} 1}}{Z_{\mathrm{E} 1}+Z_{\mathrm{P} 1}}+\frac{Z_{\mathrm{P} 2}}{Z_{\mathrm{E} 2}+Z_{\mathrm{P} 2}}\right)+V_{\mathrm{A}}
$$

Esta ecuación tiene una única solución que es:

$$
V_{\mathrm{A}}=V_{\mathrm{iC}}=V_{\mathrm{CM}}
$$

Es decir, que realimentando en forma unitaria, la tensión $V_{\mathrm{A}}$ calculada como la semisuma de $V_{\mathrm{iH}}$ y $V_{\mathrm{iL}}$ coincide con la tensión de modo común $V_{\mathrm{CM}}$ del generador independientemente de los desbalances que pudieran existir entre las impedancias. Entonces, como se mostró anteriormente, no circulará corriente de modo común alguna por $Z_{\mathrm{P} 1}, Z_{\mathrm{P} 2}$ y la $Z_{\mathrm{C}}$ de esta red será infinita. Los resistores de polarización $R_{\mathrm{P}}$ no degradan el CMRR.

\subsubsection{Resultados Experimentales.}

La estrategia propuesta se implementó utilizando el circuito de la Fig.6.16. La capacidad de entrada del amplificador incluyendo las propias del circuito impreso resultaron de alrededor de $5 \mathrm{pF}$. Para conseguir una resonancia en $40 \mathrm{~Hz}$ (a fin de 
asegurar una $\mathrm{Z}_{\mathrm{C}}$ capacitiva ${ }^{14}$ a $50 \mathrm{~Hz}$ ), utilizando $R_{\mathrm{P}}=10 \mathrm{M} \Omega$ resulta según (6.35) un valor de $\tau$ de 0.31 segundos. La realimentación de modo común también se utilizó como S-D, para desafectar las capacidades propias del cable blindado utilizado.

En la Fig.6.18 se muestra la impedancia de modo común medida incluyendo cables blindados $\left(C_{\mathrm{SH}} \approx 200 \mathrm{pF}\right)$. Se observa la resonancia deseada alrededor de $40 \mathrm{~Hz}$ y cómo, para frecuencias mayores, $\mathrm{Z}_{\mathrm{C}}$ se aproxima a la correspondiente a la capacidad de entrada propia del amplificador (la impedancia correspondiente a una capacidad $C_{\mathrm{IN}}=5.2 \mathrm{pF}$ esta indicada en línea punteada). La impedancia de modo común a $50 \mathrm{~Hz}$ resultó de unos $700 \mathrm{M} \Omega(800 \mathrm{M} \Omega / / 2 \mathrm{pF})$.

En esta prueba se utilizó cable blindado para aplicaciones de "audio", que siendo liviano y flexible resulta una alternativa de bajo costo para señales biomédicas, evitando recurrir a cables especiales. Este tipo de cable presenta pérdidas moderadas y no produce una marcada resonancia como sí pudo observarse utilizando cables tipo RG-58.

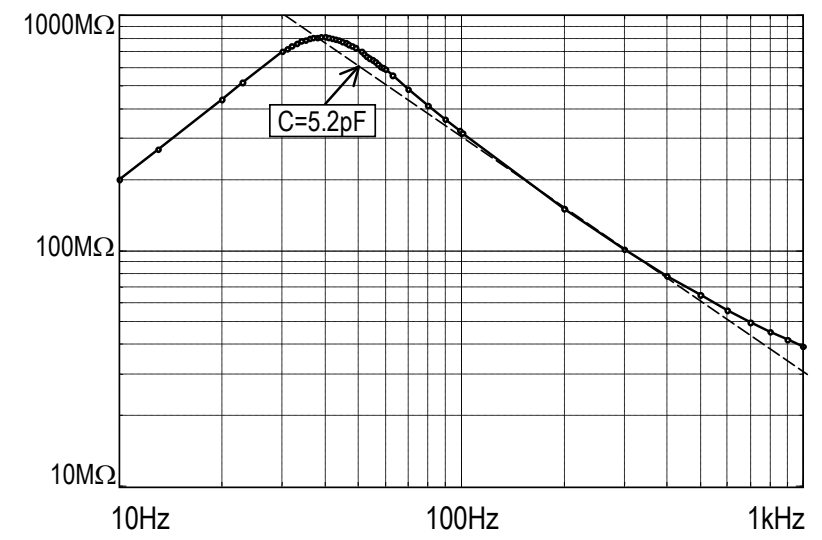

Figura 6.18. Impedancia de entrada Zc del amplificador de la Fig. 6.16 obtenida en forma experimental.

\section{Amplificadores para operar con "Fuente Simple".}

Los amplificadores para Biopotenciales requieren ser alimentados con una fuente simétrica $( \pm)$ a fin de admitir señales de entrada sin polaridad definida respecto a su común (masa). Esto genera un problema, o al menos una incomodidad, cuando el amplificador debe ser alimentado mediante baterías.

Dado que siempre es deseable una operación a partir de una única batería, es usual obtener fuentes duales mediante fuentes conmutadas, las cuales suelen incorporar ruido de conmutación y elevan el nivel de consumo. A continuación, se analizan y presentan estrategias para conseguir operar un A.B. a partir de una única batería (Fuente Simple) sin utilizar convertidores DC/DC.

\footnotetext{
${ }^{14}$ En el CAPítUlo 3 se mostró que una $Z_{\mathrm{C}}$ capacitiva es muy conveniente para sistemas de dos electrodos.
} 
Si el amplificador está alimentado con una única fuente $\left(0-V_{\mathrm{CC}}\right)$ es necesario polarizar sus entradas, por ejemplo a $V_{\mathrm{CC}} / 2$, para que operen con una adecuada tensión de modo común. Existen al menos 2 técnicas para esto: asegurar una apropiada tensión de modo común de DC a la entrada del A.B. o acoplar en alterna la señal de entrada y fijar un nuevo potencial de continua en las entradas del amplificador. A continuación se analizan estas dos posibilidades.

\subsection{Acoplamiento en alterna + Tensión de polarización en DC.}

\subsubsection{Solución Clásica.}

La solución más clásica y a la vez simple se muestra en la Fig.6.19. Los capacitores $C_{1}, C^{\prime}{ }_{1}$, bloquean la tensión de continua de entrada y el generador $V_{\mathrm{POL}}$, a través de $R_{1}, R_{1}^{\prime}$ y $R_{2}$ fija el potencial de continua de las entradas del amplificador.

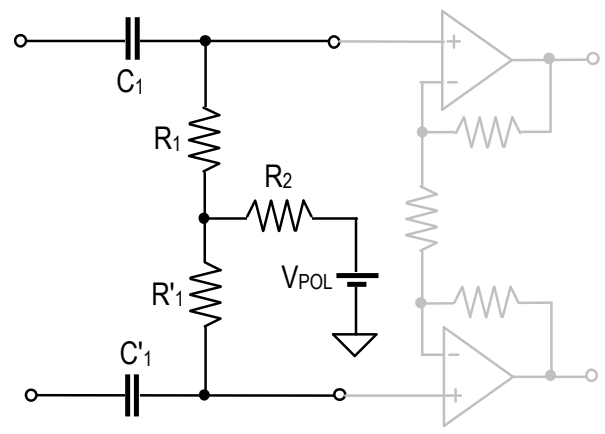

Figura 6.19. Técnica de polarización clásica para entradas de tipo diferencial.

El inconveniente de esta solución es que la red de resistores degrada la impedancia de entrada de modo común. Esto, como se analizó anteriormente, debido al "efecto divisor de potencial" reduce significativamente el rechazo del sistema a interferencias de modo común.

\subsubsection{Una solución propuesta.}

El inconveniente de la degradación de $Z_{\mathrm{C}}$ en la solución clásica, también puede subsanarse utilizando una realimentación de modo común. Se propone aquí una pequeña modificación sobre el bootstrap de modo común introducido en la sección previa, que permite fijar una tensión de polarización de continua, preservando una elevada $Z_{\mathrm{C}}$. Basta con conectar el resistor $R_{\tau}$ de la Fig.6.16 al potencial de continua deseado tal como se muestra en la Fig.6.20.

El comportamiento del circuito en alterna permanece inalterado y como se mostró anteriormente, pueden conseguirse impedancias $Z_{\mathrm{C}}$ muy altas, de hasta algún G $\Omega$. En cuanto al funcionamiento en $\mathrm{DC}$, el potencial del punto medio entre $R_{\mathrm{P}}$ y $R^{\prime}$ P queda fijado en $V_{\mathrm{POL}}$, asegurando la correcta operación del amplificador. Este circuito es apto para sistemas de dos o de tres electrodos (SPINELLI, 2003b), pues las corrientes de polarización no circulan a través del paciente. 


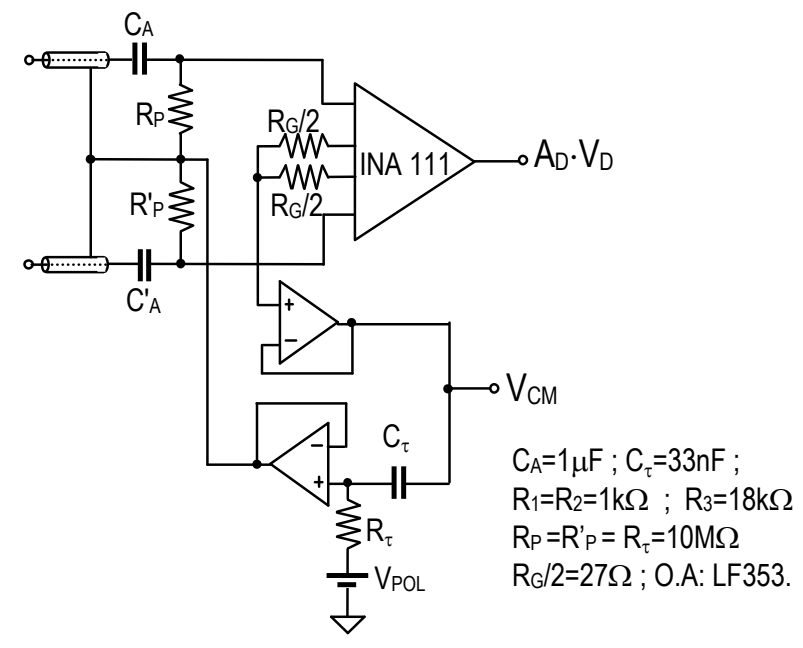

Figura 6.20.Circuito propuesto que permite evitar la degradación de la impedancia de entrada de modo común debida a los resistores de polarización.

\subsection{Control de la tensión de Modo Común de Entrada.}

Otra forma de conseguir polarizar adecuadamente las entradas del A.B., que se propone en esta tesis, consiste en utilizar un control a lazo cerrado de la tensión de modo común. Si se observa algunas secciones atrás (sección 2, pag.110), ésta es precisamente la función de circuito DRL analizado previamente.

En la aplicación clásica, la referencia del circuito DRL como sistema de lazo cerrado se fija a 0 Volt, pues el objetivo es reducir todo lo posible la tensión de modo común $V_{\mathrm{CM}}$. Si se desea que $V_{\mathrm{CM}}$ se mantenga igual a $V_{\mathrm{POL}}$, basta simplemente con conectar la entrada no inversora del amplificador de modo común $A(\mathrm{~s})$ al potencial $V_{\mathrm{POL}}$ como se indica en la Fig.6.21.

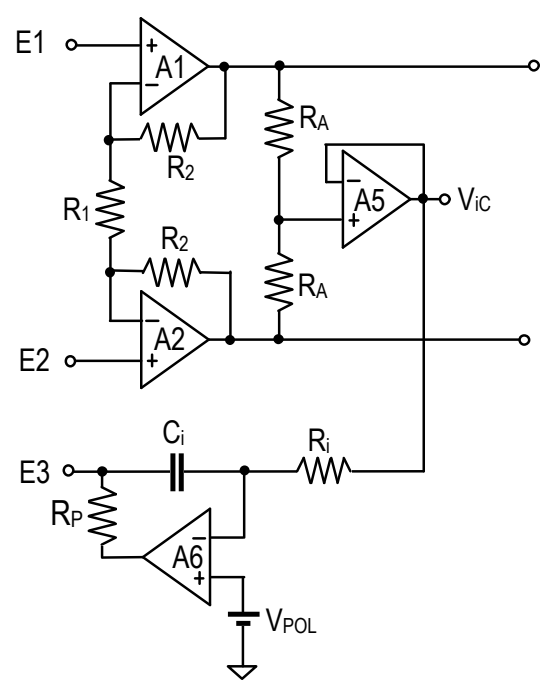

Figura 6.21. Circuito propuesto para fijar el potencial de DC de modo común operando sobre la tensión de referencia del circuito DRL. 
Esta estrategia, tan simple como efectiva, ha sido utilizada satisfactoriamente en muchos de los amplificadores construidos a lo largo de esta tesis (SPINELLI, 2001).. Como puede observarse en la Fig.6.21, salvo la tensión de referencia, nada se ha modificado en el circuito DRL previo, y por lo tanto mantiene su validez el análisis de estabilidad de la sección 2.1.

\section{Conclusiones.}

La realimentación de la tensión de modo común, permite mejorar sensiblemente las características de un A.B.

Mediante esta técnica, puede reducirse la tensión de modo común utilizando el circuito DRL, el cual permite incrementar el rechazo a la interferencia debida a $V_{\mathrm{CM}}$. Este mismo circuito, permite asegurar una tensión de polarización adecuada para operar el A.B. con una fuente simple.

La degradación de la impedancia de entrada de modo común $Z_{\mathrm{C}}$, impuesta por el uso de cables blindados, puede evitarse utilizando un circuito "Shield-Driver" (S-D). Para asegurar la estabilidad de este circuito, es importante utilizar amplificadores operacionales especialmente preparados para operar con ganancia unitaria (con un buen Margen de Fase).

También es posible, mediante la realimentación de modo común, controlar la impedancia de entrada $Z_{\mathrm{C}}$ para señales de modo común. Esto permite conseguir tanto muy bajas $(\approx 100 \Omega)$ como muy altas $(\approx 1 \mathrm{G} \Omega)$ impedancias $Z_{\mathrm{C}}$ manteniendo inalterada la impedancia de entrada $Z_{\mathrm{D}}$ para tensiones de modo diferencial. 
Capítulo 7 - Mejora de la Etapa de Entrada de un A.B. mediante Realimentación de Modo Común 


\section{Capítulo 8: Conclusiones.}

\section{Conclusiones.}

En la primer parte de la tesis se realizó un análisis detallado de los distintos mecanismos a través de los cuales las fuentes de EMI, en especial la tensión de red $(50 \mathrm{~Hz})$, interfieren en el registro de biopotenciales. Esto permitió determinar los requerimientos más importantes para un A.B. que establecieron el punto de partida de la segunda parte, donde los esfuerzos se focalizaron en el diseño de amplificadores y de sus sistemas accesorios. Se propusieron allí distintas soluciones para conseguir bajos niveles de interferencia y ruido, es decir una alta relación Señal-Ruido (SNR).

\subsection{Análisis de EMI en Amplificadores de Biopotenciales.}

Se presentó un modelo simple que describe analíticamente la interferencia de la red $(50 \mathrm{~Hz})$ debida a distintos efectos, permitiendo de este modo estimar los niveles de interferencia esperables en casos típicos, tales como un amplificador de reducidas dimensiones o un amplificador multicanal alimentado mediante un transformador de aislación. La principal utilidad de disponer de expresiones analíticas es que las mismas permiten plantear soluciones para reducir los efectos de EMI, estableciendo las características deseables de un A.B.

El modelo de EMI, es decir la dependencia del nivel de interferencia con los distintos elementos (capacidades de acoplamiento, impedancias de electrodo, etc.), fue validado experimentalmente. Para esto se desarrollaron especialmente métodos que permitieron determinar los parámetros del modelo en condiciones reales de operación. 


\subsubsection{Sistemas de tres electrodos.}

Para el caso de mediciones con "tres electrodos" (con electrodo de masa), los mecanismos de EMI dominantes son el acoplamiento a los cables de conexión y el efecto conocido como "divisor de potencial". Para eliminar el primero basta con utilizar cables blindados, mientras que para reducir el segundo se requieren elevadas impedancias de entrada de modo común $Z_{\mathrm{C}}$ y una baja tensión de modo común $V_{\mathrm{CM}}$.

En estos sistemas, el CMRR del amplificador no es un requerimiento crítico, y aun para las aplicaciones más sensibles un CMRR de $100 \mathrm{~dB}$ resulta suficiente. Si bien este nivel de CMRR requiere un diseño cuidadoso, en esta tesis se mostró que pueden alcanzarse utilizando dispositivos de propósitos generales y sin requerir ajustes en el circuito.

\subsubsection{Sistemas de dos electrodos.}

En los sistemas de adquisición de biopotenciales de "dos electrodos" (sin electrodo de masa), el nivel de interferencia introducido por la red de distribución $(50 \mathrm{~Hz})$ es función de la impedancia de entrada $Z_{C}$ que el A.B. presenta para tensiones de modo común.

Se demostró y se comprobó experimentalmente, que el menor nivel de interferencia se produce para valores extremos de $Z_{C}$, es decir una $Z_{C}$ nula o infinita. La conveniencia de una $u$ otra opción depende del desbalance $\Delta Z_{\mathrm{E}}$ existente entre las impedancias de los electrodos. Es posible definir un valor de desbalance crítico $\Delta Z_{\mathrm{E} . \text { Critico }}$, función del CMRR del A.B. y de las capacidades de acoplamiento $C_{\mathrm{B}}$ y $C_{\mathrm{ISO}}$, que determina cual de las alternativas es la más adecuada para un caso particular.

Para desbalances menores que $\Delta Z_{\text {E.Critico }}$, una $Z_{\mathrm{C}}$ nula conduce al menor nivel de EMI posible para las condiciones dadas, mientras que si el desbalance supera este valor, la menor interferencia se alcanza con una $Z_{\mathrm{C}}$ tan alta como sea posible (idealmente infinita)

Los resultados de este análisis previenen que deben evitarse valores elevados, tanto resistivos como inductivos de $Z_{\mathrm{C}}$, porque las mismas pueden conducir a imprevistamente altos niveles de interferencia.

Por otra parte, en condiciones típicas de medida, la dependencia del rechazo a la tensión de red con $Z_{\mathrm{C}}$ se vuelve significativa para valores de $Z_{\mathrm{C}}$ tan elevados (centenas de $M \Omega$ ), que resultan difíciles de alcanzar. Se podría decir que, usualmente, una alta $Z_{\mathrm{C}}$ o una baja $Z_{\mathrm{C}}$, conducen a similares niveles de rechazo de la interferencia de red. Una $Z_{\mathrm{C}}$ baja tiene la ventaja de fijar reducidas tensiones de modo común, preservando así el rango de entrada del A.B. y convirtiéndose con esto en una alternativa atractiva y actual para mediciones de dos electrodos. 


\subsection{Diseño de Amplificadores para Biopotenciales.}

Un A.B. para sistemas de tres electrodos, debe presentar una elevada $Z_{C} y$ un alto CMRR al mismo tiempo que un bajo nivel de ruido. Estas condiciones son simples de cumplir siempre y cuando sea posible asignar una ganancia importante a la primer etapa. En aplicaciones biomédicas, los potenciales de DC que se producen en la interfase electrodo-piel limitan la máxima ganancia admisible para una etapa de entrada acoplada en DC. Por este motivo, el principal problema de diseño de un A.B. es bloquear las componentes de DC, es decir "acoplar en alterna" la entrada del amplificador sin degradar su impedancia de entrada de modo común ni su CMRR.

Si se dispone de una tensión de alimentación simétrica considerable $( \pm 15 \mathrm{~V})$ y si no existen restricciones en cuanto a consumo y número de componentes, las soluciones clásicas son apropiadas y suficientes para implementar amplificadores de biopotenciales. "Soluciones estándar para problemas estándar"

Por otra parte, para sistemas alimentados a batería, con tensiones de alimentación reducida, los circuitos clásicos conducen a bajos CMRR, niveles de ruido significativos y bajas $Z_{C}$. Como solucinoes a este problema se propusieron en esta tesis, y se evaluaron experimentalmente, nuevos circuitos tanto activos como pasivos que permiten eliminar las componentes de DC antes que éstas ingresen al amplificador, conservando además altos CMRR y elevadas $Z_{C}$.

En cuanto al diseño de A.B. para sistemas de "dos electrodos", se propuso un nuevo circuito que permite conseguir bajas $Z_{C}$ manteniendo altas impedancias de modo diferencial $Z_{D}$. El esquema propuesto, que se basa en una realimentación de modo común, es muy simple y no requiere componentes apareados.

Se estudiaron también distintas aplicaciones de la realimentación de la tensión de modo común $V_{\mathrm{CM}}$ que permiten mejorar notablemente las características de un sistema de adquisición de biopotenciales. Se analizaron en detalle circuitos clásicos como el DRL y el Shield-Driver. Se determinaron las condiciones de estabilidad para cada uno de ellos y también para el caso en que operen en forma conjunta.

Se propusieron nuevas aplicaciones de la realimentación de $V_{\mathrm{CM}}$ como el "bootstrap de modo común" para conseguir muy altas $Z_{\mathrm{C}}$ y la utilización del circuito DRL para polarizar las entradas del A.B. Esta última técnica permite asegurar una adecuada tensión de DC de modo común para la operación de los circuitos a partir de una fuente simple, evitando así el uso de fuentes partidas.

En esta tesis, también se estudiaron los sistemas de adquisición multicanal en configuración "monopolar". Se analizaron sus características propias en cuanto a ruido y rechazo a EMI y se propuso un nuevo amplificador de bajo ruido apropiado para este tipo de aplicaciones. 


\section{Aportes de esta Tesis.}

La presente tesis contiene los siguientes aportes originales:

- Una red pasiva con entrada y salida diferenciales (F-D) para Acoplamiento en Alterna de Amplificadores para Biopotenciales (SPINELLI, 2003.a).

- Un Amplificador para Biopotenciales F-D con supresión de DC. (SPINELLI, 2004.a).

- Un Amplificador para Biopotenciales acoplado en alterna. El mismo emplea bootstraping de modo común para evitar la degradación de $\mathrm{Z}_{\mathrm{C}}$ al incluir resistores de polarización. (SPINELLI, 2003.b).

- Determinación de las condiciones óptimas de medida para sistemas de dos electrodos (SPINELLI, 2004.b)

- Métodos experimentales para medición de las capacidades de acoplamiento $C_{\mathrm{P}}, C_{\mathrm{B}}, C_{\mathrm{SUP}}$ y $C_{\mathrm{ISO}}$; para la medición del desbalance $\Delta \mathrm{Z}_{\mathrm{E}}$ entre dos impedancias electrodo-piel y para la determinación de la impedancia $Z_{C}$ de un A.B.

- Determinación de las condiciones de estabilidad del Circuito ShieldDriver.

- Una técnica para alimentar Amplificadores de Biopotenciales mediante una única fuente simple, basada en el control a lazo cerrado del nivel de DC de la tensión de modo común de entrada. (SPINELLI, 2001).

\section{Líneas Abiertas}

Se planea continuar trabajando sobre los temas desarrollados en esta tesis, en particular en sistemas de adquisición de biopotenciales embebidos en prendas de vestir y en la captura de biopotenciales "sin contacto".

También se prevé extender el uso de circuitos F-D a otras etapas del sistema además del A.B., por ejemplo en etapas de filtrado. 


\subsection{Sistemas de adquisición embebidos en prendas de vestir.}

En algunas aplicaciones, como el monitoreo de pacientes desarrollando sus actividades normales (Holter) o realizando ejercicios controlados (Ergometría), resulta una alternativa atractiva incluir el sistema de captura de biopotenciales en una prenda de vestir. La principal dificultad en la implementación de este tipo de sistema, es conseguir que los electrodos queden operativos al colocar la prenda, es decir sin requerir instalación alguna. Una alternativa que se planea explorar es "embeber" los electrodos en la prenda misma, por ejemplo, utilizando electrodos de hilos metálicos tejidos en la ropa (PARADISO, 2003)

\subsection{Medición sin contacto.}

Los principales inconvenientes que debe afrontar el A.B. se deben a la interfase electrodo-piel. Recientemente han sido propuestos métodos para el registro de biopotenciales que no requieren un contacto galvánico sino que utilizan exclusivamente acoplamiento capacitivo (PRANCE, 2000), (HARLAND, 2002).

Mediante estas técnicas, es posible capturar biopotenciales aún a través de una membrana dieléctrica, resultando robustas frente a los fenómenos que ocurren en la interfase electrodo piel. Por otra parte, dadas las pequeñas capacidades que vinculan al paciente con la entrada del A.B., se requieren amplificadores de muy alta impedancia de entrada tanto para altas como para bajas frecuencias, lo cual exige muy bajas capacidades de entrada. Esto último plantea nuevos desafíos de diseño.

El principal interés de estudiar esta técnica, es utilizarla en sistemas como los descriptos en el punto previo para implementar prendas de vestir con electrodos que no requieran el agregado de gel conductor y al mismo tiempo robustas a artefactos de movimiento.

\subsection{Filtros con entrada y salida diferencial (F-D)}

Como se mostró en esta tesis, los circuitos F-D son muy apropiados para implementar etapas analógicas que requieran altos CMRR, resultando además indicados para circuitos alimentados mediante fuente simple.

En esta tesis, se diseñaron amplificadores respetando esta topología y como línea futura se prevé extender la aplicación de este tipo de circuito a distintas etapas del sistema de adquisición de biopotenciales, en particular en la implementación de filtros.

Los filtros F-D pueden presentar problemas de estabilidad que no se producen en sus contrapartes S-E (CASAS, 1996). En cuanto a este punto, se planea analizar las condiciones de estabilidad y explorar nuevas técnicas de diseño. 
Capítulo 8 - Conclusiones 


\section{Referencias}

AAMI, (1998). American National Standard ANSI/AAMI EC38, 1998: Ambulatory Electrocardiographs. Arlington (VA), Association for the Advancement of Medical Instrumentation, 1999.

Benedetti M., Calcoen D., Fernandez Rovira J., Kloster W., Lorenzo J.M., PETROCHELli R., Uicich G., (2000). "Control de la Interferencia Electromagnética", AADECA, ISBN 950-99994-2-3. Buenos Aires, Argentina.

BOGNER R.E, (1975). "Generalized derivation of the changes in pole positions with respect to variations in the filter coefficients" Capítulo 10 en "Introduction to Digital Filtering”, John Wiley \& Sons, Great Britain, ISBN 0471085901, pp. 181.

BuRKE M.J. and GLEeSON D.T., (2000), "A Micro-power Dry-Electrode ECG Preamplifier," IEEE Transactions on Biomedical Engineering, Vol. 47, pp. 155-162, Feb. 2000.

CASAs O. and PAllas-ARENy R, (1996). "Basics of Analog Differential Filters". IEEE Transactions on Instrumentation and Measurement, Vol. 45, No. 1, pp. 275-279, February 1996.

Christiansen C.F., BAtaiotto P.E. y VAlla M.I., "Análisis comparativo de estructuras diferenciales con amplificadores operacionales", Revista Telegráfica Electrónica, Mayo 1982, pp.456-459.

CRUMP J.F., CosentinO L.C., (1973). "The relationship between the patient and the clinical electrical environment". Proceedings of the Annual Conference on Medical \& Biological Engineering, Vol. 15, pp. 242.

De Jager P.J.A, Peper A., Metting Van Rijn A.C. and Grimbergen C.A., (1996) "High frequency effects in amplifiers for Biopotential Recordings", Proceedings of the 18th Annual International Conference of the Engineering in Medicine and Biology Society IEEE 1996; pp. 26-27.

DOBREV D. and DASKALOV I., (1998) "Two-electrode telemetric instrument for infant heart rate and apnea monitoring", Medical Engineering \& Physics, Vol. 20, pp. 729734.

DoBREV D. and DASKALOV I., (2002a) "Two-electrode biopotential amplifier with current-driven inputs", Medical \& Biological Engineering \& Computing, Vol. 40, pp. 122-127.

DOBREV D. and DASKALOV I., (2002b) "Two-electrode non-differential biopotential amplifier," Medical \& Biological Engineering \& Computing, Vol. 40, pp. 546-549.

DOBREV D., (2004) "Two-electrode low supply voltage electrocardiogram signal amplifier," Medical and Biological Engineering \& Computing, Vol. 42, pp. 272-276. 
Fernandez Chimeno M. and PALlas-AReny R., (2000). "A Comprehensive Model for Power Line Interference in Biopotential Measurements," IEEE Transactions on Instrumentation and Measurement, Vol. 49, pp. 535-540.

Grimbergen C.A, Metting Van Rijn A.C., Peper A, (1991). "Influence of Isolation on Interference in Bioelectric Recordings," Proceedings of the 13th Annual International Conference of the Engineering in Medicine and Biology Society IEEE Vol. 13, No. 4, pp.1720-1721.

Grimbergen C.A., Metting Van Rijn A.C., PePer A, (1992). "A Method for the measurement of the properties of individual electrode-skin interfaces and the implications of the electrode properties for preamplifier design," Proceedings of the 14th Annual International Conference of the Engineering in Medicine and Biology Society IEEE, Vol.6, pp.2382-2383.

Grimbergen C.A., Metting Van Rijn A.C., Kuiper A.P., Honsbeek R.H, Speijer K. and PEPER A, (1995). "DC rejection and deblocking in multichannel bioelectric recordings," Proceedings of the 17th Annual International Conference of the Engineering in Medicine and Biology Society IEEE, pp.1666-1721.

HAMALAiEN M., (1993). "Magentoencephalography - theory, instrumentation , and applications to noinvasive studies of the working human brain", Reviews of Modern Physics, Vol. 65, No. 65, pp. 413-497.

HAMStRA G.H., PePer A. and Grimbergen G.A., (1984). "Low-power instrumentation amplifier for physiological signals," Medical \& Biological Engineering \& Computing, Vol. 22, pp. 272-274.

HuHTA J.C. WeBSTER J.G, (1973). 60-Hz Interference in Electrocardiography. IEEE Transactions on Biomedical Engineering, Vol. 20, pp.91-101.

Harland, C.J., Clark, T.D. and PranCe R.J., (2002). "Electric potential probes: new directions in the remote sensing of the human body," Measurement Science and Technology, Vol.13, pp.163-169.

KRANTZ S.G. (1999): "Handbook of Complex Analysis”. (Birkhäuser, Boston), p. 77

Kunush C., Spinelli E. y Puleston P. y Felice C., (2003). "Estimación de Impedancias Electrodo-Piel utilizando Técnicas de Identificación Paramétrica". Anales del Congreso SABI 2003.

Mc ADAMS E.T, LACKERMEIER A, JOSSINET J., (1994). "AC Impedance of the Hydrogel-Skin Interface". Proceedings of the 16th Annual International Conference of the Engineering in Medicine and Biology Society IEEE, Vol. 2, pp. $870-871$.

Metting Van Rijn A.C., PePer A. and Grimbergen C.A, (1990.a). "High-quality recording of biolectric events: Part 1 Interference reduction, theory and practice", Medical \& Biological Engineering \& Computing, Vol. 28, pp. 389-397. 
Metting Van Rijn A.C., Peper A. and Grimbergen C.A, (1990.b). "High-quality recording of biolectric events: Part II: a low-noise low-power multichannel amplifier design“, Medical \& Biological Engineering \& Computing, Vol. 29, pp. 433-440.

Metting Van Rijn A.C., PePer A. and Grimbergen C.A, (1991). "The Isolation Mode Rejection Ratio in Biolectric Amplifiers," IEEE Transactions on Biomedical Engineering, Vol. 38, No. 11, pp. 1154-1157.

Metting Van Rijn A.C., Peper A. and Grimbergen C.A, (1994), "Amplifiers for bioelectric events: a design with a minimal number of parts," Medical Biological Engineering \& Computing, Vol. 32, pp. 305-310.

MORRISON R., (1998). "Grounding and Shielding Techiniques," John Willey \& Sons, USA, 1998. ISBN 0-471-24518-6.

OTT H., (1988). "Noise Reduction Techniques in Electronic Systems," John Willey \& Sons, USA, 1988. ISBN 0-471-85068-3.

PALLAS-ARENY R. (1986). "On the Reduction of Interference due Common Mode Voltage in Two-Electrode Biopotential Amplifiers", IEEE Transactions on Biomedical Engineering, Vol. 33, pp. 1043-1046.

PALLAS-ARENY R. (1988). "Interference-Rejection Characteristics of Biopotential Amplifiers: A Comparative Analysis," IEEE Transactions on Biomedical Engineering, Vol. 35, pp. 953-959.

PALlas-AReny R., Colominas J. y Rosell J., (1989.a). “An improved Buffer for Bioelectric Signals," IEEE Transactions on Biomedical Engineering, Vol. 36, pp. 490493.

Pallas-ARENy R., Colominas J., (1989.b). "Differential Mode Interference in Biopotential Amplifiers," IEEE Engineering in Medicine \& Biology Society 11Th. Annual International Conference, pp.1721-1722.

PALlAS-ARENY R.. and Webster J.G., (1990). "Composite Instrumentation Amplifier for Biopotentials," Annals of Biomedical Engineering, Vol. 18, pp. 251-262.

Pallas-AReNy R., Colominas J. (1991.b). "Simple, fat method for patient-body capacitance and power-line electric interference measurement," Medical \& Biological Engineering and Computing, Vol. 29, pp. 561-563.

PALlas-ARENY R., Webster J. (1991.c): "Common-Mode Rejection Ratio for cascaded differential amplifier stages", IEEE Transactions on Instrument and Measurements. Vol. 40, pp. 677-681.

PAllas-ARENy R., Webster J., (1991.d). "Common mode rejection ratio in differential amplifiers," IEEE Transactions on Instrumentation and Measurement, Vol. 40, pp. 669-676.

Pallas-Areny R., Webster J., (1999). “Analog Signal Processing”, New York, Wiley. 
Paradiso R., Gemignani A., Scilingo E.P., De Rossi D. (2003). "Knitted Bioclothes for Health Monitoring," Proceedings of the 25th Annual International Conference of the IEEE Engineering in Medicine and Biology Society. Vol. 4, pp.3720-3723.

Prance R.J., Debray A., Clark T.D., Prance H., Nock M, Harland C. ClipPINGDAlE A. J. (2000), "An ultra-low-noise electrical-potential probe for humanbody scanning,” Measurement Science and Technology, Vol.11, pp.291-297.

Ramos J., Pallas-Areny R. and Tresanchez M., (1999). "Multichannel front-end for low level instrumentation signals," Measurements, Vol. 25, pp. 41-46.

Ramos J. y Pallas-Areny R. (2001). "Amplificadores de ECG: De la Norma al Diseño," Proceedings del XIV Congreso Argentino de Bioingeniería SABI 2001 (en CD).

ROLFE, (1983), "Non-invasive physiological measurements",Vol.2, Londres: Academic Press, 1983, ISBN: 0125934025

Rossel J., Colominas J., Riu P., Pallas-AReny R. and Webster J., (1998). "Skin Impedance from $1 \mathrm{~Hz}$ to $1 \mathrm{MHz}$," IEEE Transactions on Biomedical Engineering, Vol. 35, pp. 649-651.

Serrano E., Gasulla M., Casas O. and Pallas-Areny R. (2003). "Power Line Interference in Ambulatory Biopotential Measurements," Proceedings of the $25 \mathrm{t}^{\mathrm{h}}$ Annual Conference IEEE - BME, pp. 3024-3027.

Silva, F, FERNANDEZ, F \& PALlas-ARENY, R., (1994). "Fluorescent Lights Intererence on High Resolution ECG", Proceedings of the World Congress on Medical Physics and Biomedical Engineering. Rio de Janeiro, Brasil, 1994.

Smit H. W., Verton K. and Grimbergen C.A., (1987). "A Low-cost Multichannel Preamplifier for Physiological Signals," IEEE Trans. Biomed. Eng., Vol. BME-34, No. 4, pp. 307-310.

Spinelli E.M., MARTinez N., MAYOSKY M., (1999). "A Transconductance Driven Rigth Leg Circuit,". IEEE Transactions on Biomedical Engineering, Vol. 46, No. 12, pp.1466-1470.

Spinelli E.M., MAYOSKY M., (2000). "AC-Coupled Three op-amp Biopotential Amplifier with Active DC Supression," IEEE Transactions on Biomedical Engineering, Vol. 47, No. 12, pp. 1616-1619.

SPINELli E.M., MARTinez N., and MAYOSKY M., (2001). "A single-supply biopotential amplifier", Medical Engineering \& Physics, Vol. 23, pp. 235-238.

SPiNelli E.M., PALLAS-ARENY R. and MAYOSKY M., (2003.a). "AC-Coupled FrontEnd for biopotential measurements," IEEE Transactions on Biomedical Engineering, Vol. 50, No. 3, pp. 391-395. 
SPINELLi E.M. y MAYOSKY M., (2003.b). "Acoplamiento en AC de Amplificadores para Biopotenciales," Proceedings del XIV Congreso Argentino de Bioingeniería SABI 2003 (en CD), Córdoba.

Spinelli E.M., Martinez N., MAYOSKy M. and PALlas-Areny R., (2004.a). "A Novel Fully-Differential Biopotential Amplifier with DC Suppression," IEEE Transactions on Biomedical Engineering, Vol. 51, No. 8, pp.1444-1448.

Spinelli E.M., PALlas-Areny R. and MAYOSKy M., (2004.b). "Two-Electrode Biopotential measurements: Power Line Interference Analysis,". Aceptado para su publicación en IEEE Transactions on Biomedical Engineering.

THAKOR N.V., and WeBster J.G. (1980): "Ground-Free ECG Recording with Two Electrodes", IEEE Transactions on Biomedical Engineering, Vol. 27, pp. 699-704.

Van Der Horst M.J., Metting Van Rijn A.C., PePer A. and Grimbergen C.A, (1998), "High frequency effects in amplifiers for Biopotential Recordings", Proceedings of the 20th Annual International Conference of the Engineering in Medicine and Biology Society IEEE, pp. 3309-3312.

WeBSTER J.G. (1992). "Medical Instrumentation Application and Design", Boston, MA: Houghton Mifflin, 1992.

WHITE D.R., (1987). "Phase Compensation of the Three Op Amp Instrumentation Amplifier,", IEEE Transactions on Instrumentation and Measurement, Vol. 36, No. 3, pp. 842-844.

WiNTER B. and WeBster J., (1983). "Driven Rigth-Leg Circuit Design," IEEE Transactions on Biomedical Engineering, Vol. 30, No.1, pp. 62-66.

WiTLOCK B., (1996). "Differential Line Receiver with Common-Mode AC Bootstrapping," U.S. Patent \#5568561.

WoOD D.E., EWINS D.J. and BALACHANDRAN W., (1995). "Comparative analysis of power line interference between two- or tree-electrode biopotential amplifiers', Medical \& Biological Engineering \& Computing, Vol. 33, pp. 63-68.

YAмAмото A., (1998). "Elimination of Power Line Interference in ECG signal Using Inverse Loop,", Proceedings 20th Annual International Conference IEEE/EMBS.

YONCE D., (2000). "Input impedance balancing for ECG sensing," U.S Patent \#6208888. 
Referencias 


\section{Apéndice 1}

\section{Valores esperables de Tensiones de Modo común y de Modo Aislante}

\section{Análisis aproximado}

Para estimar los valores que pueden tomar las tensiones de modo común $V_{\mathrm{CM}} \mathrm{y}$ de modo aislante $V_{\text {ISO }}$, resulta suficiente el circuito simplificado de la Fig.A1.1.

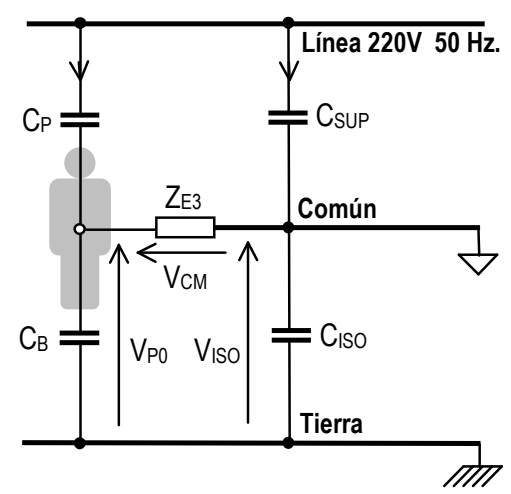

Figura A1.1.Modelo de EMI simplificado, útil para la estimación de la tensión de modo común $\mathrm{V}_{\mathrm{cm}}$.

Las tensiones $V_{\mathrm{CM}}$ y $V_{\text {ISO }}$ son producto de las corrientes de desplazamiento acopladas a través de $C_{\mathrm{P}}$ y $C_{\mathrm{Sup}}$. Salvo un caso muy especial de balance, la corriente sobre una de ellas será dominante en la determinación de los valores de estas tensiones.

\subsection{Caso 1. $C_{p} \gg C_{s u p}$.}

Este caso puede corresponder a un amplificador de tamaño reducido alimentado mediante baterías. Normalmente, el área efectiva del plano de masa de un A.B. de este tipo es reducida y su capacidad $C_{\text {SUP }}$ resulta despreciable. El circuito equivalente para este caso resulta:

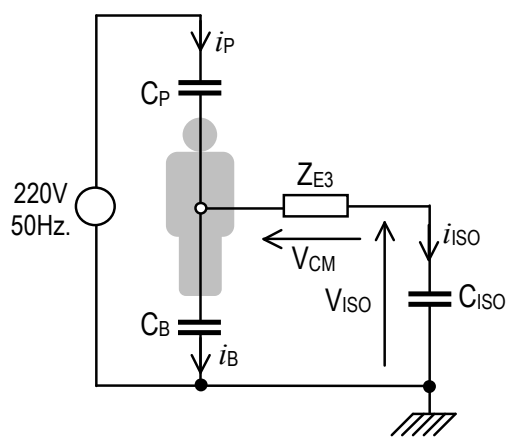

Figura A1.2. Circuito Equivalente considerando CP» Csup 
La corriente de desplazamiento $i_{\mathrm{P}}$ que ingresa al paciente se distribuye entre $i_{\mathrm{B}}$, que circula sobre $C_{\mathrm{B}}$ e $i_{\mathrm{ISO}}$ que lo realiza sobre $C_{\mathrm{ISO}}$. Como $\mathrm{Z}_{\mathrm{E} 3} \ll \mathrm{Z}_{\mathrm{ISO}}$, este reparto está determinado principalmente por la relación $C_{\mathrm{B}} / C_{\mathrm{ISO}}$.

La diferencia de potencial que $i_{\mathrm{ISO}}$ produce sobre $Z_{\mathrm{E} 3}$ fija la tensión de modo común $V_{\mathrm{CM}}$ y la que imprime sobre $C_{\mathrm{ISO}}$ determina de modo aislante $V_{\text {Iso. Estas tensiones }}$ están dadas aproximadamente por:

$$
\begin{aligned}
& V_{\mathrm{CM}} \cong 220 \mathrm{~V} \cdot \omega \cdot C_{\mathrm{P}} \cdot Z_{\mathrm{E} 3} \cdot\left(\frac{C_{\mathrm{ISO}}}{C_{\mathrm{B}}+C_{\mathrm{ISO}}}\right) \\
& V_{\mathrm{ISO}} \cong 220 \mathrm{~V} \cdot \frac{C_{\mathrm{P}}}{C_{\mathrm{B}}+C_{\mathrm{ISO}}}
\end{aligned}
$$

Como puede observarse en (A1.1), si la capacidad $C_{\text {ISo }}$ es nula, también lo será la tensión $V_{\mathrm{CM}}$ : una buena razón para intentar conseguir altos niveles de aislamiento.

Considerando una $C_{\mathrm{ISO}}=30 \mathrm{pF}$ y condiciones desfavorables de medida (pero no poco frecuentes) con altos valores de $C_{\mathrm{P}}$ y $Z_{\mathrm{E} 3}\left(C_{\mathrm{P}}=5 \mathrm{pF}, C_{\mathrm{B}}=100 \mathrm{pF}, Z_{\mathrm{E} 3}=100 \mathrm{k} \Omega\right)$, resulta:

$$
V_{\mathrm{CM}}=7.7 \mathrm{mV} \quad ; \quad V_{\text {ISO }}=8.1 \mathrm{~V}
$$

\subsection{Caso 2. $C_{\text {sup }} \gg C_{p}$.}

Este podría ser el caso de un sistema con un gran número de canales, alimentado mediante un transformador de aislamiento. En este caso, tanto $C_{\mathrm{SUP}}$ como $C_{\mathrm{ISO}}$, pueden tomar valores elevados de varias decenas de $\mathrm{pF}$. Despreciando $Z_{\mathrm{E} 3}$ frente a las demás impedancias involucradas resulta:

$$
\begin{aligned}
& V_{\mathrm{CM}} \cong 220 \mathrm{~V} \cdot \omega_{\mathrm{P}} \cdot C_{\mathrm{SUP}} \cdot Z_{\mathrm{E} 3} \cdot\left(\frac{C_{\mathrm{B}}}{C_{\mathrm{SUP}}+C_{\mathrm{B}}+C_{\mathrm{ISO}}}\right) \\
& V_{\mathrm{ISO}} \cong 220 \mathrm{~V} \cdot \frac{C_{\mathrm{SUP}}}{C_{\mathrm{SUP}}+C_{\mathrm{B}}+C_{\mathrm{ISO}}}
\end{aligned}
$$

Una situación desfavorable implica altas $C_{\mathrm{SUP}}, C_{\mathrm{B}}$ y bajas $Z_{\mathrm{E} 3}$ y $C_{\mathrm{ISO}}$. Por ejemplo, considerando $C_{\mathrm{SUP}}=100 \mathrm{pF}, C_{\mathrm{ISO}}=10 \mathrm{pF}, C_{\mathrm{B}}=300 \mathrm{pF}$ y $Z_{\mathrm{E} 3}=100 \mathrm{k} \Omega$, resulta:

$$
V_{\mathrm{CM}}=506 \mathrm{mV} ; V_{\mathrm{ISO}}=54 \mathrm{~V}
$$

\subsection{Otros efectos.}

En este análisis se supuso que el paciente es isopotencial. En realidad, y tal como se mostró en la Fig.2.1, el cuerpo del paciente presenta impedancias no nulas, y sobre éstas se desarrollan potenciales tanto de modo diferencial como de modo común. 
La tensión de modo común sobre el paciente está dada por la diferencia de potencial que produce $i_{\mathrm{P}}$ sobre la impedancia interna del cuerpo $Z_{\mathrm{P} 2}$ (ver Fig.2.1 del CAPÍTULO 2). Considerando una $i_{\mathrm{P}}$ elevada, de $1 \mu \mathrm{A}$ y una $Z_{\mathrm{P} 2}$ de $500 \Omega$ resultaría una $V_{\mathrm{CM}}$ dada por:

$$
V_{\mathrm{CM}} \cong i_{\mathrm{P}} \cdot Z_{\mathrm{P} 2}=1 \mu \mathrm{A} \cdot 500 \Omega=0.5 \mathrm{mV}
$$

Las impedancias de electrodo también producen tensión de modo común que puede calcularse como:

$$
V_{\mathrm{CM}} \cong\left(i_{\mathrm{L} 1} Z_{\mathrm{E} 1}+i_{\mathrm{L} 2} Z_{\mathrm{E} 2}\right) / 2
$$

Siendo $i_{\mathrm{L} 1}, i_{\mathrm{L} 2}$ las corrientes acopladas a los cables de conexión 1 y 2 respectivamente.

Por ejemplo, si $Z_{\mathrm{E} 1}=Z_{\mathrm{E} 2}=100 \mathrm{k} \Omega$ e $i_{\mathrm{L} 1}=i_{\mathrm{L} 2}=10 \mathrm{nA}$, resulta

$$
V_{\mathrm{CM}} \cong 1 \mathrm{mV}
$$

Como se observa en (A1.5) y (A1.7), estas últimas tensiones de modo común son pequeñas frente a las calculadas en los puntos previos, lo cual justifica la asunción de un paciente isopotencial.

\subsection{Conclusiones.}

Considerando que $C_{\mathrm{P}}$ y $C_{\mathrm{B}}$ son parámetros que escapan al control del diseñador, para que la tensión $V_{\mathrm{CM}}$ sea reducida se requieren bajas capacidades $C_{\mathrm{ISO}}$ y $C_{\mathrm{SUP}}$, es decir fuentes de alimentación con buena aislación y amplificadores de reducido tamaño.

Por otra parte, para reducir $V_{\text {ISO }}$ se requieren bajas $C_{\text {SUP }}$ y/o bajas impedancias de aislación $Z_{\text {ISO }}$ (altas $C_{\text {ISO }}$ ). Esto último implica necesariamente reducir la aislación paciente-tierra, lo cual está limitado, por regulaciones de seguridad a $Z_{\mathrm{ISO}}>22 \mathrm{M} \Omega^{1}$ (AAMI, 1998).

$V_{C M}$ depende de la impedancia $Z_{\mathrm{E} 3}$ del tercer electrodo y de las capacidades de acoplamiento mientras que $V_{\text {ISO }}$ depende exclusivamente de las relaciones entre estas capacidades.

Es importante notar que una alta aislación entre línea y común ( $C_{\text {SUP }}$ pequeñas) reduce al mismo tiempo $V_{\mathrm{CM}}$ y $V_{\text {ISO }}$. Los esfuerzos de diseño deben apuntar a reducir $C_{\mathrm{SUP}}$ todo lo posible, por ejemplo utilizando amplificadores de pequeño tamaño alimentados por baterías.

La tensión $V_{\mathrm{CM}}$ puede llegar a ser de centenas de $\mathrm{mV}$, mientras que $V_{\text {ISo }}$ puede tomar valores mucho mayores, del orden de las decenas de Volts (unos $40 \mathrm{~dB}$ mayor).

\footnotetext{
${ }^{1}$ En caso de ser muy necesario, en (Meeting Van Rijn, et al., 1991) se muestra una forma de reducir la aislación en forma controlada.
} 
Apéndice 1 - Valores esperables de Tensiones de Modo Común y de Modo Aislante 


\section{Apendice 2}

\section{Análisis detallado del "Efecto Divisor de Potencial"}

\section{Efecto "Divisor de Potencial"}

Uno de los problemas más comunes en la adquisición de biopotenciales es la transformación de modo común a modo diferencial debida a desbalances en las impedancias electródicas. Como puede observarse en la Fig.A2.1, despreciando el efecto de la impedancia de entrada de modo diferencial ${ }^{1}$, quedan conformados dos divisores de tensión. Si estos no son idénticos, una tensión de entrada de modo común produce una tensión de modo diferencial que no podrá ser rechazada por un amplificador posterior.

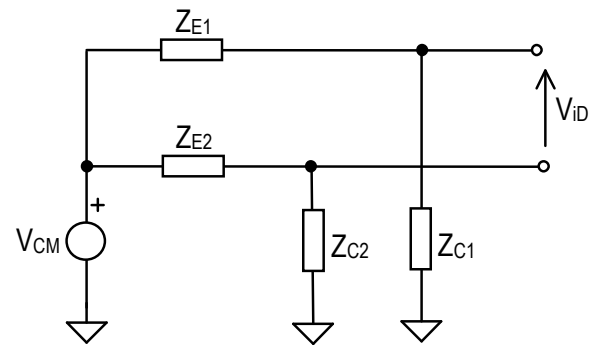

Figura A2.1. Efecto "divisor de potencial". Las impedancias $Z_{\mathrm{E} 1}, \mathrm{Z}_{\mathrm{C} 1}$ y $\mathrm{Z}_{\mathrm{E} 2}, \mathrm{Z}_{\mathrm{C} 2}$, componen, respectivamente, dos divisores de tensión. Si éstos no verifican la misma relación $Z_{\mathrm{Ci}} / Z_{\mathrm{Ei}}$, la tensión de modo común $\mathrm{V}_{\mathrm{CM}}$ produce una tensión de modo diferencial $\mathrm{V}_{\mathrm{iD}}$.

La tensión diferencial $V_{\mathrm{iD}}$ está dada por:

$$
\begin{aligned}
& V_{\mathrm{iD}}=V_{\mathrm{CM}}\left(\frac{Z_{\mathrm{C} 1}}{Z_{\mathrm{C} 1}+Z_{\mathrm{E} 1}}-\frac{Z_{\mathrm{C} 2}}{Z_{\mathrm{C} 2}+Z_{\mathrm{E} 2}}\right) \\
& V_{\mathrm{iD}}=V_{\mathrm{CM}}\left(\frac{1}{1+Z_{\mathrm{E} 1} / Z_{\mathrm{C} 1}}-\frac{1}{1+Z_{\mathrm{E} 2} / Z_{\mathrm{C} 2}}\right)
\end{aligned}
$$

utilizando la aproximación:

$$
\frac{1}{1+x} \cong 1-x \text { si } x<<1
$$

y considerando que $Z_{\mathrm{E}} \ll Z_{\mathrm{C}}$, la ecuación (A2.2) puede llevarse a:

$$
V_{\mathrm{iD}}=V_{\mathrm{CM}}\left[\left(1-Z_{\mathrm{E} 1} / Z_{\mathrm{C} 1}\right)-\left(1-Z_{\mathrm{E} 2} / Z_{\mathrm{C} 2}\right)\right] \quad,
$$

\footnotetext{
${ }^{1}$ En (Pallas Areny, 1999) se demuestra que la impedancia de entrada de modo diferencial no influye en el mecanismo aquí analizado.
} 
o en forma más compacta:

$$
V_{\mathrm{iD}}=V_{\mathrm{CM}}\left(Z_{\mathrm{E} 2} / Z_{\mathrm{C} 2}-Z_{\mathrm{E} 1} / Z_{\mathrm{C} 1}\right)
$$

Si consideramos que $Z_{\mathrm{C} 1}$ y $Z_{\mathrm{C} 2}$ difieren en un $\Delta Z_{\mathrm{C}}$ y $Z_{\mathrm{E} 1}$ y $Z_{\mathrm{E} 2}$ en $\Delta Z_{\mathrm{E}}$, el peor caso es:

$$
V_{\mathrm{iD}}=V_{\mathrm{CM}}\left(\frac{Z_{\mathrm{E}}+\Delta Z_{\mathrm{E}} / 2}{Z_{\mathrm{C}}-\Delta Z_{\mathrm{C}} / 2}-\frac{Z_{\mathrm{E}}-\Delta Z_{\mathrm{E}} / 2}{Z_{\mathrm{C}}+\Delta Z_{\mathrm{C}} / 2}\right)=V_{\mathrm{CM}} \frac{Z_{\mathrm{C}} \Delta Z_{\mathrm{E}}+Z_{\mathrm{E}} \Delta Z_{\mathrm{C}}}{\left(Z_{\mathrm{C}}^{2}-\Delta Z_{\mathrm{C}}^{2} / 4\right)} .
$$

Considerando $\Delta Z_{\mathrm{C}} \ll Z_{\mathrm{C}}$ la expresión (A2.6) puede aproximarse por:

$$
V_{\mathrm{iD}} \cong V_{\mathrm{CM}} \cdot \frac{Z_{\mathrm{C}} \Delta Z_{\mathrm{E}}+Z_{\mathrm{E}} \Delta Z_{\mathrm{C}}}{Z_{\mathrm{C}}^{2}}
$$

obteniendo finalmente:

$$
V_{\mathrm{iD}} \cong V_{\mathrm{CM}} \cdot \frac{Z_{\mathrm{E}}}{Z_{\mathrm{C}}}\left\{\frac{\Delta Z_{\mathrm{E}}}{Z_{\mathrm{E}}}-\frac{\Delta Z_{\mathrm{C}}}{Z_{\mathrm{C}}}\right\}
$$

En general, el desbalance relativo en $Z_{\mathrm{C}}$ es despreciable frente al correspondiente a $Z_{\mathrm{E}}$ y (A2.8) se reduce a:

$$
V_{\mathrm{iD}} \cong V_{\mathrm{CM}} \cdot \frac{\Delta Z_{\mathrm{E}}}{Z_{\mathrm{C}}}
$$




\section{Apéndice 3}

\section{Cálculo de las tensiones de Modo Diferencial y Modo Común en sistemas de 2 electrodos.}

\section{Introducción.}

Para analizar la interferencia de la tensión de red en sistemas de dos electrodos, se requiere de un modelo para las impedancias de entrada del A.B. que permita describir tanto altas como bajas impedancias de modo común $Z_{C}$. En el CAPÍtulo 3 se propusieron allí dos modelos posibles: con realimentación de modo común en corriente (Fig.A3.1.a) y con realimentación de modo común en tensión (Fig.A3.1.b). En este apéndice se determinará, para cada uno de ellos, las tensiones de modo común y de modo diferencial producidas por la tensión de red a la entrada del A.B. El análisis realizado demuestra que ambos modelos conducen a idénticos resultados.

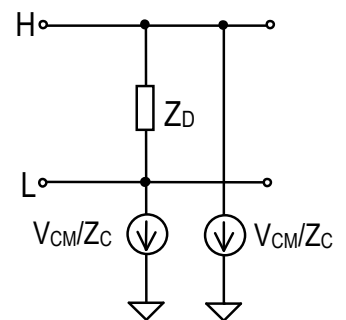

(a)

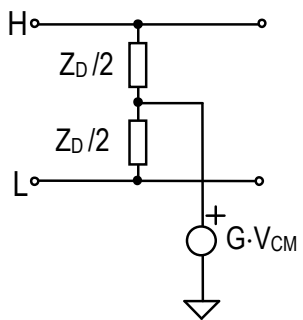

(b)

Figura A3.1.Modelos de Impedancias de Entrada para describir bajas impedancias de modo común. (a) Realimentación en corriente. (b) Realimentación en tensión.

\section{Modelo con realimentación de Tensión de Modo Común.}

El circuito equivalente, correspondiente al modelo de EMI de un sistema de dos electrodos, considerando este modelo de impedancias de entrada de la Fig.A3.1.b, fue presentado en el CAPítULO 3. Por comodidad, se lo repite aquí en la Fig.A3.2.

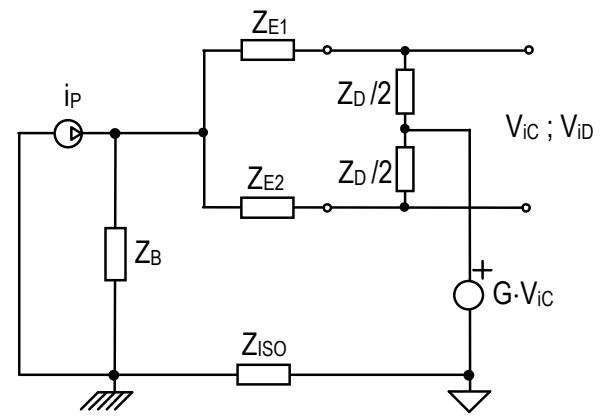

Figura A3.2. Circuito equivalente del modelo de interferencia, que considera el modelo de impedancias de entrada de la Fig.A 3.1.b. 
Aplicando el Teorema de Thevenin, se obtiene un circuito algo más simple, que se muestra en la Fig A3.3.

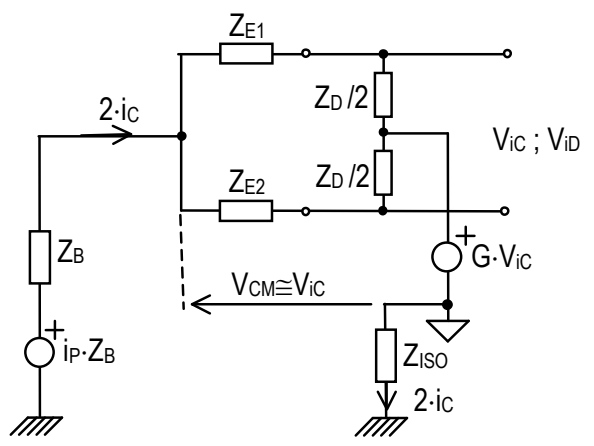

Figura A3.3. Circuito equivalente del modelo de la Fig. A3.2.

\subsection{Impedancia de entrada de modo común.}

Para simplificar la resolución del circuito se calculará en primer lugar el circuito equivalente visto por un generador de modo común $V_{\mathrm{CM}}$, es decir entre el "paciente" y el "común". Si se considera que las impedancias de electrodo $Z_{\mathrm{E} 1}, Z_{\mathrm{E} 2}$ son mucho menores que la impedancia de modo diferencial $Z_{\mathrm{D}}$ (esto debe ser así para no atenuar la tensión bioeléctrica), el circuito equivalente resulta:

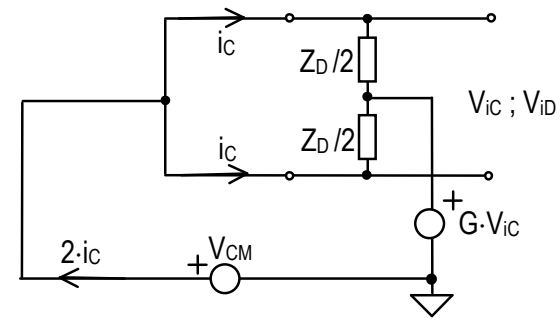

Figura A3.4. Circuito Equivalente entre "paciente" y "común".

Dada la simetría del circuito, la corriente entregada por el generador $V_{\mathrm{CM}}$ se divide en partes iguales por las dos ramas y la corriente $i_{\mathrm{C}}$ por cada una de ellas es ${ }^{1}$ :

$$
i_{\mathrm{C}}=\frac{V_{\mathrm{iC}}(1-G)}{Z_{\mathrm{D}} / 2}
$$

Finalmente, la impedancia de entrada que este circuito presenta para tensiones de modo común $\left(Z_{\mathrm{C}}=V_{\mathrm{iC}} / i_{\mathrm{C}}\right)$, queda dada por:

$$
Z_{\mathrm{C}}=\frac{Z_{\mathrm{D}} / 2}{1-G}
$$

\footnotetext{
${ }^{1}$ Aquí también se utilizó el hecho que, al considerar despreciables las impedancias de los electrodos, la tensión de modo común de entrada $V_{\mathrm{iC}}$ coincide con la tensión de modo común del paciente $V_{\mathrm{CM}}$.
} 


\subsection{Tensiones de modo común y de Modo Diferencial}

Teniendo en cuenta que el sub-circuito de la Fig.A3.4 puede reemplazarse por un impedancia $Z_{\mathrm{C}}$ dada por (A3.2), la corriente total de entrada al amplificador $\left(=2 i_{\mathrm{C}}\right.$ ), puede expresarse como:

$$
2 \cdot i_{\mathrm{C}}=\frac{i_{\mathrm{P}} Z_{\mathrm{B}}}{Z_{\mathrm{B}}+Z_{\mathrm{ISO}}+Z_{\mathrm{C}} / 2}
$$

De donde se obtiene:

$$
i_{\mathrm{C}}=\frac{i_{\mathrm{P}} Z_{\mathrm{B}}}{\left(Z_{\mathrm{B}}+Z_{\mathrm{ISO}}\right) 2+Z_{\mathrm{C}}}
$$

Estas corrientes $i_{\mathrm{C}}$ producirán tensiones de modo común y de modo diferencial de acuerdo a:

$$
\begin{aligned}
& V_{\text {iC }}=i_{\mathrm{C}} Z_{\mathrm{C}} \\
& V_{\mathrm{iD}}=i_{\mathrm{C}} \Delta Z_{\mathrm{E}} \quad .
\end{aligned}
$$

Siendo $\Delta Z_{\mathrm{E}}$ el desbalance entre las impedancias de electrodo definido como $\Delta Z_{\mathrm{E}}=Z_{\mathrm{E} 1^{-}}$ $Z_{\mathrm{E} 2}$. Si se reemplaza (A3.4) en (A3.5) y (A3.6), resultan finalmente:

$$
\begin{aligned}
& V_{\mathrm{iC}}=\frac{i_{\mathrm{P}} \cdot Z_{\mathrm{B}} Z_{\mathrm{C}}}{\left(Z_{\mathrm{B}}+Z_{\mathrm{ISO}}\right) 2+Z_{\mathrm{C}}} \\
& V_{\mathrm{iD}}=\frac{i_{\mathrm{P}} \cdot Z_{\mathrm{B}} \Delta Z_{\mathrm{E}}}{\left(Z_{\mathrm{B}}+Z_{\mathrm{ISO}}\right) 2+Z_{\mathrm{C}}}
\end{aligned}
$$

\section{Modelo con realimentación en corriente (DOBREV, 2002).}

Para este modelo de impedancias de entrada (Fig.A3.1.a), el circuito equivalente correspondiente al modelo de EMI:

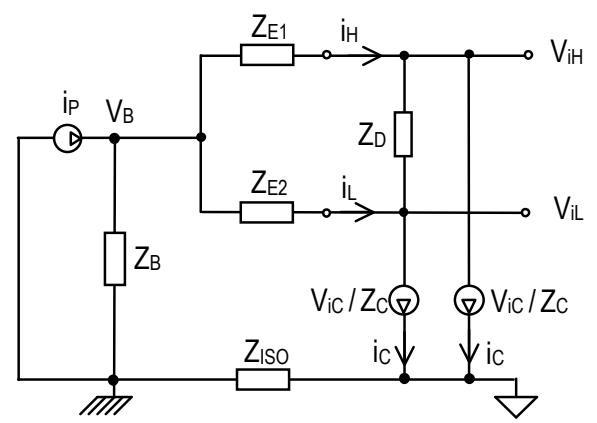

Figura A3.5. Circuito equivalente del modelo de interferencia que considera el modelo de impedancias de entrada de la Fig A3.1.a. 


\subsection{Corrientes a través de los electrodos.}

De acuerdo al circuito de la Fig. A3.4, las corrientes $i_{\mathrm{H},} i_{\mathrm{L}}$, que circulan a través de las impedancias electródicas, están dadas por:

$$
\begin{gathered}
i_{\mathrm{H}}=i_{\mathrm{C}}+\frac{V_{\mathrm{iD}}}{Z_{\mathrm{D}}} \\
i_{\mathrm{L}}=i_{\mathrm{C}}-\frac{V_{\mathrm{iD}}}{Z_{\mathrm{D}}}
\end{gathered}
$$

Estas corrientes, circulando sobre las impedancias de electrodo $Z_{\mathrm{E} 1}$ y $Z_{\mathrm{E} 2}$, producen una tensión de modo diferencial $V_{\mathrm{iD}}$ :

$$
V_{\mathrm{iD}}=i_{\mathrm{L}} Z_{\mathrm{E} 2}-i_{\mathrm{H}} Z_{\mathrm{E} 1}
$$

Restando (A3.9) y (A3.10):

$$
V_{\mathrm{iD}}=\left(i_{\mathrm{L}}-i_{\mathrm{H}}\right) Z_{\mathrm{D}} / 2
$$

y reemplazando (A3.12) en (A3.11), resulta:

$$
i_{\mathrm{H}}\left(Z_{\mathrm{D}} / 2+Z_{\mathrm{E} 1}\right)=i_{\mathrm{L}}\left(Z_{\mathrm{D}} / 2+Z_{\mathrm{E} 2}\right)
$$

que puede escribirse como:

$$
\frac{i_{\mathrm{H}}}{i_{\mathrm{L}}}=\frac{\left(Z_{\mathrm{D}} / 2+Z_{\mathrm{E} 2}\right)}{\left(Z_{\mathrm{D}} / 2+Z_{\mathrm{E} 1}\right)}
$$

Teniendo en cuenta que para evitar la atenuación de la señal bioeléctrica, la impedancia de entrada de modo diferencial $Z_{\mathrm{D}}$ debe ser mucho mayor que las impedancias de los electrodos, a partir de (A3.14) se concluye que las corrientes que circulan por cada uno de los electrodos son muy similares $\left(i_{\mathrm{H}} \cong i_{\mathrm{L}}\right)$. Finalmente,

$$
i_{\mathrm{H}} \cong i_{\mathrm{L}} \cong i_{\mathrm{C}}
$$

\subsection{Tensión de Entrada de Modo Común}

El potencial en los terminales de entrada " $\mathrm{H}$ " $\mathrm{y}$ "L", están dados por:

$$
\begin{aligned}
& V_{\mathrm{iH}}=V_{\mathrm{B}}-i_{\mathrm{H}} Z_{\mathrm{E} 1}, \\
& V_{\mathrm{iL}}=V_{\mathrm{B}}-i_{\mathrm{L}} Z_{\mathrm{E} 2},
\end{aligned}
$$

donde el potencial del cuerpo del paciente $V_{\mathrm{B}}$ puede ser expresado como:

$$
V_{\mathrm{B}}=\left(i_{\mathrm{P}}-2 i_{\mathrm{C}}\right) Z_{\mathrm{B}}-2 i_{\mathrm{C}} Z_{\text {ISO }}=i_{\mathrm{P}} Z_{\mathrm{B}}-2 i_{\mathrm{C}}\left(Z_{\mathrm{B}}+Z_{\text {ISO }}\right)
$$


A partir de (A3.16) y (A3.17) se obtiene la tensión de entrada de modo común $V_{\mathrm{ic}}$ :

$$
V_{\mathrm{iC}}=\left(V_{\mathrm{iH}}+V_{\mathrm{iL}}\right) / 2=V_{\mathrm{B}}-i_{\mathrm{H}} Z_{\mathrm{E} 1} / 2-i_{\mathrm{L}} Z_{\mathrm{E} 2} / 2
$$

y reemplazando (A3.18) y (A3.15) en (A3.19) resulta:

$$
V_{\mathrm{iC}}=i_{\mathrm{P}} Z_{\mathrm{B}}-2 i_{\mathrm{C}}\left(Z_{\mathrm{B}}+Z_{\mathrm{ISO}}\right)-i_{\mathrm{C}}\left(Z_{\mathrm{E} 1}+Z_{\mathrm{E} 2}\right) / 2
$$

Utilizando $i_{\mathrm{C}}=V_{\mathrm{iC}} / Z_{\mathrm{C}}$, esta ecuación se transforma en:

$$
V_{\mathrm{iC}}=i_{\mathrm{P}} \frac{Z_{\mathrm{B}} Z_{\mathrm{C}}}{Z_{\mathrm{C}}+2\left(Z_{\mathrm{B}}+Z_{\mathrm{ISO}}\right)+\left(Z_{\mathrm{E} 1}+Z_{\mathrm{E} 2}\right) / 2}
$$

Teniendo en cuenta que $Z_{\mathrm{E} 1}+Z_{\mathrm{E} 2} \ll Z_{\mathrm{B}}+Z_{\mathrm{ISO}}$, la tensión $V_{\mathrm{iC}}$ dada por (A3.21) puede aproximarse por:

$$
V_{\mathrm{iC}} \cong i_{\mathrm{P}} \frac{Z_{\mathrm{B}} Z_{\mathrm{C}}}{Z_{\mathrm{C}}+2\left(Z_{\mathrm{B}}+Z_{\mathrm{ISO}}\right)}
$$

y las corrientes de entrada de modo común resultan:

$$
i_{\mathrm{C}}=\frac{V_{\mathrm{iC}}}{Z_{\mathrm{C}}} \cong i_{\mathrm{P}} \frac{Z_{\mathrm{B}}}{Z_{\mathrm{C}}+2\left(Z_{\mathrm{B}}+Z_{\mathrm{ISO}}\right)}
$$

\subsection{Tensión Diferencial de Entrada.}

Reemplazando (A3.23) y (A3.15) en (A3.11) se obtiene la tensión diferencial de entrada $V_{\text {iD }}$ dada por:

$$
V_{\mathrm{iD}} \cong i_{\mathrm{P}} \frac{Z_{\mathrm{B}}\left(Z_{\mathrm{E} 2}-Z_{\mathrm{E} 1}\right)}{Z_{\mathrm{C}}+2\left(Z_{\mathrm{B}}+Z_{\mathrm{ISO}}\right)}
$$

Una conclusión importante de esta análisis, que surge de comparar los resultados de los puntos 2 y 3 , es que los dos modelos de impedancias de entrada conducen a idénticas tensiones tanto de modo común como de modo diferencial. Por lo tanto, el análisis presentado en el CAPítulo 3 es válido para ambos tipos de modelos de impedancias de entrada. 
Apéndice 3 - Cálculo de las tensiones de Modo Diferencial y Modo Común en sistemas de 2 electrodos 


\section{Apéndice 4 \\ Simulación de resistencias de valor elevado}

\section{Introducción.}

En algunas aplicaciones se requieren resistencias de elevado valor $(>100 \mathrm{M} \Omega)$, difíciles de conseguir o construir. Un recurso habitual es simularlas mediante circuitos activos, por ejemplo utilizando una red " $T$ " en la realimentación. De este modo, es posible implementar "resistencias equivalentes" elevadas utilizando resistores estándar; pero aquí se manifiesta una vez más el Principio de Conservación de la Dificultad ${ }^{l}$ : estas resistencias equivalentes no se comportan exactamente como un resistor real de igual valor. En este apéndice se analizan estas diferencias.

\section{Simulación de Altas Resistencias.}

Un esquema típico para simular resistencias elevadas es el que se muestra en la Fig.A4.1 (PALlAS-ARENY, 1989.a). Este circuito se comporta, visto desde los terminales " $\mathrm{H}$ " y "L", como un resistor $R_{\mathrm{EQ}} \cong R_{1} \cdot R_{2} / R_{3}$.

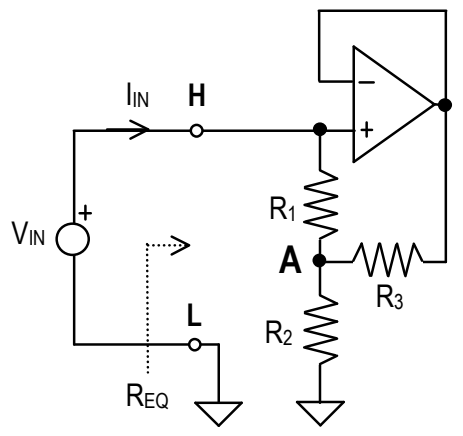

Figura A4.1. Circuito Bootstrap para simular resistores de valor elevado.

\footnotetext{
${ }^{1}$ Este principio, enunciado por el Dr. Julio Brizzi, establece que la cantidad de dificultad siempre permanece constante. Si con algo de ingenio se consigue eliminarla de un lugar, la dificultad se alojará en otro a la espera de manifestarse nuevamente.
} 


\subsection{Resistencia equivalente.}

Para calcular la resistencia equivalente, resulta útil aplicar el Teorema de Thevenin entre el punto "A" y masa (ver Fig.A4.1). Si se considera además un seguidor con ganancia exactamente unitaria, el esquema admite el siguiente circuito equivalente:

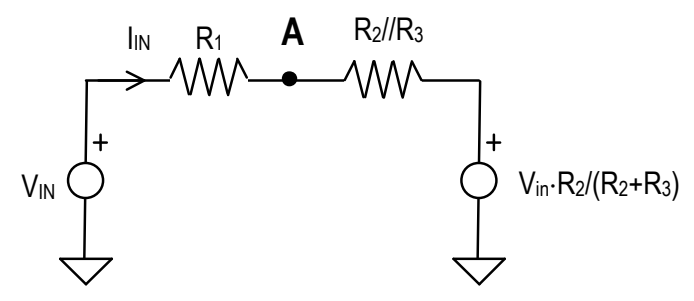

Figura A4.2. Circuito Equivalente del circuito de la Fig. A4.1.

La corriente de entrada está dada por:

y la resistencia de entrada $R_{\mathrm{EQ}}$ resulta:

$$
i_{\mathrm{IN}}=\frac{V_{\mathrm{IN}}}{R_{1}+R_{2}+R_{1} R_{2} / R_{3}},
$$

$$
R_{\mathrm{EQ}}=V_{\mathrm{IN}} / i_{\mathrm{IN}}=R_{1}+R_{2}+R_{1} R_{2} / R_{3}
$$

Como el objetivo es simular resistores de valor elevado, en general $R_{\mathrm{EQ}} \gg\left(R_{1}+R_{2}\right)$ y la ecuación (A4.2) puede aproximarse por:

$$
R_{\mathrm{EQ}} \cong R_{1} \cdot R_{2} / R_{3}
$$

Se puede interpretar, a partir de este resultado, que el circuito "amplifica" el resistor $R_{1}$ en el factor $R_{2} / R_{3}$. En general, para conseguir altas $R_{\mathrm{EQ}}$, se utiliza un resistor $R_{1}$ tan grande como sea posible y un $R_{3}$ de bajo valor.

\subsection{Apartamientos respecto de un resistor "real".}

Si bien este circuito permite simular resistores de elevado valor, el resistor equivalente no responde exactamente a las características de un resistor real de igual valor. Como se verá en esta sección, sobre sus terminales aparecerán manifestaciones de su naturaleza "activa": una tensión de offset relacionada con la correspondiente al A.O y una tensión de ruido equivalente $e_{\mathrm{n}}$ mayor que la correspondiente a la combinación de las resistencias involucradas.

Es importante notar que más allá de lo predicho por la ecuación (A4.3), la máxima impedancia a simular está acotada por la capacidad de entrada del A.O: un elemento que permanece oculto en el circuito de la Fig. A4.1. 


\subsubsection{Tensión de offset.}

El "resistor equivalente", aún sin corriente circulando sobre él, presentará una tensión de continua entre sus terminales debida a la tensión de offset $V_{\text {Os }}$ propia del amplificador operacional. Para analizar este efecto puede utilizarse el circuito equivalente de la Fig. A4.3, que modela la tensión $V_{\mathrm{OS}}$ mediante un generador de tensión de continua en la entrada del A.O.

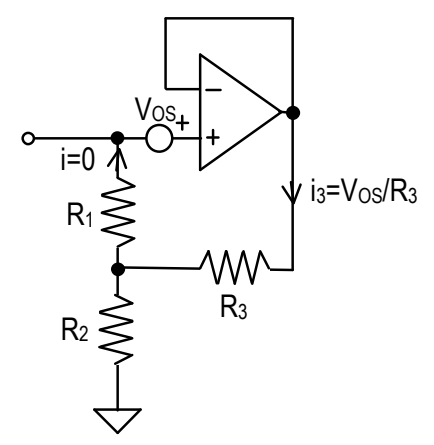

Figura A4.3. Circuito que tiene en cuenta la tensión de offset propia del A.O.

Si se considera que la entrada permanece a circuito abierto, no circulará corriente alguna sobre resistor $R_{1}$, no habrá diferencia de potencial sobre él y la tensión $V_{\mathrm{OS}}$ queda aplicada ${ }^{2}$ sobre $R_{3}$. La corriente sobre $R_{3}$ será:

$$
i_{3}=V_{\mathrm{OS}} / R_{3}
$$

y la tensión de continua $V_{\text {i.DC }}$ presente a la entrada resulta:

$$
V_{\mathrm{i} . \mathrm{DC}}=i_{3} \cdot R_{2}=V_{\mathrm{OS}} \cdot R_{2} / R_{3}
$$

Es decir que en este caso, la tensión DC sobre $R_{\mathrm{EQ}}$ es igual a la tensión de offset propia del A.O amplificada aproximadamente por el mismo factor que $R_{1}$. Este circuito amplifica resistencia y offset por el mismo factor, lo cual limita la máxima relación $R_{2} / R_{3}$ admisible, pues $V_{\text {i.DC }}$ puede llegar a ser tan alta como para comprometer el rango de entrada del amplificador operacional.

\subsubsection{Corriente de Polarización.}

Las entradas del A.O. del circuito de la Fig. A4.1 requieren corrientes de polarización $i_{\text {BIAS }}$ para operar correctamente. Estas corrientes deben tener un camino a masa, que si no es

\footnotetext{
${ }^{2}$ Se supone que el A.O tiene una ganancia de lazo abierto suficiente como para considerar una diferencia de potencial nula entre sus entradas.
} 
aportado por el generador de señal, se establecerá sobre $R_{\mathrm{EQ}}$ produciendo una tensión de DC dada por:

$$
V_{\mathrm{i} . \mathrm{DC}}=i_{\mathrm{BIAS}} \cdot R_{\mathrm{EQ}}
$$

\subsubsection{Tensión de ruido equivalente.}

Para calcular la tensión de ruido sobre el resistor equivalente, se utilizará el modelo de la Fig.A4.4. Este circuito incorpora los generadores de ruido que representan el aporte de los resistores $R_{1}, R_{2}, R_{3}$ y los correspondientes al A.O.

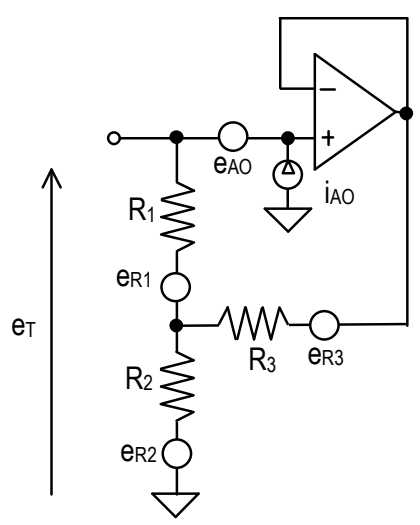

Figura A4.4. Circuito que tiene en cuenta la tensión de ruido propia del A.O.

Resolviendo este circuito, y teniendo en cuenta que los aportes de los distintos generadores deben sumarse en sus valores cuadráticos, el ruido total resulta:

$$
e_{\mathrm{T}}^{2}=e_{\mathrm{R} 1}^{2} \cdot\left(1+R_{2} / R_{3}\right)^{2}+e_{\mathrm{R} 3}^{2} \cdot\left(R_{2} / R_{3}\right)^{2}+e_{\mathrm{R} 2}^{2}+e_{\mathrm{AO}}^{2} \cdot\left(R_{2} / R_{3}\right)^{2}+i_{\mathrm{AO}}^{2} R_{\mathrm{EQ}}^{2} \cdot
$$

Reemplazando en (A4.7) la expresión de $R_{\mathrm{EQ}}$ dada por (A4.3):

$$
e_{\mathrm{T}}^{2}=e_{\mathrm{R} 1}^{2} \cdot\left(1+R_{2} / R_{3}\right)^{2}+e_{\mathrm{R} 3}^{2} \cdot\left(R_{2} / R_{3}\right)^{2}+e_{\mathrm{R} 2}^{2}+e_{\mathrm{AO}}^{2} \cdot\left(R_{2} / R_{3}\right)^{2}+i_{\mathrm{AO}}^{2} R_{1}^{2}\left(R_{2} / R_{3}\right)^{2}
$$

Dado que usualmente $R_{3} \ll R_{1}$, es decir $e_{\mathrm{R} 3} \ll e_{\mathrm{R} 1}$, es posible despreciar el segundo término frente al primero. El aporte de $R_{2}$ también puede despreciarse, porque aunque su valor es generalmente comparable a $R_{1}$, su ruido propio no es amplificado. Teniendo en cuenta estas consideraciones, el ruido total cuadrático puede aproximarse por:

$$
e_{\mathrm{T}}^{2} \cong\left(e_{\mathrm{R} 1}^{2}+e_{\mathrm{AO}}^{2}+i_{\mathrm{AO}}^{2} R_{1}^{2}\right) \cdot\left(R_{2} / R_{3}\right)^{2}
$$


y su valor RMS es:

$$
e_{\mathrm{T}} \cong \sqrt{e_{\mathrm{R} 1}^{2}+e_{\mathrm{AO}}^{2}+i_{\mathrm{AO}}^{2} R_{1}^{2}} \cdot\left(R_{2} / R_{3}\right)
$$

En general, el ruido dominante es debido a $R_{1}$ y la expresión (A4.10) puede aproximarse por:

$$
e_{\mathrm{T}} \cong e_{\mathrm{R} 1} \cdot\left(R_{2} / R_{3}\right)
$$

El ruido total también puede expresarse en función del ruido de un resistor real de $R_{\mathrm{EQ}}$ ohms como:

$$
e_{\mathrm{T}} \cong e_{\mathrm{REQ}} \cdot \sqrt{R_{2} / R_{3}}
$$

Es decir que el ruido de este resistor "simulado" es mayor que el correspondiente a un resistor real de igual valor.

\section{Un Ejemplo.}

Si se considera ruido blanco, es posible calcular el ruido en tensión RMS por unidad de raíz cuadrada de Hz. En esta condición, un resistor real de $1 \mathrm{G} \Omega$, presenta un ruido de:

$$
e_{\mathrm{R}=1 \mathrm{G} \Omega}=\sqrt{4 k T R}=4 \mu \mathrm{V} / \sqrt{\mathrm{Hz}}
$$

y un resistor "simulado" de $1 \mathrm{G} \Omega$, implementado con $R_{1}=10 \mathrm{M} \Omega, R_{2} / R_{3}=100$, un A.O con un ruido propio $e_{\mathrm{AO}}=10 \mathrm{nV} / \sqrt{\mathrm{Hz}}$ y $i_{\mathrm{AO}}=1 \mathrm{fA} / \sqrt{\mathrm{Hz}}$, presentará para $T=300 \mathrm{~K}$ un ruido total de:

$$
\begin{aligned}
& e_{\mathrm{T}} \cong \sqrt{e_{\mathrm{R} 1}^{2}+e_{\mathrm{AO}}^{2}+i_{\mathrm{AO}}^{2} R_{1}^{2}} \cdot\left(R_{2} / R_{3}\right) \\
& e_{\mathrm{T}} \cong \sqrt{(400 \mathrm{nV} / \sqrt{\mathrm{Hz}})^{2}+(10 \mathrm{nV} / \sqrt{\mathrm{Hz}})^{2}+(10 \mathrm{nV} / \sqrt{\mathrm{Hz}})^{2}} \cdot 100 \\
& e_{\mathrm{T}} \cong 40 \mu \mathrm{V} / \sqrt{\mathrm{Hz}}
\end{aligned}
$$

Es decir unas 10 veces mayor que un resistor real. También se observa en esta ecuación, que el aporte del A.O. es despreciable frente al de $R_{1}$.

\subsection{Modelo Equivalente.}

Finalmente, componiendo lo desarrollado en los puntos previos, se concluye que el modelo del resistor equivalente contiene, además del resistor mismo, un generador de tensión de DC, un generador de corriente de DC y un generador de ruido cuyo valor es mayor que el correspondiente al ruido térmico de un resistor real de igual valor. Todo esto se resume en el circuito de la Fig.A4.5. 


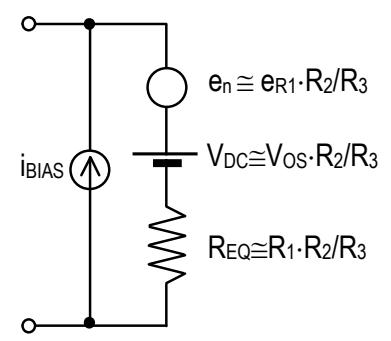

Figura A4.5. Modelo equivalente del resistor simulado.

\section{Una aplicación. El Amplificador Bootstrap Clásico.}

Cuando la señal de entrada de un I.A. es acoplada capacitivamente, se requiere incluir un resistor conectado a masa para proveer un camino de DC a las corrientes de polarización del amplificador. La degradación de la impedancia de entrada que este resistor introduce puede minimizarse utilizando la técnica descripta anteriormente. El circuito resultante, conocido como "amplificador bootstrap" se presenta en la Fig.A4.6.a.

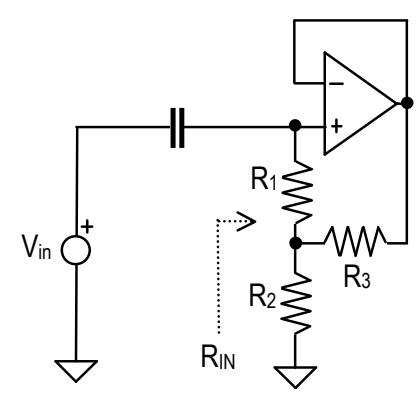

(a)

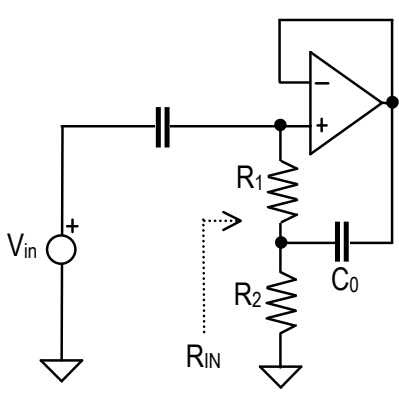

(b)

Figura A4.6. (a) Técnica de bootstraping para conseguir elevadas impedancias de entrada. (b) Circuito alternativo que permite evitar la amplificación de la tensión de offset del A.O.

Para evitar la amplificación de la tensión de offset del A.O. analizada en el punto 2.2.1, el resistor $R_{3}$ debe reemplazarse por un capacitor $C_{0}$ (PALLAS-ARENY, 1986), resultando el circuito de la Fig.A4.1.b. En este caso, la realimentación positiva (bootstraping) funcionará solo en CA y la nueva impedancia de entrada resulta:

$$
R_{\mathrm{IN}}=R_{1}+R_{2}+R_{1} R_{2} C_{0} \cdot \mathrm{s}
$$


Esta solución es apropiada para aplicaciones biomédicas (PALLAS-ARENY, 1986) (PALLAS-ARENY, 1989.a), pues no se requiere una muy alta impedancia de entrada en DC sino para $50 \mathrm{~Hz}$. A la frecuencia de red, eligiendo adecuadamente los valores de $R_{1}, R_{2}$ y $C_{0}$, sí es posible conseguir una elevada impedancia de entrada. Este circuito presenta una ganancia unitaria pero provee acoplamiento en AC. El mismo ha sido utilizado en aplicaciones biomédicas combinado con un amplificador de instrumentación (Fig.A4.7.b) que aporta la necesaria amplificación diferencial.

El hecho que la etapa de entrada esté compuesta por dos circuitos bootstrap totalmente independientes entre sí (Fig.A4.7.a), conduce a bajos rechazos de modo común debido a los desbalances que pudieran existir entre ellos (PALLAS-ARENY, 1991.d). Para solucionar esto, (RAMOS, 1999) propone acoplar los circuitos de entrada través de un resistor $R_{\mathrm{C}}$ compartido, resultando el circuito de la Fig.A4.7.b. De este modo, una variación en el valor de este resistor afectará a ambas ramas por igual colaborando a mantenerlas balanceadas.

El CMRR de este último circuito depende de $R_{\mathrm{C}}$ y es mayor cuanto más elevado sea el valor de este resistor. En lugar de un resistor $R_{\mathrm{C}}$ "real" puede utilizarse, un segundo circuito bootstrap como el de la Fig. A4.1.a.

Con este circuito pueden obtenerse CMRR de más de $90 \mathrm{~dB}$, lo cual es suficiente para muchas aplicaciones biomédicas. Su principal inconveniente es que no aporta ganancia mientras que su ruido propio es amplificado por las etapas subsiguientes Esta solución no es apropiada para aplicaciones que requieran un muy bajo nivel de ruido como EEG o ECG de alta resolución.

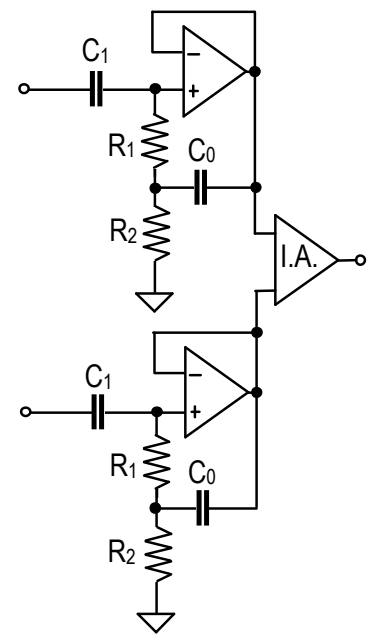

(a)

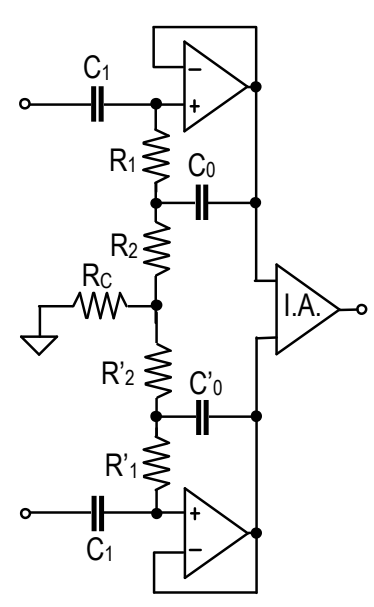

(b)

Figura A4.7. (a) Amplificador para Biopotenciales utilizando dos buffers independientes.

(b) Circuito que mejora el CMRR acoplando los buffers a través de $R_{c}$. 
Apéndice 4 - Simulación de Altas Resistencias 


\section{Apéndice 5 \\ CMRR en Redes Diferenciales.}

\section{CMRR Infinito.}

Existen al menos dos formas para que una red con entrada y salida diferencial (F-D: Fully-Differential) presente un CMRR infnito aún en presencia de desbalances entre sus componentes.

1. Una red perfectamente flotante sin conexión alguna a masa.

2. Un circuito conectado a masa mediante generadores de tensión iguales a la tensión de modo común de entrada.

La opción 1 es simple de cumplir en circuitos pasivos, pero si se incorporan elementos activos puede resultar de difícil implementación. Por ejemplo, la salida de un A.O es un generador de tensión conectado a masa; siendo además necesarios caminos a masa para las corrientes de polarización de entrada de los amplificadores. En estos casos la opción 2 es más plausible.

\section{Redes Flotantes.}

\subsection{Enunciado.}

"Dado un circuito cualquiera con entrada y salida diferencial, si éste no contiene ninguna impedancia conectada a masa, entonces su CMRR será infinito."

\subsection{Demostración.}

En la Fig.A5.1 se muestra la condición descripta en el enunciado. Si se aplica a la entrada de la red una tensión de modo común $V_{\mathrm{iC}}$, al no existir ninguna conexión entre esta red y masa, no circulará corriente alguna en este circuito (no es posible establecer ninguna malla cerrada que contenga al generador $V_{\mathrm{iC}}$ ), y por lo tanto todos sus nodos adoptarán el potencial ${ }^{1} V_{\mathrm{ic}}$. Cualquier tensión de modo diferencial definida sobre esta red será nula, por ejemplo la tensión diferencial de salida.

Dado que al aplicar una tensión de entrada de modo común la salida diferencial del circuito es nula, su CMRR resulta infinito.

Es importante notar que el CMRR de la red será infinito independientemente de los desbalances que pudieran existir entre sus componentes. El circuito podría inclusive no ser simétrico.

\footnotetext{
${ }^{1}$ Dicho de un modo más simple: imaginemos una compleja red enmarañada que toca solo el borne positivo de una batería; al no existir conexión con el borne negativo no hay manera que circule corriente alguna y todos los nodos del circuito estarán al potencial del terminal positivo.
} 


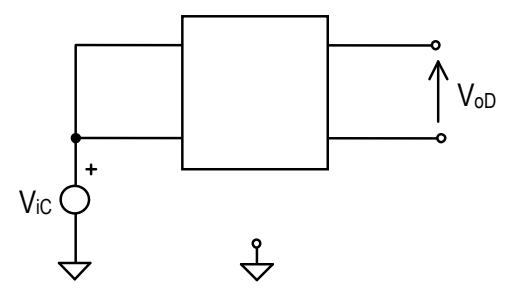

Figura A5.1. Circuito Diferencial flotante con una entrada de modo común $V_{i c .}$

\section{Redes con conexión a masa.}

\subsection{Enunciado.}

"Dado un circuito con entrada y salida diferenciales, si las únicas conexiones a masa que éste presenta son realizadas a través de generadores de tensión, cuyos valores coinciden con la tensión de modo común de entrada $V_{\mathrm{iC}}$, su CMRR será infinito independientemente de los desbalances entre sus componentes".

\subsection{Demostración.}

Si tanto la tensión de entrada como todas las vinculaciones de la red con masa son realizadas a través de generados de tensión $V_{\mathrm{iC}}$, todas la mallas cerradas que puedan definirse no contendrán ningún generador de tensión o sino dos generadores iguales en oposición, es decir que la tensión neta de los generadores de todas las mallas posibles serán nulas (ver Fig.A5.2). Como consecuencia de esto, todas las corrientes de malla serán nulas así también como todas las corrientes del circuito; es decir que se repiten las mismas condiciones que en el punto 2: todos los nodos se encontrarán al potencial $V_{\text {ic }}$, la tensión diferencial de salida de la red será nula y su CMRR infinito.

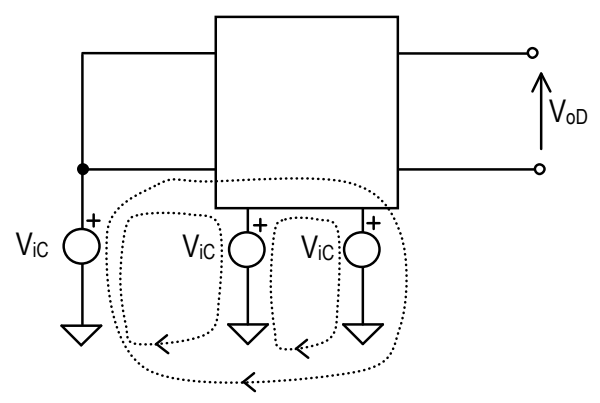

Figura A5.2. Circuito F-D conectado a masa mediante generadores de tensión iguales a la tensión de modo común de entrada $\mathrm{V}_{\mathrm{ic}}$. 


\section{Apéndice 6}

\section{Cálculo del CMRR y Ruido Propio para el I.A. Fully-Differential de dos A.O.}

\section{Cálculo del CMRR.}

Si se consideran A.O. ideales, el CMRR de este circuito resulta infinito e independiente del desbalance que pudiera existir entre los resistores. En la práctica, este rechazo se encuentra limitado por el CMRR y por la ganancia de lazo abierto propia de los A.O. que lo componen. Incluyendo estos efectos (ver APÉNDICE 7) el circuito equivalente resulta:

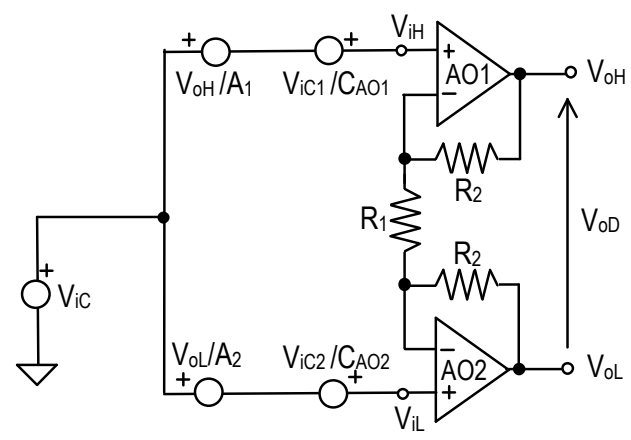

Figura A6.1. Circuito Equivalente del I.A. de dos A.O. que contempla el CMRR y la ganancia de lazo abierto de los A.O.

Si se aplica una entrada de modo común $V_{\mathrm{iC}}$, la tensión diferencial de salida $V_{\mathrm{oD}}$ resulta:

$$
V_{\mathrm{oD}}=V_{\mathrm{oH}}-V_{\mathrm{oL}}=\left(-\frac{V_{\mathrm{iCl}}}{C_{\mathrm{AO} 1}}+\frac{V_{\mathrm{iC} 2}}{C_{\mathrm{AO} 2}}+\frac{V_{\mathrm{oH}}}{A_{1}}-\frac{V_{\mathrm{oL}}}{A_{2}}\right) \cdot G_{\mathrm{DD}} \cdot
$$

La tensión de modo común de entrada de ambos A.O. coincide con la tensión de entrada, esto es:

$$
V_{\mathrm{iC} 1} \cong V_{\mathrm{iC} 2} \cong V_{\mathrm{iC}}
$$

Por otra parte, como este circuito presenta una ganancia de modo común $G_{\mathrm{CC}}$ unitaria, es posible escribir:

$$
\begin{aligned}
& V_{\mathrm{oH}} \cong V_{\mathrm{iC}}+V_{o D} / 2 \\
& V_{\mathrm{oL}} \cong V_{\mathrm{iC}}-V_{o D} / 2
\end{aligned}
$$

Utilizando las aproximaciones (A6.2) y (A6.3) en (A6.1), resulta:

$$
V_{\mathrm{oD}}=V_{\mathrm{iC}}\left(\frac{1}{C_{\mathrm{AO} 2}}-\frac{1}{C_{\mathrm{AO} 1}}\right) \cdot G_{\mathrm{DD}}+\left(\frac{V_{\mathrm{iC}}-V_{\mathrm{oD}} / 2}{A_{1}}-\frac{V_{\mathrm{iC}}+V_{\mathrm{oD}} / 2}{A_{2}}\right) \cdot G_{\mathrm{DD}},
$$


y agrupando términos:

$$
V_{\mathrm{oD}}\left\{1+\frac{G_{\mathrm{DD}}}{2 A_{1}}+\frac{G_{\mathrm{DD}}}{2 A_{2}}\right\}=V_{\mathrm{iC}} \cdot\left(1 / C_{\mathrm{AO} 2}-1 / C_{\mathrm{AO} 1}+1 / A_{1}-1 / A_{2}\right) \cdot G_{\mathrm{DD}} \cdot
$$

Considerando, que al menos en el rango de frecuencias de trabajo, $G_{\mathrm{DD}} \ll A_{1}, A_{2}$, la ganancia $\mathrm{G}_{\mathrm{DC}}$ queda dada aproximadamente por:

$$
G_{D C}=\frac{V_{o D}}{V_{i C}} \cong\left\{\left(1 / C_{A O 2}-1 / C_{A O 1}\right)+\left(1 / A_{1}-1 / A_{2}\right)\right\} \cdot G_{D D}
$$

y el $C M R R$ resulta:

$$
\operatorname{CMRR}^{-1}=\left(C_{\mathrm{AO} 2}^{-1}-C_{\mathrm{AO} 1}^{-1}\right)+\left(A_{1}^{-1}-A_{2}^{-1}\right) \quad .
$$

Esta última expresión pone en evidencia la principal ventaja de esta configuración: su CMRR no depende del desapareamiento entre los resistores sino exclusivamente de los desbalances en el CMRR y en la ganancia de lazo abierto de los A.O.

En general, el primer término en (A6.7) es dominante, dado que las ganancias $A_{1}$ y $A_{2}$ son de igual signo y de similar valor, mientras que los rechazos individuales $C_{\mathrm{AO} 1} \mathrm{y}$ $C_{\mathrm{AO} 2}$ pueden diferir significativamente tanto en magnitud como en signo ${ }^{1}$. Teniendo esto en cuenta, la ganancia $G_{\mathrm{DC}}$ y el CMRR de este circuito pueden aproximarse por:

$$
\begin{aligned}
& G_{\mathrm{DC}}=\left(1 / C_{\mathrm{AO} 2}-1 / C_{\mathrm{AO} 1}\right) \cdot G_{\mathrm{DD}} \\
& C M R R^{-1}=C_{\mathrm{AO} 2}^{-1}-C_{\mathrm{AO} 1}^{-1}
\end{aligned}
$$

\section{Tensión de Ruido Equivalente a la Entrada.}

Para estimar el ruido total referido a la entrada del amplificador, es necesario considerar en su circuito equivalente los generadores de ruido propios de los A.O. de entrada y los asociados a los resistores. Esto conduce al circuito de la Fig.A6.2. donde no se incluyeron generadores de corriente de ruido porque se suponen A.O de tecnología FET o CMOS cuyas corrientes de ruido son despreciables.

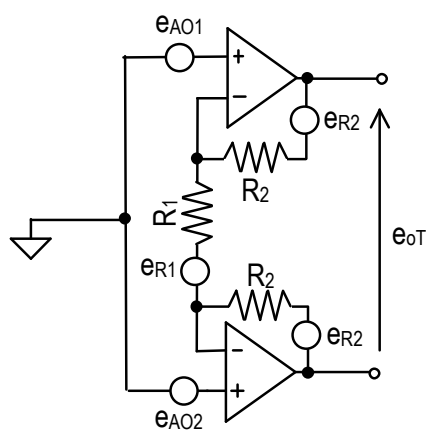

Figura A6.2. Circuito Equivalente del I.A. de dos A.O. que contempla el ruido propio de los A.O y de los resistores.

\footnotetext{
${ }^{1}$ Se considera aquí un modelo de bajas frecuencias donde $A_{1}, A_{2}, C_{\mathrm{AO} 1}$ y $C_{\mathrm{AO} 2}$ son escalares. En un modelo de mayor alcance todas estas magnitudes son complejas.
} 
A partir del circuito de la Fig.A6.2 se encuentra que la tensión media cuadrática de ruido a la salida, está dada por:

$$
e_{\mathrm{oT}}^{2}=\left(e_{\mathrm{AO} 1}^{2}+e_{\mathrm{AO} 2}^{2}+e_{\mathrm{R} 1}^{2}\right) \cdot G_{\mathrm{DD}}^{2}+2 \cdot e_{\mathrm{R} 2}^{2}
$$

Si se consideran resistores con bajo exceso de ruido, es decir con un ruido propio igual al teórico dado por:

$$
e_{R 2}^{2}=4 K T B R_{2},
$$

y utilizando que $R_{2}$ y $R_{1}$ están relacionados a través de la ganancia $G_{\mathrm{DD}}$ según:

$$
R_{2}=\left(G_{\mathrm{DD}}-1\right) / 2 \cdot R_{1} \cong G_{\mathrm{DD}} R_{1} / 2,
$$

el ruido de $R_{2}$ puede expresarse en función del ruido de $R_{1}$ como:

$$
e_{\mathrm{R} 2}^{2}=e_{\mathrm{R} 1}^{2} \cdot G_{\mathrm{DD}} / 2
$$

Reemplazando esta última expresión en (A6.10), resulta:

$$
e_{\mathrm{oT}}^{2}=\left(e_{\mathrm{AO} 1}^{2}+e_{\mathrm{AO} 2}^{2}\right) \cdot G_{\mathrm{DD}}^{2}+e_{\mathrm{R} 1}^{2} \cdot\left(G_{\mathrm{DD}}^{2}+G_{\mathrm{DD}}\right) \text {. }
$$

Generalmente el objetivo del I.A. es amplificar $\left(G_{\mathrm{DD}}\right.$ » 1$)$ y el ruido total referido a la entrada $e_{\mathrm{iT}}$, puede aproximarse por:

$$
e_{\mathrm{iT}}^{2} \cong e_{\mathrm{AO} 1}^{2}+e_{\mathrm{AO} 2}^{2}+e_{\mathrm{R} 1}^{2}
$$

Si se tiene cuidado en la elección de $R_{1}$, su contribución no será importante y el ruido total corresponderá a los dos A.O. que componen la etapa de entrada, es decir:

$$
e_{\mathrm{iT}}^{2} \cong 2 \cdot e_{\mathrm{AO} 1}^{2}
$$


Apéndice 6 - Cálculo del CMRR y ruido Propio para el I.A. Fully-Differential de dos A.O. 


\section{Apéndice 7.}

\section{Modelización de la Ganancia de Lazo Abierto y CMRR de un A.O.}

\section{Ganancia Finita.}

Si se considera que el A.O. tiene una ganancia infinita, su tensión diferencial de entrada será nula para cualquier valor finito de su salida. Entonces, entre sus bornes de entrada, no existirá diferencia de potencial alguna. Esta última condición resulta muy útil para resolver en forma simple, circuitos que involucren amplificadores operacionales.

Si se acepta ahora que el A.O. tiene una ganancia finita $A$, su tensión de entrada no será nula, sino que para producir la tensión $V_{\mathrm{o}}$ presente en su salida, deberá existir una tensión de entrada $\varepsilon=V_{\mathrm{o}} / A$. Esta situación, también podría describirse mediante con un A.O. ideal (de ganancia infinita, es decir con entrada nula) y un generador de tensión de valor $V_{\mathrm{o}} / A$ tal como se muestra en la Fig.A7.1. Como el A.O. de este nuevo circuito es ideal, para resolver los circuitos puede nuevamente considerarse que sus entradas se encuentran a igual potencial.

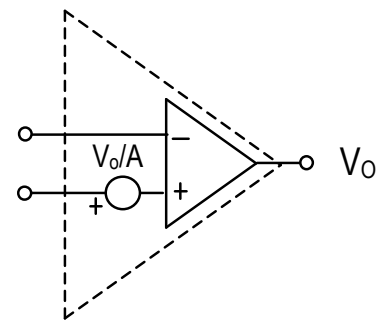

Figura. A7.1. Circuito equivalente de un A.O con ganancia finita. El nuevo modelo está compuesto por un A.O. ideal y un generador de tensión dependiente de la tensión de salida.

\section{Rechazo de Modo Común.}

Si a un A.O. real se le aplica una tensión de modo común $V_{\mathrm{iC}}$, su salida no será nula sino que va a estar dada por su rechazo de modo común $C_{\mathrm{AO}}$ según:

$$
V_{\mathrm{o}}=V_{\mathrm{iC}} \cdot \frac{A}{C_{\mathrm{AO}}}
$$

siendo $A$ la ganancia del A.O. Esto también podría describirse considerando un A.O. de CMRR infinito, pero con una tensión diferencial neta en su entrada dada por un generador de tensión externo $V_{\mathrm{iC}} / C_{\mathrm{AO}}$, tal como se muestra en la Fig.A7.2. 


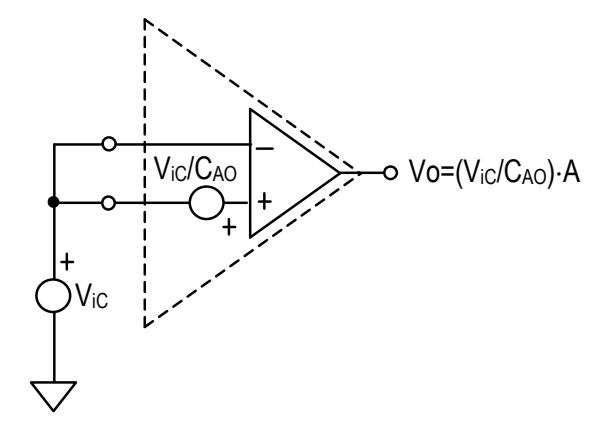

Figura. A7.2. Circuito equivalente de un A.O con CMRR finito. El nuevo modelo está compuesto por un A.O. ideal y un generador de tensión dependiente de la tensión de modo común de entrada $\bigvee_{i c}$.

Utilizando esta técnica, un circuito compuesto por A.O. con CMRR finito, puede llevarse a otro equivalente compuesto por A.O. ideales. Este nuevo circuito contiene generadores de tensión dependientes de la tensión de modo común $V_{\mathrm{iC}}$ presente en las entradas de los A.O. 


\section{Apéndice 8}

\section{Cálculo del CMRR para el Amplificador de Diferencia.}

\section{Amplificador de Diferencia.}

Esta topología presenta una baja impedancia de entrada (CHRISTIANSEN, 1982) y por tal motivo no es adecuada para amplificadores de biopotenciales. Igualmente se analiza aquí, porque forma parte de estructuras multietapas como el clásico I.A. de tres A.O.

El circuito equivalente, considerando el CMRR propio del A.O. (APÉNDICE 7), se muestra en la Fig.A8.1. .

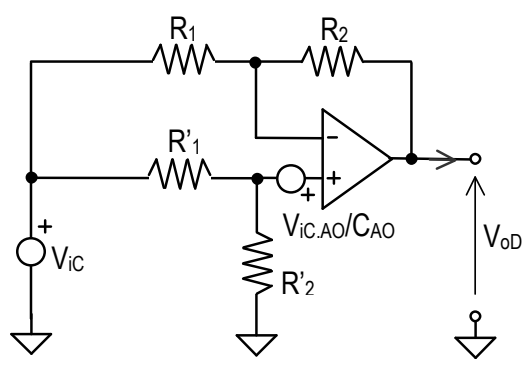

Figura A8.1. Circuito Equivalente del Amplificador de Diferencia considerando el CMRR propio del A.O.

Para determinar la ganancia CMRR de este circuito, en primer lugar se calculará su ganancia cruzada $G_{\mathrm{DC}}$. Si se aplica a la entrada una tensión de modo común $V_{\mathrm{iC}}$, la tensión diferencial ${ }^{1}$ de salida estará dada por:

$$
V_{\mathrm{oD}}=V_{\mathrm{iC}} \cdot G_{\mathrm{DC}}
$$

Resolviendo el circuito para esta situación, se obtiene.

$$
V_{\mathrm{oD}}=V_{\mathrm{iC}} \cdot\left\{\frac{R_{2}^{\prime}}{R_{1}^{\prime}+R_{3}^{\prime}} \cdot\left(1+\frac{R_{2}}{R_{1}}\right)-\frac{R_{2}}{R_{1}}\right\}+\frac{V_{\mathrm{iC} . \mathrm{AO}}}{C_{\mathrm{AO}}} \cdot\left\{1+\frac{R_{2}}{R_{1}}\right\} .
$$

La tensión de modo común $V_{\text {ic.AO }}$, presente en la entrada del A.O, puede expresarse a partir de la tensión de modo común de entrada $V_{\text {iC }}$ según:

$$
V_{\mathrm{iC} . \mathrm{AO}}=V_{\mathrm{iC}} \cdot \frac{R_{2}^{\prime}}{R_{1}^{\prime}+R_{2}^{\prime}}
$$

\footnotetext{
${ }^{1}$ En este caso, la salida del circuito es de tipo "single ended" (S-E): uno de los bornes de salida se encuentra conectado a masa (ver Fig.A8.1).
} 
Reemplazando (A8.3) en (A8.2) y luego de un poco de álgebra, (A8.2) puede escribirse como:

$$
V_{\mathrm{oD}}=V_{\mathrm{iC}} \cdot \frac{R_{2}}{R_{1}}\left\{\frac{1+R_{1} / R_{2}}{1+R_{1}^{\prime} / R_{2}^{\prime}}-1\right\}+\frac{V_{\mathrm{iC}}}{C_{\mathrm{AO}}} \cdot \frac{R_{2}}{R_{1}} \frac{1+R_{1} / R_{2}}{1+R_{1}^{\prime} / R_{2}^{\prime}} .
$$

Llamando

$$
\alpha=\frac{1+R_{1} / R_{2}}{1+R_{1}^{\prime} / R_{2}^{\prime}}-1=\frac{R_{1} / R_{2}-R_{1}^{\prime} / R_{2}^{\prime}}{1+R_{1}^{\prime} / R_{2}^{\prime}} \quad,
$$

y teniendo en cuenta la ganancia diferencial $G_{\mathrm{DD}}$ de este circuito esta dada aproximadamente ${ }^{2}$ por:

$$
G_{\mathrm{DD}} \cong \frac{R_{2}}{R_{1}}
$$

la ecuación (A8.4) se transforma en:

$$
V_{\mathrm{oD}}=V_{\mathrm{iC}} \cdot G_{\mathrm{DD}} \cdot\left\{\alpha+\frac{(\alpha+1)}{C_{\mathrm{AO}}}\right\} .
$$

El parámetro $\alpha$ depende exclusivamente de los resistores externos y más precisamente del desbalance en las relaciones $R_{2} / R_{1}$ y $R_{2}{ }_{2} / R_{1}{ }_{1}$. En un amplificador diferencial razonablemente construido $\alpha$ será mucho menor que $1 \mathrm{y}$ la ecuación (A8.7) puede aproximarse por:

$$
G_{\mathrm{DC}}=\left\{\alpha+\frac{1}{C_{\mathrm{AO}}}\right\} \cdot G_{\mathrm{DD}}
$$

Es posible expresar $\alpha$ en función de la tolerancia relativa $t=\Delta R / R$ de los resistores utilizados. El peor caso, es decir el mayor valor de $\alpha$, se produce cuando las tolerancias confabulan haciendo:

$$
R_{1}=R_{10}(1+t) ; R_{2}=R_{20}(1-t) ; R_{1}=R_{10}^{\prime}(1-t) ; R_{20}^{\prime}(1+t)
$$

siendo $R_{10}, R_{20}, R^{\prime}{ }_{10}$ y $R^{\prime}{ }_{20}$ los valores nominales de los resistores y $t$ su tolerancia. En este caso resulta:

$$
\alpha=\frac{\frac{R_{10}(1+t)}{R_{20}(1-t)}-\frac{R_{10}(1-t)}{R_{20}(1+t)}}{1+\frac{R_{10}(1-t)}{R_{20}(1+t)}} \cong \frac{\frac{R_{10}(1+t)}{R_{20}(1-t)}-\frac{R_{10}(1-t)}{R_{20}(1+t)}}{1+\frac{R_{10}}{R_{20}}},
$$

que puede aproximarse por:

$$
\alpha \cong \frac{\frac{(1+t)}{(1-t)}-\frac{(1-t)}{(1+t)}}{1+\frac{R_{20}}{R_{10}}}=\frac{\frac{4 \cdot t}{1-(t)^{2}}}{1+\frac{R_{20}}{R_{10}}} .
$$

\footnotetext{
${ }^{2}$ Esto implica considerar bajas frecuencias y desbalances razonables entre los resistores.
} 
Considerando tolerancias razonables la expresión (A8.11) puede escribirse como:

$$
\alpha \cong \frac{4 \cdot t}{1+\frac{R_{20}}{R_{10}}}
$$

Reemplazando (A8.12) en (A8.8) y recordando que la ganancia diferencial está dada aproximadamente por $G_{\mathrm{DD}} \cong R_{2} / R_{1}$, finalmente puede expresarse $G_{\mathrm{DC}}$ como:

$$
G_{\mathrm{DC}}=\frac{4 \cdot t}{1+1 / G_{\mathrm{DD}}}+\frac{G_{\mathrm{DD}}}{C_{\mathrm{AO}}}
$$

El primer término depende de la tolerancia de los resistores y el segundo del CMRR propio del A.O. Para que estos términos sean comparables, la tolerancia $t$ de los resistores debería cumplir:

$$
t \approx \frac{1+1 / G_{\mathrm{DD}}}{4 C_{\mathrm{AO}}}
$$

Por ejemplo, considerando un A.O. con un CMRR bajo, de unos $80 \mathrm{~dB}$ (10.000 veces) y aún adoptando una baja $G_{\mathrm{DD}}$, por ejemplo unitaria ${ }^{3}$, resultan tolerancias de $0.005 \%$. Esto no es simple de conseguir y en general $G_{\mathrm{DC}}$ estará dominada por la tolerancia de los resistores y será aproximadamente:

$$
G_{\mathrm{DC}} \cong \frac{4 \cdot t}{1+1 / G_{\mathrm{DD}}} .
$$

Finalmente, el Rechazo de Modo Común para esta configuración resulta:

$$
C M R R=\frac{G_{\mathrm{DD}}}{G_{\mathrm{DC}}} \cong \frac{1+G_{\mathrm{DD}}}{4 \cdot t}
$$

Una práctica usual para mejorar el CMRR de esta configuración, es incorporar un "ajuste funcional". Mediante un elemento variable (usualmente el resistor $R_{2}$ ), se ajusta $^{4}$ su valor para obtener máximo CMRR.

\footnotetext{
${ }^{3}$ Si la $G_{\mathrm{DD}}$ fuera menor que la unidad, el amplificador no sería muy útil, al menos para amplificar.

${ }^{4}$ Admitir la necesidad de un ajuste es admitir una derrota de diseño.
} 
Apéndice 8 - Cálculo del CMRR para el Amplificador de Diferencia 


\section{Apéndice 9.}

\section{Cálculo de CMRR y Ruido Propio para el Amplificador de Instrumentación de dos A.O.}

\section{Cálculo del CMRR.}

Existen diversos factores que degradan el CMRR del Amplificador de Instrumentación de dos A.O., como los desbalances entre los resistores y el CMRR propio de los amplificadores operacionales que componen el amplificador.

\subsection{Efecto del CMRR propio de los A.O.}

En la Fig.A9.1 se presenta un circuito equivalente de este I.A. que incluye el efecto del CMRR propio de los A.O.

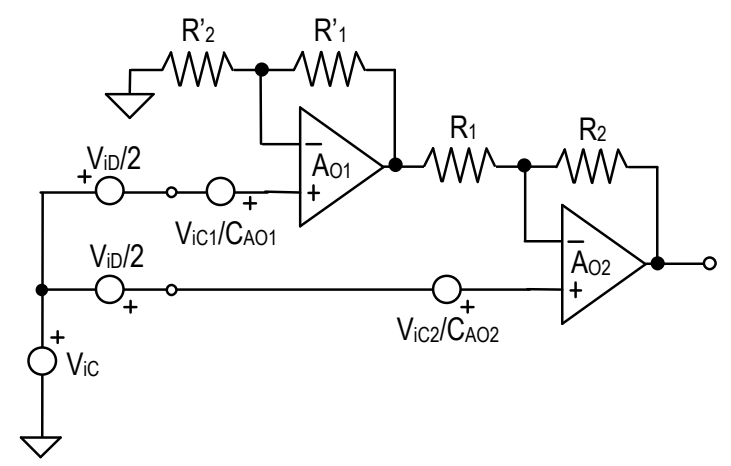

Figura A9.1. Circuito Equivalente del Amplificador de Instrumentación de dos A.O. que contempla el CMRR propio de los A.O.

La tensión de salida $V_{\mathrm{oD}}$, está dada por:

$$
\begin{aligned}
& V_{\mathrm{oD}}=\frac{V_{\mathrm{iD}}}{2} \cdot\left\{\left(1+R_{2} / R_{1}\right)+\left(1+R_{1}^{\prime} / R_{2}^{\prime}\right) \cdot R_{2} / R_{1}\right\}+\frac{V_{\mathrm{iC} 2}}{C_{\mathrm{AO} 2}} \cdot\left(1+R_{2} / R_{1}\right)- \\
& -\frac{V_{\mathrm{iC} 1}}{C_{\mathrm{AO} 1}} \cdot\left(1+R_{1}^{\prime} / R_{2}^{\prime}\right) \cdot R_{2} / R_{1}+V_{\mathrm{iC}} \cdot\left\{\left(1+R_{2} / R_{1}\right)-\left(1+R_{1}^{\prime} / R_{2}^{\prime}\right) \cdot R_{2} / R_{1}\right\}
\end{aligned}
$$

Considerando que las tensiones de modo común de entrada $V_{\mathrm{iC} 1}, V_{\mathrm{iC} 2}$ de cada uno de los A.O son aproximadamente iguales a la tensión de modo común de entrada $V_{\mathrm{iC}}$, la expresión (A9.1) puede rescribirse como:

$$
V_{\mathrm{oD}} \cong V_{\mathrm{iD}} \cdot G_{\mathrm{DD}}+V_{\mathrm{iC}} \cdot\left[\delta+\left(C_{\mathrm{AO} 1}^{-1}-C_{\mathrm{AO} 2}^{-1}\right) \cdot G_{\mathrm{DD}}\right]
$$

siendo

$$
G_{\mathrm{DD}} \cong 1+R_{2} / R_{1} \quad ; \quad \delta=1-\frac{R_{1}^{\prime} \cdot R_{2}}{R_{2}^{\prime} \cdot R_{1}}
$$


Finalmente, para frecuencias bajas, las ganancias $G_{\mathrm{DD}}$ y $G_{\mathrm{DC}}$ resultan:

$$
G_{\mathrm{DD}} \cong 1+R_{2} / R_{1} \quad ; \quad G_{\mathrm{DC}}=\delta+\left(C_{\mathrm{AO} 1}^{-1}-C_{\mathrm{AO} 2}^{-1}\right) \cdot G_{\mathrm{DD}}
$$

y el rechazo de modo común:

$$
\frac{1}{C M R R}=\frac{\delta}{G_{\mathrm{DD}}}+\left(\frac{1}{C_{\mathrm{AO} 1}}-\frac{1}{C_{\mathrm{AO} 2}}\right)
$$

Normalmente los rechazos de modo común propios de los A.O son elevados y si están además medianamente apareados, el segundo término en (A9.5) será despreciable frente al primero. En estas condiciones, el CMRR queda determinado principalmente por las tolerancias en los resistores ${ }^{1}$ y es posible aproximar (A9.5) por:

$$
\frac{1}{C M R R} \cong \frac{\delta}{G_{\mathrm{DD}}}
$$

Suponiendo resistores con igual tolerancia relativa $t=\Delta R / R$, el mayor valor de $\delta$ (peor caso) estará dado por:

$$
\delta_{\mathrm{MAX}}=1-\frac{(1+t)^{2}}{(1-t)^{2}}
$$

que para bajas tolerancias $(t \ll 1)$, puede aproximarse por:

$$
\delta_{\mathrm{MAX}} \cong 1-(1+4 \cdot t) \cong 4 \cdot t
$$

obteniendo finalmente:

$$
\frac{1}{C M R R}=\frac{4 \cdot t}{G_{\mathrm{DD}}}+\left(\frac{1}{C_{\mathrm{AO} 1}}-\frac{1}{C_{\mathrm{AO} 2}}\right) \cong \frac{4 \cdot t}{G_{\mathrm{DD}}}
$$

Esta expresión es similar a la correspondiente del amplificador de diferencia. Para conseguir CMRR elevados es necesaria un ganancia $G_{\mathrm{DD}}$ importante. Si se consigue asignar una $G_{\mathrm{DD}}$ muy elevada, se pondrá en evidencia otro problema de este amplificador: los dos A.O trabajan en condiciones muy disímiles, uno de ellos tiene una fuerte realimentación mientras que el otro una muy débil. Esto conduce a una degradación del CMRR que se agrava aún más al incrementarse la frecuencia y volverse más evidentes los efectos de la ganancia finita de los A.O. De todos modos, si la frecuencia de trabajo no es muy alta y no se requiere un elevado CMRR, es una configuración útil y utilizada (MEETING VAN RIJN, 1994)

\footnotetext{
${ }^{1}$ Es decir por el valor de $\delta$, el cual depende exclusivamente del desbalance entre los resistores.
} 


\subsection{Efecto de la Ganancia Finita de los A.O.}

Como se mencionó anteriormente, los dos A.O. que componen este amplificador de instrumentación trabajan en condiciones muy disímiles con relación a su ganancia de lazo cerrado. Debido a esto, si los A.O no tienen muy altas ganancias de lazo abierto, resulta un bajo CMRR. Para analizar este efecto, se considerará un I.A con un balance perfecto de componentes, pero compuesto por amplificadores con ganancias de lazo abierto finitas $A_{1}$ y $A_{2}$. El circuito equivalente para esta condición (APÉNDICE 7), se muestra en la Fig.A9.2.

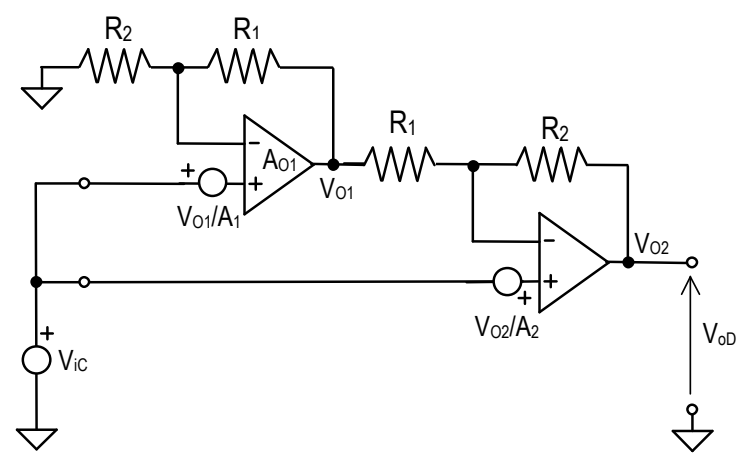

Figura A9.2. Circuito Equivalente del Amplificador de Instrumentación de dos A.O. que contempla la ganancia de lazo abierto de los A.O.

Para determinar la ganancia $G_{\mathrm{DC}}$ se considerará en ambas entradas una tensión de modo común $V_{\text {ic. }}$ A partir del circuito de la Fig.A9.2 se obtiene:

$$
\begin{gathered}
V_{\mathrm{oD}}=\left(-V_{\mathrm{o} 2} / A_{2}+V_{\mathrm{o} 1} / A_{2}\right) \cdot\left(1+R_{2} / R_{1}\right) \\
V_{\mathrm{o} 1} \cong V_{\mathrm{iC}} \cdot\left(1+R_{1} / R_{2}\right) \\
V_{\mathrm{o} 2}=V_{\mathrm{oD}} .
\end{gathered}
$$

Utilizando,

$$
1+R_{1} / R_{2}=\frac{G_{\mathrm{DD}}}{G_{\mathrm{DD}}-1}
$$

y reemplazando (A9.11) y (A9.12) en (A9.10), resulta:

$$
V_{\mathrm{oD}}=V_{\mathrm{iC}} \frac{G_{\mathrm{DD}}^{2}}{\left(1+G_{\mathrm{DD}} / A_{2}\right) \cdot A_{1} \cdot\left(G_{\mathrm{DD}}-1\right)}
$$

A partir de (A9.14) se obtiene $G_{\mathrm{DC}}$, que queda dada por:

$$
G_{\mathrm{DC}}=\frac{V_{\mathrm{oD}}}{V_{\mathrm{iC}}}=\frac{G_{\mathrm{DD}}^{2}}{\left(1+G_{\mathrm{DD}} / A_{2}\right) \cdot A_{1} \cdot\left(G_{\mathrm{DD}}-1\right)},
$$


y el CMRR resulta:

$$
C M R R=\frac{G_{\mathrm{DD}}}{G_{\mathrm{DC}}}=\frac{\left(1+G_{\mathrm{DD}} / A_{2}\right) \cdot A_{1} \cdot\left(G_{\mathrm{DD}}-1\right)}{G_{\mathrm{DD}}} .
$$

Como $G_{\mathrm{DD}} \gg 1$, puede escribirse:

$$
C M R R \cong\left(1+G_{\mathrm{DD}} / A_{2}\right) \cdot A_{1}=A_{1}+G_{\mathrm{DD}} \cdot A_{1} / A_{2} .
$$

Considerando además que $A_{1} \approx A_{2}$ y que $A_{1} » G_{\mathrm{DD}}$, el CMRR puede finalmente aproximarse por:

$$
C M R R \cong A_{1}
$$

Se observa aquí que el CMRR está dado aproximadamente por la ganancia a lazo abierto del amplificador AO1, que para bajas frecuencias puede ser de unos $110 \mathrm{~dB}$. Si se utilizan A.O compensados, la ganancia $A_{1}(\mathrm{~s})$ decae a partir de una frecuencia muy baja, de solo algunos $\mathrm{Hz}$, por lo que esta estructura resulta útil exclusivamente para aplicaciones de baja frecuencia. Este efecto también es notable cuando se utilizan A.O. de muy bajo consumo, los cuales suelen presentar bajas ganancias de lazo abierto.

Es importante recordar que para llegar al CMRR dado por (A9.18) se supuso un apareamiento perfecto entre los resistores y entre los CMRR de los A.O. En realidad, todos estos efectos contribuirán conjuntamente a degradar el CMRR, que nunca podrá ser mayor que ninguno de los valores dados por (A9.9) y (A9.18).

\section{Tensión Equivalente de Ruido a la entrada}

El circuito equivalente del I.A. de dos A.O., teniendo en cuenta los generadores de ruido asociados a los A.O y a los resistores, se muestra en la Fig.A9.3.

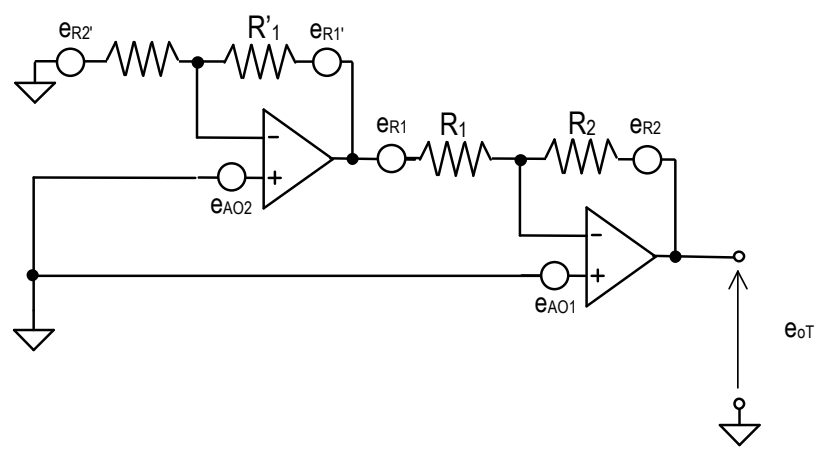

Figura A9.3. Circuito Equivalente para el cálculo del ruido total.

Resolviendo este circuito, se encuentra que la tensión cuadrática media de ruido en su salida está dada por:

$$
e_{\mathrm{oT}}^{2}=\left(e_{\mathrm{AO} 1}^{2}+e_{\mathrm{AO} 2}^{2}\right) \cdot G_{\mathrm{DD}}^{2}+\left(e_{\mathrm{R} 1}^{2}+e_{\mathrm{R} 1^{\prime}}^{2}\right) \cdot G_{\mathrm{DD}}^{2}+e_{\mathrm{R} 2}^{2}+e_{\mathrm{R} 2^{\prime}}^{2} .
$$


Si los resistores son de bajo exceso de ruido, se verifica:

$$
e_{\mathrm{R} 1}^{2}=4 \mathrm{k} T B \cdot R_{1} \quad ; \quad e_{\mathrm{R} 2}^{2}=4 \mathrm{k} T B \cdot R_{2} .
$$

Considerando una ganancia importante, tal que permita aproximar $G_{\mathrm{DD}} \cong R_{2} / R_{1}$, es posible expresar el ruido de $R_{2}$ en función del correspondiente a $R_{1}$ como:

$$
e_{\mathrm{R} 2}^{2}=4 \mathrm{k} T B \cdot R_{2}=G_{\mathrm{DD}} \cdot e_{\mathrm{R} 1}^{2}
$$

Suponiendo además $R_{1} \cong R_{1}{ }^{\prime}$ y $R_{2} \cong R_{2}{ }^{\prime}$, la tensión de ruido total a la salida resulta:

$$
e_{\mathrm{oT}}^{2}=\left(e_{\mathrm{AO} 1}^{2}+e_{\mathrm{AO} 2}^{2}\right) \cdot G_{\mathrm{DD}}^{2}+2 e_{\mathrm{R} 1}^{2} \cdot G_{\mathrm{DD}}^{2}+2 \cdot e_{\mathrm{R} 2}^{2} \cdot G_{\mathrm{DD}},
$$

y el ruido total referido a la entrada puede aproximarse por:

$$
e_{\mathrm{iT}}^{2} \cong e_{\mathrm{AO} 1}^{2}+e_{\mathrm{AO} 2}^{2}+2 e_{\mathrm{R} 1}^{2}
$$

Si el valor de $R_{1}$ no es demasiado elevado, el ruido total en (A9.23) estará dominado por los A.O. y queda dado por:

$$
e_{\mathrm{iT}}^{2} \cong 2 e_{\mathrm{AO}}^{2} \quad,
$$

siendo $e_{\mathrm{AO}}$ el ruido propio correspondiente a un A.O.

\section{Amplificador de Instrumentación de 2 A.O. con ajuste de ganancia.}

Este circuito, que fue presentado en el CAPíTulo 5 y que se muestra en la Fig.A9.4, permite ajustar la ganancia del amplificador de instrumentación modificando un solo elemento: el resistor de ganancia $R_{\mathrm{G}}$.

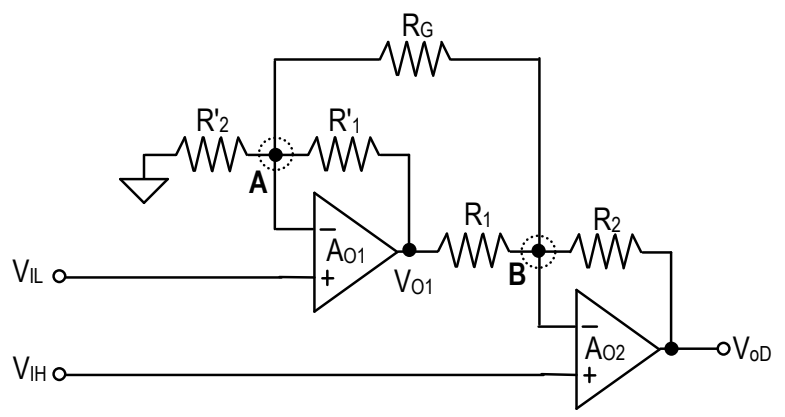

Figura A9.4. Amplificador de Instrumentación de dos A.O. con ajuste de ganancia. 


\subsection{Rechazo de Modo Común.}

El circuito equivalente considerando amplificadores con CMRR finito resulta:

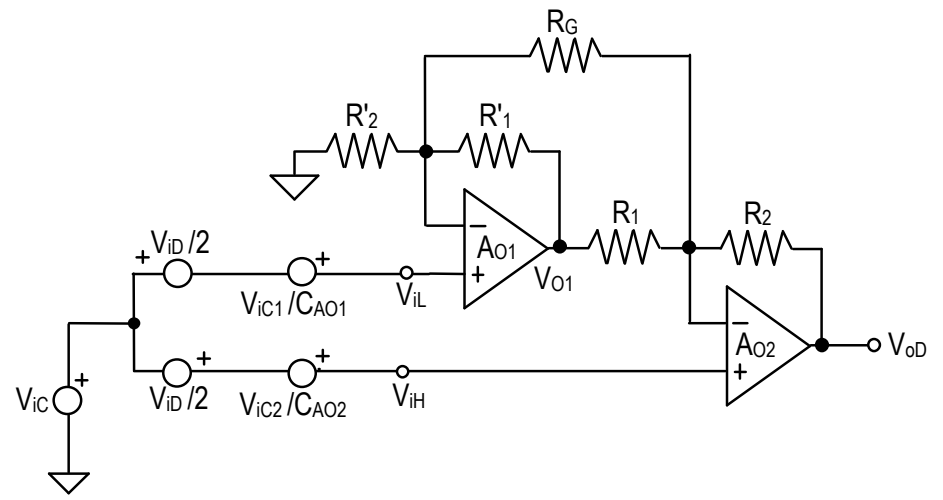

Figura A9.5. Circuito Equivalente que contempla el CMRR propio de los A.O.

La tensión de salida $V_{\mathrm{oD}}$, está dada por

$$
V_{\mathrm{oD}}=\left(V_{\mathrm{iH}}-V_{\mathrm{iL}}\right) \cdot\left\{\frac{R_{2}}{R_{1}}+\frac{R_{1}^{\prime} \cdot R_{2}}{R_{1} \cdot R_{\mathrm{G}}}+\frac{R_{2}}{R_{\mathrm{G}}}\right\}+V_{\mathrm{iH}}-V_{\mathrm{iL}} \cdot \frac{R_{1}^{\prime} \cdot R_{2}}{R_{1} \cdot R_{2}^{\prime}},
$$

donde se consideró como $V_{\mathrm{iH}}, V_{\mathrm{iL}}$ a las tensiones de entrada del I.A formado por los A.O. ideales. Estas tensiones están dadas por:

$$
\begin{aligned}
& V_{\mathrm{iH}}=V_{\mathrm{iC}}+V_{\mathrm{iD}} / 2+V_{\mathrm{iC} 2} \cdot C_{\mathrm{AO} 2}^{-1} \\
& V_{\mathrm{iL}}=V_{\mathrm{iC}}-V_{\mathrm{iD}} / 2+V_{\mathrm{iCl}} \cdot C_{\mathrm{AO} 1}^{-1}
\end{aligned}
$$

Como las tensiones de entrada de modo común de ambos A.O. son aproximadamente iguales a la tensión de modo común de entrada al I.A, esto es $V_{\mathrm{iCl} 1} \cong V_{\mathrm{iC} 2} \cong V_{\mathrm{iC}}$, las ecuaciones en (A9.26) pueden rescribirse como:

$$
\begin{aligned}
& V_{\mathrm{iH}}=V_{\mathrm{iD}} / 2+V_{\mathrm{iC}} \cdot\left(1+C_{\mathrm{AO} 2}^{-1}\right) \\
& V_{\mathrm{iL}}=-V_{\mathrm{iD}} / 2+V_{\mathrm{iC}} \cdot\left(1+C_{\mathrm{AO} 1}^{-1}\right)
\end{aligned}
$$

Reemplazando estas expresiones en (A9.25), resulta:

$$
\begin{aligned}
& V_{\mathrm{oD}}=V_{\mathrm{iD}}\left\{\frac{R_{2}}{R_{1}}+\frac{R_{2}}{R_{\mathrm{G}}} \cdot\left(1+\frac{R_{1}^{\prime}}{R_{1}}\right)+\frac{1}{2} \cdot\left(1+\frac{R_{1}^{\prime} \cdot R_{2}}{R_{1} \cdot R_{2}^{\prime}}\right)\right\}+ \\
& +V_{\mathrm{iC}}\left\{\left(C_{\mathrm{AO2} 2}^{-1}-C_{\mathrm{AO} 2}^{-1}\right) \cdot\left[\frac{R_{2}}{R_{1}}+\frac{R_{2}}{R_{\mathrm{G}}} \cdot\left(1+\frac{R_{1}^{\prime}}{R_{1}}\right)\right]+1+C_{\mathrm{AO2} 2}^{-1}-\frac{R_{1}^{\prime} \cdot R_{2}}{R_{1} \cdot R_{2}^{\prime}} \cdot\left(1-C_{\mathrm{AO} 1}^{-1}\right)\right\}
\end{aligned}
$$

Considerando ganancias medianamente altas y desbalances razonables, la ecuación (A9.28) puede aproximarse como: 


$$
V_{\mathrm{oD}} \cong V_{\mathrm{iD}} \cdot G_{\mathrm{DD}}+V_{\mathrm{iC}} \cdot\left[\left(C_{\mathrm{AO} 2}^{-1}-C_{\mathrm{AO} 2}^{-1}\right) \cdot G_{\mathrm{DD}}+\delta\right],
$$

siendo,

$$
G_{\mathrm{DD}} \cong 1+\frac{R_{2}}{R_{1}}+2 \cdot \frac{R_{2}}{R_{\mathrm{G}}} \quad ; \quad \delta=1-\frac{R_{1}^{\prime} \cdot R_{2}}{R_{1} \cdot R_{2}^{\prime}}
$$

A partir de (A9.29), se obtiene $G_{\mathrm{DC}}$, que está dada por:

$$
G_{\mathrm{DC}} \cong\left(C_{\mathrm{AO} 2}^{-1}-C_{\mathrm{AO} 2}^{-1}\right) \cdot G_{\mathrm{DD}}+\delta
$$

y el rechazo de modo común resulta:

$$
\frac{1}{C M R R}=\left(\frac{1}{C_{\mathrm{AO} 2}}-\frac{1}{C_{\mathrm{AO} 1}}\right)+\frac{\delta}{G_{\mathrm{DD}}}
$$

El parámetro $\delta$ puede expresarse en función de la tolerancia $t$ asociada a los resistores considerando el peor caso como:

$$
\delta_{\mathrm{MAX}} \cong 4 \cdot t
$$

Reemplazando (A9.33) en (A9.32), se obtiene finalmente:

$$
\frac{1}{C M R R}=\left(\frac{1}{C_{\mathrm{AO} 2}}-\frac{1}{C_{\mathrm{AO} 1}}\right)+\frac{4 \cdot t}{G_{\mathrm{DD}}}
$$

donde se observa que el CMRR es el mismo que para el caso del amplificador de dos A.O. $\sin R_{\mathrm{G}}$. La inclusión de este resistor no modifica el CMRR del amplificador de instrumentación.

\subsection{Tensión Eficaz de Ruido}

Incorporando al circuito ideal de la Fig.A9.4, los generadores de ruido correspondientes a los distintos elementos, resulta el circuito de la Fig.A9.6.

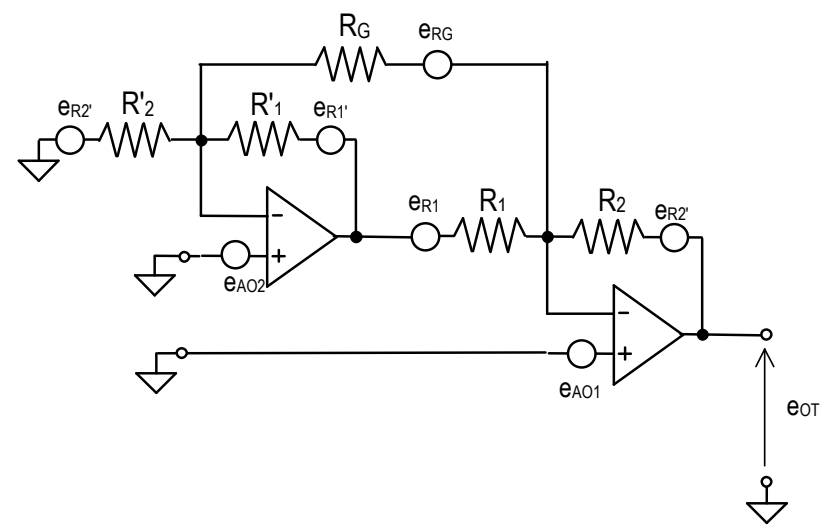

Figura A9.6. Circuito equivalente para el cálculo del ruido total. 
Resolviendo el circuito para cada uno de los generadores presentes en el circuito y aplicando el principio de superposición, se obtiene la siguiente expresión para la tensión de ruido a la salida:

$$
\begin{aligned}
e_{o T}= & e_{R G} \cdot\left(\frac{R_{1}^{\prime} \cdot R_{2}}{R_{G} \cdot R_{1}}+\frac{R_{2}}{R_{G}}\right)+e_{R 1} \cdot \frac{R_{2}}{R_{1}}+e_{R^{\prime} 1} \cdot \frac{R_{2}}{R_{1}}+e_{R 2}+ \\
& +e_{R^{\prime} 2} \cdot \frac{R_{1}^{\prime} \cdot R_{2}}{R_{2}^{\prime} \cdot R_{1}}+\left(e_{A O 1}+e_{A O 2}\right) \cdot G_{D D}
\end{aligned}
$$

Para arribar a esta expresión se tuvo en cuenta que, en el calculo de los efectos de $e_{\mathrm{R} 1}$, $e_{\mathrm{R}^{\prime} 1}, e_{\mathrm{R} 2} \mathrm{y} e_{\mathrm{R}}$ '2, ambos extremos de $R_{\mathrm{G}}$ se encuentran a potencial cero. Entonces la corriente sobre $R_{\mathrm{G}}$ resulta nula y el resistor no interviene en el circuito.

Considerando desbalances razonables y teniendo en cuenta que se trata de una composición de tensiones RMS, resulta:

$$
e_{\mathrm{oT}}=e_{\mathrm{RG}}^{2} \cdot\left(2 \cdot \frac{R_{2}}{R_{\mathrm{G}}}\right)^{2}+2 \cdot e_{\mathrm{R} 1}^{2} \cdot\left(\frac{R_{2}}{R_{1}}\right)^{2}+2 \cdot e_{\mathrm{R} 2}^{2}+2 \cdot e_{\mathrm{AO}}^{2} \cdot G_{\mathrm{DD}}^{2}
$$

Cabe aquí una observación. En (A9.30) se distinguen dos mecanismos que determinan la ganancia total: $R_{2} / R_{1}$ y $2 \cdot R_{2} / R_{\mathrm{G}}$. En (A9.36) se observa que el primero amplifica el ruido en $R_{\mathrm{G}}$ mientras que el segundo tiene efecto sobre el ruido de $R_{2}$.

Si $R_{\mathrm{G}} » R_{2}\left(G_{\mathrm{DD}} \cong R_{2} / R_{1}\right)$, el ruido total referido a la entrada coincide con el calculado para el I.A de dos A.O. Entonces,

$$
\text { si } R_{\mathrm{G}} \gg R_{2}: \quad e_{\mathrm{iT}}^{2}=2 \cdot e_{\mathrm{R} 1}^{2}+2 \cdot e_{\mathrm{AO}}^{2} .
$$

Si por el contrario, $R_{\mathrm{G}}$ « $R_{2}$, 1a ganancia está determinada principalmente por la relación $R_{2} / R_{\mathrm{G}}\left(G_{\mathrm{DD}} \cong 2 \cdot R_{2} / R_{\mathrm{G}}\right)$ y el ruido total referido a la entrada resulta:

$$
\text { si } R_{\mathrm{G}} \ll R_{2}: \quad e_{\mathrm{iT}}^{2}=e_{\mathrm{RG}}^{2}+2 \cdot e_{\mathrm{AO}}^{2}
$$

De todos modos, a menos que existan restricciones importantes de consumo, los resistores se elegirán de valores suficientemente bajos para que su ruido sea despreciable frente al de los A.O. Este último es normalmente el dominante y el ruido total referido a la entrada queda dado por:

$$
e_{\mathrm{iT}}^{2} \cong 2 \cdot e_{\mathrm{AO}}^{2}
$$


Apéndice 10.

\section{Caracterización de la Interferencia producida por tubos fluorescentes}

\section{Introducción}

Los tubos fluorescentes utilizados en iluminación constituyen una fuente de interferencia electromagnética (EMI) adicional para la instrumentación biomédica. Esta interferencia, ingresa al sistema de medida por los mismos mecanismos que la tensión de línea y sus componentes espectrales se extienden hasta frecuencias algo más elevadas.

Algunas publicaciones reportan que su energía se ubica espectralmente en el rango de $1-10 \mathrm{KHz}$ (WINTER \& WEBSTER, 1973), mientras otras la asocian a la banda de 50 a $500 \mathrm{~Hz}$ (SILVA, 1994). Dada esta discrepancia se decidió obtener medidas propias.

\section{Descripción del ensayo.}

El esquema de la disposición utilizada se muestra en la Fig.A11.1. Se conectó un osciloscopio de forma tal de medir la tensión del paciente respecto de tierra y como fuente de interferencia se utilizó un tubo fluorescente de $20 \mathrm{~W}$ que se ubicó a unos 20 $\mathrm{cm}$ del paciente.

La tensión medida corresponde a la tensión definida como $V_{\mathrm{P} 0}$ en el CAPÍTULO 2. La misma se repartirá entre las tensiones de modo común $V_{\mathrm{CM}}$ y de modo aislante $V_{\mathrm{ISO}}$, las cuales interferirán, de acuerdo a distintos mecanismos, con la señal biológica.

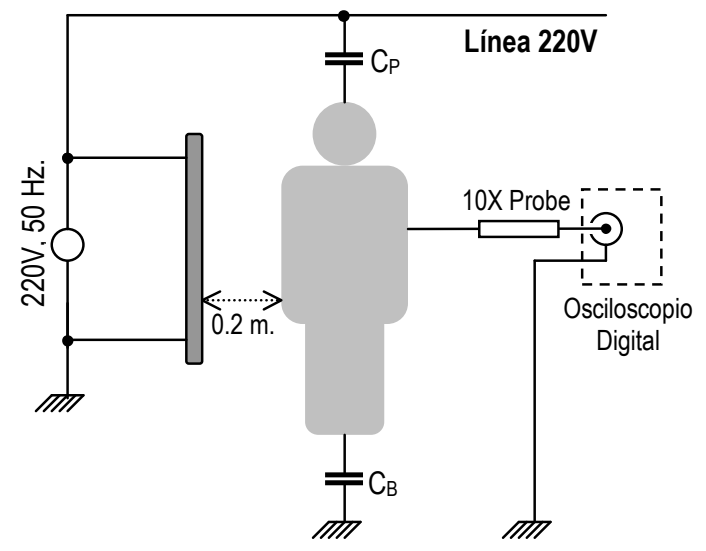

Figura A11.1. Esquema utilizado en las experiencias. Las capacidades $C_{P}$ y $C_{B}$ representan las capacidades propias del paciente respecto de línea y tierra respectivamente. 
En primer lugar, con toda la iluminación del lugar apagada y el tubo fluorescente interferente desconectado (tanto el conductor de fase como el neutro), se registró la tensión debido al acoplamiento de la red de potencia.

Como segundo paso, se encendió la luz fluorescente y se adquirió la nueva tensión resultante entre el paciente y tierra. Los registros obtenidos se muestran en la Fig.A11.2.
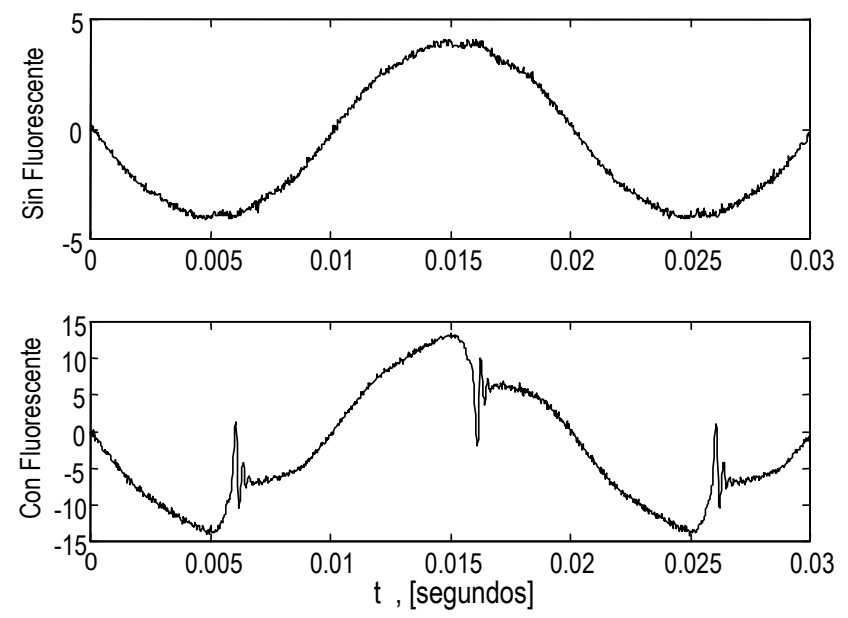

Figura A11.2. Tensión Paciente-Tierra con y sin interferencia de tubos fluorescentes.

Tomando la diferencia entre las dos medidas realizadas, se puede determinar el aporte del equipo fluorescente y su conexionado a la tensión paciente-tierra. El mismo se muestra en la Fig.A11.3.

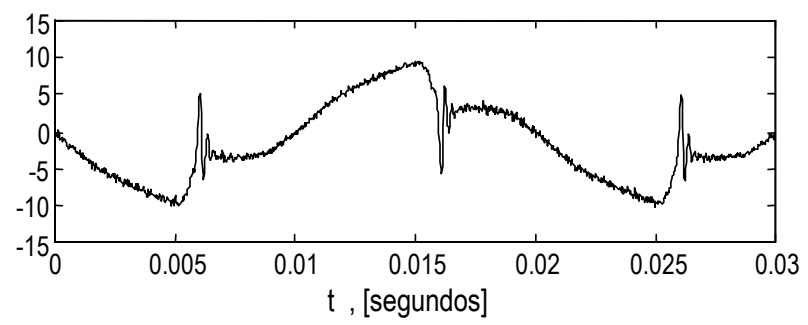

Figura A11.3. Aporte del tubo fluorescente y su cableado a la tensión pacientetierra.

El contenido armónico de esta señal se presenta en la Fig.A11.4. Se observa que son importantes los armónicos impares mientras que los pares son de reducida energía. No se observan componentes importantes más allá del armónico $15(750 \mathrm{~Hz})$, lo cual concuerda con lo expuesto en (SILVA, 1994). Sin embargo, en la Fig.A11.3 se observa que existe una señal de alta frecuencia de amplitud considerable. La misma no es evidente en el análisis de contenido armónico pues su energía es reducida. 


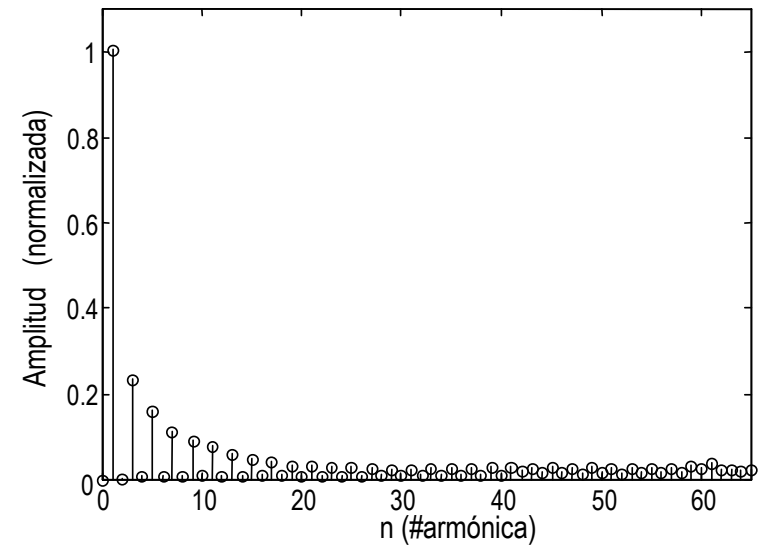

Figura A11.4. Contenido armónico de la tensión paciente-tierra

Para poner en evidencia la existencia de esta interferencia de alta frecuencia, la señal de EMI fue descompuesta en tres componentes: una sinusoidal de $50 \mathrm{~Hz}$, una segunda componente conteniendo armónicas de baja frecuencia y una última que abarca el espectro restante. En la Fig.A11.5 se muestran estas señales, que compuestas reconstruyen la original.

La segunda componente contiene armónicas de la frecuencia de red de hasta algunos cientos de Hertz., lo cual concuerda con lo reportado en (SILVA, 1994).

La última de las componentes descriptas, que abarca las altas frecuencias, posee su energía fundamentalmente concentrada en el rango comprendido entre 1-4 KHz lo cual concuerda con lo expuesto en (WINTER \& WEBSTER, 1973). Se observa que si bien su energía es reducida, su amplitud es importante pudiendo llegar a comprometer el rango dinámico de los amplificadores. Además, en presencia de no-linealidades, por ejemplo en la etapa de entrada o en las impedancias electródicas, estas componentes pueden también producir señales no deseadas de baja frecuencia (DE JAGER, 1996). 

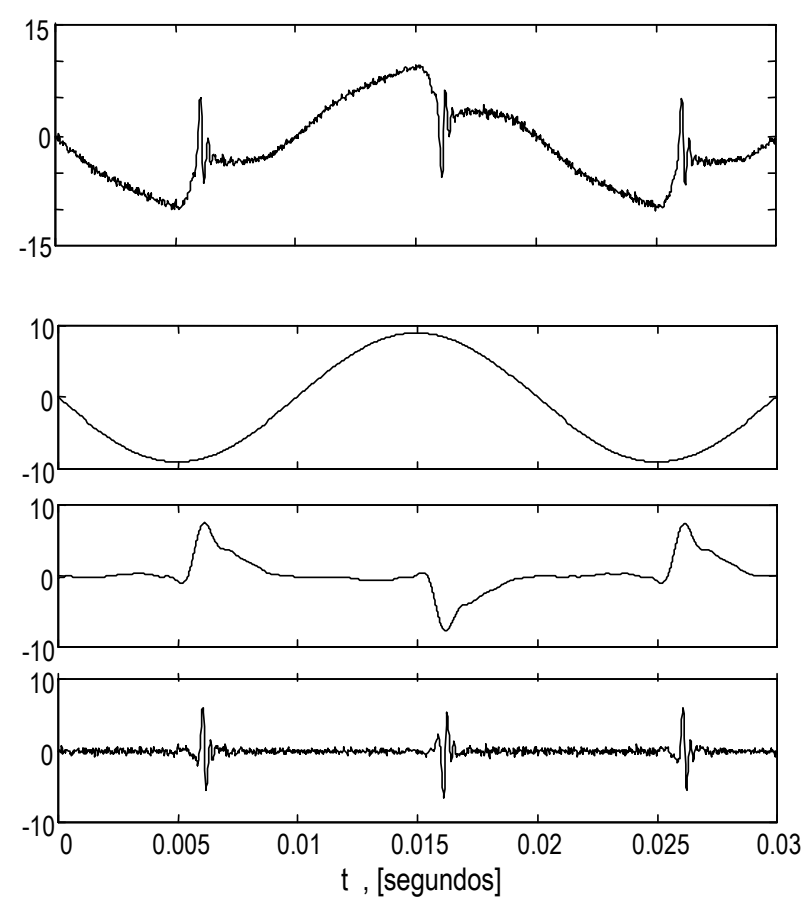

Figura A11.5. Descomposición de la tension de modo común en tres componentes.

\section{Conclusiones}

La interferencia producida por los tubos fluorescentes presenta componentes de energía importante para frecuencias de hasta unos $750 \mathrm{~Hz}$, pero contiene además componentes de alta frecuencia, fundamentalmente en el rango entre 1- 4kHz. Estas últimas, si bien no tienen una energía importante, pueden componer un valor pico elevado, y debido a no-linealidades en la etapa de entrada o en las interfases electrodopiel, pueden también producir componentes no deseadas en baja frecuencia. 


\section{Apéndice 11.}

\section{Sensibilidad de las raíces de un polinomio a variaciones en sus coeficientes.}

\section{Análisis de sensibilidad.}

Dado un polinomio de la forma:

$$
P(s)=a_{0} s^{n}+a_{1} s^{n-1}+a_{2} s^{n-2}+\cdots+a_{n} \quad,
$$

con raíces $\left(\lambda_{1}, \lambda_{2}, \ldots, \lambda_{\mathrm{n}}\right)$, que puede escribirse alternativamente como:

$$
P(s)=a_{0}\left(s-\lambda_{1}\right)\left(s-\lambda_{2}\right) \cdots\left(s-\lambda_{n}\right) \quad .
$$

La variación en la ubicación de la raíz $i$-ésima $\lambda_{\mathrm{i}}$, debido a un desplazamiento diferencial en el coeficiente $j$-ésimo $a_{j}$, está dado por (BOGNER, 1975):

$$
\Delta \lambda_{i}=\frac{\lambda_{i}^{n-j}}{a_{0} \prod_{k \neq i}\left(\lambda_{k}-\lambda_{i}\right)} \cdot \Delta a_{j}
$$

En el caso particular de un polinomio de segundo grado, la variación en las raíces, debidas a incrementos en los coeficientes, resulta:

$$
\begin{aligned}
& \Delta \lambda_{1}=\frac{\lambda_{1}^{2}}{a_{0}\left(\lambda_{2}-\lambda_{1}\right)} \cdot \Delta a_{0}+\frac{\lambda_{1}}{a_{0}\left(\lambda_{2}-\lambda_{1}\right)} \cdot \Delta a_{1}+\frac{1}{a_{0}\left(\lambda_{2}-\lambda_{1}\right)} \cdot \Delta a_{2} \\
& \Delta \lambda_{2}=\frac{\lambda_{2}^{2}}{a_{0}\left(\lambda_{1}-\lambda_{2}\right)} \cdot \Delta a_{0}+\frac{\lambda_{2}}{a_{0}\left(\lambda_{1}-\lambda_{2}\right)} \cdot \Delta a_{1}+\frac{1}{a_{0}\left(\lambda_{1}-\lambda_{2}\right)} \cdot \Delta a_{2}
\end{aligned}
$$

o en una forma algo más compacta:

$$
\begin{aligned}
& \Delta \lambda_{1}=\frac{1}{a_{0}\left(\lambda_{2}-\lambda_{1}\right)}\left[\lambda_{1}^{2} \Delta a_{0}+\lambda_{1} \Delta a_{1}+\Delta a_{2}\right] \\
& \Delta \lambda_{2}=\frac{-1}{a_{0}\left(\lambda_{2}-\lambda_{1}\right)}\left[\lambda_{2}^{2} \Delta a_{0}+\lambda_{2} \Delta a_{1}+\Delta a_{2}\right]
\end{aligned}
$$


Apéndice 11 - Sensibilidad de las raíces de un polinomio variaciones en sus coeficientes 


\section{Apéndice 12.}

\section{Tensión de Ruido debida a los Resistores de polarización.}

\section{Calculo de la Tensión de Ruido.}

La red de acoplamiento pasiva, propuesta en el CAPítulo 6, contiene resistores de valor elevado cuyo ruido propio puede ser importante. En este apéndice se analiza la contribución de estas fuentes de ruido a la señal disponible en la entrada del A.B., es decir a la salida de la red.

Para analizar esta contribución resulta suficiente el circuito equivalente para modo diferencial de la Fig.A13.1. Este circuito supone una señal de entrada nula y modela la impedancia de electrodo como un resistor $R_{\mathrm{E}}$.

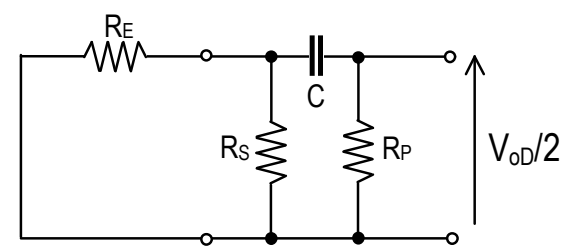

Figura A13.1. Circuito Equivalente de la Red de Acoplamiento para modo diferencial.

Cada uno de los resistores de este circuito tienen asociada una tensión de ruido; siendo significativos los correspondientes a $R_{\mathrm{S}}$ y $R_{\mathrm{P}}$, dado que estos últimos resistores normalmente son de valor mucho más elevado que $R_{\mathrm{E}}$. Como $R_{\mathrm{S}}$ queda en paralelo con $R_{\mathrm{E}}$ su contribución no será importante y el circuito equivalente para análisis de ruido se reduce al de la Fig.A13.2.

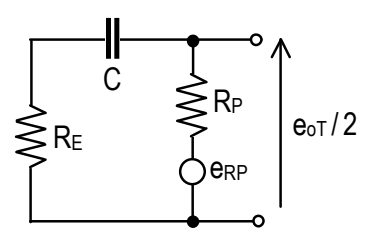

Figura A13.2. Circuito Equivalente para análisis de ruido que contempla el ruido propio de $R_{\mathrm{P}}$.

Considerando que el resistor $R_{\mathrm{P}}$ tiene una densidad espectral de ruido $\eta_{\mathrm{RP}}$ constante, la densidad espectral del ruido a la salida $\eta_{\mathrm{eo}}$ está dada por: 


$$
\eta_{e o}=\eta_{R_{\mathrm{P}}}\left|\frac{1+\mathrm{s} \cdot R_{\mathrm{E}} C}{1+\mathrm{s} \cdot\left(R_{\mathrm{P}}+R_{\mathrm{E}}\right) C}\right|^{2}
$$

Teniendo en cuenta que $R_{\mathrm{P}} \gg R_{\mathrm{E}}$, y llamando

$$
\tau_{\mathrm{P}}=R_{\mathrm{P}} C \quad ; \quad \tau_{\mathrm{E}}=R_{\mathrm{E}} C \quad,
$$

(A13.1) puede aproximarse por:

$$
\eta_{e o}=\eta_{R_{\mathrm{P}}}\left|\frac{1+s \cdot \tau_{\mathrm{E}}}{1+s \cdot \tau_{\mathrm{P}}}\right|^{2}
$$

La gráfica de la densidad espectral a la salida se muestra en la Fig.A13.3.

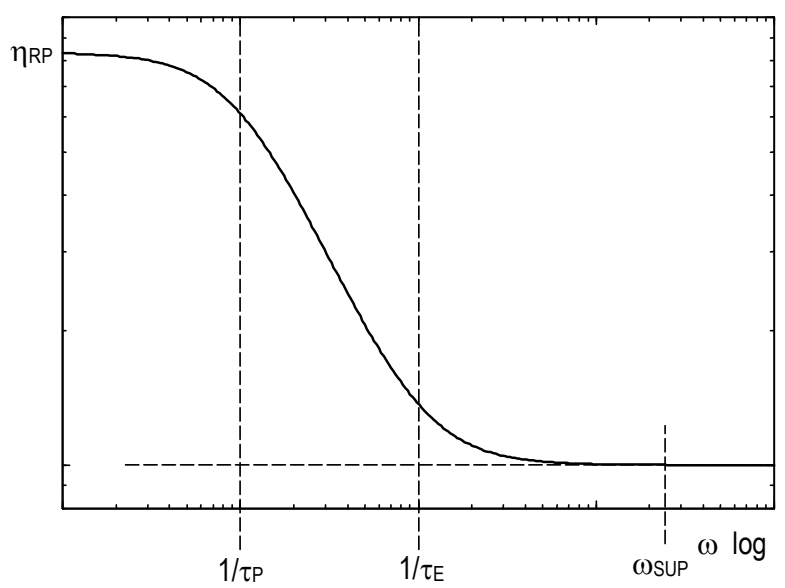

Figura A13.3. Densidad Espectral de ruido a la salida de la red de acoplamiento.

Para determinar el ruido total, es necesario integrar bajo esta curva en el rango de frecuencias de interés, es decir desde $1 / \tau_{\mathrm{P}}$ hasta $\omega_{\text {sup. }}$. En este intervalo se distinguen dos zonas: en la primera la densidad espectral decrece con el cuadrado de $\omega$ según:

$$
\eta_{e o} \cong \eta_{R_{\mathrm{P}}} \frac{1}{\left(\omega R_{\mathrm{P}} C\right)^{2}}
$$

y en la segunda, la densidad espectral se vuelve constante:

$$
\eta_{e o} \cong \eta_{R_{\mathrm{P}}}\left(\frac{R_{\mathrm{E}}}{R_{\mathrm{P}}}\right)^{2}
$$

Teniendo en cuenta las aproximaciones correspondientes a cada tramo, el ruido total queda dado por:

$$
e_{o \mathrm{~T}}=\int_{1 / \tau_{\mathrm{P}}}^{\omega_{\mathrm{SUP}}} \eta_{R_{\mathrm{P}}} d \omega \cong \eta_{R_{\mathrm{P}}} \cdot\left\{\int_{1 / \tau_{\mathrm{P}}}^{1 / \tau_{\mathrm{E}}} \frac{1}{\left(\tau_{\mathrm{P}} \omega\right)^{2}} d \omega+\int_{1 / \tau_{\mathrm{E}}}^{\omega_{\mathrm{SUP}}}\left(\frac{R_{\mathrm{E}}}{R_{\mathrm{P}}}\right)^{2} d \omega\right\}
$$


Resolviendo esta integral resulta:

$$
e_{o \mathrm{~T}}^{2} \cong \eta_{R_{\mathrm{P}}} \frac{\left(\tau_{\mathrm{P}}-\tau_{\mathrm{E}}\right)}{\tau_{\mathrm{P}}^{2}}+\eta_{R_{\mathrm{P}}}\left(\frac{R_{\mathrm{E}}}{R_{\mathrm{P}}}\right)^{2}\left(\omega_{\mathrm{SUP}}-1 / \tau_{\mathrm{E}}\right)
$$

y recordando que $\eta_{R_{\mathrm{P}}}=4 \mathrm{k} T R_{\mathrm{P}}$, la ecuación (A13.7) puede rescribirse como:

$$
e_{o \mathrm{~T}} \cong \frac{4 k T}{C}+4 \mathrm{k} T \frac{R_{\mathrm{E}}^{2}}{R_{\mathrm{P}}}\left(\omega_{\mathrm{SUP}}-1 / \tau_{\mathrm{E}}\right)
$$

El segundo término corresponde al ruido de un resistor equivalente $R_{\mathrm{E}}{ }^{2} / R_{\mathrm{P}}$ y usualmente es despreciable. Por ejemplo si $R_{\mathrm{P}}=5 \mathrm{M} \Omega$ y $R_{\mathrm{E}}=100 \mathrm{k} \Omega$, este término corresponderá al ruido de un resistor de $2 \mathrm{k} \Omega$ cuya tensión de ruido equivalente $(\approx 5 \mathrm{nV} / \sqrt{ } \mathrm{Hz})$ está por debajo del ruido propio de un buen I.A. Despreciando este término, el ruido total resulta:

$$
e_{o T}^{2} \cong \frac{4 k T}{C}
$$

Este es un resultado interesante. Indica que el ruido total no depende de $R_{\mathrm{P}}$ sino solamente del valor del capacitor $C$. Es conveniente utilizar un capacitor de acoplamiento $C$ del mayor valor posible.

\section{Un ejemplo.}

Considerando $R_{\mathrm{P}}=5 \mathrm{M} \Omega, C=1 \mu \mathrm{F}$, que definen una frecuencia de corte inferior $f_{\text {inf }}=0.03 \mathrm{~Hz}$, el ruido total introducido por los resistores $R_{\mathrm{P}}$ resulta:

$$
e_{o T} \cong 0.12 \mu \mathrm{V}_{\mathrm{RMS}}
$$

Esta contribución no es significativa. Se puede decir que la impedancia de los electrodos "cortocircuita" la tensión de ruido de la resistencia de polarización $R_{\mathrm{P}}$.

Para este ejemplo, considerando $R_{\mathrm{E}}=100 \mathrm{k} \Omega$ y una frecuencia de corte superior de $100 \mathrm{~Hz}$, el aporte del segundo término en (A13.7) sería de solo $50 \mathrm{nV}_{\mathrm{RMS}}$, es decir que la aproximación utilizada para arribar a (A13.9) es razonable. 